\title{
Die Steuerung des Technologietransfers in der Volksrepublik China
}

Das Chinesische Recht des Technologietransfers

und Perspektiven über die weitere Entwicklung

\author{
Dissertation \\ Zur Erlangung des Doktorgrades \\ der Juristischen Fakultät \\ der Georg-August-Universität zu Göttingen \\ vorgelegt \\ von \\ WANG, Zhen \\ aus Wuhan, China \\ Göttingen 2008
}


D7

Der erster Gutachter: Prof. Dr. Peter-Tobias Stoll

Der zweiter Gutachter: Prof. Dr. Hans-Martin Müller-Laube

Der Tag der mündlichen Prüfung: 22. April 2008 


\section{Vorwort}

Die juristische Fakultät der Georg-August-Universität Göttingen hat die vorliegende Arbeit im Wintersemester 2007/2008 als Dissertation angenommen. Das Manuskript befindet sich im März 2007; wichtige neuere Veröffentlichungen konnten noch bis Februar 2008 berücksichtigt werden.

Ohne vielfältige Hilfe und Unterstützung wäre diese Arbeit nicht zustande gekommen. An dieser Stelle möchte ich meinen Dank aussprechen an all diejenigen, die mich während der Bearbeitung dieser Dissertation begleitet und mir Mut und Kraft gegeben haben. Zunächst gilt mein ganz besonderer Dank meinem Doktorvater, Herr Prof. Dr. Peter-Tobias Stoll, der die Anregung zu diesem Thema gab und die Fertigstellung der Arbeit in vielfältiger Weise gefördert hat. Für seine Betreuung während meines ganzen Studiums in Deutschland danke ich ihn sehr herzlich. Herrn Prof. Dr. Hans-Martin Müller-Laube danke ich für die zügige Erstellung des Zweitgutachtens. Nicht zuletzt gilt mein besonderer Dank meinen Eltern, die mich bei der juristischen Ausbildung und der Verfassung der Dissertation finanziell unterstützt haben. Ich danke auch all denen, die mir ihre Freundschaft geschenkt haben und mit derer Hilfe ich Deutschland besser kennen lernen konnte. 


\section{Inhaltsübersicht}

Vorwort...............................................................................

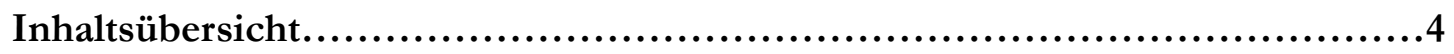

Inhaltsverzeichnis..........................................................6

Abkürzungsverzeichnis...................................................16

Einführung................................................................19

Erster Teil: $\quad$ Technologietransfer......................................22

A. Begriffserklärung...................................................22

B. Gegenstand des Technologietransfers................................23

C. Formen des Technologietransfers.....................................31

D. Vertrag zur Übertragung von Patentrechten und Rechte zur Patentanmeldung...................................................32

E. Patentlizenzvertrag.................................................32

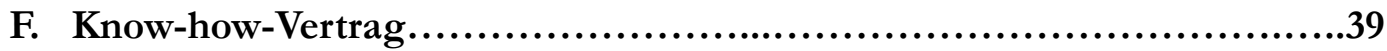

Zweiter Teil: Rahmenbedingungen zum Technologietransfer in China.........42

A. Die Interessenlage beim Technologietransfer und nationale Rechtsordnung der Entwicklungsländer.............................42

B. Die Gesetzgebung zum Technologietransfer in China...................48

Dritter Teil: Der Rechtsschutz des geistigen Eigentums in der VR China....................................................62

A. Grundzüge über den Rechtsschutz des geistigen Eigentums............62

B. Rechtsschutz des geistigen Eigentums in China im Vergleich mit dem 
Immaterialgüterrecht in Deutschland.................................70

C. Der Schutz des Know-How........................................93

D. Rechtsdurchsetzung zum Schutz des geistigen Eigentums......................................................

E. Zusammenfassung...............................................

Vierter Teil: Vertragrechtliche und kartellrechtliche Grundlagen zum Technologietransfer.......................................99

A. Einführung in die Problematik.....................................99

B. Die Rechtsbeziehungen im Vertrag über den TechnologieTransfer..........................................................102

C. Das neue chinesische Kartellgesetz im Vergleich zum EU-Kartellrecht..................................................117

D. Gruppenfreistellungsverordnung für Technologietransfer-

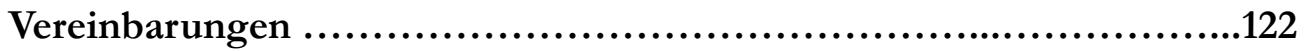

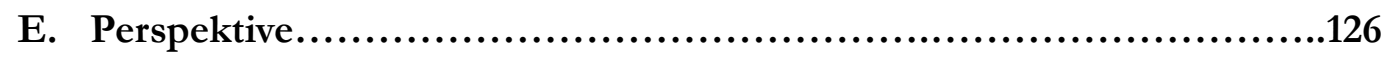

Zusammenfassung......................................................127

Antimonopolgesetz der VR China.........................................131

Literaturverzeichnis...................................................... 


\section{Inhaltsverzeichnis}

Vorwort................................................................................

Inhaltsübersicht...............................................................4

Inhaltsverzeichnis...........................................................6

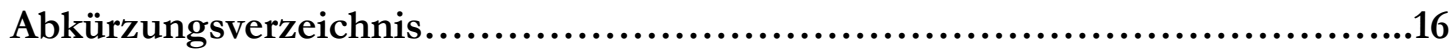

Einführung..............................................................

Erster Teil: Technologietransfer..........................................22

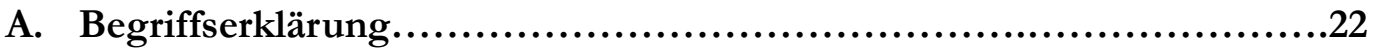

B. Gegenstand des Technologietransfers................................23

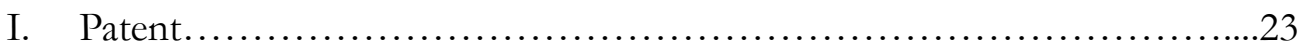

1. Gegenstand des Patentschutzes................................23

2. Patentfähigkeit.................................................24

3. Rolle des Patents beim Technologietransfer..........................24

II. Gebrauchsmuster..............................................24

III. Geschmacksmuster.................................................25

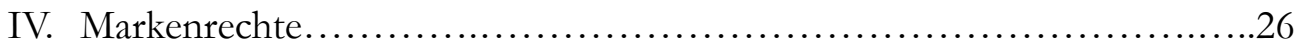

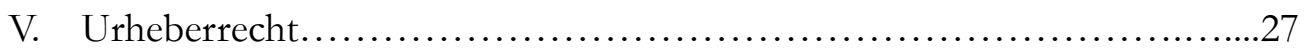

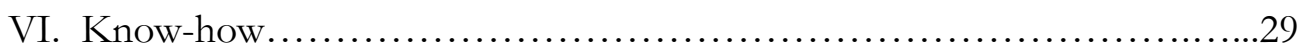

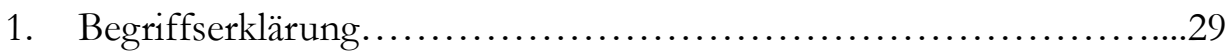

2. Rechtsnatur................................................... 30

C. Formen des Technologietransfers....................................31

D. Vertrag zur Übertragung von Patentrechten und Rechte zur Patentanmeldung......................................................

E. Patentlizenzvertrag................................................32

I. Einführung..................................................... 32 
II. Rechtsnatur des Lizenzvertrages...................................33

III. Die Arten des Lizenzvertrages....................................... 34

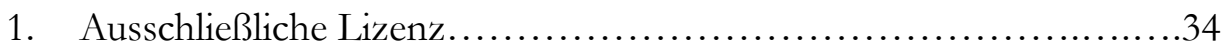

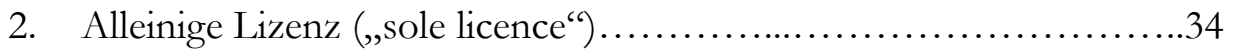

3. Einfache Lizenz............................................. 34

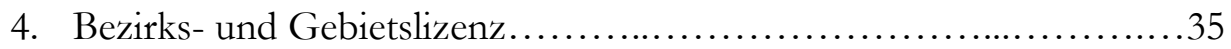

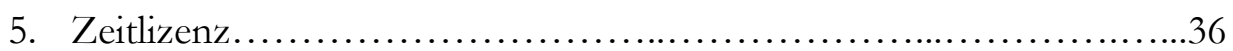

6. Betriebslizenz (Unternehmenslizenz) ................................36

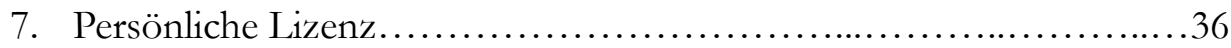

8. Konzernlizenz.................................................. 37

9. Quotenlizenz...................................................

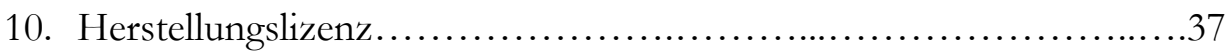

11. Vertriebslizenz................................................

12. Gebrauchslizenz............................................. 38

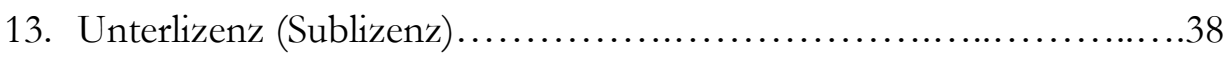

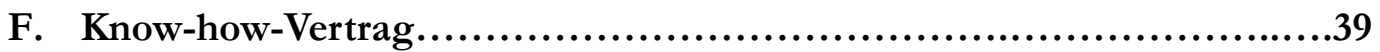

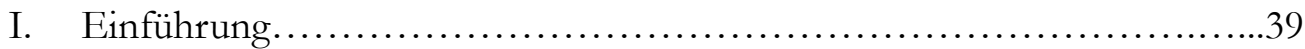

II. Rechtsnatur des Know-how-Vertrages....................................39

III. Arten des Know-how-Vertrages.................................40

1. Einseitiger und zweiseitiger Know-how-Vertrag.....................40

2. Einfache und ausschließliche Know-how-Erteilung..................40

Zweiter Teil: Rahmenbedingungen zum Technologietransfer in China...........42

\section{A. Die Interessenlage beim Technologietransfer und nationale Rechtsordnung der Entwicklungsländer..............................42}

I. Einführung in die Problematik...................................42

II. Interesse des Technologiegebers am Technologietransfer................43

III. Interesse des Technologienehmers am Technologietransfer...............43

IV. Die Rolle des Patentwesens für den Technologietransfer an Entwicklungsländer............................................44

V. Nationale Rechtsordnung der Entwicklungsländer beim Technologietransfer.............................................45

1. Das nationale Entwicklungsrecht................................45

2. Staatliche Kontrolle über Technologietransferverträge..................46

3. Einschränkungen der Patentfähigkeit.............................46

4. Verkürzung der Schutzfrist...................................47

5. Verschärfung der Zwangslizenzvorschriften.........................47

VI. Zusammenfassung............................................48

B. Die Gesetzgebung zum Technologietransfer in China...................48 
I. Einführung ins nationale Recht

1. Zielsetzung und Grundprinzipien des Wirtschafts

-Verwaltungsrechts..........................................48

a. Nichtdiskriminierungsprinzip.............................49

b. Das Prinzip offener Märkte...............................50

c. Transparenzprinzip...................................... 51

2. Zuständigkeit für den Technologietransfer in China...................51

3. Rechtsquellen............................................ 51

II. Regelungen über Technologietransfer im zivilrechtlichen Bereich.............52

1. Die Allgemeinen Grundsätze des Zivilrechts........................52

2. Technologietransfer nach dem Vertragsgesetz der VR China von

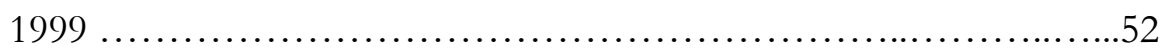

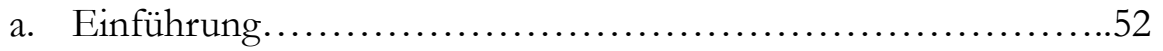

b. Anwendungsbereich des chin. VG...........................53

c. Begriffserklärung..................................... 53

d. Inhalt der Technikverträge................................54

e. Nichtigkeitsgrund.......................................54

f. Verträge über die Entwicklung von Techniken....................55

g. Verträge über technische Beratung und Verträge über technische Dienstleistungen.......................................55

h. Verträge über den Technologietransfer........................55

3. Technologietransfer bei der Gründung der Unternehmen mit ausländischer Kapitalbeteiligung...............................56

III. Wirtschaftsverwaltungsrechtliche Regelungen zum

Technologietransfer.............................................56

1. Das Außenhandelsgesetz der VR China von 2004......................56

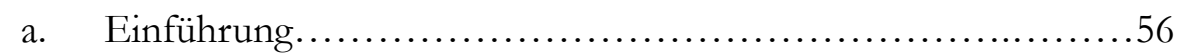

b. Außenhandelsbetreiber.....................................57

c. Der Im- und Export von Waren und Technologie.................58

d. Vorschriften zum Schutz des geistigen Eigentums.................58

2. Die Verwaltungsbestimmungen für den Technologieimport und - Export................................................. 58

a. Einführung...........................................58

b. Anwendungsbereich......................................59

c. Grundsatz des freien Verkehrs der Technologie....................59

d. FIE...................................................... 59

e. Genehmigungsverfahren für die Lizenzerteilung....................60

i. Freie Technologie....................................60

ii. Beschränkte Technologie...........................60

iii. Sonstiges.........................................60

f. Haftung, Geheimhaltung....................................61

g. Verbot wettbewerbswidriger Bestimmungen.....................61

3. Vorschriften zur Lenkung ausländischer Investitionen am 11. Februar 
Dritter Teil: Der Rechtsschutz des geistigen Eigentums in der VR

China.... .62
A. Grundzüge
über
den
Rechtsschutz
des
geistigen
Eigentums

I. Grundzüge des internationalen Rechtsschutzes des geistigen

Eigentums

1. Theorien zum Patentschutz

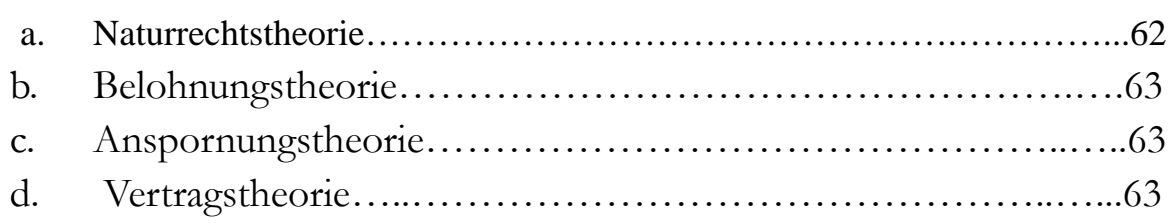

2. Grundprinzipien der gewerblichen Schutzrechte.....................63

a. Numerus clausus.......................................63

b. Territorialitätsprinzip...................................64

c. Erschöpfungsprinzip (Konsumption) .......................64

d. Prioritätsprinzip......................................64

3. Internationale Übereinkommen in Bezug auf das gewerbliche

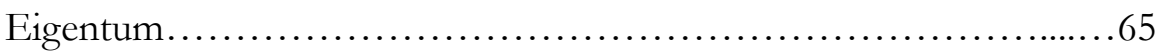

a. Pariser Verbandsübereinkunft...........................65

i. Einführung........................................65

ii. Grundprinzipien der PVÜ............................65

b. Patentzusammenarbeitsvertrag..............................66

c. Haager Musterabkommen...................................66

d. Madrider Markenabkommen ...............................66

e. TRIPS-Übereinkommen...............................67

II. Bedeutung des Patentschutzes für den technischen

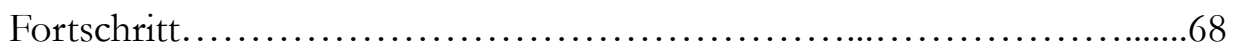

1. Anreiz für Forschung und Entwicklung.............................68

2. Förderung der Anwendung und Verbreitung technischen

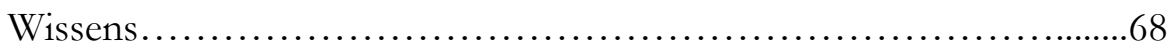

3. Patentschutz als Instrument des internationalen Technologietransfers........................................68

III. Rechtsschutz des geistigen Eigentums und Kartellrecht........................69

1. Das Spannungsverhältnis zwischen Patent und Kartellrecht...........69

2. Der Missbrauchstatbestand............................................69

a. Schutzeffekt als Innovationshemmnis..........................69

b. Schutzeffekt als Diffusionshemmnis.............................70 
c. Lizenzpolitik der Unternehmen.............................70

\section{B. Rechtsschutz des geistigen Eigentums in China im Vergleich mit dem} Immaterialgüterrecht in Deutschland...............................70

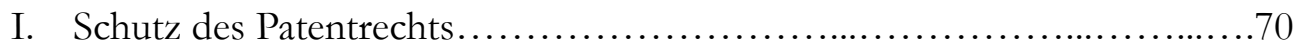

1. Einführung............................................... 70

2. Patentrechtsverletzungen....................................... 72

a. im deutschen Recht.....................................72

i. Die unmittelbaren Benutzungshandlungen...................72

ii. Verwendung von Mitteln zur Erfindungsbenutzung (mittelbare Verletzung) ................................72

iii. Erlaubte Benutzungshandlungen........................73

a). Handlungen im privaten Bereich zu nichtgewerblichen Zwecken.......................................73

b). Handlungen zu Versuchszwecken........................73

c). Einzelzubereitung von Arzneimitteln..................73

d). Einrichtungen an ausländischen Fahrzeugen..............73

iv. Vorbenutzungsrecht..................................73

v. Staatliche Benutzungsordnung..........................73

b. im chinesischen Recht.................................74

i. Anmaßung fremden und nicht bestehenden Patentrechts...........................................74

ii. Patentverletzungen......................................... 74

iii. Gebrauchsmusterverletzungen..........................75

iv. Ausnahmen............................................. 75

a). Art. 63 Abs. 1 Ziff. 1 chin. PatG.........................75

b). Art. 63 Abs. 1 Ziff. 2 chin. PatG........................ 75

c). Art. 63 Abs. 1 Ziff. 3 chin. PatG......................... 75

d). Art. 63 Abs. 1 Ziff. 4 chin. PatG........................76

e). Art. 63 Abs. 2 chin. PatG................................76

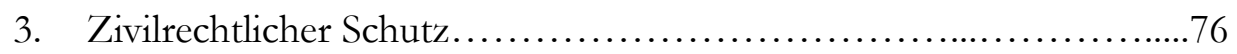

a. im deutschen Recht........................................ 76

i. Unterlassungsanspruch...............................76

ii. Schadensersatzanspruch................................ 76

iii. Beseitigungsanspruch..................................76

iv. Auskunftsanspruch..................................77

v. Umkehr der Beweislast....................................77

b. im chinesischen Recht...................................... 77

i. $\quad$ Ermutigung der Parteien zur Absprache.................... 77

ii. Haftung für Rechtsverletzung............................77

a). Unterlassung........................................ 77

b). Schadensersatz........................................ 78

c). Haftung aus chin. AGZR.............................78 
4. Verwaltungsrechtlicher Schutz...................................78

a. Allgemeines..........................................78

b. Patentverwaltungsbehörde..................................78

c. Behördliche Schutzmaßnahmen................................79

5. Strafrechtlicher Schutz................................................. 79

a. im deutschen Recht.......................................79

b. im chinesischen Recht.....................................79

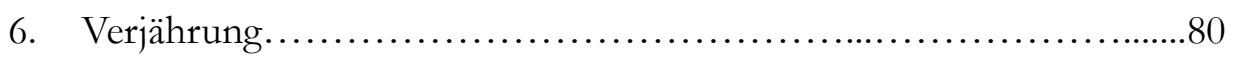

a. im deutschen Recht...................................... 80

b. im chinesischen Recht......................................80

II. Schutz des Markenrechts............................................ 80

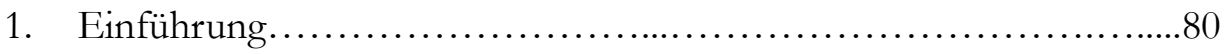

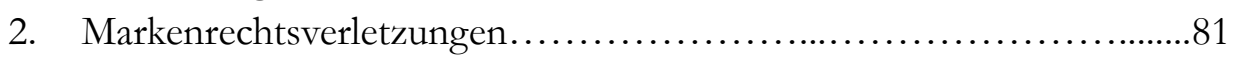

a. im deutschen Recht..................................... 81

i.Identität............................................... 81

ii. Verwechslungsgefahr................................... 81

iii. Schutz bekannter Marken................................82

iv. Ausdrücklich geregelte unzulässige

Benutzungshandlungen..................................82

v. Vorbereitungshandlungen................................ 83

b. im chinesischen Recht.......................................... 83

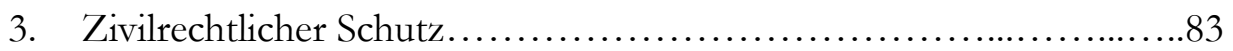

a. im deutschen Recht........................................83

i. Unterlassungsanspruch.............................. 83

ii. Schadensersatzanspruch.............................. 84

iii. Vernichtungsanspruch....................................84

iv. Auskunftsanspruch.......................................84

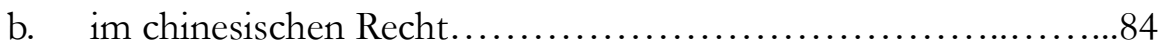

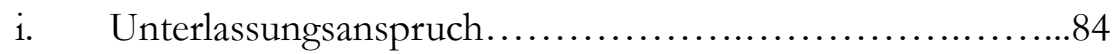

ii. Schadensersatzanspruch................................. 84

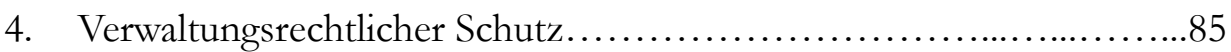

5. Strafrechtlicher Schutz............................................ 85

a. im deutschen Recht....................................... 85

b. im chinesischen Recht...................................... 86

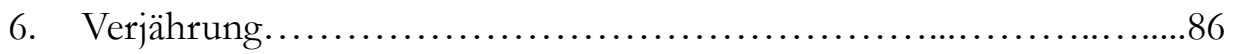

a. im deutschen Recht....................................... 86

b. im chinesischen Recht......................................86

III. Schutz des Urheberrechts............................................... 87

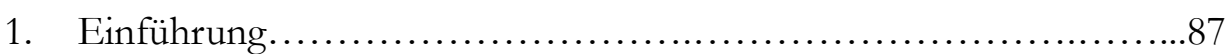

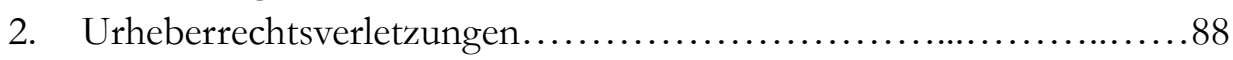

a. im deutschen Recht...................................... 88

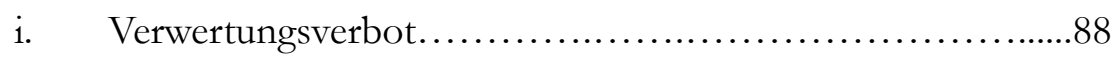

ii. Schutzgegenstand ....................................... 88

iii. Aktivlegitimation........................................ 88 


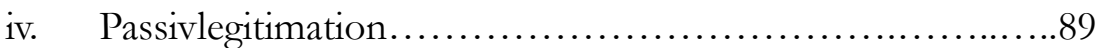

v. Widerrechtlichkeit der Verletzung.........................89

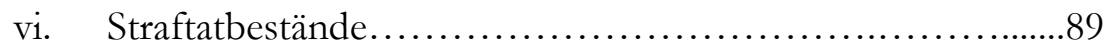

b. im chinesischen Recht.........................................89

3. Zivilrechtlicher Schutz.........................................90

a. im deutschen Recht.................................... 90

i. Beseitigungsanspruch...................................90

ii. Unterlassungsanspruch...............................90

iii. Schadensersatzanspruch................................90

iv. Herausgabe des Gewinns und Auskunfts- und Rechnungslegungsanspruch................................90

v. Vernichtung- und Überlassungsanspruch.....................91

b. im chinesischen Recht.......................................91

i. Anspruchsgrundlage nach Art. 46 ff. chin. UrG................91

ii. Anspruchsgrundlage nach $\iint \quad 23,24$ chin. Computersoftware-VO.................................91

4. Verwaltungsrechtlicher Schutz...................................92

5. Strafrechtlicher Schutz............................................92

a. im deutschen Recht........................................92

b. im chinesischen Recht.....................................92

6. Verjährung................................................ 93

a. im deutschen Recht.....................................93

b. im chinesischen Recht.....................................93

C. Der Schutz des Know-how.........................................93

I. im deutschen Recht............................................93

1. Der zivilrechtliche Schutz.........................................93

a. Geheimnisverrat..........................................93

b. Vorlagenfreibeuterei nach $₫ 18$ UWG...........................94

2. Der strafrechtliche Schutz..........................................94

II. im chinesischen Recht.................................................94

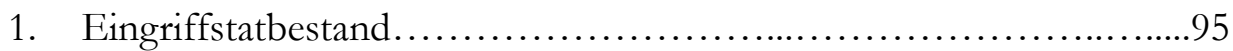

2. Rechtsfolge............................................. 95

\section{Rechtsdurchsetzung zum Schutz des geistigen Eigentums...................................................95}

I. Vorläufige Schutzmaßnahmen.................................... 95

1. im deutschen Recht............................................95

2. im chinesischen Recht.......................................96

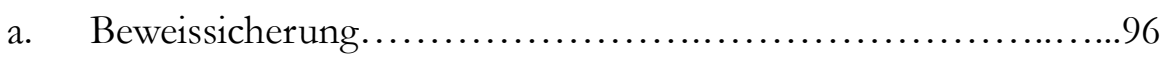

c. Vorwegvollstreckung.........................................96

II. Grenzbeschlagnahme durch den Zoll................................97 
1. im deutschen Recht...........................................97

a. Beim Patentrecht und Gebrauchsmusterrecht....................97

b. Beim Markenrecht........................................97

c. Beim Urheberrecht.........................................97

2. im chinesischen Recht........................................97

a. Einführung........................................... 97

b. Eintragung der Schutzrechte..............................98

c. Beschlagnahme.......................................98

d. Rechtsfolge............................................98

E. Zusammenfassung............................................... 98

Vierter Teil: Vertragrechtliche und kartellrechtliche Grundlagen zum Technologietransfer........................................99

A. Einführung in die Problematik......................................99

I. Der Verhaltenskodex von UNCTAD ..................................100

1. Grundzüge...................................................

2. Die Wettbewerbsregelungen des Verhaltenskodex....................100

II. Das internationale Kartellrechtsabkommen..............................101

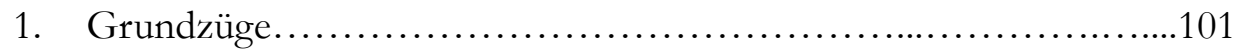

2. Grundprinzipien des IAC.................................. 101

3. Mindestanforderungen des IAC .............................102

B. Die Rechtsbeziehungen im Vertrag über den Technologietransfer.......102

I. Vertrag zur Übertragung von Patentrechten und Rechten zur Patentanmeldung..............................................102

1. Rechte und Pflichten des Veräußerers von Patent und Patentanmeldungsrecht...................................102

a. Übertragung des Vertragsgegenstandes auf den Erwerber........102

b. Haftung............................................ 103

i. Rechtsmängelhaftung.................................103

ii. Sachmängelhaftung...................................103

c. Wettbewerbsverbot........................................104

d. Nichtangriffsabrede........................................104

2. Rechte und Pflichten des Erwerbers von Patent und Patentanmeldungsrecht.........................................104

a. Pflicht zur Zahlung des Kaufpreises.........................104

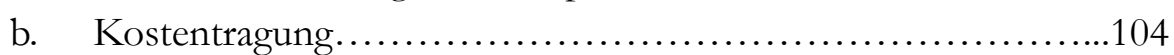

II. Patentlizenzvertrag.............................................104

1. Rechte und Pflichten des Lizenzgebers...........................104

a. Nutzungseinräumung..................................105 
b. Aufrechterhaltung und Verteidigung des Schutzrechts.... 105

i. Anmeldungs- und Aufrechterhaltungspflicht sowie Kostentragung.......................................105

ii. Unterlassungspflicht des Verzichts auf das Schutzrecht.........................................105

iii. Verteidigungspflicht des lizenzierten Schutzrechts........................................106

c. Gewährleistungspflichten.............................106

i. Gewährleistung für industrielle Verwertbarkeit und wirtschaftliche Eigenschaften...........................107

ii. Gewährleistung für Sachmängel (Tauglichkeitsmängel)......107

iii. Gewährleistung für Rechtsmängel......................108

2. Rechte und Pflichten des Lizenznehmers..........................109

a. Pflicht zur Zahlung der Lizenzgebühr........................109

b. Pflicht zur Rechnungslegung..............................109

c. Ausübungspflicht (Best-Efforts-Klausel).....................110

d. Bezugspflichten.....................................112

e. Übermittelung von Verbesserungserfindungen...................112

f. Nichtangriffsabrede....................................112

g. Nachvertragliche Pflichten.................................113

III. Know-how-Vertrag..............................................114

1. Rechte und Pflichten des Know-how-Gebers........................114

a. Die Verpflichtung zur Mitteilung oder Überlassung des Know-how............................................114

b. Haftung.............................................. 114

c. Unterlassung der eigenen Benutzung und Weitergabe des Know-how..........................................115

d. Mitteilungs- und Verbesserungspflichten...........................115

2. Rechte und Pflichten des Know-how-Nehmers......................115

a. Vergütungspflicht des Know-how-Nehmers..................115

b. Geheimhaltungspflicht des Know-how-Nehmers.................116

c. Ausübungspflicht...........................................116

d. Verpflichtungen des Know-how-Nehmers nach Beendigung des Vertrages..............................................116

C. Das neue chinesische Kartellgesetz im Vergleich zum EU-Kartellrecht........................................................117

I. Zweck...............................................................

II. Anwendungsbereich...........................................117

III. Verbot wettbewerbsbeschränkender Vereinbarungen......................118

1. Horizontalvereinbarungen....................................118

2. Vertikalvereinbarungen......................................119

3. Freistellung.................................................119 
IV. Verbot des Missbrauchs einer marktbeherrschenden Stellung.............120

1. Missbrauchstatbestände....................................120

2. Marktbeherrschende Stellung................................121

a. Begriff............................................121

b. Kriterien..........................................121

c. Vermutungstatbestände.....................................121

D. Gruppenfreistellungsverordnung für Technologietransfer -Vereinbarungen....................................................

I. Einleitung.................................................... 122

II. Anwendungsbereich.........................................122

III. Freistellung...............................................122

IV. Marktanteilsschwellen.............................................123

V. Kernbeschränkungen.........................................123

1. Preisbindungsverbot........................................ 123

2. Beschränkung des Outputs....................................123

3. Aufteilung von Kunden oder Märkten..........................124

VI. Nicht freigestellte Beschränkungen.................................124

1. Rücklizenzierungsverpflichtung...............................124

2. Nichtangriffsverpflichtung..................................125

3. Wettbewerbsverbote nicht konkurrierender Unternehmen...........125

VII. Entzug des Rechtsvorteils......................................125

VIII. Nichtanwendbarkeit der GVO-TT..............................125

IX. Freistellung im Einzelfall........................................125

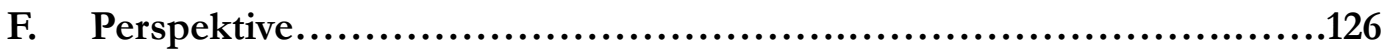

Zusammenfassung.......................................................127

Antimonopolgesetz der VR China........................................131

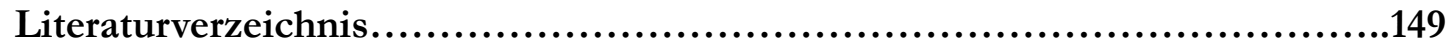




\section{Abkürzungsverzeichnis}

\begin{tabular}{|c|c|}
\hline AGZR & Allgemeine Grundsätze des Zivilrechts \\
\hline AHG & Außenhandelsgesetz \\
\hline Art. & Artikel \\
\hline BGB & Bürgerliches Gesetzbuch \\
\hline BGBl & Bundesgesetzblatt \\
\hline BGH & Bundesgerichtshof \\
\hline BGHZ & Entscheidung des Bundesgerichtshofs in Zivilsachen \\
\hline $\mathrm{BRu}$ & Beijing Rundschau (Zeitschrift, Beijing) \\
\hline BVerfG & Bundesverfassungsgericht \\
\hline bzw. & beziehungsweise \\
\hline ca. & circa \\
\hline chin. & Chinesisch \\
\hline CLP & China Law and Practice (Zeitschrift, Hong Kong) \\
\hline $\mathrm{CPA}$ & $\begin{array}{l}\text { Chinesisches Patentamt (jetzt Chinesisches Amt für geistiges } \\
\text { Eigentum) }\end{array}$ \\
\hline ders. & derselbe \\
\hline d. h. & das heißt \\
\hline EG & Europäische Gemeinschaft \\
\hline EGBGB & Einführungsgesetz zum Bürgerlichen Gesetzbuch \\
\hline EGV & Vertrag zur Gründung der Europäischen Gemeinschaft \\
\hline etc. & et cetera \\
\hline EuGH & Gerichtshof der Europäischen Gemeinschaft \\
\hline f. ff. & folgende, fortfolgende \\
\hline FIE & $\begin{array}{l}\text { Foreign Invested Enterprise, Unternehmen mit ausländischen } \\
\text { Investitionsanteilen }\end{array}$ \\
\hline Fußn. & Fußnote \\
\hline GATS & $\begin{array}{l}\text { Allgemeines Übereinkommen über den Handel mit } \\
\text { Dienstleistungen (General Agreement on Trade in Services) }\end{array}$ \\
\hline GATT 1947 & $\begin{array}{l}\text { Allgemeines Zoll- und Handelsabkommen } 1947 \text { (General } \\
\text { Agreement on Tarifs and Trade 1947) }\end{array}$ \\
\hline GATT 1994 & $\begin{array}{l}\text { Allgemeines Zoll- und Handelsabkommen } 1994 \text { (General } \\
\text { Agreement on Tarifs and Trade 1994) }\end{array}$ \\
\hline GebrMG & Gebrauchsmustergesetz \\
\hline GeschmMG & Geschmacksmustergesetz \\
\hline ggf. & gegebenenfalls \\
\hline GPÜ & Gemeinschaftspatentübereinkommen \\
\hline GRUR & Gewerblicher Rechtsschutz und Urheberrecht \\
\hline GRUR Int. & Gewerblicher Rechtsschutz und Urheberrecht, Internationaler \\
\hline
\end{tabular}


GUG

GVO-TT

GWB

GWY GB

HGB

h. M.

HMA

Hrsg.

IAC

IHK, ICC

IHVB

i. S. d.

i. V. $\mathrm{m}$.

KUG

MMA

MOC

MOFTEC

Nr.

NVK

OECD

PatG

PCT

PVÜ

RBÜ

RIW

Rn.

RMRB

S.

SDRC

SETC

sog.

StGB

TIEVB
Teil

Gesetz der VR China über Gemeinschaftsunternehmen mit chinesisch- ausländischer Kapitalbeteiligung

Gruppenfreistellungsverordnung TechnologietransferVerordnungen

Gesetz gegen Wettbewerbsbeschränkungen

Zhonghua Renmin Gongheguo Guowuyuan Gongbao (Amtsblatt des Staatsrates von China)

Handelsgesetzbuch

herrschende Meinung

Haager Musterabkommen

Herausgeber

International Antitrust Code

Internationale Handelskammer, International Chamber of Commerce

Industrie- und Handelsverwaltungsbehörde von China

im Sinne des

in Verbindung mit

Gesetz der VR China über chinesisch-ausländische Kooperationsunternehmen

Madrider Abkommen über die internationale Registrierung von Marken vom 14. 4. 1891

Handelsministerium (Ministry of Commerce)

Ministerium für Außenhandel und Wirtschaftliche Zusammenarbeit (Ministry of Foreign Trade and Economic Cooperation )

Nummer

Nationaler Volkskongress der VR China

Organization for Economic Cooperation and Development

Patentgesetz

Patentzusammenarbeitsvertrag (Patent Cooperation Treaty)

Pariser Verbandsübereinkunft

Berner Übereinkunft

Recht der internationalen Wirtschaft

Randnummer

Renmin Ribao (Volkszeitung, Beijing)

Seite

Staatliche Kommission für Entwicklung und Reform (State Development and Reform Commission)

Staatliche Kommission für Wirtschaft und Handel (State Economic and Trade Commission)

sogenannte, sogenannter

Strafgesetzbuch

Verwaltungsbestimmungen für den Technologieimport und 
-Export

TIVB

TOT-Code

TRIMs

TRIPs

u. a.

UNO

UNCITAL

UNCTAD

UrhG

u. U.

UWG

VG

vgl.

VO

Vol.

VR

WFOE

WIPO

WIPR

WTO

z. B.

Ziff.

ZK

ZPG

ZPO
Bestimmungen über Verwaltung von Technikimportverträgen Verhaltenskodex für den Technologietransfer, Draft International Code of Conduct on the Transfer of Technology

Übereinkommen über handelsbezogene

Investitionsmaßnahmen (Agreement on Trade-Related Investment Measures)

Übereinkommen über handelsbezogene Aspekte der Rechte des geistigen Eigentums (Agreement on Trade Related Aspects of Intellectual Property Rights)

unter anderem

United Nations

United Nations Commission on International Trade Law

United Nations Conference on Trade and Development

Urhebergesetz

unter Umständen

Gesetz gegen unlauteren Wettbewerb

Vertragsgesetz der VR China

vergleiche

Verordnung

Volume

Volksrepublik

Wholly Foreign Owned Enterprise

World Intellectual Property Organization

World Intellectual Property Report

Welthandelsorganisation

zum Beispiel

Ziffer

Zentralkomitee

Zivilprozessgesetz

Zivilprozessordnung 


\section{Einführung}

\section{Hintergrund der Untersuchung}

Wirtschaftliches Wachstum, ein allgemeiner Wohlstand der Bevölkerung und die Bewältigung der drängenden Probleme in China können nur durch eine das ganze Land erfassende Industrialisierung und Modernisierung erreicht werden. ${ }^{1}$ Dies setzt voraus, dass Wissenschaft und Technologie ein relativ hohes Niveau erreichen können, damit ein nachhaltiges wirtschaftliches Wachstum unterstützt und der Lebensstandard der Bevölkerung ständig verbessert werden kann. Der kürzeste Weg, die Wirtschaft unseres Landes sowie Wissenschaft und Technik zu entwickeln, liegt im Import fortschrittlicher Wissenschaften und Technologien, die den Verhältnissen unseres Landes entsprechen. ${ }^{2}$

Die Entwicklung der Regelungen zum Technologietransfer hat der Wandel der wirtschaftspolitischen Hintergründe Chinas zur Folge. Unter dem Einfluss von diesem Wandel von der zentralen Planwirtschaft zur durch Einführung der Reform- und Öffnungspolitik entstehenden sozialistischen Marktwirtschaft haben sich die Regelungen zum Technologietransfer komplett geändert. Deswegen ist es notwendig, die wirtschaftspolitischen Hintergründe ${ }^{3}$ in Betracht zu ziehen.

\section{Die bisherigen Regelungen zum Technologietransfer}

\section{In Zeiten der zentralen Planwirtschaft}

Nach Art. 5, 15 der ersten Verfassung der VR China am 20. September 19544, Art. 5, 10 der Verfassung von $1974^{5}$ und Art.5, 11 der Verfassung von $1978^{6}$ sollte die Wirtschaftsordnung der VR China auf dem sozialistischen Gemeineigentum an Produktionsmitteln gründen, bestehend aus Volkseigentum und

\footnotetext{
1 Huber, S. V.

${ }^{2}$ Wu, GRUR Int. 1983, S. 624.

${ }^{3}$ Siehe dazu zur Hintergründe der Neuregelungen zum Technologietransfer, Duscha, S. 10 ff.; Glatter, S. 3 ff.; Steinmann, S. 5 ff.; Bohnet, S. 11 ff.;

${ }^{4}$ Diese Verfassung wurde vom 1. Nationalen Volkskongress der VR China (im folgenden: NVK) auf seiner ersten Sitzung verabschiedet, Zhongguo Falü Fagui Xinxi Xitong im Internet unter http://202.99.23.199/home/begin1.cbs; bisher hat China insgesamt 4 Verfassungen erlassen, nämlich die Verfassung von 1954, die Verfassung von 1975, die Verfassung von 1978 und die Verfassung von 1982.

${ }^{5}$ Verabschiedet am 17. Januar 1975 vom 4. NVK auf seiner ersten Sitzung, Zhongguo Falü Fagui Xinxi Xitong im Internet unter http://202.99.23.199/home/begin1.cbs.

${ }^{6}$ Verabschiedet am 5. März 1978 vom 5. NVK auf seiner ersten Sitzung, Zhongguo Falü Fagui Xinxi Xitong im Internet unter http://202.99.23.199/home/begin1.cbs
} 
Kollektiveigentum. ${ }^{7}$ Zusammenfassend ist zu sagen, dass seit Gründung der VR China am 01. Oktober 1949 bis zu der sog. Reform- und Öffnungspolitik von 1978 eine nach dem Vorbild der damaligen UdSSR errichtete starre zentrale Planwirtschaft vorherrschte, die auf der Vorstellung gründete, dass Waren, Güter oder Arbeitsprodukte ganz nach dem Staatsplan verteilt werden sollten. ${ }^{8}$

\section{Nach der Einführung der Reform- und Öffnungspolitik von 1978}

Ende der 70er Jahre beendete China die Kulturrevolution und seine Autarkiepolitik. Am 4. Dezember 1982 verabschiedete der 5. Nationale Volkskongress der VR China auf seiner fünften Sitzung die neue Verfassung ${ }^{9}$, um die Reform- und Öffnungspolitik umzusetzen. Damals schrieb die Verfassung zwar noch vor, dass der Staat auf der Grundlage des Gemeineigentums eine Planwirtschaft aufzubauen und durch eine umfassende Ausbalancierung der Wirtschaftspläne und ergänzend dazu mittels der Marktregulierung eine ausgeglichene und koordinierte Entwicklung der Volkswirtschaft zu sichern hatte, 10 aber wegen der kontinuierlichen Vertiefung der Reform- und Öffnungspolitik wurde diese neue Verfassung durch die zweite Verfassungsänderung am 29. März 1993 vom 8. NVK auf seiner ersten Sitzung revidiert. ${ }^{11}$ Nach Art. 15 Abs. 1 der revidierten Verfassung ist ein sozialistisches Marktwirtschaftssystem in China errichtet worden.

\section{Der heutige Entwicklungsstand Chinas nach dem Beitritt zur Welthandelsorganisation 2001}

Im Zuge der Anpassung an das Regelwerk der WTO mussten in einem noch nie da gewesenen Umfang Gesetze und Verordnungen abgeschafft, neu erschaffen oder geändert werden. ${ }^{12}$ Entsprechend dem Inländerbehandlungsprinzip und Transparenzprinzip hat die VR China die Gesetze und Bestimmungen zum Schutz des geistigen Eigentums überarbeitet und reformiert. Auch die Regelungen zum internationalen Technologietransfer wurden vollständig neu gefasst. Deren Umsetzung wird nach dem Transparenzprinzip liberaler und transparenter.

\section{Gegenstand und Ziel der Untersuchung}

Der Gegenstand dieser Untersuchung ist - wie im Thema dargestellt - die Steuerung des Technologietransfers in der VR China. Dabei ist zu beachten, dass der Schwerpunkt dieser Untersuchung aus wirtschaftsverwaltungsrechtlicher

\footnotetext{
${ }^{7}$ Shan, Xiaoguang, Patentrecte und Know-how im Rechtsverkehr in der Volksrepublik China, S.7.

8 Shan, Xiaoguang, Patentrecte und Know-how im Rechtsverkehr in der Volksrepublik China, S.8; näheres bei Ma, Hong (Hrsg.), S. 39 ff.(41).

${ }^{9}$ GWY GB 1982 Nr. 20 S. 851 ff.; Zhongguo Falü Fagui Xinxi Xitong im Internet unter http://202.99.23.199/home/begin1.cbs; viermal geändert vom NVK am 12. April 1988, 29. März 1993, 15. März 1999, 14. März 2004.

10 Art. 15 Abs. 1 der Verfassung von 1982, GWY GB 1982 Nr. 20 S. 851 f.; Shan, Xiaoguang, Patentrecte und

Know-how im Rechtsverkehr in der Volksrepublik China, S. 11.

${ }^{11}$ Quelle: Zhongguo Falü Fagui Xinxi Xitong im Internet unter http://law.npc.gov.cn:87/home/begin1.cbs

12 Lauffs, S. 13.
} 
Perspektive darauf gelegt wird, wie die chinesische Regierung den Technologietransfer steuert.

Als Gegenstand der Steuerung wird zuerst der Technologietransfer im juristischen Sinne definiert. Es ist herauszustellen, dass der Technologietransfer alle möglichen Typen vom geistigen Eigentum zum Gegenstand hat. Außerdem bestehen die Formen des Technologietransfers aus einer großen Vielfältigkeit. Aber im wesentlichen Teilen geschieht Technologietransfer mit Hilfe von Lizenzverträgen. ${ }^{13}$ Deshalb wird in dieser Untersuchung viel Wert auf den Patentund Know-how-Lizenzvertrag gelegt, dem große Bedeutung in der Rechtspraxis zukommt.

Bei der Untersuchung wird der Technologietransfer aus fünf Perspektiven betrachtet, nämlich, der Interessenlage beim Technologietransfer, der Gesetzgebung zum Technologietransfer, dem Schutz des geistigen Eigentums und den Rechtsbeziehungen der Vertragsparteien sowie der Wettbewerbspolitik.

Zuerst wird die Interessenlage Chinas als Entwicklungsland beim Technologietransfer besprochen. Zu erklären ist Chinas Standpunkt bei der Gesetzgebung zum Technologietransfer und der Rechtspolitik zum Schutz des geistigen Eigentums sowie Bearbeitung der Wettbewerbspolitik (Zweiter Teil, A).

Es fragt sich dann, unter welchen Rechtsrahmen der Technologietransfer durchgeführt werden soll. Die Rechtsvorschriften zum Technologietransfer werden in zwei Bereichen aufgeteilt, nämlich, den zivilrechtlichen Bereich und den wirtschaftsverwaltungsrechtlichen Bereich (Zweiter Teil, B).

Beim Technologietransfer spielen die Schutzrechte des geistigen Eigentums auch eine sehr wichtige Rolle. In diesem Kapitel wird über die Grundzüge des Rechtsschutzes des geistigen Eigentums gesprochen, insbesondere über den Schutz des Patentrechts sowie die Rechtsbeziehung zwischen Rechtsschutz des geistigen Eigentums und Kartellrecht (Dritter Teil).

Folgend sind Pflichten des Schutzrechtsveräußerers und Schutzrechtserwerbers bei den Schutzrechtsveräußerungsverträgen, Pflichten des Lizenzgebers und Lizenznehmers bei den Lizenzverträgen sowie Pflichten des Know-how-Gebers und Know-how-Nehmers bei den Know-how-Verträgen zu erläutern (Vierter Teil, B).

Zum Schluss der Untersuchung werden kartellrechtliche Rechtsbestimmungen beim Technologietransfer behandelt. Darin wird das neue chinesische Kartellgesetz im Rechtsvergleich mit dem EU-Kartellrecht behandelt (Vierter Teil, C, D).

Das Ziel dieser Untersuchung ist, durch die Untersuchung der Rechtslage beim Technologietransfer in China heutzutage und den Rechtsvergleich mit dem Rechtssystem in Deutschland und der Europäischen Union in diesem Bereich, neue Perspektive für die zukünftige Entwicklung des Rechts des Technologietransfers in China zu schaffen.

\footnotetext{
${ }^{13}$ Vgl. Pfaff/Osterrieth, S. 2.
} 


\section{Erster Teil: Technologietransfer}

\section{A. Begriffserklärung}

Da China als Entwicklungsland Technologie aus den westlichen entwickelten Ländern für seine wirtschaftliche und gesellschaftliche Entwicklung benötigt, wird der Technologietransfer ${ }^{14}$ oft in China als Technologieeinfuhr betrachtet. In $\int 2$ der Verwaltungsbestimmungen für den Technologieimport und -Export wird der Begriff „Technologietransfer“ so definiert: Ein- und Ausfuhr von Technologie im Sinne dieser Bestimmungen ist der Transfer von Technologie von einem Gebiet außerhalb der VR China in das Gebiet der VR China hinein oder aus dem Gebiet der VR China heraus, sei es durch Handel, Investition oder technische Zusammenarbeit. Umfasst werden von dem Begriff des Technologietransfers z.B. die Übertragung von Patentrechten, die Abtretung des Rechts zur Patentanmeldung, zur Patentdurchführung, den Transfer des technischen Wissens sowie den Transfer von Technologie durch technische Dienstleistungen in sonstiger Weise. ${ }^{15}$ Die Übertragung von Technologie als Sacheinlage in die Unternehmen mit ausländischen Investitionsanteilen wird auch eingeschlossen. Sogar die Übertragung von Markenrechten im Zusammenhang mit anderen technischen Schutzrechten und vor allem Software sowie das Franchising können einen solchen Transfer darstellen. ${ }^{16}$

Allerdings ist hier zu bemerken, dass in der Praxis die Übertragung des technischen Wissens durch die freie Verfügbarkeit der Informationen zum Wohle der gesamten Gesellschaft gelingt. Ein solcher Transfer der wissenschaftlichen Erkenntnisse wird von der Untersuchung nicht behandelt.

Die OECD, deren Mitglied viele entwickelte Staaten sind, hat Vorschlag ${ }^{17}$ während der Verhandlung für einen internationalen Verhaltenskodex für den Technologietransfer 18 gemacht. Letztlich im Verhaltenskodex wird

\footnotetext{
${ }^{14}$ Siehe dazu zur Volkswirtschaftlichen Funktion des Technologietransfers, Täger, Der Technologietransfer in der BRD, S. 33 ff.

${ }^{15}$ Gabriele Jacob, Das chinesische Außenhandelsrecht für den Güterhandel im Lichte der WTO, Anhang: Bestimmungen der Volksrepublik China über die Verwaltung der Ein- und Ausfuhr von Technik in: Robert Heuser/Roland Klein Die WTO und das neue Ausländerinvestitions- und Außenhandelsrecht der VR China; Siehe dazu, Ganea/Pattloch, S. 274.

${ }^{16}$ Chiang Lingli, Patent World February 2002, 25; vgl. § 6 Nr. 5 der am 20.2. 2002 von MOFTEC und der State Administration of Foreign Exchange verkündeten Mitteilung zur Stärkung der Verwaltung der Devisenzahlung bei Technologieimportverträgen.

${ }^{17}$ Proposal made during the negotiations for an International Code of Conduct on the Transfer of Technology, U.N. Doc. TD/CODE TOT/C.1 WGI/CRP.3 (1979).

${ }^{18}$ UNCTAD (1985). „Draft International Code of Conduct on the transfer of technology, as at the close of sixth
} 
Technologietransfer als die Übertragung der für die Herstellung eines Produkts, die Verwendung eines Verfahrens oder die für die Erbringung und Ausführung einer Dienstleistung notwendigen Kenntnisse definiert. ${ }^{19}$

Aus europäischer Sicht wird der Technologietransfer durch einen Beschluss einer Unternehmensvereinbarung oder eine aufeinander abgestimmte Verhaltensweise vereinbart. Nach Gruppenfreistellungs- Verordnung für Technologietransfer-Vereinbarungen ${ }^{20}$ gehören dazu Patentlizenzvereinbarung, Know-how-Vereinbarung, Softwarelizenz- Vereinbarung oder gemischte Patentlizenz-, Know-how- oder Softwarelizenz-Vereinbarungen einschließlich Vereinbarungen mit Bestimmungen, die sich auf den Erwerb oder Verkauf von Produkten beziehen oder die sich auf die Lizenzierung oder die Übertragung von Rechten an geistigem Eigentum beziehen, sofern diese Bestimmungen nicht den eigentlichen Gegenstand der Vereinbarung bilden und unmittelbar mit der Produktion der Vertragsprodukte verbunden sind. Als Technologietransfer-Vereinbarung gilt auch die Übertragung von Patent-, Know-how- oder Software-Rechten sowie einer Kombination dieser Rechte, wenn das mit der Verwertung der Technologie verbundene Risiko zum Teil beim Veräußerer verbleibt.

\section{B. Gegenstand des Technologietransfers}

Da Technologie ${ }^{21}$ kein juristischer Begriff ist, bedarf sie einer klaren Erklärung, was man unter Technologie verstehen soll. Um Staaten bei ihrer technischen Entwicklung zu helfen, haben die Vereinten Nationen eine sehr weite Auffassung von Technologie akzeptiert, nämlich, „eine Kombination von Einrichtung und Erkenntnis“. ${ }^{22}$ Der Gegenstand des Technologietransfers wird als geistiges Eigentum ${ }^{23}$ bezeichnet. Einen praktisch brauchbaren Ausgangspunkt für die Definition des „geistigen Eigentums“ bietet Art. 2 viii des Übereinkommens zur Errichtung der Weltorganisation für geistiges Eigentum. ${ }^{24}$ Dazu gehören:

session of Conference on June 1998” (Geneva: United Nations), United Nations publication, No. TD/Code TOT/47, 20. June.

${ }_{19}$ Osterrieth, Christian, Die Neuordnung des Rechts des internationalen Technologietransfers, S. 44.; UNCTAD, Draft International Code of Conduct, Chapter 1. para. 1.2.

${ }^{20}$ Verordung (EG) Nr. 772/2004 der Kommission vom 27. 4. 2004 über die Anwendung von Art. 81 Abs. 3 EG-Vertrag auf Gruppen von Technologietransfer-Vereinbarungen, ABl. EG 2004 Nr. L 123/11. Die GVO-TT ist am 1. Mai 2004 in Kraft getreten. Ab dem 31. März 2006 wird die GVO-TT auch auf Vereinbarungen anwendbar sein, die am 30. April 2004 bereits in Kraft waren und die Voraussetzungen der Freistellung nach der GVO-TT (alt) erfüllten Altverträge müssen bis zu diesem Stichtag daher auf ihre Übereinstimmung mit den neuen Regelungen überprüft und gegebenenfalls angepasst werden. Die GVO-TT gilt bis zum 30. April 2014. Siehe auch die gleichzeitig erlassenen Leitlinien zur Anwendung von Art. 81 EG-Vertrag auf Technologietransfer-Vereinbarungen, Bekanntmachung der Kommission, ABl. EG 2004 Nr. C 101/2.

${ }^{21}$ Funke, S. 6 ff.

22 „Equipment comprises all kinds of tools, vehicles, machinery, buildings and what is known as process technology. Technological knowledge covers all kinds of skills... process and product Know-how, institutional and organizational Know-how, and information about equipment and knowledge...” Planning the Technological Transformation of Developing Countries. U.N.Doc. TD/B/C.6/50 (1981).

${ }^{23}$ Vgl. zur Lehre vom geistigen Eigentum, Troller, Immaterialgüterrecht I, S. 20 ff.; Götting, Horst-Peter. Der Begriff des Geistigen Eigentums, GRUR 2006 Heft 5. S. 353 ff. „Das Recht des Geistigen Eigentums schützt verselbstständigte geistige Güter, die dem Rechtsinhaber dem Sacheigentum vergleichbare

Ausschließlichkeitsrechte gewähren.“; Wadle, Geistiges Eigentum I, S. 19 ff.; Rehbinder, Rn. 21 ff. S. 9 ff.

${ }^{24}$ Unterzeichnet am 14. Juli 1967 in Stockholm und geändert am 28. September 1979 
a. Literatur, künstlerische und wissenschaftliche Werke;

b. Leistungen der leistenden Künstler, Telefontelegramm und Rundfunk;

c. Erfindungen auf allen Feldern der menschlichen Bemühungen;

d. Wissenschaftliche Entdeckungen;

e. Industriedesigns

f. Marken, Dienstleistungsmarken sowie gewerbliche Namen und Bezeichnungen;

g. Schutz gegen unlauteren Wettbewerb;

Und alle anderen Rechte aus geistiger Tätigkeit auf den industriellen, wissenschaftlichen oder künstlerischen Feldern. Wenn man sich mit dem Transfer von geistigem Eigentum befassen will, das der Dispositionsbefugnis einzelner unterliegt, muss man sich einen Überblick über die Ausgestaltung des geistigen Eigentums verschaffen.

\section{Patent}

Das Patent ist ein subjektives privates Recht, das seine Entstehung dem Erteilungsakt verdankt. ${ }^{25}$ Einhellig wird anerkannt, dass die Patenterteilung dem Patentinhaber nur dann ein Ausschließlichkeitsrecht 26 verschafft, wenn die Erfindung alle vom Gesetz und der Gerichtspraxis geforderten Merkmale aufweist. ${ }^{27}$ Das Patentrecht handelt vom Schutz für Erfindungen ${ }^{28}$, die neu sind, auf erfinderischer Tätigkeit beruhen und gewerblich anwendbar sind. ${ }^{29}$ Der Schutz wird in Form eines staatlichen Ausschließlichkeitsrechts gewährt. ${ }^{30}$

\section{Gegenstand des Patentschutzes}

Der BGH hat im Fall „Rote Taube“ die dem Patentschutz zugängliche Erfindung als eine „Lehre zum planmäßigen Handeln unter Einsatz beherrschbarer Naturkräfte zur Erreichung eines kausal übersehbaren Erfolges “ gekennzeichnet. ${ }^{31}$ Als weiteres Element des Erfindungsbegriffs hat der BGH die Identifizierbarkeit der Erfindung im Sinne einer inhaltlich bestimmten Lehre zum technischen Handeln, die es anderen Sachverständigen ermöglicht, nach ihr zu arbeiten, angesehen. ${ }^{32}$ Da Patente ihrem Inhaber für den Gegenstand der Erfindung ein ausschließliches Recht gewähren und Dritte von der gewerbsmäßigen Benutzung der unter Schutz gestellten Erfindung ausschließen, muss die Erfindung inhaltlich so eindeutig beschrieben sein, dass der Inhaber des Patents und die interessierte Öffentlichkeit erkennen können, welche Erfindung unter Schutz gestellt ist.

http://www.wipo.int/treaties/en/convention/trtdocs_w0029.html\#P50_1504; Schmidt-Diemitz, Rolf, Geistiges Eigentum und entwicklungspolitischer Wissenstransfer, GRURInt 1988 Heft 04. S. 287.

${ }^{25}$ Benkard, § 1 Rn. 2. S. 75.

${ }^{26}$ Siehe dazu zum Ausschließlichkeitsrecht, Troller, Immaterialgüterrecht I, S. 69 ff.; Chrocziel, S. 15 ff.

27 Troller, Immaterialgüterrecht I, S. 453.

${ }^{28}$ Siehe dazu zur Erfindung, Troller, Immaterialgüterrecht I, S. 145 ff.; Tetzner, Leitfaden, S. 13 ff.; Chrocziel, S. 85.;Steinmann, S. 39 ff.; Kohler, S. 83 ff.; Isay, S. 41 ff.

${ }^{29} \S 1$ Deutsches Patentgesetz, Beier Friedrich-Karl, Patent- und Musterrecht, S. 1.

30 Osterrieth, Patentrecht, S. 2.

31 Krasser, S. 119.

32 Busse, Patentgesetz, Rn. 8. S. 42. 
Deshalb rechnet die Frage der eindeutigen Identifizierbarkeit einer Erfindung auch zu den sachlich-rechtlichen Erfordernissen der Patentfähigkeit.

\section{Patentfähigkeit}

Die Patentfähigkeit 33 einer Erfindung erfordert, dass der Erfinder sich eine technische Aufgabe stellt und hierfür eine Lösung gibt. ${ }^{34}$ Die Aufgabe ist das von dem Erfinder erstrebte technische Ziel, um ein wirtschaftliches oder gesellschaftliches Bedürfnis zu befriedigen. ${ }^{35}$ Mit welchen Mitteln der technische Erfolg erzielt werden soll, hat die Lösung der Aufgabe dem Fachmann zu erläutern. ${ }^{36}$ Die technische Aufgabe ist der eine Teil, der durch Hinzutreten des anderen Teils, der Lösung, zur Gesamtheit der Erfindung wird. ${ }^{37}$ Die Patentpraxis hat neben den gesetzlichen Merkmalen der Neuheit und der gewerblichen Verwertbarkeit noch weitere entwickelt, von denen insbesondere zu nennen sind: Technischer Charakter, Offenbarung, Ausführbarkeit, Nützlichkeit, Fortschritt und Erfindungshöhe. ${ }^{38}$

\section{Rolle des Patents beim Technologietransfer}

Das Patent spielt beim Technologietransfer eine sehr wichtige Rolle. Der Wert des Patents für den Technologietransfer liegt indessen weniger in der Bedeutung des Patents als Offenbarung einer technischen Lehre für die Allgemeinheit als vielmehr darin, dass das Patent eine Rechtsposition schafft, die übertragen werden kann, oder an der Nutzungsrechte eingeräumt werden können. ${ }^{39}$ Damit besitzt der Patentinhaber oder der Anmelder ein Verwertungsmonopol. Im Wege der Geltendmachung der Unterlassungsansprüche kann er Dritte von einer Nutzung ausschließen oder aber auch Dritten im Form der Lizenzverträge Verwertungsrechte einräumen. Insofern bietet das Patent die Möglichkeit, modernste Technologie zu einem verkehrsfähigen Gut und damit zu einem Objekt des Rechtsverkehrs zu machen. ${ }^{40}$

\section{Gebrauchsmuster}

Das Gebrauchsmuster stellt ein subjektives Sonderrecht für technische Erfindungen dar und gewährt dessen Inhaber ein befristetes, ausschließliches Benutzungs- und Ausschließungsrecht. ${ }^{41}$ Die Erfindungen müssen neu und gewerblich anwendbar sein sowie auf einem erfinderischen Schritt beruhen, um nach Eintragung - geschützt zu sein. ${ }^{42}$ Die Formulierung „erfinderischer Schritt“ lässt erkennen, dass gegenüber einem Patent ein geringeres Maß an

\footnotetext{
33 Siehe dazu, Ausnahmen von der Patentfähigkeit, Tetzner, Leitfaden, S. 16 ff.; Isay, S. 93 ff.; Voraussetzungen, Chrocziel, S. 86 ff.; Steinmann, S. 69 ff.; Ganea/Pattloch, S. 8 ff.; Holyoak/Torremans, S. 46 ff.; Pedrazzini, Patentrecht, S. 49 ff. 72 ff.; Pedrazzini, S. 14 ff.

${ }^{34}$ Reimer, § 1 Rn. 9. S. 25.

35 Lindenmaier/Weiss, Rn. 20. S. 24.

${ }^{36}$ Lindenmaier/Weiss, Rn. 21. S. 26.

37 Reimer, § 1 Rn. 10. S. 25.

${ }^{38}$ Siehe dazu, Klauer/Möhring, Rn. 19. S. 64.

${ }^{39}$ Osterrieth, Patentrecht, S. 9.

40 Osterrieth, Patentrecht, S. 9.

${ }^{41}$ Loth, Rn. 2. S. 19.; Vgl. Chrocziel, S. 108 ff.

42 Bühring, Rn. 2. S. 6.
} 
Erfindungshöhe ausreicht. ${ }^{43}$ Deswegen spielt das Gebrauchsmuster für den Technologietransfer nur eine untergeordnete Rolle.

\section{Geschmacksmuster}

Als Geschmacksmuster ${ }^{44}$ werden Muster geschützt, die neu sind und Eigenart haben. ${ }^{45}$ Ein Muster ist im Sinne dieses Gesetzes die zweidimensionale oder dreidimensionale Erscheinungsform eines ganzen Erzeugnisses oder eines Teils davon, die sich insbesondere aus den Merkmalen der Linien, Konturen, Farben, der Gestalt, Oberflächenstruktur und der Werkstoffe des Erzeugnisses selbst oder seiner Verzierung ergibt. ${ }^{46}$ Im allgemeinen Sprachgebrauch wird der Begriff „Design“ verwendet, dem dieselbe Bedeutung wie dem in der Rechtssprache verwendete Begriff „Geschmacksmuster“ zukommt. 47 Das Geschmacksmusterrecht gewährt in erster Linie seinem Rechtsinhaber das ausschließliche Recht, das Muster in Verbreitungsabsicht nachzubilden ( $\int S$ 7, 38 Abs. 1 GeschmMG). ${ }^{48}$ Die Nachahmung setzt nicht nur einen objektiven, sondern auch einen subjektiven Tatbestand voraus. Das nachgebildete Muster muss beim informierten Benutzer einerseits denselben Gesamteindruck erwecken wie das Geschmacksmuster (objektive Nachahmung) und der Benutzer muss das Geschmacksmuster gekannt haben (subjektive Nachahmung) (\$ 38 Abs.1, 2 GeschmMG). ${ }^{49}$ Dem Haager Abkommen über die internationale Hinterlegung gewerblicher Muster und Modelle vom 6.November.1925, mit inzwischen einigen Revisionen und Ergänzungen, sind nur wenige Länder beigetreten. ${ }^{50}$ Dem Geschmacksmusterrecht kommt schon aufgrund dieser Situation nur eine eingeschränkte Bedeutung für den Technologietransfer zu.

\section{Markenrechte}

Eine Marke 51 ist ein Zeichen, das im geschäftlichen Verkehr dazu dient, die Waren oder Dienstleistungen eines Unternehmens zu individualisieren ${ }^{52}$ und mit der das Unternehmen deshalb seine Waren und Dienstleistungen kennzeichnet. ${ }^{53}$ In dem Markengesetz $^{54}$ ist unter dem Oberbegriff der „Kennzeichen“ der Schutz der Marken, geschäftlichen Bezeichnungen und geographischen Herkunftsangaben geregelt. ${ }^{55}$ Der Begriff „Marken“ umfasst sowohl angemeldete und eingetragene Marken als auch durch Benutzung erworbene Marken sowie die i. S. v. Art $6^{\text {bis }}$

\footnotetext{
${ }^{43}$ Mes, Rn. 9. S. 722.

44 Vgl. BGH GRUR 1980, 235 - „Play-family“. Die Rechtsprechung definierte das Geschmacksmuster als Gestaltung, die bestimmt und geeignet ist, das geschmackliche Empfinden des Betrachters anzusprechen.

${ }^{45} \S 2$ Abs. 1 GeschmMG, Eichmann, S. 70.; Chrocziel, S. 126 f.

$46 \S 1$ Nr. 1 GeschmMG, Eichmann, S. 55.; Rehmann, Rn. 9. S. 3.

47 Rehmann, S. 3.

48 Vgl. Hubmann/Götting, S. 256.; Rehmann, Anhang I, S. 65.

49 Rehmann, Rn. 151. S. 37, Anhang I, S. 65.; Hubmann/Götting, S. 257.

50 Übersicht über den Stand der internationalen Verträge auf dem Gebiet des gewerblichen Rechtsschutzes am 1. Januar 2004, GRURInt. 2004 Heft 5. S. 398 ff.

${ }^{51}$ Siehe dazu zu den Merkmalen der Marke, Troller, Immaterialgüterrecht I, S. 205 ff.; v. Schultz, § 3 Rn. 1 ff. S.

37 ff.; Ekey/Klippel, S. 84 ff.

52 Fezer, Rn. 30. S. 72.

${ }^{53}$ Nordemann, S. 339.

${ }^{54}$ Siehe dazu Markengesetz, Köhler, S. 79 ff.

55 Berlit, S. 10.
} 
Pariser Verbandsübereinkunft notorisch bekannten Marken. 56 Nach der Rechtssprechung von EuGH und BGH besteht die Hauptfunktion der Marke darin, dem Verbraucher oder Endabnehmer die Ursprungsidentität der gekennzeichneten Ware oder Dienstleistung zu garantieren, in dem sie ihm ermöglicht, diese Ware oder Dienstleistung ohne Verwechslungsgefahr von Waren oder Dienstleistungen anderer Herkunft zu unterscheiden. ${ }^{57}$ Die Vorschrift gewährt dem Inhaber einer Marke ein ausschließliches Recht, der durch die Eintragung eines Zeichens als Marke in das Markenregister, durch die Benutzung eines Zeichens bei Erwerb von Verkehrsgeltung als Marke oder durch die notorische Bekanntheit einer Marke im Sinne von Art. 6bis PVÜ ein Markenrecht erwirbt. ${ }^{58}$

Dem Madrider Abkommen über die internationale Registrierung von Marken vom 14. 4. 189159 mit zahlreichen zwischenzeitlichen Revisionen sind 54 Mitgliedsstaaten beigetreten. ${ }^{60}$ Das MMA ist ein Nebenabkommen der PVÜ; es begründet einen Sonderverband im Rahmen des Pariser Verbandes. ${ }^{61}$ Es erlaubt dem Inhaber einer in einem Vertragsstaat registrierten Marke durch eine einheitliche Registrierung beim internationalen Büro der WIPO in Genf in jedem Vertragsstaat den gleichen Schutz wie durch eine nationale Einzelanmeldung in diesem Vertragsstaat zu erlangen. ${ }^{62}$

Die Verwendung der Marke kann zur Gewährung der Qualität der Waren oder Dienstleistungen und für den Vertrieb eines Produktes von wesentlicher Bedeutung sein. ${ }^{63}$ Beim Technologietransfer hat das Markenrecht allerdings nur eine Nebenrolle gespielt. ${ }^{64}$

\section{Urheberrecht}

Urheberrecht ${ }^{65}$ ist einerseits die Summe aller Rechtsnormen, die den sozialen Tatbestand der Werkherrschaft regeln, andererseits das subjektive Recht des Urhebers an seinem Werk. ${ }^{66}$ Nach $\ 1$ Urhebergesetz sind allgemein Werke der Literatur, Wissenschaft und Kunst urheberrechtlich geschützt. ${ }^{67}$ Der Begriff des Urheberrechts hat zwei Sinne. Im objektiven Sinne regelt das Urheberrecht den Schutz bestimmter kultureller Geistesschöpfungen, die Werke genannt werden. ${ }^{68}$ Im subjektiven Sinne ist Urheberrecht die Berechtigung des Werkschöpfers, des

\footnotetext{
56 Ströbele/Hacker, S. 11.

57 EuGH GRUR 1998, S. 922 f. - Canon; BGH GRUR 1999, S. 731 ff. - Canon II; BGH GRUR 1999, S. 496 f. TIFFANY.

58 Fezer, Rn. 8. S. 631.

59 Siehe dazu zum Schutz von Marken nach MMA und dem Protokol, S. 1588 ff.

${ }^{60}$ Siehe dazu GRURInt. 2004 Heft 5, S. 401 ff.

${ }^{61}$ Busse, Warenzeichengesetz, S. 739.

62 Ingerl/Rohnke, S. 1254.

${ }^{63}$ Vgl. Chrocziel, S. 131 ff. „§ 3 Abs. 1 MarkenG enthält den Grundsatz, dass Marken zur Unterscheidung der eigenen Waren oder Dienstleistungen von den Waren oder Dienstleistungen anderer Unternehmen dienen sollen; es soll eine Unterscheidung nach der betrieblichen Herkunft ermöglicht werden.“

${ }^{64}$ Siehe dazu zur Markenlizenz, v. Schultz, § 30 Rn. 1 ff. S. 490 ff.; Bohnet, S. 179 ff.; Ganea/Pattloch, S. 113 ff.; Stumpf/Groß, S. 285 ff.; Pfaff/Osterrieth, S. 440 ff.

${ }^{65}$ Zur Geschichte des Urheberrechts, Wadle, Geistiges Eigentum Band I, S. 99 ff./Band II, S. 101 ff.; Rehbinder, Rn. 11 ff. S. 6 ff.

${ }^{66}$ Rehbinder, S. 1.

${ }^{67}$ Hillig, S. 3.

${ }^{68}$ Rehbinder, S. 1.
} 
Urhebers, an seinem Geisteswerk. ${ }^{69}$

Nach \11 UrhG schützt das Urheberrecht den Urheber in seinen geistigen und persönlichen Beziehungen zum Werk und in der Nutzung des Werkes. ${ }^{70}$ Das Urheberrecht enthält demnach zwei wesentliche Schutzbereiche, es sichert dem Urheber die Verwertungsrechte an seinem Werk und es schützt den Urheber im Hinblick auf seine ideellen Interessen an seiner Schöpfung. ${ }^{71}$ Sie werden als Urheberpersönlichkeitsrecht ${ }^{72}$ und Verwertungsrecht genannt.

Begriff und Bedeutung des Urheberpersönlichkeitsrechts sind zuerst im französischen Recht erkannt worden (droit moral) und dann auch durch Art. 6 bis $^{\text {is }}$ Revidierte Berner Übereinkunft (seit der Rom-Konferenz 1928) international rechtlich anerkannt worden. ${ }^{73}$ Gegenstand des Urheberpersönlichkeitsrechts ist nicht die Person des Urhebers, die als solche durch das allgemeine Persönlichkeitsrecht geschützt wird, sondern seine Schöpfung, also die Verbindung des Werkes mit seiner schöpferischen Persönlichkeit. ${ }^{74}$ Die Intensität der urheberpersönlichkeitsrechtlichen Interessen bestimmt sich dabei weitgehend nach dem Werkcharakter und der Werkeigenart. 75 Das Urheberpersönlichkeitsrecht ist aus einer Anzahl einzelner urheberpersönlichkeitsrechtlicher Befugnisse gebildet. ${ }^{76}$ Aus dem Urheberrechtpersönlichkeitsrecht fließen insbesondere die Befugnisse des Urhebers, über die Veröffentlichung seines Werkes zu befinden, Anerkennung seiner Urheberschaft $\mathrm{zu}$ verlangen und Entstellungen seines Werkes entgegenzutreten. ${ }^{77}$

Der wichtigste Grundgedanke bei der Ausgestaltung der Verwertungsrechte, der auch für ihre Auslegung maßgebend sein muss, ist der Grundsatz der tunlichst angemessenen Beteiligung des Urhebers an dem wirtschaftlichen Nutzen, der aus seinem Werk gezogen wird. ${ }^{78}$ Nach $\int 15$ UrhG ist dieses den beispielhaft aufgezählten Berechtigungen übergeordnete, vermögensrechtliche Verwertungsrecht das ausschließliche, einheitliche, umfassende Recht zur Verwertung des immateriellen geistigen Werks in körperlicher Form und zu seiner öffentlichen Wiedergabe in unkörperlicher Form. ${ }^{79}$

Völkerrechtliche Regelungen ${ }^{80}$ für das jeweils nationalstaatlich ausgestaltete Urheberrecht enthalten vor allem die Berner Übereinkunft mit derzeit 152

\footnotetext{
${ }^{69}$ Rehbinder, S. 1.

${ }^{70}$ Hillig, S. 5.

${ }^{71}$ Ensthaler, S. 43.; Vgl. Chrocziel, S. $151 \mathrm{ff}$.

72 Siehe dazu zum Urheberpersönlichkeitsrecht, Schricker, S. 256 ff.; Rehbinder, S. 194 ff.; Schack, S. 149 ff.; Loewenheim, S. 191 ff.; Dreyer/Kotthoff/Meckel, S. 174 ff.; Dreier/Schulze, S. 177 ff.; Wandtke/Bullinger, S. 188 ff.; Möhring/Nicolini, S. 250 ff.; Forkel, S. 168 ff.; Fabiani, Mario; Der Schutz der Persönlichkeit im Urheberrecht, Forkel, Hans; Zur Systematischen Erfassung und Abgrenzung des Persönlichkeitsrechts auf Individualität, in: Forkel, Hans/Kraft, Alfons, Beiträge zum Schutz der Persönlichkeit und ihrer schöpferischen Leistungen, S. 87 ff. 93 ff.; Pedrazzini, S. 74.

${ }^{73}$ Fromm/Nordemann, S. 142.

${ }^{74}$ Möhring/Nicolini, S. 245.

75 v. Gamm S. 294.

${ }^{76}$ Wandtke/Bullinger, S. 189.

77 Möhring/Nicolini, S. 245

${ }^{78}$ Vgl. Schricker, S. 343.

${ }^{79}$ v. Gamm S. 325.

${ }^{80}$ Siehe dazu, Internationales Urheberrecht, Delp, S. 344 ff.; Rehbinder, S. 327 ff.
} 
Mitgliedsstaaten und das Welturheberrechtsabkommen, dem 97 Staaten beigetreten sind. 81 Abgesehen von dem Sonderbereich der Computerprogramme ${ }^{82}$ ist das Urheberrecht im Zusammenhang mit dem Technologietransfer nicht von großer Bedeutung. ${ }^{83}$

\section{Know-how}

\section{Begriffserklärung}

Der Begriff Know-how ist im anglo-amerikanischen Rechtskreis entwickelt worden. ${ }^{84}$ Nach der allgemeinen Meinung geht der Inhalt des Begriffs „Know-how“ sehr weit und müsste so eine Definition daher sehr weit gefasst werden. Man kann daher sagen, „technisches Know-how sind alle technischen Kenntnisse und Erfahrungen, die für die industrielle Anwendung nützlich sind und die daher einen wirtschaftlichen Wert darstellen“. ${ }^{85}$ Nach der Definition des BGH ist Know-how eine Tatsache, die nicht offenkundig ist, d. h. nur einem eng umgrenzten Personenkreis mitgeteilt worden ist und die nach dem geäußerten oder erkennbaren Willen des Betriebsinhabers aus berechtigten wirtschaftlichen Interessen geheim gehalten werden soll. ${ }^{86}$ Dabei umfasst der Begriff des technischen Know-how als Oberbegriff das technisch geprägte Betriebsgeheimnis und das nicht geheime Erfahrungswissen. ${ }^{87}$

Die Internationale Handelskammer hat sich um eine Klärung des Begriffs Know-how bemüht und folgende Formulierung im Sitzungsbericht vom 17. und 18. Oktober 1957 entworfen: „Die Gesamtheit der Kenntnisse, des Fachwissens und der Erfahrung bei dem Verfahren und bei der technischen Durchführung der Fabrikation eines Produkts kann daher art de fabrication(=Know-how) genannt werden." 88

Im Sinne des Art. 1 Abs. 1 i) GVO-TT ist Know-how eine Gesamtheit nicht patentierter praktischer Kenntnisse, die durch Erfahrungen und Versuche gewonnen werden und die geheim, wesentlich und identifiziert sind. Es ist heute unbestritten, dass auch Gegenstände, für die kein Schutzrecht erworben werden kann, Dritten durch einen sog. Know-how-Vertrag zur Verwertung überlassen

\footnotetext{
${ }^{81}$ Siehe dazu GRURInt. 2004 Heft 5, S. 407 ff.; Delp, S. 344 ff.

82 Siehe dazu zur Computer-Programme, Delp, S. 332 ff.; Geissler/Pagenberg, S. 629 ff.; Ensthaler, S. 52 ff.; Loewenheim, S. 91 ff.; Rehbinder, S. 95 ff.; Dreyer/Kotthoff/Meckel, S. 855 ff.; Schricker, S. 1327 ff.; Dreier/Schulze, S. 843 ff.; Wandtke/Bullinger, S. 737 ff.; Möhring/Nicolini, S. 675 ff.

83 Allerdings wird das Urheberrecht auch durch Lizenzverträge Dritten eingeräumt, ein urheberrechtlich geschütztes Werk gegen die Vergütungspflicht in der vertraglich bestimmten Weise zu nutzen. Siehe dazu zum Urhebervertragsrecht, Delp, S. 386 ff. „,Das urheberrechtlich geschützte Werk, die durch verwandte Schutzrechte geschützte Leistung soll Dritten vermittelt, vervielfältigt, verbreitet, vorgetragen, ausgestellt, verfilmt, durch Rundfunksendung in Wort und Bild verbreitet oder durch elektronische Medien anderen Menschen zugänglich gemacht werden. Die hierzu benötigten Instrumente liefert das Urhebervertragsrecht.“ S. 392 ff. „Es hat sich im Verlagswesen eingebürgert, als „Lizenzverträge“ die Abmachungen des Originalverlegers mit Dritten über die Nutzung urhebervertragsrechtlicher Nebenrechte zu bezeichnen.“

${ }^{84}$ Siehe dazu zum Know-how im anglo-amerikanischen Rechtskreis, Sehirali, S. 10 ff.; Böhme, S. 12 ff.; Pfister, S.

8 ff.; Stumpf, S. 259 ff.; Dessemontet, S. $11 \mathrm{ff}$.

${ }^{85}$ Fischer, GRURInt. 1970 Heft 07. S. 240

${ }^{86}$ BGH GRUR 1955, 424, 426 - Möbelwachspaste; GRUR 1961, 40, 43 - Wurftaubenpresse.;Vgl. Krasser, GRUR 1970, S. 589.; Krasser, GRUR 1977, S. 178.

${ }^{87}$ Gaul/Bartenbach, S. Q 1.

${ }^{88}$ Stumpf, Der Know-how-Vertrag, S. 21.
} 
werden können. ${ }^{89}$ Im Zusammenhang mit der Übertragung technischen Wissens ist der Begriff des Know-how von zentraler Bedeutung.

\section{Rechtsnatur}

Die Frage, ob an dem Know-how als Gegenstand des Know-how-Vertrages ein absolutes subjektives Recht ${ }^{90}$ besteht, ist umstritten.

Die herrschende Meinung ${ }^{91}$ verneint ein absolutes subjektives Recht, denn das Know-how stellt vielmehr nur einen faktischen Besitz, den tatsächlichen Zugriff auf Kenntnisse, dar. ${ }^{92}$ Es handelt sich bei Übertragungen von geheimem Wissen oder von Know-how sowie bei Lizenzvergabe daran um eine bloß schuldrechtliche Angelegenheit. ${ }^{93}$

Nach Ansicht von Forkel $^{94}$ stellt das Know-how ein absolutes subjektives Recht dar. Forkel meint, dass bei einer denkbaren Übertragung von Kenntnissen nicht einfach das Wissen, das weitergegeben wird, sondern eine Berechtigung das subjektive Recht - an ihm veräußert würde. ${ }^{95}$ Derjenige, an den das Wissen weitergegeben würde, könnte dann sowohl dem bisherigen Berechtigten als auch Dritten gegenüber eine sichere Position erlangen.

Nach Ansicht von Pfister 96 wird Know-how unter den weiten Eigentumsbegriff subsumiert. Das Bundesverfassungsgericht hat in seiner Entscheidung vom 30. 4. 195297 die „konstituierenden Merkmale des Eigentumsbegriffs“ herausgearbeitet: „Es entspricht den heute allgemein herrschenden gesellschaftlichen Auffassungen, das, was der Einzelne sich durch eigene Leistung und eigenen Kapitalaufwand erworben hat, im besonderen Sinne als sein Eigentum anzuerkennen...". 98 In mehreren Entscheidungen hat das BVerfG diese Auffassung ständig bestätigt: Entscheidend ist danach nicht, dass die in Frage stehende Vermögensposition schon anders von der Rechtsordnung als subjektives Recht anerkannt ist, sondern allein, dass sie auf einer eigenen persönlichen oder sachlichen Leistung beruht. ${ }^{99}$

Das Know-how als Gegenstand technischer, betriebswirtschaftlicher und kaufmännischer Kenntnisse und Erfahrungen bildet genauso wie Schöpfungen

\footnotetext{
${ }^{89}$ Stumpf/Groß, Rn. 16. S. 41.; Vgl. dazu Stumpf, Der Know-how-Vertrag, Rn. 1 ff.

90 Siehe dazu zum subjektiven Recht, Riemer, S. 27.; Dessemontet, S. 49 ff.; Meili, S. 19. „Der Begriff „subjektives Recht“ bezeichnet eine dem Einzelnen durch die Rechtsordnung verliehene Rechtsmacht.“

${ }_{91}$ Vgl. Pfaff, BB 1974, S. 567; Stumpf, Der Know-how-Vertrag, Rn. 15. S. 34 ff.; Gitter, S. 445.; Troller, GRURAusl. 1958, S. 385 ff.; Krasser GRUR 1970, S. 587 ff.; Krasser, GRUR 1977, S. 177 ff; Pfister, S. 31 ff.; Hoppe, S. 155 ff.; Benkard, § 15 Rn. 235. S. 615.

92 Anders der BGH im Dücko - Urteil, GRUR 1955, S. 338; er bejaht dort im Ergebnis ein abstraktionsfähiges und übertragbares subjektives Recht an einem Geheimrezept. Er leitete dieses dogmatisch aus dem allgemeinen Persönlichkeitsrecht bzw. dem Recht am eingerichteten und ausgeübten Gewerbebetrieb ab. Das Ergebnis überzeugt jedoch nicht, da es - wie die Urteilsgründe zeigen - vorrangig ergebnisorientiert war. Eine Lücke im Wettbewerbsrecht sollte hier durch die Konstruktion eines subjektiven Rechts geschlossen werden; Pfaff, BB 1974, S. 565; Hoppe, S. 156.

${ }_{93}$ Forkel, FS Schnorr v. Carolsfeld, S. 107.

${ }^{94}$ Forkel, FS Schnorr v. Carolsfeld, S. 121.

95 Vgl. Forkel, FS Schnorr v. Carolsfeld, S. 109, 121.; Hoppe, S. 154.

${ }^{96}$ Pfister, S $42 \mathrm{ff}$.

97 BVerfGE Bd. 1, S. 264.

98 Pfister, S. 43 f.; Vgl. Meili, S. 25.

99 BVerfG v. 29.11.1961 Bd. 13, S. 225, 229. (der Gewerbebetrieb stellt grundsätzlich Eigentum dar), BVerfG v. 11.10.1962 Bd. 14, S. 288 ff., 293 f. und v. 7. 5. 1963 Bd. 16, S. 94. (Versorgungsansprüche ehemaliger Berufssoldaten unterliegen Art. 14 GG, weil sie auf die Leistung des Beamten zurückgehen).
} 
technischer und ästhetischer Natur ein Immaterialgut. ${ }^{100}$ Immaterialgüter sind als Rechtsobjekte nur im Rahmen der gesetzlichen Voraussetzungen und Schranken geschützt (numerus clausus des Gesetzes). 101 Dieser numerus clausus des Gesetzes macht deutlich, dass das Know-how als Immaterialgut nicht von der Rechtsordnung als Rechtsobjekt ausgewählt worden ist. ${ }^{102}$ Deshalb ist der herrschenden Meinung zu folgen, dass kein absolutes subjektives Recht entsteht.

\section{Formen des Technologietransfers}

Der Vielfalt der Erscheinungsformen ${ }^{103}$ entspricht eine fast unbegrenzte Vielfalt der Übertragungsmöglichkeiten. ${ }^{104}$ Es umfasst alle Arten von Übertragungsakten einschließlich Handel, Investitionen oder wirtschaftliche bzw. technische Kooperationen, und meint insbesondere die Übertragung von Patentrechten oder Patentanmelderechten, die Lizenzierung von Patentrechten, die Übertragung geheimen Know-how, technische Dienstleistungen und jeden anderen Transfer von Technologie.

Der Verhaltenskodex hat die Übertragungsformen wie folgt aufgestellt:

a. „die Übertragung, der Verkauf oder die Lizenzierung aller Formen gewerblichen Eigentums außer Handelsmarken, Dienstmarken oder Handelsnamen, wenn sie nicht Teil des Technologietransfervertrages sind;

b. die Übertragung von Know-how, die technische Beratung in Gestalt von Projektstudien, Plänen, Grafiken, Modellen, Hand- und Lehrbüchern, Formularen, die Vermittlung von Design, Ausbildungsplänen, Dienstleistungen einschließlich technischer Anleitung sowie Personalausbildung;

c. die Vermittlung von technischen Wissen, welches für die Errichtung, die Verwendung und die Funktionsfähigkeit von Betriebsanlagen und Errichtungen, sowie für schlüsselfertige Anlagen notwendig ist;

d. die Vermittlung von technischem Wissen, welches für den Erwerb, die Aufstellung und den Gebrauch von Maschinen, Ausrüstungsgegenständen, Zwischenprodukten und/oder Rohmaterialien, erforderlich ist;

e. die Bekanntgabe des Gegenstandes industrieller und technischer Kooperationsverträge. “ 105

Außerdem nennt Stumpf als verschiedene Formen des Technologietransfers:

a. „Übermittlung einschlägiger Literatur;

b. Ausbildung von einheimischen Arbeitskräften;

c. Entscheidung von Technikern, Ingenieuren und Managern;

d. Lieferung von Investitionsgütern und/oder Industrieanlagen (turn-key-contracts; contrat ,produit en main“);

e. Patent-Lizenzverträge;

100 Gitter, S. 445.

101 Vgl. Gitter, S. 445.; Troller, Schutz, S. 217.; Meili, S. 25.

${ }^{102}$ Gitter. S. 445

103 Siehe dazu, Osterrieth, S. 9 ff.; An, S. 138 ff.

104 Osterrieth, S. 9.

${ }^{105}$ UNCTAD, Draft International Code of Conduct, Chapter 1. para. 1.3.; Osterrieth, Christian, Die Neuordnung des Rechts des internationalen Technologietransfers, S. $44 \mathrm{f}$. 
f. Know-how-Verträge;

g. Verträge über technische Hilfe;

h. Direktinvestitionen;

aa. Industrie-Kooperation

bb. Errichtung von Gemeinschaftsunternehmen

cc. Gründung von Tochtergesellschaften. "106

Zum Vertrag über den Technologietransfer nach dem chinesischen Recht gehören Verträge über die Übertragung von Patenten, über die Übertragung des Rechts zur Patentanmeldung, über die Übertragung technischer Geheimnisse (Übertragung des Know-how) und über die Lizenz zur Anwendung von Patenten. ${ }^{107}$ Anschließend werden diese Arten des Vertrags über den Technologietransfer näher untersucht.

\section{Vertrag zur Übertragung von Patentrechten und}

\section{Rechten zur Patentanmeldung}

Nach \15 Abs. 1 Patentgesetz gehen das Recht auf das Patent, der Anspruch auf Erteilung des Patents und das Recht aus dem Patent auf die Erben über und können beschränkt oder unbeschränkt auf andere übertragen werden. ${ }^{108}$ Unter dem Recht auf das Patent ist die Gesamtheit der aus der Erfindung herrührenden Rechte zu verstehen, soweit diese vermögensrechtliche Natur ist. ${ }^{109}$ Der Erfinder kann bereits vor der Einreichung einer Patentanmeldung seine Rechte an der Erfindung übertragen. Die Übertragung setzt jedoch die Fertigstellung der Erfindung voraus. ${ }^{110}$

Grundsätzlich hat der Verkäufer des Patents für den Bestand des verkauften Rechts zur Zeit des Vertragsschlusses einzustehen. Die entgeltliche Übertragung der genannten Rechte ist nach $\int 453$ BGB in der Regel Rechtskauf. ${ }^{111}$ Es macht hinsichtlich des Charakters als Kaufgeschäft keinen Unterschied, ob die Veräußerung eine noch nicht angemeldete Erfindung, den Anspruch auf Erteilung des Patents oder das erteilte Patent selbst betrifft. ${ }^{112}$ In der Regel passiert die Lizenzierung durch den Lizenzvertrag häufig, während der Patentkauf selten ist.

\section{E. Patentlizenzvertrag}

\section{Einführung}

Das Wort „Lizenz“, aus dem Lateinischen „licet“ entsprechend „es ist erlaubt" abgeleitet, hat mit diesem Wortstamm internationale Bedeutung

\footnotetext{
106 Stumpf, GRURInt 1977, S. 441.

107 Vgl. § 342 VG Siehe unten Fn. 31

108 Beier, S. 6.

109 Benkard, § 15 Rn. 2. S. 560.

110 Osterrieth, Patentrecht, Rn. 399. S. 215.

111 Vgl. BGHZ 1983, S. 283, 287 - Hartmetallkopfbohrer; Benkard, § 15 Rn. 24. S. 565.

112 Vgl. RG GRUR 1938, S. 33 f.; Benkard, § 15 Rn. 24. S. 565.
} 
erlangt. ${ }^{113}$ Die Lizenz oder das Lizenzrecht im subjektiven Sinne ist das durch oder gestützt auf einen Vertrag erworbene Recht des Lizenznehmers, während einer bestimmten Dauer in Bezug auf ein absolut geschütztes Immaterialgut Benutzungshandlungen vorzunehmen, die von Gesetzes wegen an sich dem Schutzrechtsinhaber vorbehalten wären. ${ }^{114}$ Lizenzverträge ${ }^{115}$ sind Verträge, durch die der Inhaber eines Schutzrechtes einem anderen die Ausübung an sich dem Berechtigten aufgrund des Schutzrechts vorbehaltener Tätigkeiten gestattet. ${ }^{116}$

\section{Rechtsnatur des Lizenzvertrages ${ }^{117}$}

Der Lizenzvertrag ist grundsätzlich dem allgemeinen Vertragsrecht unterstellt, d. h. er nimmt gegenüber anderen Vertragstypen keine Sonderstellung ein. ${ }^{118}$ Der Lizenzvertrag ist ein Dauerschuldverhältnis, das beiden Vertragspartnern eine gesteigerte gegenseitige Treuepflicht auferlegt. ${ }^{119}$ Maßgebliche gesetzliche Grundlage für den Patentlizenzvertrag ist die Bestimmung des $\ 15$ Abs. 2 PatG, die ausdrückliche Regelung enthält, dass Patentrechte ganz oder teilweise Gegenstand von ausschließlichen oder nichtausschließlichen Lizenzen sein können. ${ }^{120}$ Die Quasi-Übertragung der Patentrechte in Form einer ausschließlichen Lizenz verschafft dem Lizenznehmer die gleiche Rechtsstellung wie dem ursprünglichen Patentinhaber. ${ }^{121}$ Die einfache Lizenz verleiht dem Lizenznehmer dagegen keine Ausschlussrechte gegenüber Dritten. ${ }^{122}$ Unabhängig davon, ob es sich um eine einfache oder ausschließliche Lizenz handelt, besteht die Übereinstimmung, dass der Lizenzvertrag ein gegenseitiger Vertrag gemäß $\int S 320$ ff BGB ist. ${ }^{123}$ Er wird heute allgemein als ein Vertrag sui generis angesehen. ${ }^{124}$

\footnotetext{
113 Henn, Rn. 37. S. 28.; Haver/Mailänder, S. 15 f.;

114 Weinmann, S. 3.; Vgl. Ulmer-Eilfort/Schmoll, S. 3.

115 Vgl. Zum Lizenzvertrag siehe dazu Langen, Eugen, Internationale Lizenzverträge, S. 11 ff.; Henn, Problematik S. 25 ff.; Gaul/Bartenbach, S. K 13 ff.; Troller, Immaterialgüterrecht II, S. 843 f.; Hoppe, S. 45 ff.; Tetzner, Leitfaden, S. 108 ff.; Weinmann, S. 10 ff.; Byrne, S. 99 ff.; Pedrazzini, Patentrecht, S. 125 ff.

116 Emmerich, S. 412.; Siehe dazu, Byrne, Noel, Licensing Technology, S. 215. „, technology licensing agreement is usually more than simply a licence for a money consideration called a running royalty, to do what otherwise the licensor could have prevented; it is more than mere protection against infringement proceedings. It is a contract in which the licensor undertakes to put the licensee in possession of Know-how and other information, if not also of the physical means, necessary to make a product or provide a service.”

117 Siehe dazu zur Rechtsnatur des Lizenzvertrages, Benkard, § 15 Rn. 81 f. S. 578 f.; Rasch, S. 119.; Gitter, S. 396 ff.; Lüdecke/Fischer, S. 32 f.; Hasselblatt, § 38 Grundzüge des Lizenzvertrages, Rn. 3/4. S. 1456.; Gaul/Bartenbach, Rn. 8 ff.; Stumpf/Groß, S. 42 ff.; Henn, S. 28 ff.; Pfaff/Osterrieth, Rn. 33 ff. S. 8 ff.; Widmer, S. 25 ff.; Troller, Immaterialgüterrecht II, S. 821.; Troller, GRUR 1952, S. 108 ff.; Blum/Pedrazzini II Art. 34. Anm. 42 ff.; Pedrazzini, Patentrecht S. 126 ff.; Böhme, S. 38.; Haver/Mailänder, S. 33 ff.; Klauer/Möhring, § 9 Rn. 24. S. 457 ff.; Reimer, § 9 Rn. 5. S. 461.; Tetzner, § 9 Rn. 7. S. 694.; Lindenmaier/Weiss, § 9 Rn. 28 S. 347 f.; Altin-Sieber, S. 117 ff.; Knap, GRURInt. 1973, S. 226 f.; Wang, Jing-An, S. 27 ff.

${ }^{118}$ Henn, Rn. 38. S. 28.

119 Gaul/Bartenbach, Rn. 370, S. K 136.; Vgl. Busse, Patentgesetz, § 15 Rn. 57. S. 401.

120 Vgl. dazu Stumpf/Groß, Rn. 13. S. 35.; Henn, Rn. 16. S. 11.

${ }^{121}$ Ulmer-Eilfort/Schmoll, S. 3; Vgl. Osterrieth, Patentrecht, Rn. 324 f. S. 178.

122 Ulmer-Eilfort/Schmoll, S. 3.

123 Nirk, GRUR 1970 S. 329 f.; Osterrieth, Patentrecht, Rn. 297. S. 157.

124 Vgl. BGHZ 2, S. 331, 335; 26, S. 7, 9 - Sympatol III; BGH GRUR 1961, S. 27, 29 - Holzbauträger; GRUR 1970, S. 547 f. - Kleinfilter; Benkard, § 15, Rn. 81. S. 578.; Troller, Immaterialgüterrecht II, S. 821 ff.; Busse, Patentgesetz, § 15 Rn. 53. S. 400 f.; Osterrieth, Patentrecht, Rn. 297. S. 157.; Stumpf/Groß, Rn. 19 ff. S. 42 ff.; Henn. Rn. 118. S. 72 f. „Die Rechtsprechung hat seit dem Maffei-Urteil des RG vom 11. 11. 1933 uneingeschränkt die sogenannte sui-generis-Theorie vertreten.“; RGZ 142, 212, 213, „Der Vertrag über die Ausnutzung eines Patents (Lizenzvertrag) begründet ein Rechtsverhältnis eigener Art, das nach seinem besonderen wirtschaftlichen Inhalt sehr mannigfaltig sein, auch rechtlich eine gemischte Natur aufweisen kann.“
} 


\section{Die Arten des Lizenzvertrages}

\section{Ausschließliche Lizenz}

Die h. M. betrachtet die Einräumung einer ausschließlichen Lizenz als einen Akt der Rechtsübertragung. ${ }^{125}$ Im Unterschied zur einfachen Lizenz gewährt die ausschließliche Lizenz dem Lizenznehmer das alleinige Recht zur Verwertung der Erfindung, ggf. auch gegenüber dem Lizenzgeber selbst. ${ }^{126}$

Der Lizenzgeber verpflichtet sich, im Lizenzgebiet keine weiteren Lizenzen $\mathrm{zu}$ vergeben (Alleinlizenzklausel - sole-license clause). ${ }^{127}$ Je nach Vertragsgestaltung kann dem Lizenznehmer sogar die Ausschließlichkeit eingeräumt werden, dass sich der Lizenzgeber verpflichtet, sich jeder Eigennutzung der Erfindung im Lizenzgebiet $\mathrm{zu}$ enthalten (Alleinbenutzungsklausel - single-use clause) ${ }^{128}$.

Die ausschließliche Lizenz hat eine dem Sachenrecht angenäherte, gleichsam dingliche, absolute Natur. ${ }^{129}$ Sie wirkt gegen den Rechtsnachfolger des Lizenzgebers, wenn das Patentschutzrecht vom Lizenzgeber auf den Erwerber übergeht.

Es steht der Ausschließlichkeit der Lizenz nicht entgegen, wenn der Lizenzgeber vor der Erteilung der ausschließlichen Lizenz eine oder sogar mehrere einfache Lizenzen bestellt hat. ${ }^{130}$ Er verpflichtet sich lediglich, keine weiteren Lizenzen für dasselbe Gebiet zu erteilen und dem Lizenznehmer in diesem Gebiet selbst keine Konkurrenz zu machen. ${ }^{131}$

\section{Alleinige Lizenz (,,sole licence ${ }^{6}$ )}

Es ist von einer alleinigen Lizenz ${ }^{132}$ die Rede, wenn zwar nur einem einzigen Lizenznehmer für ein gewisses Gebiet eine Lizenz gegeben wird, der Lizenzgeber selbst jedoch sein Nutzungsrecht behält. Diese Begriffsform ist aus der anglo-amerikanischen Unterscheidung zwischen „exclusive licence“ und „sole licence" abgeleitet. ${ }^{133}$

\section{Einfache Lizenz}

Eine einfache Lizenzerteilung ist dann gegeben, wenn der Lizenznehmer keine Ausschließlichkeitsstellung besitzt. ${ }^{134}$ Bei der einfachen Lizenz kann der Lizenzgeber beliebig viele Lizenzen auf dem gleichen sachlichen und örtlichen

\footnotetext{
125 Sosnitza, S. 189.; Benkard, § 15 Rn. 89. S. 580.

${ }^{126}$ Gaul/Bartenbach, Rn. 66. S. K 28.; Vgl. Lüdecke/Fischer, S. 317.;Henn, Rn. 145. S. 91.; Guilino, S. 187.; Byrne, S. $22 \mathrm{ff}$. „The licensor who grants an exclusive licence covenants (if not expressly, then impliedly) with his licensee, first, not to license any other person to exploit the licensed property in the territory conceded to the licensee; and second, not to exploit it there himself or by his agents.“

${ }^{127}$ Vgl. Gaul/Bartenbach, Rn. 67. S. K 29.; Kortunay, S. 21.

128 Vgl. Gaul/Bartenbach, Rn. 67. S. K 29.; Benkard, § 15 Rn. 97. S. 581.; Stumpf/Groß, Rn. 36. S. 54.; Lüdecke/Fischer, Rn. D 12. S. 367.; Hoppe, S. 47 f.; Reimer, § 9, Rn. 6. S. 462.; Kortunay, S. 21.

${ }^{129}$ RGZ 57, 38, 40 f.; 130, 275, 282; 134, 91, 96; Forkel, S. 75, 78; Benkard, § 15 Rn. 92.S. 580.; Kortunay, S. 22.; Lüdecke/Fischer, Rn. D 12. S. 368.; Osterrieth, Patentrecht, Rn. 325. S. 178.; Klauer/Möhring, § 9 Rn. 25. S. 460.

${ }_{130}$ RG v. 17. 3. 1934, GRUR 1934, 306, 307; Reimer, § 9 Rn. 84. S. 550.

131 Vgl. Benkard, § 15 Rn. 94. S. 581.;

132 Hierzu Stumpf/Groß, Rn. 38. S. 55; Henn, Rn. 145. S. 91.; Gitter, S. 383 f.; Hoppe, S. 59.

133 Henn, Rn. 145. S. 91.; Vgl. Stumpf/Groß, Rn. 38. S. 55.; Hoppe, S. 59.

134 Gitter, S. 381.
} 
Vertragsgebiet für den gleichen Zeitraum erteilen. ${ }^{135}$ Er selbst ist auch in der Benutzung des Schutzrechts frei.

Um die Wettbewerbsfähigkeit des einfachen Lizenznehmers aufrechtzuerhalten, wird oft eine sog. Meistbegünstigungsklausel ${ }^{136}$ vereinbart. ${ }^{137}$ Sie hat den Zweck, zugunsten des erstbegünstigten Lizenznehmers sicherzustellen, dass den späteren Lizenznehmern die Lizenz nur unter den gleichen Bedingungen eingeräumt werden kann. ${ }^{138}$

Bei der einfachen Lizenz wird nur eine schuldrechtliche Beziehung zwischen den Vertragsparteien als die Rechtsgrundlage der wechselseitigen Rechte und Pflichten gebildet. ${ }^{139}$ Deswegen enthält der Inhaber einer einfachen Lizenz im Gegensatz zur ausschließlichen Lizenz nur ein gewöhnliches Benutzungsrecht. ${ }^{140}$ Deshalb kann er nicht selbständig gegen Dritte vorgehen. ${ }^{141}$ Im Grundsatz kann der einfache Lizenznehmer auch nicht vom Lizenzgeber verlangen, dass dieser Verletzungen des lizenzierten Schutzrechts durch Dritte verfolgt. ${ }^{142}$ Ein Ausgleich wird dann im Regelfall über die sog. Meistbegünstigungsklausel gefunden. Bei Nichtverfolgung kann der Lizenznehmer eine Minderung der Lizenzgebühren oder gar ihren Wegfall in Anspruch nehmen. ${ }^{143}$

\section{Bezirks- und Gebietslizenz}

Wie sich aus $\int 15$ Abs. 2 Satz 1 PatG ergibt, ist es rechtlich zulässig, bezogen auf den Geltungsbereich eines nationalen Schutzrechts den Nutzungsbereich zu Lasten des Lizenznehmers zu beschränken. ${ }^{144}$ Die Beschränkung kann bei Schutzrechtslizenzen in der Weise geschehen, dass die Lizenz nur für einen bestimmten Teil des Gebiets, für das das Schutzrecht besteht, erteilt wird. ${ }^{145}$ Der durch eine Gebietslizenz gebundene Lizenznehmer hat Nutzungshandlungen außerhalb des lizenzierten Gebietes zu unterlassen. ${ }^{146}$ Der Sinn einer Gebietslizenz geht dahin, den Lizenznehmer auf Nutzungshandlungen in diesem Vertragsgebiet zu beschränken und ihn vom Wettbewerb außerhalb dieses Bezirkes fernzuhalten. ${ }^{147}$

135 Henn, Rn. 147. S. 92.; Vgl. Gaul/Bartenbach, Rn. 78. S. K 35.

136 Siehe dazu, Pagenberg/Geissler, Rn. 56. S. 258 ff.; Klauer/Möhring, § 9 Rn. 45. S. 473.; Tetzner, § 9 Rn. 44 . S. 730.

${ }^{137}$ Gitter, S. 381.

138 Vgl. Gaul/Bartenbach, Rn. 85. S. K 38.; Gitter, S. 381.

139 Vgl. Stumpf/Groß, S. 56.; Gaul/Bartenbach, Rn. 78. S. K 35.

140 BGH v. 23. 03. 1982. GRUR 1982 Heft 07. S. 411 ff. - Verankerungsteil, „1. Eine an einem Patent erteilte einfache Nutzungserlaubnis ist im Regelfalle schuldrechtlicher Natur. 2. Eine von dem Veräußerer eines Patents erteilte einfache Nutzungserlaubnis mit schuldrechtlicher Wirkung verpflichtet den Erwerber des Patents nicht..." ; Vgl. Stumpf/Groß, Rn. 39. S. 56.;Lüdecke/Fischer, S. 370.; Kortunay, S.23.; sehr kritisch, Forkel, S. 78 ff.; Forkel, NJW 1983, S. 1764 ff.; Völp, GRUR 1983, S. 45 ff.; Mager, GRUR 1983, S. 51 ff.; Brandi-Dohrn, GRUR 1983, S. 146 ff.; Rosenberger, GRUR 1983, S. 203 ff.; Osterrieth, Patentrecht, Rn. 329. S. 180.; Klauer/Möhring, § 9 Rn. 42. S. 472.

${ }^{141}$ RGZ 83, 93, 95.

142 Vgl. KG v. 25.10.1939, GRUR 1940, S. 32, 33.; Gaul/Bartenbach, Rn. 84. S. K 37.

143 Vgl. Gaul/Bartenbach, Rn. 84. S. K 37.

144 Vgl. Gaul/Bartenbach, Rn. 128. S. K 53.; Benkard, § 15 Rn. 66. S. 574.

145 Stumpf/Groß, Rn. 182. S. 125.

146 Benkard, § 15 Rn. 66. S. 574.

147 Gaul/Bartenbach, Rn. 128. S. K 53. 


\section{Zeitlizenz}

Die Dauer der Lizenz richtet sich nach dem vereinbarten Vertrag. Mangels besonderer Abreden oder Umstände ist eine Lizenz zeitlich unbeschränkt, d.h. für die ganze Laufzeit des Schutzrechts erteilt. ${ }^{148}$ Die Parteien eines Lizenzvertrages können die Lizenz auf einen bestimmten Abschnitt der Laufzeit des Patents beschränken. ${ }^{149}$ Nach Ablauf der Lizenz darf der Lizenznehmer den Gegenstand des Schutzrechts nicht mehr herstellen oder vertreiben. Bei der Lizenzierung mehrerer Schutzrechte ist eine sog. Längstlaufklausel üblich. In diesem Fall endet der Vertrag erst bei Erlöschen des letzten lizenzierten Schutzrechts. ${ }^{150}$

\section{Betriebslizenz (Unternehmenslizenz)}

Bei der Betriebslizenz wird die Lizenz für die Produktion in einem bestimmten Betrieb oder Betriebsteil erteilt. ${ }^{151}$ Daher darf sie nur im Rahmen des Betriebs ausgeübt. Hierunter wird in der Regel nicht eine Fabrikanlage zu verstehen sein, sondern der wirtschaftliche Komplex des Unternehmens. ${ }^{152}$ Sie ist sowohl als ausschließliche wie als einfache Lizenz möglich und zulässig. ${ }^{153}$ Allerdings ist sie ihrem Wesen nach vorrangig auf die einfache Lizenz bezogen. Die einfache Lizenz ist grundsätzlich nicht übertragbar; sie ist als schuldrechtliche Erlaubnis zur Benutzung der Erfindung personen- und betriebsgebunden. ${ }^{154}$ Deshalb ist die Betriebslizenz grundsätzlich nicht übertragbar. ${ }^{155}$ Eine übertragbare Betriebslizenz kann nur mit dem Betrieb übertragen werden, eine Spaltung der Lizenz ist nicht zulässig, aber die Lizenz kann mit einem selbständigen Betriebsteil übertragen werden. ${ }^{156}$ Eine Betriebslizenz erlischt mit der endgültigen Aufgabe oder Einstellung des Gewerbe- oder Geschäftsbetriebes. ${ }^{157}$ An einer Betriebslizenz können im Zweifel keine Unterlizenzen vergeben werden. ${ }^{158}$

\section{Persönliche Lizenz}

Die Lizenz kann zunächst dem Lizenznehmer persönlich erteilt werden. Die persönliche Lizenz ist an die Person des Lizenznehmers gebunden und unübertragbar, d. h. unvererblich und unveräußerlich. ${ }^{159}$ Insofern bleibt er immer noch aus dem Vertrag berechtigt, wenn er seinen bisherigen Betrieb einstellt und die Lizenz in einem neuen fortführen.

\section{Konzernlizenz}

\footnotetext{
${ }^{148}$ Lüdecke/Fischer, S. 395.; Vgl. Gaul/Bartenbach, Rn. 133. S. K 62.; RG v. 9. 9. 1936, GRUR 1937, S. 1003, 1005.

149 Benkard, § 15 Rn. 65. S. 574.; Gaul/Bartenbach, Rn. 135. S. K 63.

150 Vgl. Gaul/Barten, Rn. 141. S. K 66; Kortunay, S. 24 f.

151 Vgl. Stumpf/Groß, Rn. 41. S. 57.; Henn, Rn. 171, 172. S. 107.; Gaul/Bartenbach, Rn. 150. S. K 72.; Benkard, § 15 Rn. 70. S. 575.

152 Stumpf/Groß, Rn. 41, S. 57; Vgl. Lüdecke/Fischer, S. 399.

153 Vgl. Lüdecke/Fischer, S. 395.

154 BGHZ 62, 272, 274 - Anlagengeschäft.

155 RGZ 134, 91, 97.

156 RG GRUR 30, S. $174 \mathrm{f}$.

${ }_{157}$ RG GRUR 39, S. 963, 964; Vgl. Benkard, § 15 Rn. 70. S. 576.; Henn, S. 108.; Gaul/Bartenbach, Rn. 151. S. K 73

158 Vgl. Benkard, § 15 Rn. 70. S. 575.; Henn, S. 108.; Gaul/Bartenbach, Rn. 151. S. K 73.

159 Vgl. Benkard, § 15 Rn. 70. S. 575.; Stumpf/Groß, Rn. 40. S. 56.; Gaul/Bartenbach, Rn. 152. S. K 73 f.
} 
Eine Konzernlizenz ist eine besondere Form der Unterlizenz, bei der die konzerngemäß mit dem Lizenznehmer verbundenen Unternehmen über diesen lizenzgemäß berechtigt und verpflichtet sind. ${ }^{160}$ Die Konzernlizenz erweitert den Kreis der Nutzungsberechtigten auf alle mit dem Lizenznehmer konzerngemäß verbundenen Gesellschaften. ${ }^{161}$ Es ist empfehlenswert, die mögliche Mitbenutzung durch weitere Konzernfirmen von vornherein in den Vertrag einzubeziehen und die in Betracht kommenden Konzernunternehmen im Einzelnen klarzustellen.

Konzernlizenzen spielen in Staatshandelsländern eine große Rolle. Zu Recht weist Henn ${ }^{162}$ darauf hin, dass es in Ländern mit zentral gelenkter Wirtschaft und mit staatlich gelenkten Betrieben und Verwaltungen wichtig ist, das wirklich entscheidende Unternehmen bzw. die steuernde Verwaltung als Lizenzpartner zu verpflichten und die anderen untergeordneten Betriebe mit einzuschließen, da andernfalls eine genaue Kontrolle über die Nutzung der Lizenz schwierig, wenn nicht unmöglich ist. ${ }^{163}$

\section{Quotenlizenz}

Die mengenmäßige Beschränkung der Produktion des patentgeschützten Gegenstandes auf eine Höchst- oder Mindestmenge ist eine Konkretisierung des Nutzungsrechts und der Ausübungspflicht des Lizenznehmers. ${ }^{164}$ Das ist z. B. der Fall bei der Beschränkung der Herstellung monatlicher bestimmter Produktionsmengen. Falls der Lizenznehmer die ihm gesetzte Quote überschreitet, stellt dies eine Vertragsverletzung dar. Kartellrechtlich gesehen, sind derartige Quotenlizenzen im Rahmen der Ausübungspflicht des Lizenznehmers zulässig. ${ }^{165}$

10. Herstellungslizenz

Die Herstellungslizenz 166 gestattet dem Lizenznehmer lediglich die gewerbsmäßige Herstellung des lizenzierten Gegenstandes. ${ }^{167}$ Von einer reinen Herstellungslizenz ist dann auszugehen, wenn der Lizenznehmer zur ausschließlichen Lieferung an den Lizenzgeber verpflichtet ist. ${ }^{168}$

Fehlt es an einer ausdrücklichen Regelung bezüglich der Abnahme, so schließt im Zweifel die Einräumung einer Herstellungslizenz das Recht des Lizenznehmers ein, das Erzeugnis auch in Verkehr zu bringen und zu gebrauchen. ${ }^{169}$

Ein Sonderfall der Herstellungslizenz ist die Entwicklungslizenz. Dabei wird dem Lizenznehmer das Recht eingeräumt, den Gegenstand der Lizenz selbst auf

\footnotetext{
${ }^{160}$ Henn, Rn. 171. S. 107.

161 Benkard, § 15 Rn. 70. S. 576.; Gaul/Bartenbach, Rn. 153. S. K 74.

${ }^{162}$ Henn, Rn. 178, S. 111.

163 Vgl. Stumpf/Groß, Rn. 42, S. 58.; Gaul/Bartenbach, Rn. 154. S. K 74.; Hoppe, S. 56.

164 Benkard, § 15 Rn. 71. S. 576.

165 Gaul/Bartenbach, Rn. 155. S. K 75.

166 RGZ 64, S. 143 f.; BGH GRUR 59, S. 528, 531. - Autodachzelt; 66, S. 576, 578, 580. - Zimcofot

167 Gaul/Bartenbach, Rn. 159. S. K 76.

168 Vgl. Benkard, § 15 Rn. 69. S. 575.

169 Herrsche Meinung, Vgl. RG, 26. 2. 1916, GRUR 1916, S. 178; Stumpf/Groß, Rn. 33. S. 50.; Gaul/Bartenbach, Rn. 159. S. K 77.; Lüdecke/Fischer, Rn. D 52. S. 406 ff.
} 
eigene Kosten weiter zu entwickeln. ${ }^{170}$ Der Anlass des Lizenzgebers ist meistens so, dass er die hohen Entwicklungskosten nicht allein tragen kann und das Risiko mit einem potenten Lizenznehmer zu teilen bevorzugt.

11. Vertriebslizenz

Die Verkaufs-, Handels- oder Vertriebslizenz gestattet dem Lizenznehmer, den lizenzierten Gegenstand lediglich zu vertreiben, d. h. ihn feilzuhalten und in den Verkehr zu bringen. ${ }^{171}$ Die Herstellung ist ausschließlich dem Lizenzgeber oder einem von ihm eingeschalteten weiteren Unternehmer vorbehalten, wobei die Auslieferung direkt ab Lager des Lizenzgebers erfolgt. Sobald eine eigene Auslieferung durch den Lizenzgeber erfolgt, wird sie patentfrei, wenn die Ware in den Verkehr gelangt. ${ }^{172}$

Herstellungs- und Vertriebsrecht können gebunden ${ }^{173}$ oder getrennt ${ }^{174}$ sein. In der Regel wird eine Lizenz sowohl für die Herstellung als auch für den Vertrieb der Ware erteilt. ${ }^{175}$ Liegt keine Sonderabrede über den Umfang der lizenzierten Befugnisse vor, so ist anzunehmen, dass Herstellung und Vertrieb gestattet sind. ${ }^{176}$

Besondere Arten der Vertriebslizenz stellen die Ausfuhr- und die Einfuhrlizenz dar. Eine Ausfuhrlizenz ist dann notwendig, wenn der Lizenzgeber in den Ländern, in die exportiert werden soll, Patentschutz genießt. ${ }^{177}$ Ohne seine Zustimmung kann eine im Inland hergestellte Ware nicht ins Ausland exportiert werden. Es bedarf ebenfalls einer Einfuhrlizenz, wenn ein Schutzrecht für das Importgut im Inland vorliegt. ${ }^{178}$

\section{Gebrauchslizenz}

Bei der Gebrauchslizenz ist dem Lizenznehmer nur die Benutzung oder der Gebrauch der Ware erlaubt, die meistens miet- oder pachtweise überlassen wird. ${ }^{179}$ Wird dem Lizenznehmer eine große Anlage zu Produktionszwecken zur Verfügung gestellt, dann wird diese Anlage mit der Eigentumsübertragung patentfrei, wenn er die Anlage vom Lizenzgeber erwirbt. Dies gilt für Sachpatente in gleicher Weise wie für Produkte, die nach einem Verfahrenspatent hergestellt sind. ${ }^{180}$

13. Unterlizenz (Sublizenz)

Bei der Unterlizenz handelt es sich um eine von der Hauptlizenz abgeleitete Lizenz. ${ }^{181}$ Der Lizenznehmer kann Unterlizenzen mit oder ohne besondere

${ }^{170}$ Vgl. Gaul/Bartenbach, Rn. 160. S. K 77.; Stumpf/Groß, Rn. 33. S. 52.

171 Gaul/Bartenbach, Rn. 161. S. K 77.; Vgl. Lüdecke/Fischer, Rn. D 53. S. 410.

172 Stumpf/Groß, Rn. 29. S. 48.; Gaul/Bartenbach, Rn. 161. S. K 77.

173 RG GRUR 39, S. 374.

174 RG MuW 36, S. 14 f.

175 Stumpf/Groß, Rn. 26. S. 45.

176 Lüdecke/Fischer, Rn. D 52. S. 407.; Stumpf/Groß, Rn. 26. S. 46.

177 Gaul/Bartenbach, Rn. 162. S. K 78.; Vgl. Lüdecke/Fischer, Rn. D 55. S. 417.; Stumpf/Groß, Rn. 30. S. 48.

178 Vgl. Stumpf/Groß, Rn. 30. S. 48.; Lüdecke/Fischer, Rn. D 55. S. 416.; Gaul/Bartenbach, Rn. 162. S. K 79.

179 RG v. 11. 5. 1929, RGZ 124, S. 317.; Vgl. Benkard, § 15 Rn. 69. S. 575.; Stumpf/Groß, Rn. 34. S. 52.;

Gaul/Bartenbach, Rn. 164. S. K 79.

180 BGH v. 24. 9. 1979, GRUR 1980, S. 38. Gaul/Bartenbach, Rn. 164. S. K 79.; Stumpf/Groß, Rn. 34. S. 52.

181 Gaul/Bartenbach, Rn. 193. S. K 87.; Henn, Rn. 165. S. 101.; Stumpf/Groß, Rn. 233.; Lüdecke/Fischer, Rn. D 
Zustimmung des Hauptlizenzgebers erteilen, da die Einräumung einer ausschließlichen Lizenz das Recht beinhaltet, Unterlizenzen vergeben zu dürfen. ${ }^{182}$ Bei der einfachen Lizenz ist dagegen die Zustimmung des Lizenzgebers zum Abschluss des Unterlizenzvertrages im Zweifel erforderlich. ${ }^{183}$

Hauptmerkmal für diesen wichtigen Vertragstyp ist demnach die Abhängigkeit. ${ }^{184}$ Falls die Hauptlizenz unwirksam ist, tritt die Unterlizenz auch außer Kraft. Diese Abhängigkeit zeigt sich natürlich nicht allein in der Dauer und Rechtsgültigkeit der Verträge, sondern auch und vornehmlich im Lizenzumfang. ${ }^{185}$

\section{F. Know-how-Vertrag}

\section{Einführung}

Neben dem Patentlizenzvertrag gewinnt der Know-how-Vertrag ${ }^{186}$ - häufig auch als „Technical Cooperation Agreement“ oder „Technical Assistance Agreement" bezeichnet - immer mehr an Bedeutung. Der Know-how-Vertrag ist ein gegenseitiger Vertrag, wodurch der Know-how-Geber zur Übergabe des Vertragsgegenstandes an den Know-how-Nehmer gegen eine Vergütung verpflichtet ist. ${ }^{187}$ Gegenstand eines lediglich auf die Übermittelung technischen Erfahrungswissens gerichteten reinen Know-how-Vertrages ist es, dem Vertragspartner das vertragsgegenständliche Erfahrungswissen technischer Art zur Verfügung zu stellen. ${ }^{188}$ Auch Verträge über zum Patent angemeldete Erfindungen, die noch nicht offen gelegt oder bekannt gemacht wurden, sind als Know-how-Verträge zu werten. ${ }^{189}$ Der Know-how-Vertrag kann formfrei abgeschlossen werden. ${ }^{190}$

\section{Rechtsnatur des Know-how-Vertrages}

Da auch Streit über die Rechtsnatur des Know-how ${ }^{191}$ herrscht, ist es nicht mehr verwunderlich, dass man bei der Einordnung des Know-how-Vertrages in das System der bürgerlich-rechtlichen Vertragstypen zu unterschiedlichen Ansätzen

61. S. 425.; Gitter, S. 384.; Kortunay, S. 25.; Hoppe, S. 58.

182 BGH v. 7. 11. 1952, GRUR 1953, 114, 118 - Reinigungsverfahren; BGH v. 26. 11. 1954, GRUR 1955, 338,

340 - Beschlagfreie Brillengläser; Stumpf/Groß, Rn. 233. S. 142.; Pagenberg/Geissler, S. 108 ff.; Lüdecke/Fischer, S. 430 f.; Reimer, § 9 Rn. 85. S. 550 f.; Benkard, § 15 Rn. 105. S. 583.; Osterrieth, Patentrecht, Rn. 325. S. 178.; Tetzner, § 9 Rn. 55. S. 738.; Busse, Patentgesetz, § 19 Rn. 75. S. 405.

${ }^{183}$ Vgl. Henn, Rn. 168. S. 104.; Benkard, § 15 Rn. 105. S. 583.; Stumpf/Groß, Rn. 233. S. 142.; Klauer/Möhring, § 9 Rn. 44. S. 473.; Tetzner, § 9 Rn. 42. S. 729.

${ }^{184}$ Henn, Rn. 166. S. 102.

185 Henn, Rn. 166. S. 102.

186 Siehe dazu zum Know-how-Vertrag, Pagenberg/Geissler, S. 200 ff.; Pagenberg, Lizenzverträge, S. 59.; Gaul/Bartenbach, Rn. 19 ff. S. Q 9 ff.; Troller, Immaterialgüterrecht II, S. 844 ff. Stumpf, Der Know-how-Vertrag, S. 19 ff.; Henn, S. 22 ff.; Pfaff/Osterrieth, Rn. 380 ff. S. 241 ff.; Hoppe, S. 68 ff.; Böhme, S. 30 ff.; Stumpf, S. 19 ff. $156 \mathrm{ff}$.

${ }^{187}$ Fischer, Friedrich B., Grundzüge des Gewerblichen Rechtsschutzes, S. 253 f.; Vgl. Hoppe, S. 69.; Gitter, S. 438 f.; Altin-Sieber, S. 119 ff.

${ }^{188}$ Vgl. Gaul/Bartenbach, S. Q 9.; Benkard, § 15 Rn. 232. S. 614. „Gegenstand eines Veräußerungs- oder

Lizenzvertrages können auch nicht geschützte Erfindungsleistungen, Fabrikationsverfahren, Konstruktionen oder sonstige die Technik bereichernde Leistungen sein.“

189 Stumpf, Der Know-how-Vertrag, S. 27.

190 Gitter, S. 449. Vgl. Benkard, § 15 Rn. 234. S. 615.

191 Siehe oben Erster Teil, B VI 2. 
gekommen ist. ${ }^{192}$

Knoppe 193 und Böhme 194 sind der Ansicht, der Inhalt eines Know-how-Vertrages sei so vielgestaltig, dass man keine festen Regeln aufstellen könne, welche Vorschriften des Gesetzes auf den Know-how-Vertrag passten. ${ }^{195}$ Dieser sei vielmehr ein Vertrag eigener Art. ${ }^{196}$ Es können im Wesentlichen die Regeln über die Rechtspacht entsprechend angewandt werden. ${ }^{197}$

Lüdecke/Fischer ${ }^{198}$ sind dagegen der Auffassung, ein solcher Vertrag stehe generell dem Kaufvertrag nahe. ${ }^{199}$ Deshalb seien kaufvertragliche Vorschriften auf ihn anzuwenden.

Vereinzelt findet sich in der Literatur für einen Vertrag, der die Übermittlung von Know-how zum Gegenstand hat, die Bezeichnung Lizenzvertrag ${ }^{200}$.

Da der Inhalt des Know-how-Vertrages ganz unterschiedlich ausgestaltet werden kann, kann ein Vertrag dieser Art auch verschiedene rechtliche Elemente enthalten. Im Vordergrund stehen die Eigenschaften als Kauf-, Dienst-, Werkoder Gesellschaftsvertrag, ohne dass sich der Vertrag in der Regel unter einen gesetzlichen Typus unterordnen ließe. ${ }^{201}$

\section{Arten des Know-how-Vertrages}

1. Einseitiger und zweiseitiger Know-how-Vertrag

Bei den einseitigen Know-how-Verträgen ${ }^{202}$ erfolgt ein einseitiger Wissenstransfer gegen Entgelt. Dagegen ist bei zweiseitigen Know-how-Verträgen Vertragsgegenstand der regelmäßige Austausch des von den Vertragspartnern auf einem bestimmten Gebiet entwickelten Know-how. ${ }^{203}$ Beide Arten von Know-how-Verträgen können zeitlich beschränkt oder unbeschränkt abgeschlossen werden. ${ }^{204}$

2. Einfache und ausschließliche Know-how-Erteilung

Wie beim oben genannten Lizenzvertrag 205 ist zwischen ausschließlicher und einfacher Know-how-Erteilung zu differenzieren. Bei der ausschließlichen Know-how-Erteilung enthält der Know-how-Nehmer das ausschließliche Nutzungsrecht, während bei der einfachen Know-how-Erteilung der

\footnotetext{
192 Vgl. Gitter, S. 445.

193 Knoppe, S. $23 \mathrm{ff}$.

194 Böhme, S. 30 ff.; Skaupy, GRUR 1964, S. 539 ff.

195 Stumpf, Der Know-how-Vertrag, S. 41.

196 Vgl. Benkard, § 15 Rn. 234. S. 615.; Siehe dazu zum Know-how-Vertrag als Vertrag sui generis, Gitter, S. 448.; Böhme, S. 37. „Der Know-how-Vertrag ist ein Vertrag eigener Art, der in seinen vielfältigen Vertragsgestaltungen keinem Vertragstyp des bürgerlichen Gesetzbuches direkt angehört.“

197 Vgl. Benkard, § 15 Rn. 234. S. 615.; Pfaff, BB 1974, S. 569.; Körner, GRUR 1982, S. 341 f.

198 Lüdecke/Fischer, S. 661.

199 Stumpf, Der Know-how-Vertrag, S. 41.

200 Gericke, S. 330, 332.

201 Vgl. Stumpf, Der Know-how-Vertrag, S. 41.

${ }^{202}$ Einseitiger Know-how-Vertrag bezeichnet allein die Richtung des Wissenstransfers. Vgl. Gitter, S. 440, Fn. 14.

203 Vgl. Gitter, S. 440.; Hoppe, S. 71.

204 Vgl. Gitter, S. 440; Hoppe, S. 71.

205 Siehe oben Erster Teil, E III 2, 3.
} 
Know-how-Nehmer mit weiteren Nutzern rechnen muss. ${ }^{206}$

${ }^{206}$ Vgl. Gitter, S. 440; Hoppe, S. 71. 


\section{Zweiter Teil: Rahmenbedingungen zum}

\section{Technologietransfer in China}

\section{A. Die Interessenlage beim Technologietransfer und nationale Rechtsordnung der Entwicklungsländer}

\section{Einführung in die Problematik}

Der technische Fortschritt wird heute überwiegend als der für das wirtschaftliche Wachstum bedeutendste Faktor angesehen. ${ }^{207}$ Darauf ist der Innovationsprozess gerichtet und umfasst die gesamte Tätigkeit von der Schaffung bis zur praktischen Anwendung neuen technischen Wissens und vollzieht sich im Wesentlichen in drei Phasen: Forschung, Entwicklung und praktische Verwertung. ${ }^{208}$ Allerdings fehlt es den Entwicklungsländern an Fähigkeit zur technischen Innovation. Es bestehen zwei Möglichkeiten, um an die neue und moderne Technologie zu kommen: Entweder durch Stärkung und Förderung von technologischen Aktivitäten in den Entwicklungsländern selbst oder durch Transfer technischen Wissens aus den Industrieländern. ${ }^{209}$

Aufgrund der wirtschaftlichen Bedeutung der Technologie und der Interessenkonflikte beim Technologietransfer zwischen Staaten, Unternehmen und Individuellen bedarf es der rechtlichen Rahmenbedingungen auf internationaler und nationaler Ebene. Als politischer Leitbegriff hat die unter ihm behandelte Problematik mit der zweiten Entwicklungsdekade der dritten Welthandelskonferenz und schließlich im Zusammenhang mit der auf der sechsten Sondergeneralversammlung der Vereinten Nationen im Jahre 1974 ausgerufenen „Neuen Weltwirtschaftsordnung“ Bedeutung erlangt. ${ }^{210}$ Inzwischen wird in politischen Manifesten die Auffassung vertreten, dass Technologietransfer an Entwicklungsländer als „subtileres Medium der Beherrschung“, als eine „neue Form von Raubkapitalismus und Sklavenhandel"“ verdammt wird. ${ }^{211}$

Außer einer gerechten Weltwirtschaftsordnung auf der internationalen Ebene brauchen Entwicklungsländer wie China als Technologienehmer noch eine nationale Rechtsordnung über den Technologietransfer. Einerseits muss die Rechtssicherheit für Schutzrechte und Know-how aus den Industrieländern als Technologiegeber gewährleistet werden. Andererseits müssen

207 Kunz-Hallstein, GRURInt. 1975, S. 261.; Vgl. Hansen, IIC Vol. 11 No. 4/1980. S. 429.; Pfanner, GRURInt. 1983, S. 363.

208 Vgl. Pfanner, GRURInt. 1983, S. 362.

${ }^{209}$ Vgl. Kunz-Hallstein, GRURInt. 1975, S. 262.

210 Vgl. Stoll, S. $12 \mathrm{f}$.

${ }^{211}$ Vgl. Kunz-Hallstein, GRURInt. 1975, S. 262. 
Wettbewerbsbeschränkende Vereinbarungen und der Missbrauch der durch Technologie-Monopol verursachten marktbeherrschenden Stellung kartellrechtlich verhindert werden. ${ }^{212}$

Folgend werden verschiedene Interessen des Technologiegebers und Technologienehmers beim Technologietransfer näher erläutert, die den Grund der Gesetzgebung zum Schutz des geistigen Eigentums und den Ausgangspunkt der nationalen entwicklungsrechtlichen Gesetze und Bestimmungen darstellen.

\section{Interesse des Technologiegebers am Technologietransfer}

Sehr unterschiedlich sind die Interessen des Technologiegebers am Technologietransfer. Durch Technologietransfer auf den ausländischen Markt können sie die Gewinne maximieren, aber vorausgesetzt, dass da ein gutes Rechtssystem zum Schutz des geistigen Eigentums vorhanden ist. Die Industrieländer als Technologiegeber sehen in einem konsequenten Schutz des geistigen Eigentums eine Gewähr für die Sicherung ihrer Wettbewerbstätigkeit im Verhältnis zu den unmittelbaren Konkurrenten. ${ }^{213}$ Sie sind nicht geneigt, dieses Eigentum in Länder zu transferieren, wo sein Schutz nicht gewährleistet ist.

Dass der Technologiegeber verschiedene Arten vom Technologietransfer benutzt, kommt darauf an, welche Interessen er daran hat. So kann es sein, dass der Technologiegeber durch den Technologietransfer seinen Umsatz steigern will. ${ }^{214}$ Dies wird vor allem der Fall sein bei Lieferungen von Investitionsgütern und der Errichtung von Industrieanlagen (turn-key-contracts, Verträge über produit en main) oder dem Abschluss von Kooperationsverträgen. ${ }^{215}$

Außerdem kann der Technologiegeber wegen günstigerer Produktionsvoraussetzungen in den Entwicklungsländern durch den Abschluss von Patentlizenz- und Know-how-Verträgen sowie von Verträgen über technische Hilfe seine Produktion ins Ausland verlagern. Das Interesse des Technologiegebers am Transfer kann auch darin bestehen, dass im Ausland billige Rohstoffe vorhanden sind, deren Bearbeitung an Ort und Stelle kostengünstiger ist als am Sitz des Technologiegebers. ${ }^{216}$

\section{Interesse des Technologienehmers am Technologietransfer}

Die Entwicklungsländer sehen den internationalen Technologietransfer von den Industrieländern als den Ausweg zur Besserung ihrer sich immer mehr verschlechternden Situation im Aufbau moderner Industriebetriebe und in der Ausweitung des Exportes an. Es führt zwar zu einer negativen Lizenzbilanz eines Landes, aber damit werden enorme Forschungs- und Entwicklungsaufwendungen gespart. Diese Erfahrung konnten die Japaner machen, die früher häufig nachbauten, aber danach feststellen mussten, dass das Nachbauen teilweise viel kostspieliger ist als der Erwerb neuer Technologie, sei es durch Kauf oder durch

\footnotetext{
${ }^{212}$ Siehe dazu zur Verhinderung von Missbrauch durch gewerbliche Schutzrechte, Heath, GRURInt. 1996, S. 1179 f.

${ }^{213}$ Vgl. Schmidt-Diemitz, GRURInt. 1988, S. 291.; Täger, GRURInt. 1982, S. 64 ff.

214 Stumpf, GRURInt. 1977. S. 441.

215 Stumpf, GRURInt. 1977. S. 441.

216 Stumpf, GRURInt. 1977. S. 441.
} 
Lizenzen. ${ }^{217}$ In diesem Zusammenhang sei auch darauf hingewiesen, dass die Bundesrepublik Deutschland seit Kriegsende eine negative Lizenzbilanz aufweist. $^{218}$

Die Erkenntnisse der politischen Diskussionen und der ökonomischen und sozialwissenschaftlichen Analysen gehen übereinstimmend dahin, dringend benötigtes Kapital in diesen Ländern zu investieren, um die Infrastruktur zu verbessern, neue Arbeitsplätze zu schaffen und die Produktivität der Bevölkerung zu erhöhen. ${ }^{219}$ Objektiv betrachtet geht das Interesse der Entwicklungsländer dahin, eine Technologie zu erwerben, die auf die individuellen Bedürfnisse des jeweiligen Landes zugeschnitten ist. ${ }^{220}$ Denn der primäre Zweck der Industrialisierung liegt in der Schaffung von Arbeitsplätzen und in der Lösung des Problems der Arbeitslosigkeit. ${ }^{221}$ Deshalb hat eine arbeitsintensive Technologie im Allgemeinen den Vorrang, z. B. in China mit erheblicher Bevölkerungsdichte.

Die Interessen des Technologienehmers am Technologietransfer beruhen ebenfalls auf den unterschiedlichen Gründen. Mit Hilfe neuer Technologien kann der Technologienehmer ganz neue Produktionen aufziehen, um vorhandene Ressourcen an Arbeitskräften und Rohstoffen auszunutzen. Sehr häufig sind jedoch die Fälle, in denen eine Produktion bereits besteht, diese aber technologisch rückständig ist. Der Erwerb von Technologie dient dazu, die Produktion zu ergänzen, zu verbessern oder zu erweitern. ${ }^{222}$ Außerdem erspart der Erwerb der Technologie für ein international bereits bekanntes und begehrtes Produkt dem Technologienehmer das Risiko von Fehlentwicklungen und von Mängeln und zwar erleichtert den Absatz des neuen Produkts. ${ }^{223}$

\section{Die Rolle des Patentwesens für den Technologietransfer an Entwicklungsländer}

Die Entwicklungsländer messen nun dem Patentrecht im Rahmen dieses Transferprozesses eine sehr wichtige Rolle zu und versuchen, insbesondere durch patentrechtlich wirksame Maßnahmen den Transfer von technischem Wissen aus den Industriestaaten zu fördern und zu verbessern. ${ }^{224}$ Der gewerbliche Schutz, insbesondere der Patentschutz, nimmt wegen seiner Bedeutung für die Innovation und als Sicherungsinstrument für ausländische Investoren in den Entwicklungsländern eine besondere Rolle im Prozess der Industrialisierung ein. ${ }^{225}$

Trotz der allgemein anerkannten Rolle des Patentwesens für die Förderung des technischen und wirtschaftlichen Fortschritts ${ }^{226}$ kommt ihm in Wirklichkeit

\footnotetext{
217 Vgl. Stumpf, GRURInt. 1977, S. 442.

218 Stumpf, GRURInt. 1977, S. 442.

219 Mangalo, GRURInt. 1977, S. 350;; Vgl. Hansen, Vol. 11. No. 4/1980, S. 429 ff.

220 Schmidt-Diemitz, GRURInt. 1988, S. 291

221 Mangalo, GRURInt. 1977, S. 352.

222 Stumpf, GRURInt. 1977, S. 442.

223 Vgl. Stumpf, GRURInt. 1977, S. 442.

224 Kunz-Hallstein, GRURInt. 1975, S. 263.

225 Mangalo, GRURInt. 1977, S. 352.; Siehe dazu zur Wirkung des Patentschutzes in den Entwicklungsländern, Imam, IIC 2006, S. 245 ff.; Hilpert, GRURInt. 1998, S. 94 ff.; Zur Ausgestaltung des Systems gewerblicher Schutzrechte im nationalen Interesse, Heath, GRURInt. 1996, S. 1176 ff.

${ }^{226}$ Vgl. Machlup, GRURAusl. 1961, S. 473.; Beier, GRURInt. 1970, S. 1 f.
} 
im Rahmen des Technologietransfers nur eine beschränkte Bedeutung zu. ${ }^{227}$ Hiance und Plasseraud haben in ihrem 1972 erschienenen Buch „Brevets et sous-développement", der bisher wohl besten und sorgfältigsten Untersuchung zum Problemkreis Patentrecht und Entwicklungsländer, die These von der beschränkten Rolle, die das Patentrecht im Rahmen des Technologietransferprozesses spielen kann, zu quantifizieren versucht. ${ }^{228}$ Sie schätzen den Anteil der Patente am Prozess des Technologietransfers auf etwa zwei Prozent. 229 In Bezug auf Entwicklungsländer kann dieser Anteil wegen der geringeren technologischen Entwicklung noch niedriger liegen.

Aber die Entwicklungsländer schreiben selbst dem gewerblichen Rechtsschutz trotzdem erhebliche Bedeutung zu. In dem Umfangreichen Katalog entwicklungspolitischer Maßnahmen der Empfängerländer, der von Maßnahmen auf dem Gebiete des Niederlassungs-, Devisen- und Steuerrechts, Arbeits- und Gesellschaftsrechts reicht, fehlen in keinem Fall die gewerblichen Schutzrechte. ${ }^{230}$

In der Tat hat das Patentrecht in den Entwicklungsländern viele Vorteile. Der Erfinder hat eine technische Erfindung in der Patentschrift in einer Art und Weise zu offenbaren, dass die in der Erfindung verkörperte neue technische Lehre für den Fachmann technisch nachvollziehbar ist. Soweit Patentschutz durch Zeitablauf erloschen ist oder durch Nichtzahlung von Jahresgebühren aufgegeben wurde, steht die Gesamtheit des in offen gelegten Patentanmeldungen und Patentschriften offenbarten technologischen Wissens jedermann zur Nutzung zur Verfügung. ${ }^{231}$ Der Wert eines Patents für den Technologietransfer liegt indessen weniger in der Bedeutung des Patents als Offenbarung einer technischen Lehre für die Allgemeinheit als vielmehr darin, dass das Patent eine Rechtsposition schafft, die übertragen werden kann, oder an der Nutzungsrechte eingeräumt werden können. ${ }^{232}$

\section{Nationale Rechtsordnung der Entwicklungsländer beim Technologietransfer}

1. Das nationale Entwicklungsrecht

Neben den allgemeinen und traditionellen politischen Instrumenten zur Förderung und Kontrolle des Außenwirtschaftsverkehrs wie z. B. Technologieimportbeschränkungen haben die Entwicklungsländer seit den sechziger Jahren zunehmend spezielle Technologietransfergesetze geschaffen, nachdem sie erkannt hatten, dass sich ihre eigenen Interessen hinsichtlich der wirtschaftlichen Entwicklung ihrer Ländern vielfach nicht mit den Interessen der multinationalen Unternehmen der Industrieländer deckten. ${ }^{233}$ Während die meisten Industrieländer Kartellgesetze geschaffen haben, spielt das nationale

\footnotetext{
227 Mangalo, GRURInt. 1977, S. 353.; Vgl. Kunz-Hallstein, GRURInt. 1975, S. 263.; Stoll, 2005, S. 296 ff.

${ }^{228}$ Kunz-Hallstein, GRURInt. 1975, S. 263.

229 Vgl. Mangalo, GRURInt. 1977, S. 353.; Hiance/Plasseraud, S. 94 f.

230 Vgl. Beier/Kunz, GRURInt. 1972, S. 389.; Stumpf, GRURInt. 1977, S. 443.; Hilpert, GRURInt. 1998, S. 94 ff.

231 Vgl. Osterrieth, Patentrecht, S. 9.

232 Osterrieth, Patentrecht, S. 9.

${ }^{233}$ Vgl. UN, Joint Ventures, S. 81.
} 
Entwicklungsrecht in den Entwicklungsländern eine dominierende Rolle.

Mit dem Begriff „Nationales Entwicklungsrecht" sollen daher alle Regelungen der Entwicklungsländer bezeichnet werden, die die Entwicklung der nationalen Wirtschaft im Ausgleich mit den Interessen ausländischer Unternehmen sichern wollen. ${ }^{234}$ Die Länder müssen daher im Voraus mittels nationaler Entwicklungspläne bestimmen, in welchen industriellen Sektoren und in welchem Ausmaß sie ausländisches Kapital und Technologie benötigen. ${ }^{235}$

\section{Staatliche Kontrolle über Technologietransferverträge}

In vielen Entwicklungsländern bedürfen Technologietransferverträge, die ein inländisches Unternehmen mit einem ausländischen Technologiegeber abschließt, der Genehmigung durch eine staatliche Behörde. ${ }^{236}$

Hier nehme ich Entwicklungsländer Lateinamerikas als Beispiel. Verschiedene Länder Lateinamerikas haben zunächst einmal den gesamten Verkehr mit dem Ausland, soweit er die Übertragung oder die Lizenzierung von Patenten, Warenzeichen, Mustern und Know-how betrifft, staatlicher Kontrolle unterworfen. ${ }^{237}$ Jedes Lizenzabkommen wird überprüft hinsichtlich seiner wirtschaftlichen, technischen, rechtlichen, sozialen und politischen Aspekte um zu gewährleisten, dass Lizenzverträge tatsächlich den Entwicklungsprozess fördern. ${ }^{238}$ Die Zulässigkeit der Zahlung von Lizenzgebühren ist auf bestimmte Höchstsätze beschränkt und Wettbewerbsbeschränkende Klauseln in Lizenzabkommen sind verboten. Die einzelnen Gesetze enthalten ferner grundsätzliche Voraussetzungen für die zu übertragende Technologie. Die Eintragung und Genehmigung von Lizenzverträgen wird insbesondere dann versagt, wenn die betreffende Technologie ungünstige Auswirkungen auf Verbrauchergewohnheiten zeigen oder die Abhängigkeit von ausländischer Hilfe begründen kann. ${ }^{239}$ Die Ziele dieser Genehmigungsverfahren für Verträge über den Erwerb ausländischer Technologie wurden teilweise bereits angedeutet: Die staatlichen Behörden können auf diese Weise ihren wirtschafts-, devisen- und entwicklungspolitischen Grundsätzen Beachtung verschaffen und gleichzeitig die inländischen Vertragspartner vor überhöhten Zahlungsbedingungen und sonstigen, belastenden Vertragsklauseln schützen. ${ }^{240}$

3. Einschränkungen der Patentfähigkeit

Wesentlich einschneidender als die erwähnten Kontrollbestimmungen über Technologietransferverträge sind die Bestrebungen solcher Entwicklungsländer Lateinamerikas, ihren entwicklungspolitischen Vorstellungen durch Einschränkungen der Patentfähigkeit - wie z. B. die entwicklungspolitisch

\footnotetext{
${ }^{234}$ Vgl. Funke, S. 68.

235 Vgl. UN, Joint Ventures, S. $82 \mathrm{ff}$.

${ }^{236}$ Boguslawskij, S. 192.

237 Siehe dazu, Beier/Kunz, GRURInt. 1972, S. 389 f.; Aracama-Zorraquin, GRURInt. 1976, S. 55 f.

238 Aracama-Zorraquin, GRURInt. 1976, S. 55.

239 Aracama-Zorraquin, GRURInt. 1976, S. 55.

240 Beier/Kunz, GRURInt. 1972, S. 390.
} 
besonders umstrittenen pharmazeutischen Erfindungen ${ }^{241}$ - Rechnung zu tragen. ${ }^{242}$ Das Ziel ist in den Entwicklungsländern Lateinamerikas den Zugang zu den industriellen Kenntnissen und Prozessen zu verbessern und Wettbewerbsbeschränkungen auszuschließen, um auf diese Weise zu einer wirksameren Einsetzung der Erkenntnisse der Wissenschaft und der Technologie und zu ihrer raschen und effektiven industriellen Nutzbarmachung zu gelangen. ${ }^{243}$

\section{Verkürzung der Schutzfrist}

In einigen Entwicklungsländern zeichnet sich ferner eine gewisse Tendenz zur Verkürzung der Schutzfristen für Patente ab. Hier zu nennen ist das Beispiel Indiens, das in seinem neuen Patentgesetz von 1970 die normale Laufzeit von 16 auf 14 Jahre verkürzt hat und Verfahren zur Herstellung von Lebensmitteln und Pharmazeutika nur noch für die Dauer von fünf Jahren ab Erteilung bzw. sieben Jahre nach der Anmeldung des Patents schützt. ${ }^{244}$ Außerdem findet sich die verkürzte Schutzfrist auch in Kolumbien, wo die Schutzfrist für Patente acht Jahre seit Erteilung beträgt, wobei dem Patentinhaber die Möglichkeit verbleibt, eine Verlängerung der Schutzfrist um weitere vier Jahre zu beantragen, wenn er die Erfindung im Inland zumindest im vorausgehenden Jahr ausgeübt hat. ${ }^{245}$

5. Verschärfung der Zwangslizenzvorschriften

Als Einschränkung des gewerblichen Rechtsschutzes in der nationalen Gesetzgebung haben eine Reihe von Entwicklungsländern die Bestimmungen über die Ausübung der Erfindung und die Erteilung von Zwangslizenzen in ihren Patentgesetzen verschärft. In Art. 558 des am 1. Januar 1972 in Kraft getretenen neuen kolumbianischen Handelsgesetzbuches ${ }^{246}$ wurde die Frist zur Ausübung des Patents auf zwei Jahre ab Erteilung festgesetzt. Pharmazeutische Verfahren, die zum Patent angemeldet werden, müssen innerhalb einer Frist von einem Jahr vom Datum der Anmeldung gerechnet im Inland ausgeübt werden, andernfalls die Anmeldung als aufgegeben angesehen wird. ${ }^{247}$ Darüber hinaus können Patente aus entwicklungspolitischen Gründen einer besonderen Amtlizenz unterworfen werden, für deren Erteilung die Nichtausübung innerhalb bestimmter Frist keine

\footnotetext{
${ }^{241}$ Siehe dazu, Bartling, GRURInt. 1972, S. 396 ff.; Reinelt, GRURInt. 1972, S. 12 ff.; Correa, GRURInt. 1994, S. 799 ff.; Pacón, GRURInt. 1994, S. 899 f. „In Brasilien ist das Gesetz Nr. 5.772 vom 21. 12. 1971 noch in Kraft. Das Gesetz spiegelt die Richtung wider, der die brasilianische Regierung mehr als 20 Jahre bezüglich Industrie, Technologie und Außenhandel gefolgt ist. Diese Richtung beruht auf dem Wirtschaftsmodell von Importsubstitution und Schutz der einheimischen Unternehmen und Produkte. ... 1988 verhängte der damalige US-Präsident Reagan Handekssanktionen gegen Papier-, elektronische und chemische Produkte. Die Sanktionen wurden 1990 aufgehoben, als die Regierung von Collor versprach, einen Gesetzentwurf zur Verstärkung des Patentschutzes dem Parlament vorzulegen.“

${ }^{242}$ Vgl. Beier/Kunz, GRURInt. 1972, S. 390.; Reinelt, GRURInt. 1972, S. 12. „Im gegenwärtigen Zeitpunkt ermöglichen es - in der Regel mangels eines ausdrücklichen gesetzlichen Patentierungsverbots - die Gesetze folgender lateinamerikanischer Staaten, einen unmittelbaren Patentschutz für pharmazeutische Produkte zu erlangen: Costa Rica, Dominikanische Republik, Ekuador, Guatemala, Haiti, Honduras, Panama, Peru und El Salvador. In allen übrigen Staaten Lateinamerikas, außer Brasilien und mit gewissen Einschränkungen in Argentinien und Bolivien, besteht zur Zeit ein Patentschutz für Verfahren zur Herstellung von Arzneimitteln.“ ${ }^{243}$ Vgl. Aracama-Zorraquin, GRURInt. 1972, S. 139.

244 Vgl. Beier/Kunz, GRURInt. 1972, S. 391.; Vedaraman, IIC 1972, S. 46.

245 Vgl. Aracama-Zorraquin, GRURInt. 1976, S. 54.

246 Verordnung Nr. 410 vom 27. 3. 1971 (auf Grund der Ermächtigung durch Gesetz Nr. 16/1968).

247 Art. 540.
} 
zwingende Voraussetzung ist. ${ }^{248}$

\section{Zusammenfassung}

Das Problem des Technologietransfers berührt sowohl viele Bereiche des Lebens einer modernen Industriegesellschaft als auch die eines Entwicklungslandes. Die Beziehung zwischen den Industrie- und Entwicklungsländern findet seinen Ausdruck im sog. Nord-Süd-Konflikt 249. Die schwache Position der Entwicklungsländer beim Technologietransfer hat ihre Ursachen nicht nur im Patentwesen. Vielmehr fehlt es ihnen an Instrumentarien zur Kontrolle wirtschaftlicher Macht, die in den Industrieländern durch das Kartellrecht geschaffen wurden. Ebenso als Entwicklungsland hat China im Lauf seiner Gesetzgebung über den Technologietransfer die Erfahrungen zu berücksichtigen, die die anderen Entwicklungsländer bereits beim Patentwesen und nationalen Entwicklungsrecht gemacht haben.

\section{B. Die Gesetzgebung zum Technologietransfer in China}

Nach jahrelangen Verhandlungen ist die VR China mit Wirkung vom 11. Dezember 2001 der Welthandelsorganisation beigetreten. ${ }^{250}$ Nach dem Bericht von der WTO-Arbeitsgruppe über den Beitritt der VR China hat China bestätigt, dass China nur mit dem Übereinkommen über handelsbezogene Investitionsmaßnahmen und Übereinkommen über handelsbezogene Aspekte der Rechte des geistigen Eigentums vereinbarte Gesetze, Verordnungen oder Maßnahmen in Bezug auf den Technologietransfer, Herstellungsprozesse oder andere gesetzlich geschützte Kenntnisse auf Personen oder Unternehmen in China anwenden würde. ${ }^{251}$ Es stellt sich die Frage, wie der Technologietransfer in China gestaltet werden soll. Um diese Frage zu erklären, ist zuerst ein Überblick über die Rahmenbedingungen zu verschaffen.

\section{Einführung ins nationale Recht}

1. Zielsetzung und Grundprinzipien des Wirtschaftsverwaltungsrechts

Die wesentlichen Anforderungen des WTO Rechts an die Rechtsordnung Chinas lauten: Verstetigung des Rechtsstaatsprinzip und dessen kontinuierliche Weiterentwicklung in der Zivil- und Wirtschaftsordnung. ${ }^{252}$ Die Zielsetzung der Gesetzgebung Chinas ist die Rechtsordnung Chinas mit dem Welthandelsrecht in

248 Art. 560; Siehe dazu zur Zwangslizenz, Correa, GRURInt. 1994, S. 805.

249 Siehe dazu zum Nord-Süd-Konflikt, Stoll, Das internationale Recht im Nord-Süd-Verhältnis, Heidelberg 2005; Link, Der Nord-Süd-Konflikt und die Zusammenarbeit der Entwicklungsländer, Berlin 1985; Hüfner/Naumann, Neue Weltwirtschaftsordnung?: der Nord-Süd-Konflikt im UNO-System, Berlin 1980; Langhammer/Stecher, Der Nord-Süd-Konflikt: die Spielregeln der Weltwirtschaft im Brennpunkt, Würzburg 1980; Bohnet, Das Nord-Süd-Problem; Mangalo, GRURInt. 1977, S. 349 f.; Hilpert, GRURInt. 1998, S. 94

250 Siehe dazu Hilf, Meinhard/Göttsche, Götz J. Chinas Beitritt zur WTO, RIW 2003 S. 161 ff.

${ }^{251}$ Vgl. Working Party Report on Accession of the People's Republic of China, II. 6. State-owned and State-Invested Enterprises 49. http://docsonline.wto.org/DDFDocuments/t/WT/ACC/CHN49.doc, WT/ ACC/ CHN/ 49

${ }^{252}$ Huck, Rahmenbedingungen für den Technologietransfer von China nach Deutschland, 12.01.2005 S.5 
Einklang zu bringen. Allerdings soll das WTO-Recht auch den Rechtsvorrang gegenüber dem allgemeinen nationalen Recht Chinas genießen. Dem Welthandelsrecht geht es seit In-Kraft-Treten des Allgemeinen Zoll- und Handelsabkommen 1994 nicht darum, eine gleichsam minimalistische Staatskonzeption zu erzwingen- im Gegenteil. Ebenso wie das Allgemeine Zollund Handelsabkommen 1947 ist auch heute die WTO-Rechtsordnung durchgehend von der Erkenntnis geprägt, dass staatliche Eingriffe in den Wirtschaftsprozess zulässig sind und notwendig sein können. Dabei sind jedoch völkerrechtlich verbindlich zu beachtende Grenzen gezogen, die in ihrer Systematik durch das Nichtdiskriminierungsprinzip und das Prinzip offener Märkte gekennzeichnet sind. ${ }^{253}$ Solche grundlegenden Grundprinzipien sind auch im Bericht und Protokoll über Chinas Beitritt zur WTO betont worden.

a. Nichtdiskriminierungsprinzip

Das Nichtdiskriminierungsprinzip, das insbesondere in Art. I:1 und Art. III GATT konkretisiert ist, verlangt, dass ein Staat auswärtige Produkte aus verschiedenen Ländern untereinander ebenso gleich behandeln muss wie auswärtige im Verhältnis zu heimischen Produkten, ${ }^{254}$ nämlich das Meistbegünstigungsprinzip und das Inländerbehandlungsprinzip.

Kernstück des GATT-Regimes bildet der Grundsatz der Meistbegünstigung. Solange der Abbau der Handelshemmnisse noch nicht komplett ist, kommt diesem Grundsatz immer noch eine zentrale Funktion für die Verwirklichung gleicher Wettbewerbschancen zu. Meistbegünstigung bedeutet, dass Handelsvorteile (etwa in Bezug auf Zölle, Steuern oder Ein- und Ausfuhrabgaben) gegenüber allen Vertragsparteien gleichermaßen zu gewähren sind. ${ }^{255}$ Sobald also ein Staat einer anderen Vertragspartei bestimmte Vergünstigungen einräumt, müssen diese auf alle anderen Mitgliedstaaten des GATT erstreckt werden. ${ }^{256}$ Allerdings wird dieser Grundsatz durch eine Reihe von Ausnahmen durchbrochen. Insbesondere gelten die Ausnahmen zugunsten von Maßnahmen über den Schutz von Patenten, Warenzeichen und Urheberrechten sowie über die Verhinderung irreführender Praktiken gemäß Art. XX lit.d. GATT. Aber auch im Bereich des Handels mit Dienstleistungen werden bestimmte Zugeständnisse ${ }^{257}$ als Ausnahmen von Meistbegünstigung Art. II Allgemeines Übereinkommen über den Handel mit Dienstleistungen gemacht. Die Erbringung einer Dienstleistung besteht aus vier Möglichkeiten, nämlich

i. „aus dem Hoheitsgebiet eines Mitglieds in das Hoheitsgebiet eines anderen Mitglieds;

ii. im Hoheitsgebiet eines Mitglieds an den Dienstleistungsnutzer eines anderen Mitglieds;

iii. durch einen Dienstleistungserbringer eines Mitglieds mittels kommerzieller

253 Tietje, S. XII

254 Tietje, S. XII

255 Herdegen, S. 129.

${ }^{256}$ Herdegen, S. 129.

257 Siehe dazu Schedule of Specific Commitments on Services WT/ACC/CHN/49/Add.2 
Präsenz im Hoheitsgebiet eines anderen Mitglieds;

iv. durch einen Dienstleistungserbringer eines Mitglieds mittels Präsenz natürlicher Personen eines Mitglieds im Hoheitsgebiet eines anderen Mitglieds“..258

In diesen Zugeständnissen als Anlage zum Bericht über Chinas Beitritt zur WTO sind die vier oben genannten Gestaltungsmöglichkeiten beschränkt in den Bereichen von professionellen Dienstleistungen, Kommunikation, Bau und Ingenieurwesen, Vertrieb, Ausbildung, Umwelt, Finanzdienstleistungen, Tourismus, Transport.

In Sachen Verwendung des Inländerbehandlungsprinzips soll VR China die Verpflichtung eingehen, um allen Ausländern und ausländischen Unternehmen sowie Unternehmen mit ausländischem Kapital die gleiche Behandlung in Bezug auf den Einkauf der Einfuhren, für Herstellung notwendige Güter und Dienstleistungen sowie die Bedingungen, unter denen ihre Güter auf dem Inlandsmarkt und für die Ausfuhr hergestellt, vertrieben oder verkauft werden, zu geben. ${ }^{259} \mathrm{Nach}$ dem Inländerbehandlungsprinzip sollen das Wirtschaftssubjekt vor staatlicher Willkür und das Vertrauen in die Berechenbarkeit verwaltungsrechtlichen Handelns geschützt werden.

\section{b. Das Prinzip offener Märkte}

Im Kern des Prinzips offener Märkte soll eine faire Wettbewerbslage für alle Waren garantiert werden, um optimale wirtschaftliche Ergebnisse im Rahmen des marktwirtschaftlichen Prozesses zu erreichen. Damit es allerdings überhaupt zu einer Wettbewerbssituation kommen kann, muss dem auswärtigen Produkt der ungehinderte Marktzugang gewährt werden. ${ }^{260}$ Dies wird durch die fortschreitende Zollsenkung (Art. II GATT) und das Verbot mengenmäßiger Beschränkungen und sonstiger Maßnahmen an der Staats-(Zoll-)grenze (Art. XI:1 GATT) erreicht. ${ }^{261}$ Nach der in den 70er Jahren eingeführten Reform- und Öffnungspolitik besteht VR China schon in einem Übergang von früherer Planwirtschaft zu einer sozialistischen Marktwirtschaft. Der Beitritt Chinas in die WTO bedeutet die volle Integration der chinesischen Wirtschaft in die Weltwirtschaft. Der Marktzugang wird für ausländische Produkten durch Zollsenkung und das Verbot mengenmäßiger Beschränkungen zugesichert. Auch sind die handelsbezogene Investitionsmaßnahmen enthaltenden Rechtsvorschriften und Verwaltungsentscheidungen abgeschafft worden, die unvereinbar mit der Verpflichtung zur Inlanderbehandlung gemäß Art. III Abs. 4 GATT 1994 sowie der Verpflichtung zur allgemeinen Beseitigung mengenmäßiger Beschränkung gemäß Art. XI Abs. 1 GATT 1994 sind. ${ }^{262}$

\section{c. Transparenzprinzip}

\footnotetext{
258 Art. I. Abs. 2 Allgemeines Übereinkommen über den Handel mit Dienstleistungen (GATS)

259 Vgl. Working Party Report on Accession of the People's Republic of China II. 1. Non-Discrimination (including national treatment) 15. http://docsonline.wto.org/DDFDocuments/t/WT/ACC/CHN49.doc, WT/ ACC/ CHN/ 49

260 Tietje, S. XII

261 Tietje, S. XII

262 Siehe dazu Anhang von dem Übereinkommen über handelsbezogene Investitionsmaßnahmen (TRIMs).
} 
Grundlegend ist das Transparenzprinzip in Art. X Abs. 1 GATT 1994 vorgesehen, so dass jede Vertragspartei die Rechtsvorschriften und internationale handelspolitische Vereinbarungen unverzüglich zu veröffentlichen hat, die sich auf den Güterverkehr beziehen. ${ }^{263}$ Nach Art. X Abs. 2 GATT 1994 kommt eine handelsbezogene Maßnahme nur erst nach ihrer amtlichen Veröffentlichung in Kraft. Außerdem verlangt auch das Transparenzprinzip gemäß Art. III Abs. 1. GATS, dass jedes Mitglied umgehend und, von Notstandssituationen abgesehen spätestens zum Zeitpunkt ihres Inkrafttretens alle einschlägigen allgemeingültigen Maßnahmen veröffentlicht, die sich auf die Anwendung dieses Übereinkommens beziehen oder sie beeinträchtigen. Internationale Übereinkünfte, die für den Handel mit Dienstleistungen gelten oder ihn beeinträchtigen und die ein Mitglied unterzeichnet hat, sind ebenfalls zu veröffentlichen. Neben dieser Veröffentlichungspflicht haben Mitglieder nach Art. III Abs. 3. GATS noch eine Berichtspflicht. Sie müssen den Rat für den Handel mit Dienstleistungen umgehend und mindestens einmal jährlich über die Einführung neuer oder die Änderung bestehender Gesetze, sonstiger Vorschriften oder Verwaltungsrichtlinien unterrichten, die den Handel mit Dienstleistungen wesentlich betreffen.

Nach diesem Prinzip ist VR China verpflichtet, nur die veröffentlichten und den anderen WTO-Mitgliedern verfügbaren Gesetze, Bestimmungen und andere Maßnahmen in Bezug auf Handel mit Gütern, Dienstleistungen, geistigem Eigentum oder Kontrolle der Devisen durchzuführen. ${ }^{264}$

\section{Zuständigkeit für den Technologietransfer in China}

Das Ministerium für Außenhandel und Wirtschaftliche Zusammenarbeit ist auf der Grundlage der Genehmigung durch den NVK auf dessen Sitzung im März 2003 abgelöst worden durch das neu gegründete Handelsministerium. Das neue MOC vereint MOFTEC, die Staatliche Kommission für Wirtschaft und Handel und die neu benannte Staatliche Kommission für Entwicklung und Reform. ${ }^{265}$

\section{Rechtsquellen}

Der Rechtsrahmen beim Technologietransfer besteht aus verschiedenen Rechtsquellen. Betroffen sind allgemeine Gesetze, die Verwaltungsrechtsnormen des Staatsrats, dem die Ministerien und Kommissionen unterstehen, lokale Verordnungen und Vorschriften, Rechtsnormen der Sonderwirtschaftszonen, Recht des Sonderverwaltungsgebietes und internationale Abkommen sowie sachbezogene Rechtsprechungen. Solche Gesetze, Bestimmungen und Regelungen sind aufgeteilt in zwei Bereichen bezüglich von ausländischen Direkt-Investitionen, Technologieeinfuhr und -Ausfuhr, Wettbewerbsrecht sowie dem Rechtschutz des geistigen Eigentums näher zu erläutern.

${ }^{263}$ Vgl. Stoll, 2006 , S. 224 f.

264 Protocol on the Accession of the People's Republic of China Part I 2 (C) 1

http://docsonline.wto.org/DDFDocuments/t/WT/L/432.doc, WT/ L/ 432

${ }^{265}$ Pattloch, S. 695; Huck, S. 7 


\section{Regelungen über Technologietransfer im zivilrechtlichen Bereich}

1. Die Allgemeinen Grundsätze des Zivilrechts

Die chin. AGZR 266 vom 12. April 1986 sind heutzutage das grundlegende Zivilgesetz in China, das eine Reihe für das gesamte Zivilrecht geltende Grundsätze und Verhaltensnormen enthält. ${ }^{267}$ Diese chin. AGZR sind weder das Zivilgesetzbuch noch nicht der Allgemeine Teil eines solchen. Sie bestehen aus 156 Paragraphen in neun Kapiteln, die folgende Überschriften haben: Grundprinzipien, Bürger (natürliche Personen), juristische Personen, Zivilrechtshandlung und Vertretung, zivile Rechte, zivile Haftung, Klageverjährung, Rechtsanwendung bei Zivilbeziehungen mit Auslandsberührung, ergänzende Regeln. ${ }^{268}$ Es ist zu erwähnen, dass z. B. die chin. AGZR die Rechte des geistigen Eigentums als Privatrechte direkt im fünften Kapitel, 3. Abschnitt verankert haben. Außerdem bilden allgemeine Vorschriften über die vertragsrechtlichen Pflichten der Vertragsparteien im sechsten Kapitel der chin. AGZR eine Rechtsgrundlage für das chinesische Vertragsgesetz und damit haben die chin. AGZR bei der Rechtsanwendung einen Rechtsvorrang vor dem Vertragsgesetz.

2. Technologietransfer nach dem Vertragsgesetz der VR China von 1999269

a. Einführung

Das geltende Recht zum Technologietransfer wurde schon vor der Konkretisierung eines Beitritts zur WTO als reformbedürftig und erweitungsfähig angesehen. ${ }^{270}$ Bislang musste grundsätzlich zwischen rein innerstaatlichem Technologietransfer und grenzüberschreitendem Technologietransfer unterscheiden werden. ${ }^{271}$ Dies widersprach dem Nichtdiskriminierungsprinzip der WTO. Deshalb ist die Gleichbehandlung durch die einheitlichen Bestimmungen im neuen chin. VG verkörpert worden.

Bis zur Verabschiedung des neuen Vertragsgesetzes, in Kraft seit dem 1. Oktober 1999, waren noch das Wirtschaftsvertragsgesetz zu beachten, in Kraft seit dem 1. Juli $1982^{272}$, und das Technologievertragsgesetz ${ }^{273}$, in Kraft seit dem 1.

\footnotetext{
${ }^{266}$ Allgemeine Grundsätze des Zivilrechts der VR China, verabschiedet auf der 4. Sitzung des 6. Nationalen Volkskongresses am 12. 4. 1986, die ergänzt werden durch „,versuchsweise durchgeführte Ansichten des Obersten Volksgerichts zu einigen Fragen der Anwendung der Allgemeinen Grundsätze des Zivilrechts der VR China“, am 26. 1.1988 von der Urteilskommission des Gerichts verabschiedet und am 2. 4. 1988 mit Fa (ban) fa 1988/6 den Gerichten mitgeteilt, Quelle: http://lehrstuhl.jura.uni-goettingen.de/chinarecht/zivilrecht.htm; Siehe dazu, Guo, Shoukang, Guoji jishu zhuanrang, 12 ff.; Münzel, Frank, Unternehmens- und Gesellschaftsrecht der VR China, S. $7 \mathrm{ff}$.

${ }^{267}$ Siehe dazu Liang, Huixing, Das chinesische Zivilrecht: Sein gegenwärtiger Zustand und der Erlass eines Zivilgesetzbuches, Heuser Robert(Hrsg.), Beiträge zum chinesischen Zivil- und Wirtschaftsrecht, S. 13 ff.; Shan, Xiaogang, S. 34 f.; Peng, Wanlin, S. 11; Wang, Hanbin, FZRB vom 6. 2. 1997.

${ }^{268}$ Liang, Huixing, Das chinesische Zivilrecht: Sein gegenwärtiger Zustand und der Erlass eines Zivilgesetzbuches, Heuser Robert(Hrsg.), Beiträge zum chinesischen Zivil- und Wirtschaftsrecht, S. 14. 269 Verabschiedet am 15.3.1999 von der 2. Sitzung des 9. Nationalen Volkskongresses; Gesetz: Renmin ribao 22.3.1999; Frank Münzel (Hrsg.) http://lehrstuhl.jura.uni-goettingen.de/chinarecht/vertrag.htm; Shan, GRURInt. 2000, S. $506 \mathrm{ff}$.

270 Zhang, Qiang(Hrsg.), Guoji Jishu Zhuanrang Jiufen yu Yufang Anli Fenxi, S. 20.

271 Vgl. Pattloch, GRURInt. 2003 Heft 8-9. S. 695.

272 Wirtschaftsvertragsgesetz vom 13. 12. 1982, Einführung von Liang, Huixing, Heuser, Robert(Hrsg.), Das
} 
November 1987, einschließlich seiner Durchführungsbestimmungen, in Kraft seit 15. März 1989, sowie das Außenwirtschaftsvertragsgesetz ${ }^{274}$ vom 21. März 1985, in Kraft seit dem 1. Juli 1985. Die drei Vertragsgesetze sind dann durch das einheitliche chin. VG abgelöst worden. Die strikte Zweiteilung des Rechts in die Inländer betreffende Regelungen und für Ausländer gültiges Recht ist in den letzten Jahren kontinuierlich durch die Verabschiedung neuer Gesetze zurückgedrängt worden. ${ }^{275}$

b. Anwendungsbereich des chin. VG

Eine der wesentlichen Grundlagen für zum Verständnis eines chinesischen Technologietransfervertrags auf den drei vorerwähnten Feldern des Handels, der Investition oder der technischen Unterstützung ist das moderne chinesische Vertragsgesetzbuch von 1999, das im 18. Kapitel in vier Abschnitte gegliederte Bestimmungen zum Abschluss von 4 unterschiedlichen Technikverträgen enthältt ${ }^{276}$, nämlich, Verträge über die Entwicklung von Techniken im 2. Abschnitt, Verträge über die Übertragung von Techniken im 3. Abschnitt sowie Verträge über technische Beratung und Verträge über technische Dienstleistungen im 4. Abschnitt.

Das Vertragsgesetz ist subsidiär gegenüber bestehenden Gesetzen, die vertragliche Regelungen enthalten, soweit diese in spezielleren Gesetzen gesonderte Berücksichtigung gefunden haben. ${ }^{277}$ Soweit gesetzlich nichts anderes bestimmt ist, können die Parteien eines Vertrags mit Auslandsbezug das bei der Regelung von Vertragsstreitigkeiten anzuwendende Recht frei wählen. ${ }^{278}$ Falls die Parteien keine Wahl getroffen haben, wird das Recht des Staates mit der engsten Verbindung zum Vertrag angewandt, das bei Technologietransferverträgen im Regelfall die VR China sein dürfte. ${ }^{279}$ Grundsätzlich gilt folgendes: Auf dem Gebiet der VR China zu erfüllende Verträge über chinesisch-ausländische mit gemeinsamem Kapital betriebene Unternehmen, über chinesisch-ausländische kooperativ betriebene Unternehmen und über chinesisch-ausländische gemeinsame Erschließung und Ausbeutung natürlicher Ressourcen wird das Recht der VR China angewandt. ${ }^{280}$

\section{c. Begriffserklärung}

Als Vertrag bezeichnet dieses Gesetz eine Vereinbarung, mit der zwischen Bürgern, juristischen Personen und anderen Organisationen als gleichberechtigten Subjekten eine Beziehung mit zivilrechtlichen Rechten und Pflichten errichtet,

Außenwirtschaftsrecht der Volksrepublik China, S. $49 \mathrm{ff}$.

273 Siehe dazu zum Technologievertragsgesetz, Shan, Xiaoguang, S. 36 ff.

274 Außenwirtschaftsvertragsgesetz vom 21. 3. 1985, Heuser, Robert(Hrsg.), Das Außenwirtschaftsrecht der Volksrepublik China, S. $72 \mathrm{ff}$.

${ }^{275}$ Pattloch, GRURInt. 2003 Heft 8-9. S. 696.

${ }^{276}$ Huck, Winfried, Rahmenbedingungen für den Technologietransfer von Deutschland nach China, S. 7.

$277 \S 123$ VG

278 § 126 VG

$279 \S 126 \mathrm{VG}$

${ }^{280} \S 126 \mathrm{VG}$ 
geändert oder beendet wird. ${ }^{281}$

Technikverträge nach chinesischem Recht sind Verträge, in denen für die Entwicklung und für die Übertragung von Techniken, für technische Beratung und für technische Dienstleistungen die gegenseitigen Rechte und Pflichten festgesetzt werden. ${ }^{282}$ Der Zweck für die Schließung der Verträge soll allerdings für den wissenschaftlich-technischen Fortschritt von Nutzen sein und die Umwandlung, Anwendung und Verbreitung wissenschaftlich-technischer Ergebnisse beschleunigen. ${ }^{283}$

d. Inhalt der Technikverträge

Der Inhalt von Technikverträgen wird den Parteien vereinbart. In der Regel enthalten Technikverträge folgende Punkte:284

i. Bezeichnung des Vorhabens;

ii. Inhalt, Bereich und Anforderungen des Vertragsgegenstandes;

iii. Plan, Ablauf, Fristen, Orte, Gebiete und Art und Weise der Ausführung;

iv. Geheimhaltung der technischen Informationen und Unterlagen;

v. Gefahrtragung;

vi. wem die technischen Ergebnisse gehören sollen, wie ihre Nutzungen verteilt werden;

vii. Abnahmenormen und Abnahmeverfahren;

viii. Preis bzw. Entgelt oder Gebrauchsgebühren und Zahlungsweise;

ix. Berechnung von Vertragsstrafen oder Schadenersatz;

x. Art und Weise der Beilegung von Streitigkeiten;

xi. Erklärung von Bezeichnungen und Fachausdrücken.

Nach den Vereinbarungen der Parteien können folgende Unterlagen als Bestandteile des Vertrages einbezogen, wie z.B. zur Vertragserfüllung in Bezug stehende Unterlagen zum technischen Hintergrund, die Beweisführung der Durchführbarkeit und Berichte zur technischen Bewertung, Aufgabenstellungen und Planurkunden für das Vorhaben, technische Normen und Regeln, erste Projektierungen und Schriftstücke zur Technologie und andere technische Schriftstücke und Akten. ${ }^{285}$ Wenn Technikverträge Patente betreffen, müssen die Bezeichnung der Erfindungsschöpfung, den Patentantragssteller und den Patentinhaber, Datum und Nummer des Antrags, die Nummer des Patents und die Gültigkeitsdauer des Patents vermerkt werden. ${ }^{286}$

e. Nichtigkeitsgrund

Der Vertrag, der Techniken rechtswidrig monopolisiert, den technischen Fortschritt behindert oder die technischen Ergebnisse anderer verletzt, ist nichtig. 287

\footnotetext{
$281 \S 2 \mathrm{VG}$

$282 \S 322 \mathrm{VG}$

$283 \S 323 \mathrm{VG}$

$284 \S 324 \mathrm{VG}$

285 § 324 Satz 2. VG

${ }^{286} \S 324$ Satz 3. VG

$287 \S 329 \mathrm{VG}$
} 
f. Verträge über die Entwicklung von Techniken

Gegenstand eines Entwicklungsvertrags ist Forschung und Entwicklung in Bezug auf neue Techniken, neue Produkte, neue Technologien und neue Materialien und deren Systeme. ${ }^{288}$ Zum Vertrag über die Entwicklung von Techniken gehören der Entwicklungsauftragsvertrag und der Entwicklungskooperationsvertrag. 289

g. Verträge über technische Beratung und Verträge über technische Dienstleistungen

$\mathrm{Zu}$ den Verträgen über technische Beratung gehören Verträge, nach denen für bestimmte technische Projekte die Beweisführung der Durchführbarkeit, technische Prognosen, technische Untersuchungen besonderer Fragen, analysierende und bewertende Berichte, etc. gestellt werden. ${ }^{290}$ Verträge über technische Dienstleistungen sind Verträge, nach denen eine Partei mit technischem Wissen für die andere Partei bestimmte technische Fragen löst; nicht dazu gehören Bauleistungsverträge und Werkverträge. ${ }^{291}$

h. Verträge über den Technologietransfer

Zum Gegenstand eines Vertrags zur Übertragung von Technologie gehört die Übertragung von Patenten, die Übertragung des Anmeldungsrechts von Patenten, die Übertragung des Know-hows und die Lizenz zur Anwendung von Patenten.292

In Bezug auf die Lizenzverträge hat China als Mitgliedstaat der WTO die Bestimmungen des Art. 40 TRIPS in dem chin. VG umzusetzen. Lizenzverträge, die den Wettbewerb beschränken, nachteilige Auswirkungen auf den Handel haben können und die Weitergabe und Verbreitung von Technologie behindern können, können danach als nichtig angesehen werden.

Nach $₫ 344$ chin. VG ist der Lizenzvertrag des Patentrechts nur während des Bestehens des Patentrechts wirksam. Nach dem Ablauf des Patentrechts oder wenn das Patentrecht als ungültig erklärt wird, darf der Patentrechtinhaber keine Lizenz mehr erteilen. Aber es ist nicht klar, ob der Gesetzgeber damit die Praktiken über die Verhinderung von Angriffen auf die Gültigkeit kontrollieren will.

$\mathrm{Zu}$ den Rücklizenzierungsverpflichtungen stellt $\ 354$ chin. VG ganz klar, dass die Vertragsparteien vereinbaren können, die abtrennbaren Verbesserungen an der lizenzierten Technologie nach dem Reziprozitätprinzip zu teilen. Ohne solche Vereinbarungen oder wenn die Vereinbarungen nach $\$ 61$ chin. VG immer noch unklar sind, haben die Lizenznehmer allein die Schutzrechte an den Verbesserungen.

Die Vorschriften über den Technologietransfervertrag im chin. VG brauchen noch mehr Aufmerksamkeit auf die wettbewerbswidrigen Praktiken in der

\footnotetext{
${ }^{288}$ Huck, Winfried, Rahmenbedingungen für den Technologietransfer von Deutschland nach China, S. 8.

$289 \S 330$ Abs. 2 VG

$290 § 356$ Abs. 1 VG

291 § 356 Abs. 2 VG

292 Siehe dazu § 342 Abs. 1 VG
} 
vertraglichen Lizenzierung zu werfen. Rechte und Pflichten der Vertragsparteien in den Technologietransferverträgen werden im folgenden vierten Teil behandelt.

3. Technologietransfer bei der Gründung der Unternehmen mit ausländischer Kapitalbeteiligung

Die in China gegründeten Gemeinschaftsunternehmen mit chinesisch-ausländischer Kapitalbeteiligung (Equity Joint Ventures), chinesisch-ausländische Kooperationsunternehmen (Contractual Joint Ventures; Vertragliche Joint Ventures) und Unternehmen rein ausländischen Kapitals (Foreign-capital Enterprises) werden in China zusammen als Unternehmen mit ausländischer Kapitalbeteiligung bezeichnet. ${ }^{293}$

Nach $\int 5$ des „,Gesetz der VR China über Gemeinschaftsunternehmen mit chinesisch-ausländischer Kapitalbeteiligung“ vom 1. Juli 1979, geändert am 4. April 1990 und 15. März $2001^{294}$ und S 8 des „Gesetz der VR China über chinesisch-ausländische Kooperationsunternehmen“vom 13. April 1988, geändert am 31. Oktober $2000^{295}$ können die gewerblichen Schutzrechte und Know-how als Kapitalbeteiligung in die Joint Ventures eingebracht werden.

In der Durchführungsverordnung zum GUG vom 22. Juli 2001296 werden die Kapitalbeteiligungsformen im Kapitel 4. und der Technologieimport im Kapitel 6. genauer erläutert. Nach \25 der Durchführungsverordnung zum GUG müssen die gewerblichen Schutzrechte oder Know-how, die die ausländischen Investoren als Kapitalbeteiligung in die Gemeinschaftsunternehmen einbringen, einer der zwei Voraussetzungen entsprechen. Entweder können sie wesentlich Funktionen oder Qualität der vorhandenen Produkte verbessern und damit die Produktionseffektivität erhöhen oder Rohstoff, Brennstoff oder Energie sparen.

Der Technologieimport bedeutet, dass das Gemeinschaftsunternehmen die gebrauchte Technologie von Dritten oder Gemeinschaftsunternehmer durch den Technologietransfer erhält. Die Verträge über den Technologietransfer bedürfen der Genehmigung der zuständigen Behörde ( $(43$ Durchführungsverordnung zum GUG). Ähnliche Regelungen finden sich auch in Durchführungsbestimmungen zum KUG vom 4. September $1995^{297}$ und Durchführungsbestimmungen vom 12. April $2001^{298}$ zum Gesetz der VR China über Unternehmen ausländischen Kapitals vom 12. April 1986, geändert am 31. Oktober $2000^{299}$.

\section{Wirtschaftsverwaltungsrechtliche Technologietransfer}

Regelungen zum

1. Das Außenhandelsgesetz der VR China von 2004

\footnotetext{
293 Vgl. Shan, Xiaoguang, S. 45.

294 Quelle: Englische Version,http://www.fdi.gov.cn/ltlaw/lawinfodisp.jsp?id=ABC00000000000005098\&appId=1

295 Quelle: Englische Version,http://www.fdi.gov.cn/ltlaw/lawinfodisp.jsp?id=ABC00000000000004158\&appId=1

${ }^{296}$ Quelle: Englische Version,http://www.fdi.gov.cn/ltlaw/lawinfodisp.jsp?id=ABC00000000000010431\&appId=1

297 Quelle: Englische Version,http://www.fdi.gov.cn/ltlaw/lawinfodisp.jsp?id=ABC00000000000003972\&appId=1

${ }^{298}$ Quelle: Englische Version,http://www.fdi.gov.cn/ltlaw/lawinfodisp.jsp?id=ABC00000000000008745\&appId=1

299 Quelle: Englische Version,http://www.fdi.gov.cn/ltlaw/lawinfodisp.jsp?id=ABC00000000000005097\&appId=1
} 
a. Einführung

Das am 6.4.2004 erlassene Außenhandelsgesetz ${ }^{300}$ hat seinen Ursprung in 1994.

Das die gesamten 90er Jahre bis 2001 de jure bestehende Außenhandelssystem wurde durch die „Vorläufigen Bestimmungen für das Kommissionssystem im Außenhandel“301, durch das „Außenhandelsgesetz der VR China“302 sowie eine Vielzahl weiterer Verwaltungsbestimmungen geregelt. ${ }^{303}$ Die Änderung bezweckt hauptsächlich, die chinesischen Außenhandelsvorschriften mit den WTO-Anforderungen zu harmonisieren. ${ }^{304}$

Das Außenhandelsgesetz ist von seiner Konzeption ein Rahmengesetz, das durch weitere, noch zu formulierende Bestimmungen klarere Konturen erhalten wird. ${ }^{305}$ In $\int 2$ AHG wird der Anwendungsbereich vorgeschrieben, dass es Anwendung auf den Außenhandel und den Schutz der handelsbezogenen Aspekte der Rechte des geistigen Eigentums findet. Im Sinne des Gesetzes bezieht sich der Außenhandel auf die Ein- und Ausfuhr der Güter und Technologie und den internationalen Handel mit Dienstleistungen.

b. Außenhandelsbetreiber

Im Beitrittsprotokoll zum WTO-Beitritt der VR China ist vereinbart, dass China sowohl bezüglich der Berechtigung selbst als auch dem Umfang nach Handelsberechtigungen erweitern soll. ${ }^{306}$ China hat diese Verpflichtungen durch neu geändertes AHG umgesetzt. Wesentliche Veränderung ist nach \8 AHG 2004, dass nunmehr auch natürliche Personen in China berechtigt sind, Außenhandel zu betreiben. Binnen drei Jahren nach dem Beitritt sollen alle Unternehmen in China das Recht erhalten, Güter in dem Zollgebiet der VR China zu handeln, mit der Ausnahme einiger speziell genannter Güter. ${ }^{307}$ Alle ausländischen Unternehmen und Einzelpersonen - auch diejenigen, die nicht in China registriert und investiert sind - sollen bezüglich des Rechts, Handel zu treiben, wie chinesische Unternehmen behandelt werden. ${ }^{308}$

Sämtliche Außenhandelsbetreiber müssen sich bei der für den Außenhandel zuständigen Abteilung des Staatsrats bzw. bei von ihr beauftragten Organen zur Registrierung anmelden. 309 Voraussetzung für die Geschäftstätigkeit im Außenhandel ist lediglich eine formale Registrierung. Nach $\int S$ 21, 22 der Verwaltungsmethode für die Handelsaktivitäten ausländisch investierter

\footnotetext{
300 Übersetzung und knapper Kommentar zum neuen Außenhandelsrecht von Heuser, Robert, Die Integration der WTO in das chinesische Außenhandelsrecht, CHINA aktuell 2004, S. 528 ff sowie von Müller, Mathias, Außenhandelsgesetz der VR China, ZChinR 2004, S. 250 ff.

${ }^{301}$ Die vorläufigen Bestimmungen für das Kommissionssystem im Außenhandel vom 29. August 1991, chinesisch-Englisch in: China Law and Practice, Vol. 5 (1991), Nr. 10, S. 48 ff.

${ }^{302}$ GWY GB 1994, S. 423 ff.; Deutsche Übersetzung in: Heuser, Robert (Hrsg.), Wirtschaftsreform und Gesetzgebung in der VR China, S. $376 \mathrm{ff}$.

303 Julius/Müller, ZChinR 2004, S. 215.

${ }^{304}$ Ganea, GRURInt. 2004, S. 889.

${ }^{305}$ Huck, S. 13.

306 Julius/Müller, ZChinR 2004, S. 216.

${ }^{307}$ Teil 1 Punkt 5 Ziffer 1 des Beitrittsprotokolls der VR China zur WTO; abgedruckt unter http://www.wto.org/english/thewto_e/acc_e/completeacc_e.htm\#chn

${ }_{308}$ Teil 1 Punkt 5 Ziffer 1, 2 des Beitrittsprotokolls der VR China zur WTO; abgedruckt unter http://www.wto.org/english/thewto_e/acc_e/completeacc_e.htm\#chn

$309 \S 9$ AHG
} 
Unternehmen vom 1. Juni $2004^{310}$ wird die Gründung ausschließlich ausländisch investierter Handelsunternehmen ab dem 11.12.2004 gestattet, deren Sitz nicht in bestimmten Wirtschaftszonen vorgeschrieben ist.

c. Der Im- und Export von Waren und Technologie

In $\int 14 \mathrm{AHG}$ ist der Grundsatz des freien Warenhandels vorgeschrieben, nach dem der Staat die freie Ein- und Ausfuhr von Gütern und Technologie gestattet, es sei denn, \11 AHG bestimmt, dass der Staat für einen Teil von Waren eine staatliche Verwaltung durchführen kann. Das bedeutet, dass nur bestimmte staatlich ermächtigte Unternehmen diese Waren im- oder exportieren dürfen. Außerdem bestehen noch die vom Grundsatz der Handelsfreiheit abweichenden Regelungen nach $\iint 15$ bis 17 AHG, die von den $\iint 18$ bis 23 AHG konkretisiert werden. ${ }^{311}$

$\int 15$ AHG räumt dem Staat die Möglichkeit ein, für einen Teil von frei importierbaren Waren ein Verfahren der automatischen Genehmigung einzuführen. ${ }^{312}$ \16 AHG stellt einen Katalog der Tatbestände dar, die ein Verbot oder eine Beschränkung des freien Waren- und Technologieverkehrs zulassen. Der ist eng an den Gründen für allgemeine Ausnahmen gem. Art. 20 des GATT 1994 orientiert. \ 17 AHG enthält eine weitere Ausnahme von der Warenverkehrsfreiheit, die dem Staat alle Maßnahmen zum Schutz der nationalen Sicherheit in Verbindung mit Materialien zur Kernspaltung und ihrer Derivate, Waffen, Munition und anderen Militärgütern, sowie in Kriegs- und Krisenzeiten gestattet. ${ }^{313}$ Diese Ausnahmen stehen im Einklang mit Art. 21 GATT 1994.

d. Vorschriften zum Schutz des geistigen Eigentums

Das neue Außenhandelsgesetz enthält im Kapitel 5 Vorschriften zum Schutz des geistigen Eigentums im Zusammenhang mit dem Außenhandel

$\int 29$ AHG verweist auf die einschlägigen Gesetze zum Schutz des geistigen Eigentums und räumt dem Handelsministerium MOC bestimmte Verwaltungskompetenzen ein, \30 AHG betrifft Regelungen bezüglich des Inhalts von Verträgen über die Lizenzierung der Schutzrechte des geistigen Eigentums und $\int 31$ AHG betrifft Maßnahmen Chinas gegenüber anderen Staaten in Zusammenhang mit dem Schutz geistigen Eigentums. ${ }^{314}$

2. Die Verwaltungsbestimmungen für den Technologieimport und -Export

a. Einführung

Nach Erlass des „Außenwirtschaftsvertragsgesetz der VR China“315 hat der Staatsrat am 24. Mai 1985 die „Bestimmungen über Verwaltung von

310 Englische Übersetzung in: China Law and Practice, Vol. 18, Nr. 4, S. 24 ff.

311 Julius/Müller, ZChinR 2004, S. 218.

312 Julius/Müller, ZChinR 2004, S. 219.

313 Julius/Müller, ZChinR 2004, S. 221.

314 Vgl. Julius/Müller, ZChinR 2004, S. 223.

315 Außer Kraft getreten durch das neue chin. VG; GWY GB 1985 Nr. 9 S. 217 ff. 
Technikimportverträgen“316 verkündet, die den im Außenwirtschafts- Verkehr wichtigsten Technologieimportvertragstypus einer Sonderregelung unterwarf. ${ }^{317}$ Am 20. Januar 1988 hat dasselbe Ministerium die Ausführungsregeln zur TIVB verkündet. ${ }^{318}$

Vor dem Hintergrund des WTO-Beitritts sind die TIVB und deren Ausführungsregeln durch die Verwaltungsbestimmungen für den Technologieimport und -Export ${ }^{319}$ ersetzt worden. Seit dem 1. Januar 2002 gilt die TIEVB vom 18. Dezember 2001, \55 chin. TIEVB. Daneben wurden durch den Staatsrat die Verwaltungsbestimmungen für verbotene oder beschränkte Importtechnologie, die Verwaltungsbestimmungen für verbotene oder beschränkte Exporttechnologie 320 sowie die Eintragungsregeln für Technologieimport- und Technologieexportverträge erlassen. ${ }^{321}$

b. Anwendungsbereich

Der Anwendungsbereich ist sehr weit gefasst: Er umfasst alle Arten von Übertragungsakten einschließlich Handel, Investitionen oder wirtschaftliche bzw. technische Kooperationen, und meint insbesondere die Übertragung von Patentrechten oder Patentanmelderechten, die Lizenzierung von Patentrechten, die Übertragung geheimen Know-hows, technische Dienstleistungen und jeden anderen Transfer von Technologie, $\$ 2$ chin. TIEVB ${ }^{322}$

c. Grundsatz des freien Verkehres der Technologie

Nach $\int 5$ chin. TIEVB sind die Einfuhr und Ausfuhr von Technik grundsätzlich frei und bedürfen keiner gesonderten öffentlich-rechtlichen Genehmigung, es sei denn, dass spezielle Bestimmungen ein besonderes Verfahren vorsehen, was nicht selten der Fall ist. Aber da sind freie, beschränkte und verbotene Techniken zu unterscheiden.

Nach $\iint 8,31$ chin. TIEVB ist die den Fällen in $\int S$ 16, 17 AHG entsprechenden Technologie von Einfuhr und Ausfuhr beschränkt oder verboten. Die zuständigen Behörden geben einen Katalog der von Einfuhr beschränkten oder verbotenen Techniken heraus. ${ }^{323}$ Nach $\$ \$ 9,32$ chin. TIEVB darf verbotene Technologie nicht importiert oder exportiert werden. Nach $\$ \int 10,33$ chin. TIEVB bedarf die Einfuhr oder Ausfuhr der beschränkten Technologie einer vorherigen Genehmigung durch die zuständigen Behörden.

\section{d. FIE}

In Sachen der Übertragung von Technologie als Sacheinlage in FIEs sind $\$ 22$ chin.

\footnotetext{
${ }^{316}$ GWY GB 1985 Nr. 16 S. 520 ff.; deutsche Übersetzung in: Dietz, S. 345 ff.; Heuser/Zhao, RIW 1985, S. 684 ff. 
TIEVB und entsprechende Vorschriften im GUG und KUG sowie Durchführungsverordnung zum GUG und Durchführungsbestimmungen zum KUG in Verbindung gesetzt. Technologietransfer kann durch die Einbringung einer entsprechenden Einlage bei der Gründung einer chinesischen Gesellschaft erfolgen. Nach \ 22 chin. TIEVB kann bei der Gründung einer FIE von ausländischen Investoren Technologie als Kapitalbeteiligung investiert werden.

e. Genehmigungsverfahren für die Lizenzerteilung

i. Freie Technologie

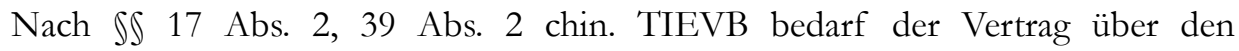
Technologietransfer bei freier Technik nur noch einer deklaratorischen Registrierung. Der Importeur freier Technologie muss zur Durchführung des Registrierungsverfahrens zum einen ein Onlineverfahren unter der Adresse http://info.ec.com.cn durchlaufen. ${ }^{324}$ Nach Vornahme der Onlineregistrierung muss der Importeur ein Anmeldeformular für einen Technologieimport zusammen mit einer Kopie des Vertrages und Nachweisen zur rechtlichen Stellung der Parteien bei der zuständigen Verwaltungsbehörde nach \18 chin. TIEVB einreichen. Nach $\int 19$ chin. TIEVB muss die zuständige Verwaltungsbehörde die Registrierung innerhalb von drei Werktagen vornehmen.

\section{ii. Beschränkte Technologie}

Im Unterschied zur freien Technologie kann ein Übertragungsvertrag in der Kategorie beschränkte Technologie erst mit Erteilung einer Genehmigung wirksam und für die Parteien bindend werden, SS 16, 38 chin. TIEVB. ${ }^{325}$

Der Importeur muss nach $\iint 11,12$ chin. TIEVB einen Antrag auf Erteilung eines sog. Letter of Intent stellen, dessen technische und handelsbezogene Aspekte die Verwaltungsbehörden überprüfen und innerhalb von 30 Tagen entscheiden müssen. Das MOC entscheidet innerhalb von 10 Werktagen nach Erhalt des unterzeichneten Vertrages und anderer für den jeweiligen Vertrag relevanter Dokumente nach $\int 14$ Abs. 1 chin. TIEVB über die Echtheit des Vertrages und die Genehmigung, $\int 14$ Abs. 2 chin. TIEVB. Der Vertrag wird erst wirksam mit Erteilung der Genehmigung nach $\int 16$ chin. TIEVB.

iii. Sonstiges

Das jeweilige Verwaltungsverfahren muss bei jeder Änderung der wesentlichen Inhalte eines registrierten oder genehmigten Transfers erneut durchlaufen werden, wobei bei einer Beendigung eines Technologievertrags eine bloße Benachrichtigung der Verwaltungsbehörde zur Eintragung genügt, \S 21, 43 chin.

\footnotetext{
${ }^{324}$ Vgl. §§ 3, 4 der von MOFTEC am 30. 12. 2001 verkündeten und am 1. 1. 2002 in Kraft getretenen Maßnahmen für die Eintragung für Technologieimport- und Technologieexportverträge. Gemäß § 7 der Maßnahmen enthält die Registrierung folgende Angaben: Nummer und Bezeichnung der Vertrages, die Namen des Übertragenden, Übertragungsempfängers sowie des Nutzers der Technologie, eine Übersicht über den Vertrag, die Vertragssumme, Art und Weise der Zahlungen, Devisenabrechnung und Kredite.

${ }^{325}$ Pattloch, GRURInt. 2003, S. 700.
} 
TIEVB. ${ }^{326}$ Gegen jede Art von Entscheidung seitens der Verwaltungsbehörden innerhalb dieses Verfahrens gibt $\int 53$ chin. TIEVB den beteiligten Parteien das Recht, Widerspruch bei der nächst höheren Behörde einzulegen oder direkt vor den Volksgerichten zu klagen.

\section{f. Haftung, Geheimhaltung}

Nach \ 24 chin. TIEVB haftet der Technologiegeber dafür, dass er der rechtmäßige Inhaber der gelieferten Technologie und hinreichend legitimiert ist, die Technologie zu übertragen oder zu lizenzieren. Außerdem hat der Technologiegeber zu garantieren, dass die von ihm gelieferte Technologie vollständig, fehlerfrei, wirksam und zur Erreichung der technischen Zielsetzung geeignet ist. ${ }^{327}$ Die beiden Vertragsparteien sind nach $\ 26$ chin. TIEVB im Rahmen ihrer vertraglichen Vereinbarungen zur Geheimhaltung verpflichtet.

g. Verbot wettbewerbswidriger Bestimmungen

Einer materiellen, inhaltlichen Prüfung werden die Technologieimportverträge vor allem im Hinblick auf das Verbot wettbewerbswidriger Bestimmungen unterzogen. Bislang waren wettbewerbswidrige Bestimmungen in $₫ 9$ TIVB geregelt. Nach $₫ 29$ chin. TIEVB verdienen folgende Punkte besondere Beachtung ${ }^{328}$ :

i. Kopplungsgeschäfte;

ii. Weitere Entrichtung von Nutzungsgebühren nach Ablauf des Patents;

iii. Beschränkung, die Technologie zu verbessern oder eine verbesserte Technologie zu nutzen;

iv. Beschränkung aus anderen Quellen Technologie zu erwerben, die der gelieferten ähnlich ist oder im Konkurrenz steht;

v. Ungerechte Beschränkung der Bezugskanäle und Quellen;

vi. Ungerechte Beschränkung der hergestellten Menge, Produktarten und Preisen;

vii. Ungerechte Beschränkung der Exportwege.

3. Vorschriften zur Lenkung ausländischer Investitionen am 11. Februar 2002

Der Zweck der Lenkungsrichtlinien ist darauf gerichtet, die Investitionen mit der volkswirtschaftlichen und sozialen Entwicklungsplanung in Einklang zu bringen. Die Vorschriften werden angewandt auf ausländische Investitionsprojekte, die in der Form der Foreign Invested Enterprises verfolgt werden sowie auf Projekte der Investition ausländischer Firmen in anderen Formen. Ausländische Investitionsprojekte werden in vier Kategorien, nämlich geförderte, gestattete, eingeschränkte und verbotene Projekte unterteilt.

Der Branchenkatalog zur Lenkung ausländischer Investitionen der VR China erfasst unterschiedliche Infrastrukturprojekte und Industrieanlagen. Der Katalog zur Lenkung ausländischer Investitionen in den vorherrschenden Industrien der Zentral- und Westregionen von 2000 unterscheidet nicht zwischen Kategorien,

${ }^{326}$ Pattloch, GRURInt. 2003, S. 699.

327 Vgl. Huck, S. 12.

${ }^{328}$ Vgl. Huck, S. 12.; Pattloch, GRURInt. 2003, S. 701 f. 
sondern zwischen Provinzen, in denen insgesamt 225 Industriebereiche gefördert werden.

\section{Dritter Teil: Der Rechtsschutz des geistigen Eigentums in der VR China}

Beim internationalen Technologietransfer spielt der Rechtsschutz des geistigen Eigentums eine Schlüsselrolle. In diesem Teil wird der Schwerpunkt auf das nationale Recht zum Schutz des geistigen Eigentums gelegt. Als Mitglieder der WTO und WIPO hat VR China die Verpflichtungen, sein nationales Recht in Einklang mit den internationalen Rechtsschutzstandards zu bringen. ${ }^{329}$ Insbesondere beim Technologietransfer ist der gewerbliche Rechtsschutz von wichtiger Bedeutung. Im Vergleich zum gewerblichen Eigentum aus deutscher Sicht kann der Mangel am Schutz des geistigen Eigentums herausgefunden werden, um die Schutzrechtbestimmungen in China zu verbessern.

\section{A. Grundzüge über den Rechtsschutz des geistigen}

\section{Eigentums}

I. Grundzüge des internationalen Rechtsschutzes des geistigen Eigentums

1. Theorien zum Patentschutz 330

Nach Machlup ${ }^{331}$ lassen sich die verschiedenen Ansätze zur Befürwortung des Patentschutzes in vier Prinzipien untergliedern, die sich nicht voneinander abgrenzen, sondern sich überschneiden.

a. Naturrechtstheorie

Die Naturrechtstheorie geht davon aus, dass der Mensch ein unbedingtes Eigentumsrecht an seinen eigenen Ideen hat. Die Allgemeinheit sei moralisch verpflichtet, dieses Eigentumsrecht anzuerkennen und zu schützen. Eigentum bedeutet individuelle Herrschafts- und Nutzungsbefugnis. ${ }^{332}$ Nach Osterrieth ${ }^{333}$ knüpft die Eigentumstheorie an der aus der Naturrechtstheorie stammenden

\footnotetext{
${ }^{329}$ Siehe dazu zu den Verpflichtungen Chinas zum Schutz des geistigen Eigentums, Trade Policy Review, WT/TPR/S/161, S. $145 \mathrm{ff}$.

${ }^{330}$ Vgl. Chrocziel, S. 13 f.; Beier, GRURInt. 1970, S. 1 f.

331 Machlup, GRURAusl. 1961, S. 377

${ }^{332}$ Schmidt-Diemitz, GRURInt. 1988, S. 287.

333 Osterrieth, Patentrecht, S. 5. Fn. 6.
} 
Überzeugung an, dass dem Erfinder diese Befugnis gebührt, Dritte von der Nutzung auszuschließen.

b. Belohnungstheorie

Nach der Belohnungstheorie hat der Erfinder einen Anspruch auf eine Gegenleistung der Allgemeinheit je nach der Nützlichkeit seines geleisteten Dienstes als Belohnung. Die Belohnungstheorie geht insoweit weiter als die Eigentumstheorie als sie die Belohnung nicht allein in der Gewährung eigentumsähnlicher Rechte an der Erfindung sieht, sondern auch eine andere Art der Gegenleistung, etwa eine finanzielle Belohnung oder einen anderen geldwerten oder ideellen Vorteil anerkennt. ${ }^{334}$

c. Anspornungstheorie

Wie die Naturrechts- und Belohnungstheorie verleiht die Anspornungstheorie dem Erfinder den Patentschutz und damit verbundenen wirtschaftlichen Vorteil. Aber das Patentrecht wird nicht nur als Erfüllung des Anspruchs des individuellen Erfinders angesehen, sondern als der Anreiz für technische Erfindertätigkeit zugunsten der technischen Entwicklung im Interesse der Allgemeinheit gestellt. Nicht das Interesse des Erfinders, sondern das Interesse der Allgemeinheit steht im Vordergrund. ${ }^{335}$ Für den Gesetzgeber war der Gedanke, dass das Patent die Erfindertätigkeit anspornt und damit den technischen Fortschritt fördert, in fast allen Ländern der maßgebliche Gesichtspunkt für die Einführung des Patentschutzes. ${ }^{336}$

d. Vertragstheorie

Nach der Vertragstheorie wird ein Austauschvertrag zwischen dem Erfinder und der Allgemeinheit abgeschlossen, auf Grund dessen der Erfinder den Inhalt seiner Erfindung in der Anmeldung offenbart, um dafür den zeitlich befristeten Ausschließlichkeitsschutz für die gewerbliche Verwertung dieser Erfindung zu begründen. Deshalb wird die Vertragstheorie auch als Offenbarungstheorie genannt.

2. Grundprinzipien der gewerblichen Schutzrechte

a. Numerus clausus

Nach dem sachenrechtlichen Grundsatz des numerus clausus gibt es nur eine geschlossene Zahl von Arten dinglicher Rechte und übriger sachenrechtlicher Institute. ${ }^{337}$ Eine neue Entwicklung oder neue Schöpfung, die nicht unter die anerkannten Kategorien fällt, bleibt daher grundsätzlich schutzlos. ${ }^{338}$

\footnotetext{
${ }^{334}$ Osterrieth, Patentrecht, S. 5. Fn. 7.

335 Beier, GRURInt. 1970, S. 3.

336 Beier, GRURInt. 1970, S. 3.

337 Weinmann, S. 414.

${ }^{338}$ Chrocziel, S. 19.
} 


\section{b. Territorialitätsprinzip}

Die Schutzwirkungen der gewerblichen Schutzrechte sind nach dem Territorialitätsprinzip ${ }^{339}$ auf das Hoheitsgebiet des jeweiligen Staates begrenzt. ${ }^{340}$ Der Grundsatz der Territorialität des Patentrechts führt dazu, dass der Erfinder eine Vielzahl von Patentanmeldungen vornehmen muss, deren Schicksal sich nach den jeweils geltenden nationalen Bestimmungen richtet, wenn er von der Wirkung des Patentschutzes in allen intendierten Märkten profitieren will. ${ }^{341}$ Das Territorialitätsprinzip trägt dem politischen Charakter der Schutzrechtsanerkennung dadurch Rechnung, dass es als zwingende Kollisionsnorm den Erwerb, Umfang, Bestand und Verlust eines Schutzrechts dem Recht des Staates unterwirft, für dessen Gebiet Technologieschutz begehrt wird. ${ }^{342}$

c. Erschöpfungsprinzip (Konsumption)

Erschöpfung ${ }^{343}$ meint Verbrauch des Patentrechts hinsichtlich eines bestimmten patentgemäßen Erzeugnisses. ${ }^{344}$ Sie ist Rechtsfolge der Tatsache, dass der Patentinhaber oder ein mit dessen Zustimmung handelnder Dritter den betreffenden mit dem Patent unter Schutz gestellten körperlichen Gegenstand oder ein bestimmtes unmittelbares Erzeugnis eines patentierten Verfahrens an einem Ort innerhalb eines bestimmten Gebiets in den Verkehr gebracht hat. ${ }^{345}$ Aufgrund des Territorialitätsprinzips kann die Erschöpfung nur innerhalb des räumlichen Geltungsbereiches des Schutzrechtes eintreten. ${ }^{346}$

\section{d. Prioritätsprinzip}

Das Prioritätsprinzip ${ }^{347}$ besagt, dass derjenige, der in einem der Mitgliedstaaten der Pariser Verbandsübereinkunft die Anmeldung für ein Erfindungspatent, ein Gebrauchsmuster, ein gewerbliches Muster oder Modell sowie eine Marke vorschriftsmäßig hinterlegt hat für die Hinterlegung in den anderen Ländern während einer Frist von zwölf Monaten bei Erfindungspatenten und Gebrauchsmustern und einer Frist von sechs Monaten für die Anmeldung gewerblicher Muster oder Modelle bzw. Marken ein Priorität genießt. ${ }^{348}$ Das Prioritätsprinzip spielt im internationalen gewerblichen Rechtsschutzsystem eine wesentliche Rolle. Es soll verhindern, dass eine Erstanmeldung oder deren Benutzung gegenüber einer Nachanmeldung des gleichen Anmelders, die in einem

\footnotetext{
339 Siehe dazu, Chrocziel, S. 24 f.; Ullrich, GRURInt. 1995, S. 623 ff.; Böcksriegel, GRUR 1999, S. 1 ff.; Lundstedt, GRURInt. 2001, S. 103 ff.

${ }^{340}$ Vgl. Benkard, § 15, Rn. 224. S. 612.

341 Vgl. Osterrieth, Patentrecht, S. 27.

${ }^{342}$ Vgl. Ullrich, GRURInt. 95, S. 625.

343 Siehe dazu zum Erschöpfungsprinzip, Krasser, S. 818 ff.; Benkard, § 9 Rn. 15 ff. S. 371 ff.; Staehelin, S. 28 ff.; Beier, GRURInt. 1989, S. 603 ff.; Beier, GRURInt. 1996, S. 1 ff.; Gaster, GRURInt. 2000, S. 571 ff.; Löwenheim, GRUR 1982, S. 461 ff.; Löwenheim, GRURInt. 1996, S.307 ff.; Mager, GRUR 1999, S. 637 ff.; Sack, GRUR 1999, S. 193 ff.; Sack, GRURInt. 2000, S. 610 ff.; Schatz, GRURInt. 1970, S. 207 ff.; Ullrich, GRURInt. 1983, S. 370 ff.; Blasek, GRURInt. 2006, S. $568 \mathrm{ff}$.

344 Benkard, § 9, Rn. 16. S. 372.; BGH GRUR 97, S. 116 - Prospekthalter.

${ }_{345}$ Benkard, § 9, Rn. 16. S. 372.; BGH GRUR 2001, S. 223 - Bodenwaschanlage.

346 Vgl. Chrocziel, S. 21.; Benkard, § 9, Rn. 18 ff. S. 373 ff.

347 Siehe dazu zum Prioritätsprinzip, Chrocziel, S. 25 ff.; Brandi-Dohrn/Gruber/Muir, S. 17 ff.; Krasser, S. 512 ff.

348 Vgl. Art. 4 PVÜ; Benkard, Einleitung, Rn. 51. S. 21.
} 
anderen Staat für die gleiche Erfindung hinterlegt wurde, schädlicher Stand der Technik wird. ${ }^{349}$

3. Internationale Übereinkommen in Bezug auf das gewerbliche Eigentum ${ }^{350}$ Der gewerbliche Rechtsschutz unterliegt in großem Maße internationalem Einfluss. Gewerbliche Schutzrechte kann jeder Staat nach dem Territorialitätsprinzip nur für den räumlichen Bereich gewähren, auf den sich seine Souveränität erstreckt. ${ }^{351}$ Aber in der Regel beabsichtigen Inhaber von gewerblichen Schutzrechten, nicht nur begrenzt auf ein Land Schutz zu erlangen, sondern sie haben ein starkes Interesse daran, diese Schutzrechte in vergleichbarer, besser noch identischer Form, in mehreren Ländern geschützt zu erhalten. ${ }^{352}$ Deshalb bedarf es der internationalen Verständigung über den gewerblichen Rechtsschutz durch internationale Vereinbarungen. Die folgenden Vereinbarungen stellen nur die wichtigsten existierenden internationalen Regelungen dar.

a. Pariser Verbandsübereinkunft

i. Einführung

Da sich der rein bilaterale Schutz von Anfang an als unzulänglich erwies, schlossen sich im Jahre 188311 Staaten im Rahmen der Pariser Verbandsübereinkunft ${ }^{353}$ zu einem Verband zusammen, der den Schutz der Erfindungen, der Muster und Modelle, der Fabrik- oder Handelsmarken und der Handelsnamen sowie die Unterdrückung des unlauteren Wettbewerbs in den Verbandsstaaten zum Inhalte hatte. ${ }^{354}$ Die PVÜ stellt deshalb auf internationaler Ebene die grundlegende Verfassung des gewerblichen Eigentums dar. ${ }^{355}$ Sie hat allerdings kein einheitliches Recht auf diesen Gebieten geschaffen, lässt auch keine transnationalen Immaterialgüterrechte entstehen, sondern hat die nationalen Verschiedenheiten und das Territorialitätsprinzip weithin unangetastet gelassen. ${ }^{356}$

ii. Grundprinzipien der PVÜ

Ihr tragender Grundsatz ist die Inländerbehandlung (Assimilationsprinzip) ${ }^{357}$ : Jedes Verbandsland gewährt den Angehörigen aller anderen Verbandsländer, auch wenn sie in jenem Land weder Wohnsitz noch Niederlassung haben, in Bezug auf den gewerblichen Rechtsschutz dieselben Vorteile wie den eigenen Staatsangehörigen. ${ }^{358}$

Die Bestimmungen über die Unionspriorität 359 sind von besonderer

\footnotetext{
${ }^{349}$ Vgl. Brandi-Dohrn/Gruber/Muir, S. 17.

350 Siehe dazu, Chrocziel, S. 54 ff.; Thaler, S. 71 ff.; Holyoak/Torremans, S. 24 ff.; Schmidt-Diemitz, GRURInt. 1988, S. 292.; Fischer, S. 373 ff.; Hubmann/Götting, S. 39 ff.;

351 Vgl. Krasser, S. 78.

352 Vgl. Chrocziel, S. 54.

353 Siehe dazu zur Geschichte der PVÜ, Beier, GRURInt. 1983, S. 339 ff.

354 Art. 1 PVÜ; Vgl. Osterrieth, Patentrecht, S. 29.

355 Vgl. Chrocziel, S. 54.;

${ }^{356}$ Vgl. Hubmann/Götting, S. 98.

357 Art. 2 und 3 PVÜ

358 Vgl. Krasser, S. 79.; Hubmann/Götting, S. 98.; Chrocziel, S. 54.; Osterrieth, Patentrecht, S. 30.

359 Art. 4 PVÜ
} 
Bedeutung: Die erste Anmeldung einer Erfindung in einem Staat der PVÜ begründet ein Prioritätsrecht. ${ }^{360}$

Die PVÜ enthält darüber hinaus schutzrechtliche Mindestrechte und begründet damit für die Verbandsländer eine Rechtsvereinheitlichung. ${ }^{361}$ Solche besonderen Rechte sind z. B. das Recht auf Erfindernennung ${ }^{362}$, das Verbot des Ausübungszwangs ${ }^{363}$, die Einführung einer Mindestfrist von drei Jahren nach Patenterteilung für die Erteilung von Zwangslizenzen wegen unterlassener oder ungenügender Ausübung ${ }^{364}$ sowie der telle-quelle-Schutz der Marke ${ }^{365}$.

b. Patentzusammenarbeitsvertrag

Für Patente und Gebrauchsmuster ist neben der PVÜ der Patentzusammenarbeitsvertrag zu beachten. Der PCT wurde am 19. 6. 1970 in Washington als multilateraler Vertrag von ursprünglich 20 Mitgliedstaaten unterzeichnet.

Auf seiner Grundlage ist es seit 1. Juni 1978 möglich, durch Einreichung einer einzigen internationalen Anmeldung in einem Vertragsstaat ein Patent oder Gebrauchsmuster in allen von ihm bestimmten Mitgliedstaaten zu erwerben. ${ }^{366}$ Gegenstand der Vereinheitlichung ist lediglich das Anmeldeverfahren für internationale Patentanmeldungen und die Neuheitsrecherche. ${ }^{367}$

\section{c. Haager Musterabkommen}

Auf dem Gebiet des Geschmackmusterschutzes ist neben der PVÜ das HMA zu berücksichtigen. Es gilt nur für einen engeren Kreis der Verbandsstaaten der PVÜ und ergänzt deren Bestimmungen. ${ }^{368}$ Die am 2. 2. 1999 verabschiedete, aber noch nicht in Kraft getretene Genfer Fassung des HMA unterzeichneten bislang 29 Staaten. ${ }^{369}$

Durch eine internationale Registrierung von Mustern oder Modellen kann der Hinterleger den Musterschutz in allen Vertragsstaaten des Haager Musterabkommens erwerben. ${ }^{370}$ Das HMA schafft ebenso wenig wie die PVÜ ein einheitliches Musterrecht, sondern es wird lediglich die besondere Hinterlegung in jedem einzelnen Verbandsstaat erspart. ${ }^{371}$ Der Schutz, der durch diese internationale Hinterlegung zu erreichen ist, beträgt maximal 15 Jahre. ${ }^{372}$

d. Madrider Markenabkommen

Auf dem Gebiet des Markenschutzes wird die PVÜ für einen engeren Kreis von

${ }^{360}$ Vgl. Fischer, S. 375.;

361 Vgl. Osterrieth, Patentrecht, S. 32.

362 Art. 4 ter PVÜ

363 Art. 5 A Abs. 1 PVÜ

364 Art. 5 A Abs. 4 PVÜ

365 Art. 6 quinquies PVÜ

${ }^{366}$ Vgl. Hubmann/Götting, S. 99.; Krasser, S. 81.; Osterrieth, Patentrecht, S. 33.

367 Osterrieth, Patentrecht, S. 33.

${ }^{368}$ Hubmann/Götting, S. 101.

369 Hubmann/Götting, S. 101.; Siehe dazu zur Neufassung, Mansani, GRURInt. 2000, S. 896 ff.

${ }^{370}$ Fischer, S. 379.

${ }^{371}$ Vgl. Hubmann/Götting, S. 101.; Fischer, S. 379.

${ }^{372}$ Vgl. Chrocziel, S. 56. 
Verbandsstaaten durch das MMA und das Protokoll zum MMA ergänzt. ${ }^{373}$

Aufgrund des MMA kann der Inhaber eines Warenzeichens oder einer Dienstleistungsmarke auf Antrag durch eine internationale Registrierung bei der WIPO in Genf Schutz in denjenigen Ländern erhalten, die dem Abkommen beigetreten sind. ${ }^{374}$ Der Schutz wird für 20 Jahre eingeräumt.

Das Protokoll zum MMA vom 27. Juni 1989 dient dazu, die Einbeziehung der Länder in das MMA zu ermöglichen, die grundsätzlich dem MMA nicht beitreten würden - es findet keine Anwendung, wenn ein Land bereits Mitglied des MMA ist. ${ }^{375}$

\section{e. TRIPS-Übereinkommen}

Die Bedeutung der gewerblichen Schutzrechte im Rahmen der Neuordnung der internationalen Wirtschaftsbeziehungen findet ihren Ausdruck in dem TRIPS 376 vom 15. April 1994, welches am 1. 1. 1995 in Kraft getreten ist. ${ }^{377}$ Es stellt ein besonderes völkerrechtliches Abkommen im Zusammenhang mit der Gründung der WTO dar.

Dem TRIPS geht es um die Schaffung gemeinsamer Standards der handelsbezogenen Aspekte der Immaterialgüterrechte. ${ }^{378}$ Nach Art. 2 TRIPS sind die Mitgliedstaaten verpflichtet, die Art. 1-12 sowie Art. 19 der PVÜ in der Fassung von 1967 zu beachten. ${ }^{379}$ Neben dem Grundsatz der Inländerbehandlung 380 , Angehörige der anderen Mitglieder beim Schutz geistigen Eigentums nicht weniger günstig zu behandeln als ihre eigenen Angehörigen, bestehen die Verpflichtung der Mitgliedsstaaten zur Weitergabe von Vergünstigungen (Meistbegünstigungsgrundsatz) 381 und das Gebot, die niedergelegten Mindeststandards zum Schutz des gewerblichen Eigentumsschutzes einzuhalten ${ }^{382} .383$

Erwähnenswert ist der Erschöpfungsgrundsatz in Art. 6 TRIPS. Gemäß dem Grundsatz der internationalen Erschöpfung kann der Rechtsinhaber die Einfuhr der Ware, welche er oder ein Lizenznehmer in einem Drittland produziert hat, nicht mehr untersagen. ${ }^{384}$ Nach allgemein herrschender Meinung wird Art. 6 TRIPS dahin interpretiert, dass dieses Übereinkommen auf eine Regelung der internationalen Erschöpfung verzichtet und es jedem Mitgliedstaat überlassen habe, sich für das ihm günstigere System zu entscheiden. 385

\footnotetext{
${ }^{373}$ Vgl. Hubmann/Götting, S. 102.

${ }^{374}$ Vgl. Fischer, S. 376.

375 Vgl. Chrocziel, S. 55.

376 Siehe dazu zum TRIPS-Abkommen, Staehelin, S. 14 ff.; Verma, IIC 1996, S. 331 ff.; Dreier, GRURInt. 1996, S. 205 ff.; Faupel, GRURInt. 1990, S. 255 ff.

377 Vgl. Osterrieth, Patentrecht, S. 34.

${ }^{378}$ Benkard, Internationaler Teil, Rn. 77. S. 48.

379 Vgl. Kasser, S. 85 f.; Osterrieth, Patentrecht, S. 35.

380 Art. 3 TRIPS

381 Art. 4 TRIPS

382 Art. 1 Abs. 1 Satz 2 TRIPS

383 Vgl. Benkard, Internationaler Teil, Rn. 77. S. 48.; Krasser, S. 86.

${ }^{384}$ Staehelin, S. 28.

385 Vgl. Straus, GRURInt. 1996, S. 193.; Pacón, GRURInt. 1995, S. 878.; Siehe dazu, zum Grundsatz der internationalen Erschöpfung und Parallelimport, Yu, GRURInt. 2000, S. 619 ff.; zum Problem der Erschöpfung im TRIPS, Ullrich, GRURInt. 1995, S. 634 ff.; Soltysinski, GRURInt. 1996, S. 318 ff.
} 


\section{Bedeutung des Patentschutzes für den technischen Fortschritt}

Im Rahmen der Rechtsmittel zur Förderung der technischen Entwicklung kommt dem gewerblichen Rechtsschutz im allgemeinen und dabei insbesondere dem Schutz von Erfindungen durch Patente oder ähnliche Rechtstitel, aber auch dem Schutz von Warenzeichen und dem Schutz gegen unlauteren Wettbewerb, besondere Bedeutung zu.

\section{Anreiz für Forschung und Entwicklung}

Die maßgebliche Grundlage dieser Wirkungen ist die Ausschließlichkeit des Patentrechts. ${ }^{386}$ Ohne die Aussicht auf Patentschutz und das damit verbundene Nachahmungsverbot und Verwertungsmonopol wären viele Unternehmen nicht bereit, das Investitionsrisiko für neue technische Entwicklungen zu übernehmen. ${ }^{387}$

Einerseits weckt das Patentsystem die Hoffnung auf Patentschutz und materielle Belohnung der erfinderischen Tätigkeit, andererseits zwingt den Erfinder, rasch zu handeln und seine Erfindung gegenüber dem Patentamt in einem frühen Stadium offen zu legen, um Wettbewerbern gegenüber die Priorität zu erlangen. ${ }^{388}$

2. Förderung der Anwendung und Verbreitung technischen Wissens

Der Informationseffekt, der dem Patentsystem innewohnt, mit anderen Worten die frühzeitige Offenlegung der Erfindung zugunsten der Öffentlichkeit, die durch das Patentgesetz erzwungen wird, macht neue Technologie wesentlich rascher allgemein bekannt. ${ }^{389}$ Neue Anregungen für Forschungsarbeiten gehen von der zwangsläufigen Offenlegung der Patentschriften aus und zugleich steigert sich der Informationsfluss. 390 Das Bestehen von Patentschutz erhöht sich auch die Bereitschaft des Inhabers neuer Technologie, deren Benutzung mit anderen durch den Abschluss von Lizenzverträgen zu teilen.

3. Patentschutz als Instrument des internationalen Technologietransfers

Die Erfindung kann risikolos nicht nur im eigenen Betrieb verwertet, sondern an andere übertragen und lizenziert werden, der in ihr verkörperte technische Fortschritt breitet sich aus, das Patent wird zu einem der wichtigsten Instrumente des Technologietransfers. ${ }^{391}$ Ein effektiver Rechtsschutz im Falle der Patentverletzung stellt ein wichtiges Kriterium für Investoren dar. Die meisten Entwicklungsländer stehen heute keineswegs mehr so feindlich dem Patentsystem gegenüber, wie dies noch in den 80er Jahren der Fall war. Durch eine Stärkung der Rechte des Patentinhabers haben einige Entwicklungsländer eine erhebliche Verbesserung des Investitionsklimas und damit eine Verbesserung der Intensität

\footnotetext{
${ }^{386}$ Beier, GRURInt. 1979, S. 234.

387 Beier, GRURInt. 1979, S. 234.

388 Vgl. Pfanner, GRURInt.1983, S. 364.

389 Pfanner, GRURInt. 1983, S. 364.

390 Vgl. Oppenländer, GRUR 1977, S. 363.

391 Vgl. Beier, GRURInt. 1979, S. 234.
} 
des Technologietransfers erreicht. ${ }^{392}$

\section{Rechtsschutz des geistigen Eigentums und Kartellrecht}

1. Das Spannungsverhältnis zwischen Patent und Kartellrecht

Auf den ersten Blick besteht ein gewisser Widerspruch zwischen dem Rechtsschutz des geistigen Eigentums einerseits und dem andererseits durch das Kartellrecht verfolgten Ziel, freien Wettbewerb zu garantieren. Während gewerbliche Schutzrechte ihrem Inhaber für einen gewissen zeitlichen Rahmen ein alleiniges Recht zur Nutzung und damit ein gesetzliches Verwertungsmonopol gewähren, dient das Kartellrecht dem Erhalt der Wettbewerbsfreiheit, der Verhinderung von Monopolen und damit der unverminderten wirtschaftlichen Betätigung des Einzelnen. ${ }^{393}$

Dieser Widerspruch geschah vor dem Hintergrund des Konzepts des vollkommenen Wettbewerbs. Heute ist man vom Konzept des vollkommenen Wettbewerbs abgerückt. Stattdessen wird die Wettbewerbspolitik durch das Konzept des dynamischen, unvollkommenen Wettbewerbs geleitet. ${ }^{394}$

Der dynamische, unvollkommene Wettbewerb zeichnet sich durch die Möglichkeit des Bestehens partieller Monopolsituationen aus, die jedoch bedingt durch ständige Innovationen der Wettbewerber alsbald ihren wirtschaftlichen Wert verlieren. ${ }^{395}$ Die Innovationen werden durch Patente und andere technische Schutzrechte gefördert, indem diese dem Inhaber die Möglichkeit geben, unter dem Schutze des Patents die Beträge zu erwirtschaften, die er benötigt, um in Zukunft weiter innovativ tätig sein zu können. ${ }^{396}$

2. Der Missbrauchstatbestand

Die neoliberale Schule sieht die Innovations- und Diffusionshemmenden Wirkungen des Patentschutzes als so gravierend an, dass sie für die Abschaffung des Patentsystems oder wenigstens für die erhebliche Herabsetzung des Patentschutzes durch Einführung eines allgemeinen Lizenzzwangs plädiert. ${ }^{397}$ Die Gefahr Wettbewerbsbeschränkender Missbräuche des geltenden Patentsystems wird vor allem in drei Tatbeständen gesehen:

a. Schutzeffekt als Innovationshemmnis

Hier handelt es sich um Patentmonopole und Patenthäufungen, nämlich, die hohe Konzentration des Patentbesitzes in einigen Industriezweigen und bei einigen Unternehmen. Vorratspatente, die anwendungsreife Erfindungen darstellen und vor allem aus marktstrategischen Überlegungen noch nicht wirtschaftlich genutzt

\footnotetext{
392 Vgl. Osterrieth, Patentrecht, S. 8.

393 Vgl. Ulmer-Eilfort/Schmoll, S. 6.

394 Vgl. Pfaff/Osterrieth, Rn. 141 ff. S. 33.

395 Osterrieth, Patentrecht, S. 11.

396 Osterrieth, Patentrecht, S. 11.

${ }^{397}$ Vgl. Oppenländer, Patentschutz und Wettbewerb im Innovationsprozess, in: Oppenländer, S. 62.; Siehe dazu auch zu den hemmenden Wirkungen des Patentwesens, Oppenländer, GRUR 1977, S. 366 ff.; Pfanner, GRURInt. 1983, S. 364 ff.; Beier, Patentschutz - weltweit Grundlage technischen Fortschritts und industrieller Entwicklung, in: Oppenländer, S. 29 ff.
} 
werden, haben somit für den technischen Fortschritt eine verzögernde Wirkung. ${ }^{398}$

b. Schutzeffekt als Diffusionshemmnis

Die Monopolstellung, die durch Patenthäufungen erzielt wird, kann durch die reaktive Patentpolitik gefestigt werden. Hier handelt es sich darum, das Entstehen fremder Schutzrechte zu verhindern oder bereits bestehende Schutzrechte Dritter zu vernichten oder mindestens einzuschränken. ${ }^{399}$ Sperrpatente sollen in der Regel verhindern, dass Konkurrenten auf einem bestimmten Markt existieren können.

c. Lizenzpolitik der Unternehmen

Die Verweigerung der Lizenzerteilung kann einen Missbrauch einer Marktherrschenden Stellung darstellen. ${ }^{400}$ Der Patentinhaber kann auch aufgrund seiner Patentmonopole Wettbewerbsbeeinträchtigende Regelungen für die Übernahme von Verpflichtungen durch den Lizenznehmer in den Lizenzverträgen festlegen.

\section{B. Rechtsschutz des geistigen Eigentums in China im Vergleich mit dem Immaterialgüterrecht in Deutschland}

\section{Schutz des Patentrechts ${ }^{401}$}

\section{Einführung}

Das erste chinesische Patentgesetz ${ }^{402}$ trat am 1. April 1985 in Kraft. ${ }^{403}$ Mit der Zustimmung des Staatsrates hatte das Staatsamt für geistiges Eigentum am 19. Januar 1985 die Durchführungsbestimmungen zum Patentgesetz verabschiedet. ${ }^{404}$ Arbeiten an einer Neuregelung des alten PatG 1984 hatten bereits im Jahre 1988 begonnen. ${ }^{405}$ Am 4. September 1992 ist der Beschluss über die Änderung des chinesischen Patentgesetzes verabschiedet worden. ${ }^{406}$ Das erstmalig revidierte Patentgesetz ${ }^{407}$ mit seinen neuen Durchführungsbestimmungen ${ }^{408}$ ist am 1 .

\footnotetext{
${ }^{398}$ Vgl. Oppenländer, Patentschutz und Wettbewerb im Innovationsprozess, in: Oppenländer, S. 66.

399 Vgl. Oppenländer, Patentschutz und Wettbewerb im Innovationsprozess, in: Oppenländer, S. 69.

${ }^{400}$ Vgl. Osterrieth, Patentrecht, S. 13 f.

401 Siehe dazu zur Entwicklung des chinesischen Patentrechts, Guo, GRURInt. 1997, S. 949 ff.

402 Siehe dazu zum ersten chinesischen Patentgesetz, Guo, GRURInt. 1985, S. 1 ff.; ders. IIC 1985, S. 367 ff.;

Steinmann, M., Grundzüge des chinesischen Patentrechts

${ }^{403}$ Am 12. März 1984 wurde es vom Ständigen Ausschuß des VI. NVK auf seiner 4. Sitzung erlassen; Vgl. GWY GB 1984 Nr. 6 S. 164 ff.; Deutsch in Dietz, S. 196 ff.

404 GWY GB 1985 Nr. 3 S. 53 ff.; Deutsch in Dietz, S. 211 ff.

405 Vgl. Tetz, RIW 1993, S. 474.; Zhuanlifa xiuzhengan liyu jingji fazhan he duiwai jiaoliu, Renmin Ribao, 7. 9.

1992 - Die Neufassung des Patentgesetzes wirkt sich vorteilhaft auf die Entwicklung der Wirtschaft und den Außenhandel aus; Revision of Patent Law, Schweiz-China II/1992, S. 26.

${ }^{406}$ Verabschiedet vom Ständigen Ausschuß des VII. NVK auf seiner 27. Sitzung, Vgl. GWY GB 1992 Nr. 24 S. $934 \mathrm{ff}$.

407 GWY GB 1992 Nr. 24 S. 938 ff.; Deutsch in Tetz, RIW 1993, S. 477 ff.

408 GWY GB 1992 Nr. 30 S. 1305 ff.
} 
Januar 1993 in Kraft getreten. ${ }^{409}$

Am 25. August 2000 wurde eine zweite PatG-Änderung 410 verabschiedet. Aus Anlass der zweiten PatG-Änderung von 2000 hat der Staatsrat am 15. Juni 2001 neue Durchführungsbestimmungen ${ }^{411}$ erlassen, die am 1. Juli 2001 in Kraft getreten sind und die bisherigen Bestimmungen ersetzen. Die Überarbeitung des chin. PatG steht in einer Linie mit weiteren Anstrengungen Chinas, sich bei der Gesetzgebung im Bereich des gewerblichen Rechtsschutzes und Urheberrechts dem internationalen Standard anzunähern. ${ }^{412}$

Durch diese zwei Änderungen hat das chin. PatG wesentliche Verbesserungen erlebt und Mängel beseitigt, die die inländische Patentpraxis erschwert haben. So wurde z. B. in dem Bestreben, das Verfahren vor dem Patentamt so reibungslos wie möglich zu gestalten, der Komplex „Patentanfechtung“, also die Bestimmungen betreffend Einspruch bzw. Widerruf und Nichtigkeitsverfahren, gleich zweimal komplett umgestaltet. ${ }^{413}$

Das chin. PatG sieht seit seinem Bestehen drei Arten von Patenten vor, nämlich Patente für „Erfindungen“ ${ }^{414}$ (Faming), die eine Sachprüfung durchlaufen, sowie Patente für „Gebrauchsmuster“415 (Shiyong Xinxing) und "Geschmacksmuster“416 (Waiguan Sheji), die nur formal geprüft werden. ${ }^{417}$ Nach Art. 45 chin. PatG (jetzt Art. 42) wurde bereits 1992 die Laufzeit von Erfindungspatenten von 15 auf 20 Jahre und von Gebrauchs- und Geschmacksmusterpatenten von 5 auf 10 Jahre angehoben. 418

Die materiellrechtlichen Anforderungen in Bezug auf Patentfähigkeit entsprechen im Wesentlichen den deutschen Regelungen. ${ }^{419}$ Erforderlich sind Neuheit ${ }^{420}$, erfinderische Tätigkeit und gewerbliche Anwendbarkeit. ${ }^{421}$ Nach Art. 25 chin. PatG sind wissenschaftliche Entdeckungen, Regeln und Verfahren für gedankliche Tätigkeiten, Diagnose- und Therapieverfahren, Tierarten und Pflanzensorten sowie Stoffe, die durch Kernumwandlung gewonnen sind, vom Patentschutz ausgenommen.

Im deutschen Recht sind Patent, Gebrauchsmuster und Geschmacksmuster getrennt in drei unterschiedlichen Gesetzen geregelt. Nach $\$ 1$ GeschmMG steht

\footnotetext{
409 Siehe dazu zum PatG 1992, Guo, GRURInt. 1997, S. 949 ff.; Shan, Xiaoguang, S. 35.ff; Tetz, RIW 1993, S. 474 ff.; Yin, IIC 1993, S. 189 ff.; Scheil, GRURInt. 1996, S. 999 ff.; Yu, GRURInt. 2000, S. 620.; Ganea, GRURInt. 2002, S. 686 ff.; Thaler, S. $148 \mathrm{ff}$.

410 Aktuelle Fassung abgedruckt in GWY GB 2000 Nr. 30 S. 9 ff.; Deutsche Übersetzung in GRURInt. 2001, S. $541 \mathrm{ff}$.

411 Neue Fassung abgedruckt in GWY GB 2001 Nr. 23 S. 7 ff.; Deutsche Übersetzung in GRURInt. 2002, S. 243 ff.

412 Vgl. Tetz, RIW 1993, S. 474.; Shan, Xiaoguang, S. 35.; Zheng, Chengsi, S. 235.

413 Ganea, GRURInt. 2002, S. 687.

${ }^{414}$ Siehe dazu oben, Erster Teil B I.

415 Siehe dazu oben, Erster Teil B II.

416 Siehe dazu oben, Erster Teil B III.

417 Vgl. Ganea, GRURInt. 2002, S. 687. Scheil, GRURInt. 1996, S. 1000 f.

418 Vgl. Tetz, RIW 1993, S. 477.; Ganea, GRURInt. 2002, S. 702.

419 Scheil, GRURInt. 1996, S. 1000.

420 Neuheitsschädlich sind druckschriftliche Vorveröffentlichungen weltweit sowie nationale offenkundige Vorbenutzungen, Art. 22 Abs. 2 PatG; nicht neuheitsschädlich sind dagegen unveröffentlichte Voranmeldungen desselben Anmelders.

${ }^{421}$ Art. 22 chin. PatG
} 
dem Urheber ein Ausschließlichkeitsrecht zu. ${ }^{422}$ Der Geschmacksmusterschutz unterscheidet sich vom Patentschutz dadurch, dass er nicht technischen Handlungsanweisungen, sondern ästhetischen, d. h. auf den Formen- und Farbensinn wirkenden Gestaltungen gewerblicher Erzeugnisse gilt. ${ }^{423}$ Hier wird nicht näher behandelt.

Nach $₫ 9$ Satz 1 PatG $₫ 11$ Abs. 1 GebrMG ist allein der Patentinhaber oder Inhaber eines Gebrauchsmusters befugt, die patentierte Erfindung oder den Gegenstand des Gebrauchsmusters zu benutzen. Das Gebrauchsmuster ist sozusagen ein Teil des Patentsystems.

\section{Patentrechtsverletzungen}

a. im deutschen Recht

Eine Verletzung des Schutzrechts liegt in der Vornahme einer in $\iint 9$ bis 13 PatG, 11 bis 14 GebrMG verbotenen Benutzungshandlung. ${ }^{424}$

i. Die unmittelbaren Benutzungshandlungen

\ 9 Satz 2 PatG gibt abschließend die unmittelbaren Benutzungshandlungen an, die Dritte ohne Zustimmung des Patentinhabers nicht vornehmen dürfen, wenn nicht bestimmte Ausnahmebestände vorliegen. ${ }^{425}$ Die Ausnahmebestände sind in den $\iint 9$ a-c, 11 bis 13 PatG geregelt. $\int 9$ Satz 2 unterscheidet die allein dem Patentinhaber vorbehaltenen und Dritten verbotenen unmittelbaren Benutzungshandlungen nach dem verschiedenen Gegenstand des Patents: Erzeugnis (Nr. 1)oder Verfahren (Nr. 2 und 3). ${ }^{426}$

Zum Benutzungsrecht und den vorbehaltenen Handlungen (Verbietungsrecht) aus dem Gebrauchsmuster gelten die gleichen Grundsätze wie beim Patent. ${ }^{427}$ Nach $\int 11$ Abs. 1 Satz 2 GebrMG ist es jedem Dritten verboten, ohne Zustimmung des Gebrauchsmusterinhabers ein erfindungsgemäß beschafftes Erzeugnis herzustellen, anzubieten, in Verkehr zu bringen oder zu gebrauchen oder zu den genannten Zwecken einzuführen oder zu besitzen. ${ }^{428}$ Bestimmungen über den Verfahrensschutz fehlen, weil Gebrauchsmuster Verfahren nicht zum Gegenstand haben können. ${ }^{429}$

ii. Verwendung von Mitteln zur Erfindungsbenutzung (mittelbare Verletzung) Die in $\ 9$ PatG vorgesehenen Wirkungen des Patents werden durch die Bestimmungen des $\ 10$ PatG ergänzt. Nach $\ 10$ Abs. 1 PatG umfasst der Tatbestand der mittelbaren Patentverletzung objektive und subjektive

\footnotetext{
${ }^{422}$ Siehe dazu zum Geschmacksmuster, Nirk/Kurtze, S. 73 ff.

${ }^{423}$ Vgl. Krasser, S. 15.

${ }^{424}$ Hubmann/Götting, S. 218.

425 Vgl. Benkard, § 9 Rn. 27, S. 380.

${ }^{426}$ Benkard, § 9 Rn. 27, S. 380.

427 Busse, § 11 GebrMG Rn. 3, S. 1578.

${ }^{428}$ Vgl. Krasser, S. 768.

${ }^{429}$ Busse, § 11 GebrMG Rn. 3, S. 1578.
} 
Voraussetzungen. ${ }^{430}$ Handelt es sich um allgemein im Handel erhältliche Erzeugnisse, kommt mittelbare Patentverletzung nur in Betracht, wenn der Dritte den Belieferten bewusst zur Patentverletzung veranlasst ( $\$ 10$ Abs. 2 PatG).431 In Abs. 3 ist bestimmt, dass Personen, die die in $₫ 11$ Nr. 1 bis 3 PatG genannten Handlungen vornehmen, im Sinne des Abs. 1 nicht als benutzungsberechtigt gelten. Für Gebrauchsmuster enthält $\ 11$ Abs. 2 GebrMG eine dem $\ 10$ PatG entsprechende Regelung. ${ }^{432}$

iii. Erlaubte Benutzungshandlungen

a). Handlungen im privaten Bereich zu nichtgewerblichen Zwecken

Nach $\iint 11$ Nr. 1 PatG, 12 Nr. 1 GebrMG erstreckt sich die Wirkung des Patents und des Gebrauchmusters nicht auf Handlungen, die im privaten Bereich zu nicht-gewerblichen Zwecken vorgenommen werden.

b). Handlungen zu Versuchszwecken

Die Wirkung des Patents erstreckt sich nicht auf Handlungen zu Versuchszwecken, die sich auf den Gegenstand der patentierten Erfindung beziehen $(\mathbb{\Omega} 11 \mathrm{Nr} .2$ PatG). $\ 12$ Nr. 2 GebrMG gilt entsprechend.

c). Einzelzubereitung von Arzneimitteln

Nach $₫ 11$ Nr. 3 PatG sind die unmittelbare Einzelzubereitung von Arzneimitteln in Apotheken auf Grund ärztlicher Verordnung sowie Handlungen gestattet, welche die auf diese Weise zubereiteten Arzneimittel betreffen. ${ }^{433}$

d). Einrichtungen an ausländischen Fahrzeugen

Nicht patent- oder gebrauchsmusterverletzend sind nach Maßgabe des $₫ 11 \mathrm{Nr}$. 4 bis 6 PatG, auf die $\int 12 \mathrm{Nr}$. 3 GebrMG verweist, Handlungen in Bezug auf Einrichtungen an Schiffen, Luft- oder Landfahrzeugen, die vorübergehend oder zufällig ins Inland gelangen. 434

iv. Vorbenutzungsrecht

Eine Benutzung der Erfindung, die im Inland vor deren Anmeldung zum Patent begonnen oder hinreichend vorbereitet worden ist, darf nach Maßgabe des $\ 12$ PatG trotz Patenterteilung ohne Zustimmung des Patentinhabers fortgesetzt werden. ${ }^{435}$ Nach $\ 13$ Abs. 3 GebrMG ist $\ 12$ PatG im Gebrauchsmusterrecht entsprechend anwendbar.

v. Staatliche Benutzungsordnung

\footnotetext{
430 Vgl. Benkard, § 10 Rn. 3, S. 437.

431 Busse, § 10 PatG Rn. 22, S. 315.

${ }^{432}$ Krasser, S. 831.

${ }^{433}$ Vgl. Krasser, S. 816.

434 Krasser, S. 816.

435 Krasser, S. 846.; Siehe dazu zum Vorbenutzungsrecht, Benkard, § 12 Rn. 1 ff. S. 462 ff.; Busse, § 12 PatG Rn. 14 ff. S. 329 ff.
} 
Nach \13 Abs. 1 Satz 1 PatG kann die Bundesregierung anordnen, dass die Erfindung im Interesse der öffentlichen Wohlfahrt benutzt werden soll. Die Anordnung ist nach $\ 13$ Abs. 3 Satz 3 PatG dem im Register als Patentinhaber Eingetragenen vor Benutzung der Erfindung mitzuteilen. Ferner kann die zuständige oberste Bundesbehörde oder in ihrem Auftrag eine untergeordnete Behörde gem. \13 Abs. 1 Satz 2 PatG im Interesse der Sicherheit des Bundes die Benutzung einer patentierten Erfindung anordnen. Der Patentinhaber hat nach \ 13 Abs. 3 Satz 1 PatG gegen den Bund Anspruch auf angemessene Vergütung.

b. im chinesischen Recht

i. Anmaßung fremden und nicht bestehenden Patentrechts

Was genau unter dieser Anmaßung fremden Patentrechts zu verstehen ist, wurde in Art. 84 der Durchführungsbestimmungen in Form einer abschließenden Aufzählung einzelner Tatbestände definiert. ${ }^{436}$ Anmaßungstatbestände sind demnach das Anbringen der Patentnummer eines anderen auf den selbst hergestellten oder verkauften Erzeugnissen, die irreführende Verwendung der Patentnummer eines anderen in der Werbung und in Verträgen sowie die Fälschung oder Veränderung der Patentzertifikate, Patentunterlagen oder Anmeldeunterlagen eines anderen. ${ }^{437}$

Der Tatbestand, ein nicht patentiertes Erzeugnis oder Verfahren für ein patentiertes Erzeugnis oder Verfahren auszugeben, wurde 1992 in den früheren Art. 63 chin. PatG eingeführt und ist seit der Änderung von 2000 in einem eigenen Art. 59 chin. PatG geregelt. 438 Die Aufzählung einzelner Tatbestände der Anmaßung nicht bestehenden Patentrechts umfasst die Herstellung oder den Verkauf von mit einem Patenthinweis versehenen, jedoch nicht patentierten Erzeugnissen, das fortgesetzte Anbringen des Patenthinweises auf Erzeugnisse nach dem Erlöschen des Patentrechts, das Bezeichnen von nicht patentierter Technologie als patentierte Technologie in der Werbung oder in Verträgen und das Fälschen oder Verändern von Patentzertifikaten, Patentunterlagen oder Anmeldeunterlagen. ${ }^{439}$

\section{ii. Patentverletzungen}

Bereits Art. 11 Abs. 1 chin. PatG 1984 gab dem Patentinhaber eines Patents das ausschließliche Recht, die patentierten Produkte zu produzieren, vertreiben, benutzen oder importieren sowie beim Verfahrenspatent das patentierte Verfahren für Produktions- oder Geschäftszwecke anzuwenden. Liegt eine Patentverletzung vor, dann kann der Patentinhaber die Unterlassung der Patentverletzung und den dadurch entstandenen Schadensersatz nach Art. 57 Abs. 1 chin. PatG in Anspruch nehmen. Wenn das Erzeugnis, das von einem Dritten genutzt oder verkauft wird, nach dem patentierten Verfahren hergestellt wurde, dann kann der Patentinhaber

\footnotetext{
${ }^{436}$ Vgl. Ganea, GRURInt. 2002, S. 705.

437 Art. 84 der Durchführungsbestimmungen des chin. PatG von 2001

438 Ganea, GRURInt. 2002, S. 705.

439 Art. 85 der Durchführungsbestimmungen des chin. PatG von 2001
} 
die Verletzung seines Verfahrenspatents geltend machen. Nach Art. 57 Abs. 2 chin. PatG hat derjenige, der dasselbe Erzeugnis herstellt, das bei der Herstellung seines Erzeugnisses angewandte Verfahren nachzuweisen. Dies führt nach der Beweislastverteilung im chinesischen Zivilprozess zu einer Art Beweislastumkehr, denn nach $\ 64$ des chinesischen Zivilprozessgesetzes hat grundsätzlich jede Partei für ihr Vorbringen die Beweise zu liefern. ${ }^{440}$

iii. Gebrauchsmusterverletzungen

Nach Art. 57 Abs. 2 chin. PatG kann das Volksgericht oder die Patentverwaltungsbehörde den Patentinhaber im Zuge eines Gebrauchsmusterverletzungsstreits auffordern, bei der Patentverwaltungsbehörde des Staatsrates einen Recherchenbericht über das streitgegenständliche Gebrauchsmuster erstellen $\mathrm{zu}$ lassen. Um mehrfache Anträge auf Recherchenberichte zu ein und demselben Muster zu vermeiden, ist deshalb nur der Patentinhaber antragsberechtigt. ${ }^{441}$

iv. Ausnahmen

In Art. 63 chin. PatG werden Umstände aufgezählt, die nicht als Patentverletzung angesehen werden.

a). Art. 63 Abs. 1 Ziff. 1 chin. PatG

Nach Art. 63 Abs. 1 Ziff. 1 chin. PatG stellen Gebrauch, Verkauf und Verkaufsangebot von bereits verkauften und mit Zustimmung des Patentinhabers hergestellten oder importierten Patentprodukten und Verfahrenserzeugnissen keine Verletzungshandlungen dar. Die Neuregelung soll denjenigen Rechtssicherheit verschaffen, die rechtmäßig eingeführte Ware im Inland gebrauchen oder in Verkehr bringen. ${ }^{442}$ Jedoch enthält diese keine ausdrückliche Regelung zur Frage, ob Parallelimporte verboten sein sollen. ${ }^{443}$

b). Art. 63 Abs. 1 Ziff. 2 chin. PatG

Wenn derjenige, der vor dem Tag der Patentanmeldung bereits ein identisches Erzeugnis hergestellt, ein identisches Verfahren angewandt oder die notwendigen Vorbereitungen zur Herstellung oder Anwendung getroffen hat, die Herstellung oder Anwendung ausschließlich im ursprünglichen Umfang weiterführt. ${ }^{444}$

c). Art. 63 Abs. 1 Ziff. 3 chin. PatG

Wenn das betreffende Patent in einem ausländischen Transportmittel, das vorübergehend das Hoheitsgebiet, die Hoheitsgewässer oder den hoheitlichen Luftraum Chinas passiert, gemäß einem zwischen dem Land, dem das ausländische Transportmittel angehört, und China geschlossenen Abkommen oder

\footnotetext{
${ }^{440}$ Vgl. Steinmann, S. 208.

441 Vgl. PatG-Kommentar, S. 324 ff.

${ }^{442}$ PatG-Kommentar, S. $354 \mathrm{ff}$.

443 Siehe dazu zu den Parallelimporten, Yu, GRURInt. 2000, S. 619 ff.; Blasek, GRURInt. 2006, S. 568 ff.

444 GRURInt. 2001, S. 547.
} 
gemäß einem internationalen Vertrag, dem beide Länder angehören, oder gemäß dem Grundsatz der Gegenseitigkeit für die eigenen Bedürfnisse des Transportmittels in seinen Vorrichtungen und Einrichtungen benutzt wird. ${ }^{445}$

d). Art. 63 Abs. 1 Ziff. 4 chin. PatG

Das betreffende Patent wird ausschließlich für die Zwecke der wissenschaftlichen Forschung und für Versuchszwecke benutzt.

e). Art. 63 Abs. 2 chin. PatG

Wer ein patentiertes Erzeugnis oder ein unmittelbar aufgrund eines patentierten Verfahrens gewonnenes Erzeugnis für Produktions- oder gewerbliche Zwecke benutzt oder verkauft, ohne zu wissen, dass es ohne Zustimmung des Patentinhabers hergestellt und verkauft worden ist, haftet nicht für Schadensersatz, wenn er den rechtsmäßigen Bezug dieses Erzeugnisses nachweisen kann. ${ }^{446}$

3. Zivilrechtlicher Schutz

Der zivilrechtliche Schutz umfasst die Verteidigung gegen gegenwärtige und drohende zukünftige Rechtsverletzungen sowie die Beseitigung der Folgen von begangenen unerlaubten Handlungen. ${ }^{447}$

a. im deutschen Recht

i. Unterlassungsanspruch

Wer das Schutzrecht entgegen den $\$ \int 9-13$ PatG benutzt, kann nach $₫ 139$ Abs. 1 PatG vom Verletzten auf Unterlassung in Anspruch genommen werden. ${ }^{448}$ Wer den Vorschriften der $\$ \int 11$ bis 14 GebrMG zuwider ein Gebrauchsmuster benutzt, kann nach $\llbracket 24$ Abs. 1 GebrMG vom Verletzten auf Unterlassung in Anspruch genommen werden. ${ }^{449}$

ii. Schadensersatzanspruch

Bei schuldhaftem Verhalten kann der Schutzrechtsinhaber nach $₫ 139$ Abs. 2 PatG, 24 Abs. 2 GebrMG Schadensersatz verlangen. ${ }^{450}$

iii. Beseitigungsanspruch

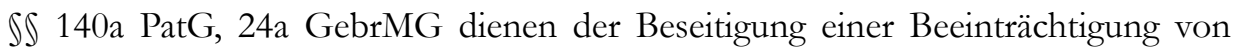
Rechten aus dem Patent oder Gebrauchsmuster. Der Verletzte kann in den Fällen der $\iint 139$ PatG, 24 GebrMG verlangen, dass im Besitz oder Eigentum des Verletzers befindliche Erzeugnis, das Gegenstand des Patents oder Gebrauchsmusters ist, vernichtet wird, es sei denn, dass der durch die Rechtsverletzung verursachte Zustand der Erzeugnisse auf andere Weise beseitigt

445 GRURInt. 2001, S. 547.

446 GRURInt. 2001, S. 547.

447 Troller, Immaterialgüterrecht II, S. 965.

448 Ensthaler, S. 133.

449 Krasser, S. 869.

450 Ensthaler, S. 133. 
werden kann und die Vernichtung für den Verletzer oder Eigentümer im Einzelfall unverhältnismäßig ist. ${ }^{451}$

iv. Auskunftsanspruch

Derjenige, der eine patentierte Erfindung oder ein Gebrauchsmuster widerrechtlich benutzt, kann vom Verletzten auf unverzügliche Auskunft über die Herkunft und den Vertriebsweg des benutzten Erzeugnisses in Anspruch genommen werden.

v. Umkehr der Beweislast

Für Verfahrenspatente über ein neues Erzeugnis stellt $\ 139$ Abs. 3 PatG eine Beweislastregel auf. Danach gilt bis zum Beweis des Gegenteils das gleiche von einem anderen hergestellte Erzeugnis als nach dem patentierten Verfahren hergestellt. ${ }^{452}$

b. im chinesischen Recht

i. Ermutigung der Parteien zur Absprache

In Art. 57 chin. PatG wird ausdrücklich bestimmt, dass sich die Parteien vor Einleitung eines Verletzungsprozesses darum bemühen sollten, in Absprache miteinander zu einer Lösung zu gelangen. ${ }^{453}$ So sollen eigentlich einsichtige Parteien zu einer für beide Seiten kostengünstigen Verhandlungslösung ohne Inanspruchnahme öffentlicher Ressourcen ermuntert werden. ${ }^{454}$ Jedoch ist die vorherige Absprache nicht zwingende Voraussetzung für die Einleitung eines Verfahrens, da eine Klage vor Gericht oder vor einer Patentverwaltungsbehörde nicht nur dann eingereicht werden kann, wenn die Absprache ergebnislos bleibt, sondern auch dann, wenn die Absprache nicht gewünscht wird. ${ }^{455}$

\section{ii. Haftung für Rechtsverletzung}

a). Unterlassung

Nach Art. 61 chin. PatG kann ein Patentinhaber oder ein sonst Interessierter, der den begründeten Nachweis erbringt, dass eine Verletzung seines Patentrechts, die ein Dritter gerade begeht oder im Begriff ist zu begehen, seinen rechtmäßigen Rechten und Interessen schwer zu behebenden Schaden zufügen kann, vor Erhebung einer Klage beim Volksgericht beantragen, Maßnahmen zur Anordnung der Unterlassung der Verletzungshandlung und zur Sicherung des Vermögens zu ergreifen. 456

Im Vergleich zum Unterlassungsanspruch im deutschen Recht wird die Anordnung der Unterlassung der Verletzungshandlung nach Art. 61 chin. PatG als

451 Vgl. Krasser, S. 871.

452 Vgl. Hubmann/Götting, S. 218.; Siehe dazu Benkard, § 139 Rn. 118 ff. S. 1458 ff.

453 Vgl. Ganea, GRURInt. 2002, S. 702.

454 PatG-Kommentar, S. 317.

455 Art. 57 chin. PatG; Ganea, GRURInt. 2002, S. 702.

${ }^{456}$ GRURInt. 2001, S. 546. 
die Institution der einstweiligen Verfügung betrachtet.

b). Schadensersatz

Es obliegt gem. Art. 57 chin. PatG nur noch den Volksgerichten, in einem Patentverletzungsstreit den Schadensersatz zu bestimmen.

c). Haftung aus chin. AGZR 457

Die Haftung für die Verletzung von Rechten des geistigen Eigentums wird in der Praxis auch aus den $\iint 118$ und 134 der chin. AGZR hergeleitet. 458

$\int 118$ chin. AGZR verpflichtet den Verletzer derartiger Rechte zur Einstellung der Verletzung, zur Beseitigung ihrer Auswirkungen und zum Schadensersatz.

$\int 134$ chin. AGZR regelt die zivilrechtliche Haftung in allgemeiner Form, wobei insgesamt zehn Formen der Haftung vorgesehen sind:

(1) die Einstellung von Verletzungen;

(2) die Beseitigung von Behinderungen;

(3) die Beseitigung von Gefahren;

(4) die Rückgabe von Vermögensgütern;

(5) die Wiederherstellung des ursprünglichen Zustandes;

(6) Reparatur, erneute Herstellung oder Austausch;

(7) Schadenersatz;

(8) die Zahlung von Vertragsstrafe;

(9) die Beseitigung von Auswirkungen und die Wiederherstellung des Rufes sowie (10) eine Entschuldigung.

4. Verwaltungsrechtlicher Schutz

a. Allgemeines

Es wird als eine chinesische Besonderheit angesehen, dass es dem Verletzten in Art. 57 chin. PatG zur Wahl gestellt wird, entweder vor einem Volksgericht oder vor einer Patentverwaltungsbehörde Klage einzureichen. ${ }^{459}$ In der VR China sind nämlich die Gerichte und bestimmte Behörden nebeneinander für die Durchsetzung von Rechten des geistigen Eigentums verantwortlich. ${ }^{460}$ Das international eher selten anzutreffende zweispurige System der Rechtsdurchsetzung durch Behörden und Gerichte hat sich in der Praxis offenbar bewährt, so dass auch die jüngsten Änderungen des Patentrechts nur eine schrittweise Stärkung der Justiz gegenüber den Behörden erkennen lassen. ${ }^{461}$

b. Patentverwaltungsbehörde

Art. 78 der Durchführungsbestimmungen hat ausführlich geregelt, dass

\footnotetext{
457 Siehe dazu oben, Zweiter Teil B II.

458 Siehe dazu zur Haftung für Rechtsverletzungen, Liu, GRURInt. 1999, S. 491 ff.

459 Vgl. Ganea, GRURInt. 2002, S. 702.

460 Vgl. Liu, GRURInt. 1999, S. $488 \mathrm{ff}$.

${ }^{461}$ Ganea, GRURInt. 2002, S. 702.
} 
Patentverwaltungsbehörden im Sinne des chin. PatG und dessen Durchsetzungsbestimmungen Patentverwaltungsbehörden sind, die von den Regierungen der Provinzen, Selbstverwaltender Autonomiegebiete, Selbstverwaltender Städte oder der Städte, die aus Bezirken bestehen und eine große Menge Arbeit an der Patentverwaltung haben und in der Lage sind, mit solcher Arbeit umzugehen.

c. Behördliche Schutzmaßnahmen

Nach Art. 57 chin. PatG können die Patentverwaltungsbehörden die Einstellung einer Patentrechtsverletzung anordnen. Innerhalb 15 Tagen nach Erhalt der Mitteilung kann der Betroffene nach dem chinesischen Verwaltungsverfahrensgesetz vor einem Volksgericht klagen. Die Behörden dürfen nur noch in der Frage des Schadensersatzes auf Antrag der Parteien schlichten. Darüber hinaus steht in den Art. 58 und 59 chin. PatG die Entscheidungsbefugnis den Behörden zu, im Falle der Anmaßung eines fremden oder eines nicht bestehenden Patentrechts öffentliche Richtigstellung, Herausgabe unrechtmäßig erlangten Einkommens und Geldstrafe anzuordnen. ${ }^{462}$

Außerdem ist in Art. 79 der Durchführungsbestimmungen geregelt, dass die Patentverwaltungsbehörden in Streitfragen, die die Zugehörigkeit des Patentrechts, die Erfinder- oder Entwicklereigenschaft, die Belohnung oder Vergütung für dienstliche Erfindungsschöpfungen sowie die unvergütete Nutzung der Erfindung im Veröffentlichungszeitraum betreffen, lediglich schlichten dürfen.

\section{Strafrechtlicher Schutz}

a. im deutschen Recht

Nach \ 142 PatG ist die vorsätzliche unmittelbare Verletzung eines für die Bundesrepublik Deutschland geltenden Patents oder ergänzenden Schutzzertifikats strafbar. ${ }^{463}$ Die Strafe für vorsätzliche Patentverletzung ist Geldstrafe oder Freiheitsstrafe bis zu 3 Jahren, bei Gewerbsmäßigkeit nach Abs. 2 bis zu 5 Jahren. ${ }^{464} \int 25$ GebrMG gilt entsprechend im Gebrauchsmusterrecht und stimmt wörtlich mit $\int 142$ PatG abgesehen von der Definition der objektiven Tatbestände überein.

b. im chinesischen Recht

Nach Art. 58 chin. PatG wird die Straftat im Hinblick auf die strafrechtliche Verantwortlichkeit nach dem Gesetz verfolgt, wenn man das Patent eines anderen für das seine ausgibt und eine Straftat gebildet wird. Vom Gesetz gemeint ist hier Art. 216 des chinesischen Strafgesetzbuchs. Nach Art. 216 chin. StGB kann diese Straftat bis zu drei Jahre Freiheitsstrafe mit Geldstrafe verurteilt.

6. Verjährung

\footnotetext{
${ }^{462}$ Vgl. Ganea, GRURInt. 2002, S. 702.

463 Krasser, S. 930.

464 Benkard, § 142 Rn. 17, S. 1511.
} 
a. im deutschen Recht

Nach SS 141 PatG, 24c GebrMG finden SS 194 ff. BGB entsprechende Anwendung auf die Verjährung der Ansprüche wegen Verletzung des Patentrechts und Gebrauchsmusterrechts.

b. im chinesischen Recht

Für alle Arten von Patentstreitigkeiten ist eine Klagefrist von zwei Jahren geregelt, die von dem Tag an zu rechnen ist, an dem der Patentinhaber von der Verletzung Kenntnis erlangt hat oder hätte erlangen müssen. ${ }^{465}$

In Art. 62 Abs. 2 chin.PatG ist speziell eine Frist für eine Klage wegen Nichtzahlung der in Art. 13 chin. PatG geregelten Vergütung für die Nutzung im Veröffentlichungszeitraum geregelt. Liegt demnach der Zeitpunkt, ab dem von der Verletzung Kenntnis bestand oder hätte bestehen müssen, im Veröffentlichungszeitraum, so ist die zweijährige Klagefrist erst vom Tag der Patenterteilung an zu rechnen. Der Patentinhaber muss somit nicht mehr befürchten, dass die Klagefrist vor der Patenterteilung ausläuft.

\section{Schutz des Markenrechts}

1. Einführung

Nach der Gründung der Volksrepublik China wurden bis heute insgesamt drei markenrechtliche Regelungen erlassen, nämlich die Vorläufige Verordnung über die Markenregistrierung von 1950, die Verordnung über die Verwaltung von Marken von 1963 und das 1982 erlassene Markengesetz. ${ }^{466}$ Das am 1. März 1983 in Kraft getretene Markengesetz 467 wurde dann im Hinblick auf internationale Entwicklungen auf der 30. Sitzung des Ständigen Ausschusses am 22. Februar 1993 revidiert. Das am 1. Juli 1993 in Kraft getretene revidierte Markengesetz dehnte in Übereinstimmung mit dem TRIPS seinen Schutz auf Dienstleistungsmarken aus. 468 Am 27. Oktober 2001 wurde das chin. MarkenG auf der 24. Sitzung des Ständigen Ausschusses des 9. Nationalen Volkskongresses zum zweiten Mal geändert. Außerdem ist die neue Durchführungsverordnung gem. ihres $\int 59$ am 15. September 2002 in Kraft getreten.

Nach \51 chin. MarkenG ist das ausschließliche Nutzungsrecht der eingetragenen Marke begrenzt auf die genehmigte eingetragene Marke und die Waren, für die sie zur Nutzung genehmigt worden ist. Was eine eingetragene Marke anbelangt, ist nach $\int 3$ chin. MarkenG eine durch das Markenamt geprüfte, genehmigte und eingetragene Marke, einschließlich Marken für Waren, Dienstleistungen, Kollektivmarken und Gewährleistungsmarken.

Im deutschen Recht werden Marken, geschäftliche Bezeichnungen und geographische Herkunftsangaben nach $\int 1$ MarkenG geschützt. Das Markenrecht

\footnotetext{
465 Art. 62 chin. PatG

466 Guo, GRURInt. 1997, S. 952.; Siehe dazu zur Geschichte des Markenrechts in der VR China, Bohnet, S. $11 \mathrm{ff}$.

467 Deutsche Übersetzung in: Dietz, S. 359 ff.

468 Guo, GRURInt. 1997, S. 953.
} 
und das Recht an geschäftlichen Bezeichnungen gewähren dem Inhaber ein subjektives und ausschließliches Recht ( $\$ \mathbb{S} 14$ Abs. 1, 15 abs. 1 MarkenG), dessen konkreter Inhalt sich insbesondere aus den $\$ \int ~ 9,11,12$ und 14-19 MarkenG ergibt. ${ }^{469}$ Geographische Herkunftsangaben können zum einen als Kollektivmarke gem. \97 Abs. 1 MarkenG eingetragen werden, aber ein Schutz als Individualmarke ist auch denkbar; Zum anderen können sie Schutz genießen als nicht-eingetragene geographische Herkunftsangabe nach $\ 126$ Abs. 1 MarkenG. ${ }^{470}$

Allerdings spielen Markenrechte 471 beim Technologietransfer nur eine geringe Rolle. Die Marken werden normalerweise gemeinsam mit Übergabe des Patents und Know-how durch den Patentlizenz- und Know-how-Vertrag vom Lizenzgeber an Lizenznehmer lizenziert. Stattdessen werden die Marken genau so gut wie Schutzrechte geschützt.

2. Markenrechtsverletzungen

a. im deutschen Recht

Die SS 14, 15 MarkenG zählen die Handlungen auf, die eine Verletzung des Rechts an einer Marke oder geschäftlichen Bezeichnung darstellen. ${ }^{472}$

i. Identität

Der Markeninhaber genießt zunächst Schutz gegenüber prioritätsjüngeren identischen Marken oder Zeichen, die für identische Waren oder Dienstleistungen geschützt sind oder benutzt werden. ${ }^{473}$ Dies ist ein absoluter Schutz, d.h. die Frage der Verwechslungsgefahr stellt sich bei Identität sowohl der Marken als auch der Waren und Dienstleistungen nicht. ${ }^{474}$

Der Identitätsschutz des $₫ 14$ Abs. 2 Nr. 1 MarkenG betrifft praktisch nur bei der Markenpiraterie gegebenen Fall, dass ein mit der Marke identisches Zeichen für Waren oder Dienstleistungen benutzt wird, die mit denjenigen identisch sind, für die sie Schutz genießt. ${ }^{475}$ Identiät ist nur gegeben, wenn das von einem Dritten benutzte Zeichen in jeder Hinsicht mit dieser geschützten Marke übereinstimmt. ${ }^{476}$

ii. Verwechslungsgefahr

Der Markeninhaber genießt auch Schutz gegenüber prioritätsjüngeren identischen oder ähnlichen Marken oder Zeichen, die für identische oder ähnliche Waren oder Dienstleistungen geschützt sind oder benutzt werden, sofern Verwechslungsgefahr besteht einschließlich der Gefahr, dass die Marken gedanklich miteinander in

\footnotetext{
${ }^{469}$ Vgl. Nordemann, S. 461.

470 Vgl. Nordemann, S. 451.

471 Siehe dazu oben, Erster Teil B IV.

472 Hubmann/Götting, S. 341.

473 §§ 9 Abs. 1 Nr. 1, 14 Abs. 2 Nr. 1 MarkenG

474 Nordemann, S. 380.

475 Vgl. v. Schultz, § 14 Rn. 40. S. 231.

476 v. Schultz, § 14 Rn. 41. S. 231.; Vgl. Ingerl/Ronke, § 14 Rn. 219. S. 564.
} 
Verbindung gebracht werden. ${ }^{477}$ Diese Verwechslungsgefahr muss auf einer Identität oder Ähnlichkeit der sich gegenüberstehenden Zeichen und auf einer Identität oder Ähnlichkeit der sich gegenüberstehenden Waren oder Dienstleistungen beruhen. ${ }^{478}$ Nach der Rechtsprechung des EuGH ist eine Verwechslungsgefahr anzunehmen, wenn die Öffentlichkeit glauben könnte, dass die betreffenden Waren oder Dienstleistungen aus demselben Unternehmen oder jedenfalls aus wirtschaftlich verbundenen Unternehmen stammten. ${ }^{479}$

iii. Schutz bekannter Marken

Schließlich genießt der Markeninhaber Schutz gegenüber prioritätsjüngeren identischen oder ähnlichen Zeichen, die für nicht-ähnliche Waren oder Dienstleistungen eingetragen sind oder benutzt werden, wenn die ältere Marke im Inland bekannt ist und die Benutzung des jüngeren Zeichens die Unterscheidungskraft der älteren Marke beeinträchtigen oder deren Wertschätzung ohne rechtfertigenden Grund in unlauterer Weise ausnutzen würde. 480

Diese Bekanntheitsgrad ist als erreicht anzusehen, wenn die ältere Marke einem bedeutenden Teil des Publikums bekannt ist, das von den durch diese Marke erfassten Waren oder Dienstleistungen betroffen ist, wobei das nationale Gericht bei der Prüfung dieser Voraussetzung alle relevanten Umstände des Falles zu berücksichtigen hat, also insbesondere den Marktanteil der Marke, die Intensität, die geographische Ausdehnung und die Dauer ihrer Benutzung sowie den Umfang der Investitionen, die das Unternehmen zu ihrer Förderung getätigt hat. 481 Die Bekanntheit muss nicht in der Gesamtbevölkerung gegeben sein; vielmehr reicht es aus, wenn diese in einem wesentlichen Teil vorliegt. ${ }^{482}$

iv. Ausdrücklich geregelte unzulässige Benutzungshandlungen

$\int 14$ Abs. 3 beinhaltet nur eine nicht abschließende Aufzählung der wesentlichen Erscheinungsformen aller Zeichenmittel. ${ }^{483}$ Danach sind unzulässige Benutzungshandlungen insbesondere das Anbringen des Zeichens auf Waren, deren Aufmachung oder Verpackung ${ }^{484}$, das Anbieten, In-Verkehr-Bringen oder Besitzen von Waren unter dem Zeichen ${ }^{485}$, das Anbieten oder Erbringen von Dienstleistungen unter dem Zeichen ${ }^{486}$, die Ein- oder Ausfuhr von Waren unter dem Zeichen ${ }^{487}$ einschließlich des Transitweges zwischen zwei Staaten, die nicht der europäischen Gemeinschaft angehören, die Benutzung des Zeichens auf Geschäftspapieren oder in der Werbung ${ }^{488}$.

\footnotetext{
477 § 9 Abs. 1 Nr. 2, 14 Abs. 2 Nr. 2 MarkenG

478 Vgl. v. Schultz, § 14 Rn. 44. S. 232.

479 EuGH GRURInt. 1999, S. 734 ff. - Lloyd; EuGH GRUR 1998, S. 922 ff. - Canon; EuGH GRUR 1998, S. 387 ff. - SABEL/Puma.

480 §§ 9 Abs. 1 Nr. 3, 14 Abs. 2 Nr. 3 MarkenG

481 EuGH GRURInt. 2000, S. 73 ff. - Chevy

482 EuGH GRURInt. 2000, S. 73 f. - Chevy

483 Vgl. v. Schultz, § 14 Rn. 188. S. 298.

$484 \S 14$ Abs. 3 Nr. 1 MarkenG

$485 \S 14$ Abs. 3 Nr. 2 MarkenG

$486 \S 14$ Abs. 3 Nr. 3 MarkenG

$487 \S 14$ Abs. 3 Nr. 4 MarkenG

$488 \S 14$ Abs. 3 Nr. 5 MarkenG
} 
v. Vorbereitungshandlungen

$\int 14$ Abs. 4 MarkenG erfasst die Vorbereitungshandlungen als selbstständige Markenverletzung und betrifft die Verwendung eines identischen oder ähnlichen Zeichens für Aufmachungen, Verpackungen oder Kennzeichnungsmittel wie Etiketten, Anhängern, Aufnähern oder dergleichen, ehe durch deren Verbindung mit Waren oder Dienstleistungen, für die die Marke Schutz genießt, der Tatbestand einer Benutzung des Zeichens für diese Waren oder Dienstleistungen erfüllt wird. ${ }^{489}$

b. im chinesischen Recht

Die Markenrechtsverletzungshandlungen sind in \52 chin. MarkenG aufgezählt. Liegt eines der folgenden Verhalten vor, steht dieses eine Verletzung des ausschließlichen Nutzungsrechts an der eingetragenen Marke dar:

i. Ohne die Erlaubnis des Markeninhabers die Nutzung einer mit seiner eingetragenen Marke identischen oder ähnlichen Marke auf einer identischen oder ähnlichen Art von Waren;

ii. der Vertrieb von Waren, die das ausschließliche Nutzungsrecht an einer eingetragenen Marke verletzen;

iii. das Fälschen oder eigenmächtige Herstellen von Darstellungen eingetragener Marken anderer oder der Verkauf von gefälschten oder eigenmächtig hergestellten Darstellungen eingetragener Marken;

iv. das Ersetzen einer eingetragenen Marke ohne Zustimmung des Markeninhabers und das In-Verkehr-Bringen der mit besagter ersetzten Marke versehenen Waren auf den Markt;

v. das Zufügen eines anderen Schadens für das ausschließliche Nutzungsrecht einer eingetragenen Marke einer anderen Person.

$\int 50$ der Durchführungsverordnung zum chin. MarkenG hat dazu zu diesem Verletzungsverhalten des ausschließlichen Nutzungsrechts an einer eingetragenen Marke genauer erklärt und zwei Verhaltensweisen aufgeführt:

a). Das Benutzen eines mit der eingetragenen Marke anderer identischen oder ähnlichen Kennzeichens als Warenbezeichnung oder Warenausstattung für identische oder ähnliche Waren, wodurch die Öffentlichkeit irregeführt wird;

b). das vorsätzliche Fördern von Verletzungen des ausschließlichen Nutzungsrechts an einer eingetragenen Marke durch Lagerhaltung, Transport, Postsendungen, Verstecken und ähnliche Begünstigungen.

\section{Zivilrechtlicher Schutz}

a. im deutschen Recht

i. Unterlassungsanspruch

Dem Verletzten stehen gegenüber demjenigen, der unbefugt eine Marke oder eine geschäftliche Bezeichnung benutzt, folgende Ansprüche zu: $\iint 14$ Abs. 5, 15 Abs.

${ }^{489}$ Vgl. v. Schultz, § 14 Rn. 213. S. 305. 
4, 128 Abs. 1 MarkenG gewähren dem Rechtsinhaber einen verschuldensunabhängigen $\quad$ Unterlassungsanspruch. $\quad 490 \quad$ Der Unterlassungsanspruch wird ergänzt durch den ebenfalls verschuldensunabhängigen Beseitigungsanspruch, wie er von der Rechtsprechung analog $\int 1004$ BGB entwickelt wurde. ${ }^{491}$

ii. Schadensersatzanspruch

Neben dem Unterlassungsanspruch hat der Inhaber einer Marke oder geschäftlichen Bezeichnung oder geographischer Herkunftsangaben einen markenrechtlichen Schadensersatzanspruch nach $\iint 14$ Abs. 6, 7, 15 Abs. 5, 6, 128 Abs. 2 MarkenG. ${ }^{492}$

iii. Vernichtungsanspruch

$\int 18$ MarkenG gewährt dem Inhaber einer Marke oder einer geschäftlichen Bezeichnung einen zivilrechtlichen Vernichtungsanspruch. Inhaltlich ist $\int 18$ darauf gerichtet, dass widerrechtlich gekennzeichnete Gegenstände ( 18 Abs. 1) oder zur widerrechtlichen Kennzeichnung benutzte Vorrichtungen ( $(18$ Abs. 2) vernichtet und damit dem Wirtschaftskreislauf vollständig und endgültig entzogen werden können. ${ }^{493}$

iv. Auskunftsanspruch

S 19 MarkenG gewährt dem Verletzten den im Falle des Vertriebs kennzeichenverletzender Ware i. S. d. $\iint 14,15$ und 17 bedeutsamen Anspruch auf Drittauskunft, wonach der Verletzer verpflichtet ist, dem Verletzten Auskunft über die Herkunft und den Vertriebsweg von widerrechtlich gekennzeichneten Gegenständen zu erteilen. ${ }^{494}$

b. im chinesischen Recht

i. Unterlassungsanspruch

Nach \57 chin. MarkenG kann der Markeninhaber oder der Inhaber eines ausschließlichen Nutzungsrechts den Erlass einer Anordnung auf Unterlassung beantragen, wenn Beweise für eine stattfindende oder drohende Rechtsverletzung vorgelegt werden und dargelegt wird, dass die rechtmäßigen Rechte und Interessen ohne rechtzeitiges Unterbinden einen schwer wieder gutzumachenden Schaden erleiden würden. ${ }^{495}$

ii. Schadensersatzanspruch

Im Hinblick auf die Geltendmachung von Schadensersatz befindet sich eine detaillierte Regelung im chin. MarkenG. Nach \56 chin. MarkenG bestimmt sich

${ }^{490}$ Hubmann/Götting, S. 341.

491 v. Schultz, § 14 Rn. 240. S. 313.

492 Vgl. Ensthaler, S. 303.

493 v. Schultz, § 18 Rn. 1. S. 363.

${ }^{494}$ Vgl. v. Schultz, § 19 Rn. 1. S. 371.

495 Vgl. Bottenschein, GRURInt. 2005, S. 122. 
die Höhe des Schadensersatzes wegen Verletzung des ausschließlichen Nutzungsrechts einer Marke nach dem Gewinn, den der Verletzer im Verletzungszeitraum durch die Verletzung erlangt hat. Alternativ kann der Schaden geltend gemacht werden, den der Verletzte im Verletzungszeitraum durch die Verletzung erlitten hat, einschließlich der angemessenen Ausgaben, die vom Verletzten zur Unterbindung des rechtsverletzenden Verhaltens aufgewendet worden sind. ${ }^{496}$

Falls die Höhe des Schadens nach einer dieser Berechnungsmethoden schwierig zu ermitteln ist, so bestimmt $\int 56$ Abs. 2 chin. MarkenG, dass der zugesprochene Schadensersatz RMB 500,000 nicht übersteigen darf. Kein Schadensersatzanspruch besteht, wenn der Verkäufer unwissend markenverletzende Waren vertrieben hat und beweisen kann, dass er die Waren selbst legal erworben hat und seinen Lieferanten benennt. ${ }^{497}$

4. Verwaltungsrechtlicher Schutz

Gleich wie beim Patentrecht besteht in China ein doppelspuriges System. Neben der Geltendmachung von Ansprüchen über die Volksgerichte sind bestimmte Behörden für die Durchsetzung gewerblicher Schutzrechte zuständig.

Nach den SS 53 ff. chin. MarkenG bestehen umfassende Befugnisse der Verwaltungsbehörde für Industrie und Handel. ${ }^{498}$ Auf Antrag der Parteien kann die mit der Angelegenheit befasste Verwaltungsbehörde für Industrie und Handel bezüglich der Höhe des Schadensersatzes für die Verletzung des ausschließlichen Nutzungsrechts der Marke eine Schlichtung durchführen. ${ }^{499}$ Außerdem ist diese Behörde auch befugt, Befragungen und Durchsuchungen vorzunehmen und die sofortige Einstellung von Verletzungshandlungen zu verlangen. ${ }^{500}$ Ferner kann die Beschlagnahme und Vernichtung der rechtsverletzenden Waren und zur Fälschung der Darstellung der eingetragenen Marke verwendeten Werkzeuge angeordnet und eine Geldstrafe ausgesprochen werden. ${ }^{501}$

5. Strafrechtlicher Schutz

a. im deutschen Recht

Nach \143 MarkenG ist die vorsätzliche Kennzeichenverletzung strafbar, wenn widerrechtlich im geschäftlichen Verkehr die Verbotstatbestände der $\iint 14,15$ MarkenG verwirklicht sind. ${ }^{502}$ Die Androhung der Freiheitsstrafe von bis zu drei Jahren erhöht sich auf bis zu fünf Jahren bei gewerbsmäßigem Handeln.

Mit einer Freiheitsstrafe bis zu zwei Jahren oder Geldstrafe kann die widerrechtliche Benutzung geographischer Herkunftsangaben ( $\int 127$ MarkenG) nach $\int 144$ MarkenG geahndet werden.

\footnotetext{
$496 \S 56$ Abs. 1 chin. MG

$497 \S 56$ Abs. 3 chin. MG

498 Vgl. Bottenschein, GRURInt. 2005, S. 123.

499 § 53 chin. MG

$500 \S \S 53,55$ Nr 1 chin. MG

$501 \S 53$ chin. MG; Bottenschein, GRURInt. 2005, S. 123.

502 Vgl. Ensthaler, S. 306.
} 
Eine Geldbuße wird nach \ 145 MarkenG gegen denjenigen verhängt, der Hoheitszeichen i. S. v. $\int 8$ Abs. 2 Nr. 6, 7, 8 MarkenG widerrechtlich benutzt oder Mitwirkungs- und Aufklärungspflichten nach \ 134 Abs. 3, 4 MarkenG nicht nachkommt. 503

b. im chinesischen Recht

Nach \59 chin. MarkenG i. V. m. Art. 213, 214, 215 chin. StGB werden drei Markenrechtsverletzungen strafrechtlich verfolgt.

i. Benutzung einer mit einer eingetragenen Marke identischen Marke auf einer identischen Art von Waren ohne Erlaubnis des Markeninhabers;

ii. Das Fälschen oder eigenmächtige Herstellen von Darstellungen eingetragener Marken anderer Personen oder der Vertrieb von gefälschten oder eigenmächtig hergestellten Darstellungen eingetragener Marken sowie

iii. Der wissentliche Vertrieb von Waren mit gefälschten Marken.

Die oben genannten Straftatbestände können mit einer Freiheitsstrafe bis zu 7 Jahren oder Geldstrafe bestraft werden. Berichten zufolge liegen die Geldstrafen in normalen Fällen für natürliche Personen bei ca. 20,000 RMB und für juristische Personen bei ca. 100,000 RMB. ${ }^{504}$ In schwerwiegenden Fällen belaufen sich die Geldstrafen für natürliche Personen auf ca. 100,000 RMB und für juristische Personen auf ca. 500,000 RMB. ${ }^{505}$

6. Verjährung

a. im deutschen Recht

Die Ansprüche aus Rechtsverletzungen verjähren innerhalb von 3 Jahren ab Schluss des Jahres, in das Entstehen des Anspruchs und die Erkenntnis aller Umstände fallen ${ }^{506}$, bei fehlender Kenntnis in 10 bzw. 30 Jahren ab Entstehung bzw. Verletzung ${ }^{507}$.

b. im chinesischen Recht

Die Verjährung der Ansprüche aus Rechtsverletzungen findet keinen Ausdruck im chin. MarkenG. Allerdings ist $\int 135$ chin. AGZR anzuwenden. Die Verjährungsfrist beträgt 2 Jahre, die von dem Tag an zu rechnen ist, an dem der Patentinhaber von der Verletzung Kenntnis erlangt hat oder hätte erlangen müssen. ${ }^{508}$ Nach \ 137 chin. AGZR darf die Verjährungsfrist 20 Jahre ab Entstehung bzw. Verletzung nicht überschreiten.

\section{Schutz des Urheberrechts}

\footnotetext{
503 Vgl. Ensthaler, S. 307.

504 Vgl. Bottenschein, GRURInt. 2005, S. 124.

505 Vgl. Bottenschein, GRURInt. 2005, S. 124.; Pattloch, Intellectual Property in Asia, Chapter 3 China, 2003, S. 78.

506 §§ 199 BGB, 20 MarkenG

507 § 199 Abs. 3 BGB, 20 MarkenG

508 Art. 62 chin. PatG
} 
1. Einführung

Das Urheberrechtsgesetz der VR China vom 7. September $1990^{509}$ wurde durch den am 27. Oktober 2001 auf der 24. Sitzung des Ständigen Ausschusses des IX. Nationalen Volkskongresses gefassten „Beschluss zur Änderung des Urheberrechtsgesetzes der Volksrepublik China“"510 neu gefasst und durch Erlass Nr. 58 des Präsidenten der Volksrepublik China vom 27. Oktober 2001 verkündet. Danach wurde die Durchführungsverordnung zum chin. UrhG ${ }^{511}$ durch Anordnung des Staatsrats der VR China Nr. 359 vom 2. August 2002 erlassen. Gleichzeitig wurde die Vorgänger-Verordnung vom 30. Mai 1991 aufgehoben. Um die Rechte und Interessen der Inhaber des Urheberrechts an Computersoftware zu schützen, wurde die Verordnung über den Schutz von Computersoftware am 20. Dezember 2001512 verkündet. Die bisherige Verordnung über den Schutz von Computersoftware vom 4. Juni 1991 wurde mit Inkrafttreten der neuen Verordnung am 1. Januar 2002 aufgehoben.

In Art. 3 chin. UrhG werden alle einzelnen Werkarten aufgezählt. Dazu gehört auch Computersoftware (Art. 3 Nr. 8 chin. UrhG). Unter Computersoftware im Sinne der chin. Computersoftware-VO sind Computerprogramme und die dazugehörige Dokumentation zu verstehen. „Computerprogramme“ sind nach $\ 3$ chin. Computersoftware-VO codierte Befehlsfolgen zur Erreichung eines bestimmten Ergebnisses, die durch Computer und dgl. zur Informationsverarbeitung geeignete Anlagen ausgeführt werden, oder symbolische Befehls- oder Satzfolgen, die automatisch in codierte Befehlsfolgen umgewandelt werden können. Quell- und Objektcode desselben Computerprogramms gelten als ein und dasselbe Werk. Unter „Dokumentation“ sind zur Darstellung von Inhalt, Struktur, Gestaltung, Funktionsstandards, Entwicklungsstand, Testergebnissen und Nutzungsmethoden eines Programms verwendete Textmaterialien, graphische Darstellungen und dgl. zu verstehen, wie etwa Beschreibungen der Programmgestaltung, Flussdiagramme oder Nutzerhandbücher.

Im deutschen Recht sind die Computerprogramme mit der 1985 erfolgten Novellierung des UrhG den Sprachwerken zugeordnet bzw. diesen gleichgestellt worden. ${ }^{513}$ Denn sie teilen in einer Symbolsprache, die dem Computer verständlich ist (sog. Quellcode oder Sourcecode), die Lösung von technischen Problemen mit. ${ }^{514}$ Mit den $\$ \int 69$ a ff. des UrhG wurde die EG-Richtlinie über den Rechtsschutz von Computerprogrammen aus dem Jahre 1991 in deutsches Recht umgesetzt. ${ }^{515}$ \69a bestimmt den Schutz für sämtliche Ausdrucksformen von Computerprogrammen, also insbes. auch für Quellen- und Maschinencodes. ${ }^{516}$ Sie werden geschützt, wenn sie individuelle Werke in dem Sinne darstellen, dass sie

\footnotetext{
509 Deutsche Übersetzung, GRURInt. 1990, S. 940 ff.; Siehe dazu zum chin. UrhG 1990, Thaler, S. 136 ff.

510 Deutsche Übersetzung, GRURInt. 2002, S. 23 ff.

511 Deutsche Übersetzung, GRURInt. 2003, S. $1008 \mathrm{ff}$.

512 Deutsche Übersetzung, GRURInt. 2003, S. 47 ff.

513 Vgl. Ensthaler, S. 7.

${ }^{514}$ Rehbinder, S. 68.

515 Richtlinie über den Rechtsschutz von Computerprogrammen, ABl. L der EG Nr. 1122/42.

${ }^{516}$ Ensthaler, S. 52.
} 
das Ergebnis der eigenen geistigen Schöpfung ihres Urhebers sind.

Abgesehen von den Computerprogrammen spielt das Urheberrecht beim Technologietransfer nur eine geringe Rolle. ${ }^{517}$ Deswegen wird damit viel Wert auf die Besonderheiten der Computerprogramme gelegt.

2. Urheberrechtsverletzungen

a. im deutschen Recht

i. Verwertungsverbot

Der Urheber wird durch $\ 96$ UrhG dagegen geschützt, dass ein Werknutzer, der sich weder als Täter noch als Teilnehmer an der rechtswidrigen Herstellung von Vervielfältigungsstücken des Werkes beteiligt hat, diese Vervielfältigungsstücke frei verwerten kann. ${ }^{518}$ \96 UrhG ist keine Anspruchsgrundlage; Ist der Tatbestand

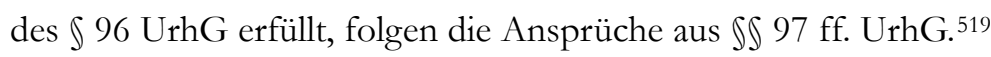

\section{ii. Schutzgegenstand}

Nach $\int 97$ Abs. 1 S. 1 UrhG sind das Urheberrecht oder ein anderes nach diesem Gesetz geschütztes Recht. Gemeint sind damit nur absolute Rechte. ${ }^{520}$ Dazu gehören die aus dem Urheberpersönlichkeitsrecht folgenden Befugnisse, nämlich das Veröffentlichungsrecht ( $(12)$, das Recht auf Anerkennung der Urheberschaft (\$13) und das Recht, die Entstellung des Werkes zu verbieten (\$ 14); Verwertungsrechte der Urheber ( $\$ \int$ 15-24) sowie Zustimmungsrechte der Urheber $(\mathbb{S} 23,34,35) .521$

iii. Aktivlegitimation

Wird das Persönlichkeitsrecht des Urhebers oder Inhabers eines verwandten Schutzrechts verletzt, liegt die Aktivlegitimation bis zu seinem Tode bei ihm selbst. ${ }^{522}$ Stirbt der Urheber bzw. der nach $\int S 70,72$ Berechtigte, sind seine Rechtsnachfolger i. S. v. \\$ 28, 29 aktivlegitimiert.

Bei den ausschließlichen Nutzungsrechten des Urhebers bzw. der Schutzrechtsinhaber ist aktivlegitimiert, wer Inhaber der Rechte ist, also auch der ausschließliche Lizenznehmer als Inhaber der Verwertungsrechte. ${ }^{523}$ Dagegen kann der einfache Lizenznehmer grundsätzlich nicht aus eigenem Recht klagen. ${ }^{524}$

iv. Passivlegitimation

Urheberrechtsverletzungen und Verletzungen verwandter Schutzrechte sind wie alle Verletzungen von Rechten an immateriellen Gütern unerlaubte Handlungen

\footnotetext{
517 Siehe dazu oben Erster Teil B V.

518 Wandtke/Bullinger, § 96 Rn. 1. S. 1071.

519 Dreyer/Kotthoff/Meckel, § 96 Rn. 1. S. 1063.

520 Vgl. Schricker, § 97 Rn. 2a. S. 1878.; Dreyer/Kotthoff/Meckel, § 97 Rn. 1. S. 1069.; Wandtke/Bullinger, § 97 Rn. 2. S. 1096.

${ }^{521}$ Vgl. Fromm/Nordemann, § 97 Rn. 2 ff. S. 642.; Dreyer/Kotthoff/Meckel, § 97 Rn. 1 ff. S. 1069 f.

${ }^{522}$ Dreyer/Kotthoff/Meckel, § 97 Rn. 6. S. 1072.

523 BGH GRUR 1995, S. 338 ff. - Kleiderbügel; Vgl. Schricker, § 97 Rn. 28. S. 1889.

524 Vgl. Schricker, § 97 Rn. 30. S. 1891.
} 
im Sinne der $\iint 823$ ff. BGB 525

v. Widerrechtlichkeit der Verletzung

Voraussetzung für den Anwendungsbereich der $\iint 97$ ff. ist ein Eingriff in die oben genannten absoluten Rechte des Urhebers. Ob ein Eingriff in geschützte Rechte vorliegt, bestimmt sich nach den einschlägigen Regelungen. Der Eingriff darf auch nicht gerechtfertigt sein. Ein Rechtfertigungsgrund liegt vor, wenn der Rechtsinhaber in die Benutzungshandlung eingewilligt oder sie genehmigt hat. ${ }^{526}$ Als weitere Rechtfertigungsgründe kommen in Betracht: Notwehr und Notstand (\$S 227, 228 BGB), die erlaubte Selbsthilfe (\$S 229 ff. BGB) und der sog. Übergesetzliche Notstand. ${ }^{527}$

vi. Straftatbestände

SS 106-108 und $\int 108$ b Abs. 1 und 2 UrhG enthalten materiellrechtliche Straftatbestände, die bestimmte Handlungen bestrafen. Als strafbare Tathandlungen erwähnt die Vorschrift unter bestimmten Voraussetzungen das „Vervielfältigen“, „Verbreiten“ oder die „öffentliche Wiedergabe“ des urheberrechtlich geschützten Werks. 528 SS 107, 108 schützen das Urheberpersönlichkeitsrecht und die Verwertungsrechte der Inhaber verwandter Schutzrechte.

b. im chinesischen Recht

Die urheberrechtsverletzenden Handlungen sind im chin. UrhG aufgeführt. Ist einer der elf in Art. 46 chin. UrhG aufgeführten Tatbestände erfüllt, so hat der Verletzer die zivilrechtliche Haftung zu übernehmen. Außer zivilrechtlicher Haftung können die Rechtsverletzungshandlungen in Art. 47 UrhG einen Verstoß gegen das Strafrecht darstellen.

Abgesehen von den Vorschriften chin. UrhG werden die Rechtsverletzungshandlungen in $\ 23$ chin. Computersoftware-VO aufgezählt, die nur zivilrechtliche Haftung verursachen.

i. Wer Software ohne Erlaubnis des Inhabers des Softwareurheberrechts veröffentlicht oder registriert;

ii. Wer die Software eines anderen als seine eigene Software veröffentlicht oder registriert;

iii. Wer ohne Erlaubnis der Mitentwickler in Zusammenarbeit entwickelte Software als von ihm alleine fertig gestellte Software veröffentlicht oder registriert; iv. Wer auf der Software eines anderen mit dem eigenen Namen zeichnet oder den Namen, mit dem die Software eines anderen bezeichnet ist, ändert;

v. Wer Software ohne Zustimmung des Inhabers des Softwareurheberrechts ändert oder übersetzt;

vi. Wer sonstige das Softwareurheberrecht verletzende Handlungen begeht.

\footnotetext{
${ }^{525}$ Schricker, § 97 Rn. 35. S. 1893.

526 Vgl. Wandtke/bullinger, § 97 Rn. 30. S. 1104.; Dreyer/Kotthoff/Meckel, § 97 Rn. 15. S. 1076.

${ }^{527}$ Dreyer/Kotthoff/Meckel, § 97 Rn. 16. S. 1076.

528 Schricker, § 106 Rn. 4. S. 1991.
} 
$\int 24$ chin. Computersoftware-VO enthält Tatbestände, die Art. 47 chin. UrhG entsprechend strafrechtliche Haftung herbeiführen können.

i. Wer die Software eines Urheberrechtsinhabers vervielfältigt oder teilweise vervielfältigt;

ii. Wer die Software eines Urheberrechtsinhabers an die Öffentlichkeit verbreitet, vermietet oder durch Informationsnetze überträgt;

iii. Wer vorsätzlich die von einem Urheberrechtsinhaber zum Schutz seines Softwareurheberrechts getroffenen technischen Vorkehrungen umgeht oder zerstört;

iv. Wer vorsätzlich elektronische Informationen für die Wahrnehmung von Rechten an Software beseitigt oder verändert;

v. Wer das Softwareurheberrecht des Urheberrechtsinhabers überträgt oder Dritten zur Ausübung einräumt.

3. Zivilrechtlicher Schutz

a. im deutschen Recht

i. Beseitigungsanspruch

Wer durch einen rechtswidrigen Eingriff einen fortdauernden störenden Zustand geschaffen hat, muss diesen nach $\int 97$ Abs. 1 S. 1 beseitigen. ${ }^{529}$

ii. Unterlassungsanspruch

\ 97 Abs. 1 S. 1 gibt dem Verletzten nach begangener Verletzungshandlung einen Anspruch auf Unterlassung, wenn eine Wiederholungsgefahr besteht. ${ }^{530}$

iii. Schadensersatzanspruch

Schadensersatzansprüche nach \97 Abs. 1 S. 1 setzen Verschulden, d. h. Vorsatz oder Fahrlässigkeit voraus. Der schuldhafte Eingriff in absolute Rechte zum Schadensersatz verpflichtet. 531

iv. Herausgabe des Gewinns und Auskunfts- und Rechnungslegungsanspruch $\int 97$ Abs. 1 S. 2 gibt dem Verletzten das Recht, an Stelle des Schadensersatzes die Herausgabe des Gewinns zu verlangen, den der Verletzer durch die Verletzung des Rechts erzielt hat. ${ }^{532}$ Der Verletzte kann zur Vorbereitung eines bezifferten Schadensersatz- oder Bereicherungsanspruchs vom Verletzer Auskunft und Rechnungslegung verlangen. ${ }^{533}$

v. Vernichtung- und Überlassungsanspruch

\footnotetext{
529 Vgl. Schricker, § 97 Rn. 45. S. 1911.

530 Dreyer/Kotthoff/Meckel, § 97 Rn. 19. S. 1078.

531 Vgl. Schricker, § 97 Rn. 50. S. 1913.

532 Dreyer/Kotthoff/Meckel, § 97 Rn. 30. S. 1084.

533 Schricker, § 97 Rn. 81. S. 1935.
} 
Gegenstand der Vernichtung nach $₫ 98$ Abs. 1 sind in erster Linie alle rechtswidrig hergestellten, rechtswidrig verbreiteten oder zur rechtswidrigen Verbreitung bestimmten Vervielfältigungsstücke, die im Besitz oder Eigentum des Verletzers stehen. Gegenstand der Beseitigungsmaßnahmen gem. \99 sind außerdem ausschließlich oder nahezu ausschließlich zur rechtswidrigen Herstellung von Vervielfältigungsstücken bestimmte Vorrichtungen im Eigentum des Verletzers.

Statt der Maßnahmen der $\iint 98$ Abs. 1, 99 kann der Verletzte Überlassung der Vervielfältigungsstücke oder Vorrichtungen gegen Entgelt verlangen ( $\$ S 98$ Abs. 2, 99).534 \69f UrhG erweitert die Ansprüche aus $\int \mathbb{9} 98$ und 99 insofern, als der Eigentümer oder Besitzer der Vervielfältigungsstücke nicht der Verletzer zu sein braucht. ${ }^{535}$

b. im chinesischen Recht

i. Anspruchsgrundlage nach Art. 46 ff. chin. UrhG

Die zivilrechtlichen Schutzmöglichkeiten sind in Art. 46, 47 chin. UrhG aufgeführt. Entsprechend den Umständen ist die zivilrechtliche Haftung zu übernehmen durch:

a). Einstellung der Rechtsverletzung;

b). Beseitigung ihrer Wirkungen;

c). Bitte um Entschuldigung;

d). Leistung von Schadensersatz, etc.

Die Rechnungsmethode des Schadensersatzes ist in Art. 48 chin. UrhG geregelt. Die Maßgabe ist zunächst der konkrete Schaden des Rechtsinhabers. Der rechtswidrige Gewinn des Verletzers wird dann eingezogen, wenn der konkrete Schaden nur schwer berechnet werden kann. Im Schadensersatzbetrag sind die angemessenen Kosten umgefasst, die der Rechtsinhaber verauslagt hat, um die rechtsverletzende Handlungen zu verhindern.

ii. Anspruchsgrundlage nach $\$ \int 23,24$ chin. Computersoftware-VO

Die gleiche zivilrechtliche Haftung wie in Art. 46, 47 chin. UrhG ist auch in $\$ \$ 23$, 24 chin. Computersoftware-VO zu finden. Nach $₫ 25$ chin. Computersoftware-VO findet Art. 48 chin. UrhG auch Anwendung auf die Höhe des Schadensersatzes für die Verletzungen des Softwareurheberrechts.

In Art. 28 chin. Computersoftware-VO ist der Beweisumkehr für den Verleger, Hersteller, Verbreiter und Vermieter von Software-Vervielfältigungsstücken geregelt. Danach müssen sie dafür haften, wenn sie nicht beweisen können, dass sie die Vervielfältigungsstücke aus rechtmäßiger Quelle erhalten haben.

Hat ein Besitzer von Software-Vervielfältigungsstücken kein Nutzungsrecht und besteht auch kein triftiger Grund dafür, dass er hätte wissen müssen, dass die betreffende Software eine Urheberrechtsverletzung darstellt, so haftet er nach $₫ 30$

\footnotetext{
534 Schricker, § 98/99, Rn. 9. S. 1955.

535 Schricker, § 69f Rn. 3. S. 1391.
} 
chin. Computersoftware-VO zwar nicht für Schadensersatz, muss jedoch die Nutzung einstellen und die verletzenden Vervielfältigungsstücke vernichten. Ist die Einstellung und Vernichtung der verletzenden Vervielfältigungsstücke für den Nutzer nur unangemessen, so kann dieser die Nutzung nach Zahlung einer angemessenen Gebühr an den Softwareurheberrechtsinhaber fortführen.

4. Verwaltungsrechtlicher Schutz

In Art. 47 chin. UrhG wird auch verwaltungsrechtlicher Rechtsschutz geregelt. Wenn eine Rechtsverletzungshandlung gleichzeitig öffentliche Interessen verletzt, so können durch die Urheberrechtsverwaltungsbehörden die Einstellung der Rechtsverletzungshandlung, die Einziehung des rechtswidrigen Gewinns, die Einziehung und Vernichtung der rechtsverletzenden Vervielfältigungsstücke angeordnet werden, auch kann eine Geldbuße auferlegt werden. Bei erschwerten Umständen können die Urheberrechtsverwaltungsbehörden auch die vorwiegend zur Herstellung der rechtsverletzenden Vervielfältigungsstücke verwendeten Materialien, Werkzeuge und Vorrichtungen etc. einziehen. \24 chin. Computersoftware-VO hat auch die gleiche Regelung.

Ist eine Partei mit einer Verwaltungsstrafsanktion nicht einverstanden, so kann sie nach Art. 55 chin. UrhG innerhalb von 3 Monaten seit dem Tag, an dem sie das Schriftstück mit der Entscheidung über die Verwaltungsstrafsanktion erhalten hat, beim Volksgericht Klage erheben.

\section{Strafrechtlicher Schutz}

a. im deutschen Recht

Die SS 106 ff. enthalten das Urheberstrafrecht. Hat der Täter den objektiven Tatbestand durch die Vervielfältigung, die Verbreitung oder die öffentliche Wiedergabe eines Werks oder der Bearbeitung oder Umgestaltung eines Werks verwirklicht, vorsätzlich, rechtswidrig und schuldhaft gehandelt, so kommt eine Freiheitsstrafe von bis zu drei Jahren oder eine Geldstrafe in Betracht. ${ }^{536}$

Der Tatbestand des $\ 108$ a setzt die Erfüllung eines Tatbestandes der $\$ \rrbracket 106$ bis 108 voraus. Als einziges weiteres Tatbestandsmerkmal fordert $\ 108$ a ein gewerbsmäßiges Verhalten des Täters. Liegt ein Fall der gewerbsmäßigen Verletzung von Urheber- oder Leistungsschutzrechten vor, sieht $\ 108$ a einen Strafrahmen von bis zu fünf Jahren vor.

b. im chinesischen Recht

Die strafrechtliche Haftung ist in Art. 47 chin. UrhG, \ 24 chin. Computersoftware-VO i. V. m. Art. 217, 218 chin. StGB über die Straftaten der Urheberrechtsverletzung und des Verkaufs verletzender Vervielfältigungsstücke in gesetzmäßiger Weise zu ermitteln. Liegt eine Straftat der Urheberrechtsverletzung nach Art. 217 chin. StGB vor, um den Gewinn zu erzielen und der Betrag des rechtswidrigen Gewinns relativ groß ist oder andere schweren Umstände vorliegen,

${ }^{536}$ Vgl. Dreyer/Kotthoff/Meckel, § 106 Rn. 9. S. 1111. 
so kann eine Freiheitsstrafe bis zu drei Jahren oder zusammen mit einer Geldstrafe oder allein eine Geldstrafe verhängt werden; ist der Betrag des rechtswidrigen Gewinns sehr groß oder liegen andere besonders schweren Umstände vor, so kann eine Freiheitsstrafe von drei bis zu sieben Jahren zusammen mit einer Geldstrafe verhängt werden.

\section{Verjährung}

a. im deutschen Recht

Gem. \102 UrhG verjähren Ansprüche wegen Urheberrechtsverletzungen übereinstimmend mit $\$ 195$ BGB in drei Jahren.

b. im chinesischen Recht

Die Verjährung der Ansprüche aus Rechtsverletzungen findet keinen Ausdruck im chin. UrhG. Allerdings ist $\ 135$ chin. AGZR anzuwenden. Die Verjährungsfrist beträgt 2 Jahre, die von dem Tag an zu rechnen ist, an dem der Patentinhaber von der Verletzung Kenntnis erlangt hat oder hätte erlangen müssen. ${ }^{537}$ Nach $\ 137$ chin. AGZR darf die Verjährungsfrist 20 Jahre ab Entstehung bzw. Verletzung nicht überschreiten.

\section{Der Schutz des Know-how ${ }^{538}$}

\section{I. im deutschen Recht}

1. Der zivilrechtliche Schutz

\section{a. Geheimnisverrat}

Beim zivilrechtlichen Schutz von Geschäfts- und Betriebsgeheimnissen geht es darum, dass jemand eine seinem Willen zuwiderlaufende Weitergabe oder Verwertung bestimmter Kenntnisse, Erfahrungen und Informationen verhindern will oder Ersatz des ihm aus einer solchen Weitergabe oder Verwertung erwachsenen Schadens begeht. ${ }^{539} \mathrm{Zu}$ diesem Zweck enthält die Vorschrift zwei sorgfältig zu unterscheidende Tatbestände. Der erste betrifft nur den Geheimnisverrat durch bei einem Unternehmen beschäftigte Personen während der Geltungsdauer ihres Dienstverhältnisses( $\left(17\right.$ Abs. 1 UWG). ${ }^{540}$ Der zweite Tatbestand ( $(17$ Abs. 2 UWG) wendet sich dagegen zwar an jedermann (einschließlich der Beschäftigten eines Geschäftsbetriebes), erfasst dafür jedoch nur einzelne, besonders qualifizierte Fälle der Ausspähung541 oder Verwertung

\footnotetext{
537 Art. 62 chin. PatG

538 Siehe dazu oben, Erster Teil B VI.

539 Vgl. Krasser, GRUR 1977, S. 177.; Emmerich, Unlauterer Wettbewerb, S. 168 f.

540 Siehe dazu zum Geheimnisverrat durch Beschäftigte, Emmerich, Unlauterer Wettbewerb, S. 167 ff.; Harte-Bavendamm/Henning-Bodewig, § 17, Rn. 8 ff. S. 2111 ff.; Fezer, UWG, § 17 Rn. 26 ff. S. 904 ff.; Hefermehl/Köhler/Bornkamm, § 17 Rn. 4 ff. S. 1231 ff.

${ }^{541}$ Siehe dazu zur Ausspähung, Hefermehl/Köhler/Bornkamm, § 17 Rn. 29 ff. S. 1235 ff.;
} 
fremder Geschäfts- oder Betriebsgeheimnisse ${ }^{542} .543$

Denn die Strafvorschriften der $\iint 17$ UWG bilden Schutznormen im Sinne des \ 823 Abs. 2 BGB, so hat der Verletzer der Geheimnisse dem Geheimnisinhaber Schadensersatz $\mathrm{zu}$ leisten. Nach den auch im Anwendungsbereich des $₫ 823$ Abs. 2 BGB seit langem anerkannten Grundsätzen des Deliktsrechts aus dem Rechtsgedanken der \$S 12, 862, 1004 BGB: gegen eine ernsthaft drohende rechtswidrige Beeinträchtigung seiner durch $\int 17$ UWG geschützten Interessen kann sich der Geheimnisinhaber mit der Unterlassungsklage wehren. ${ }^{544}$

b. Vorlagenfreibeuterei nach $₫ 18 \mathrm{UWG}$

Vorlagen sind Mittel, die als Grundlage oder Vorbild für die Herstellung von neuen Sachen oder Dienstleistung dienen sollen. ${ }^{545}$ Die Anwendung des $\ 18$ Abs. 1 UWG setzt voraus, dass die fraglichen Vorlagen oder Vorschriften dem Täter im geschäftlichen Verkehr anvertraut worden sind. ${ }^{546}$ Die strafbare Handlung besteht in der unbefugten Verwertung oder Mitteilung der anvertrauten Vorlagen zu Zwecken des Wettbewerbs oder aus Eigennutz. ${ }^{547}$

\18 stellt eine wichtige Schutzvorschrift gegenüber Geschäftspartnern dar, denen etwa als Lieferanten, Subunternehmen oder Zulieferern zur Erfüllung des Auftrags geheimes Know-how überlassen werden muss. ${ }^{548}$

Jeder Verstoß gegen $\ 18$ kann wie bei $\ 17$ zivilrechtlich nach $₫ 823$ Abs. 2 BGB verfolgt werden und entsprechende Ansprüche begründen.

\section{Der strafrechtliche Schutz}

Bei der Durchsetzung des UWG spielen heute die strafrechtlichen Sanktionen neben den zivilrechtlichen nur noch eine untergeordnete Rolle. ${ }^{549}$ Nach \ 17 UWG wird eine Straftat zum Geheimnisverrat mit Freiheitsstrafe bis zu drei Jahren oder mit Geldstrafe bestraft. Mit Freiheitsstrafe bis zu zwei Jahren oder mit Geldstrafe wird Vorlagenfreibeuterei nach $₫ 18$ UWG bestraft. Für die beiden ist der Versuch strafbar.

\section{II. im chinesischen Recht}

Als Geschäftsgeheimnisse werden in $\ 10$ Abs. 3 des „Gesetz der VR China gegen den unlauteren Wettbewerb“550 technische Daten und Daten zur Betriebsführung bezeichnet, die nicht allgemein bekannt sind, dem Berechtigten wirtschaftlichen Nutzen bringen können, praktisch brauchbar sind, und die geheim zu halten der

\footnotetext{
Harte-Bavendamm/Henning-Bodewig, § 17 Rn. 18 ff. S. 2114 ff.; Fezer, § 17 Rn. 50 ff. S. 908 ff.

542 Siehe dazu zur Geheimnisverwertung, Hefermehl/Köhler/Bornkamm, § 17 Rn. 40 ff. S. 1236 ff.;

Harte-Bavendamm/Henning-Bodewig, § 17 Rn.26 ff. S. 2116 ff.; Fezer, § 17 Rn. 64 ff. S. 911 ff.

543 Emmerich, Unlauterer Wettbewerb, S. 167.

544 Vgl. Krasser, GRUR 1977, S. 178.

545 Hefermehl/Köhler/Bornkamm, § 18, Rn. 9. S. 1244.; Vgl. Emmerich, Unlauterer Wettbewerb, S. 175.

546 Emmerich, Unlauterer Wettbewerb, S. 175.

547 Emmerich, Unlauterer Wettbewerb, S. 176.

548 Fezer, UWG, § 18, Rn. 4 S. 917.

549 Emmerich, Unlauterer Wettbewerb, S. 504.

550 Verabschiedet am 2. September 1993 auf der 3. Sitzung des 8. Nationalen Volkskongresses; deutsche Übersetzung in: GRURInt. 1994, S. 1001 ff.
} 
Berechtigte Maßnahmen ergriffen hat.

\section{Eingriffstatbestand}

Die Geschäftsgeheimnisse verletzenden Handlungen sind in $\ 10$ chin. UWG aufgezählt. Sie werden durch die Unternehmer dadurch verletzt, dass sie

a. mit Diebstahl, Inaussichtstellen eines Vorteils, Drohung oder anderen unlauteren Methoden Geschäftsgeheimnisse des Berechtigten erlangen;

b. Geschäftsgeheimnisse des Berechtigten, die mit den Methoden der vorigen Nummer erlangt wurden, bekannt werden lassen, gebrauchen oder ihren Gebrauch durch andere gestatten;

c. in Verletzung von Vereinbarungen oder der Forderung des Berechtigten, Geschäftsgeheimnisse zu wahren, die Geschäftsgeheimnisse in seiner Hand bekannt werden lassen, gebrauchen oder ihren Gebrauch durch andere gestatten.

\10 Abs. 2 chin. UWG gilt auch für Dritte, der von rechtswidrigen Handlungen weiß oder wissen muss und Geschäftsgeheimnisse anderer erlangt, gebraucht oder bekannt werden lässt.

\section{Rechtsfolge}

Nach \25 chin. UWG geben die Überwachungs- und Prüfungsbehörden Anweisung, die rechtswidrige Handlung einzustellen, und können je nach den Umständen Geldbußen in Höhe von 10, 000 bis 100,000 Yuan verhängen, wenn einer der Tatbestände nach $\$ 10$ chin. UWG vorliegt.

Art. 219 chin. StGB enthält die gleichen Tatbestände wie $₫ 10$ chin. UWG. Der einzige Unterschied liegt darin, dass der Straftatbestand den schweren Schaden des Geheimnisinhabers voraussetzt. Dem Verletzer wird eine Freiheitsstrafe bis zu drei Jahren zusammen mit einer Geldstrafe oder eine Geldstrafe allein verhängt; ist der Schaden besonders schwer, wird eine Freiheitsstrafe von drei bis zu sieben Jahren mit einer Geldstrafe verhängt.

\section{Rechtsdurchsetzung zum Schutz des geistigen}

\section{Eigentums}

\section{Vorläufige Schutzmaßnahmen}

1. im deutschen Recht

In dringlichen Fällen kann der Unterlassungsanspruch im Wege der einstweiligen Verfügung ( $\$ \$$ 935, 940 ZPO) geltend gemacht werden. 551 Eine einstweilige Verfügung stellt stets nur eine vorläufige Maßnahme dar, die nicht zu einer rechtskräftigen Feststellung des Anspruchs i. S. d. \197 Abs. 1 Nr. 3 BGB führt. 552

\footnotetext{
551 Vgl. v. Schultz, § 14 Rn. 268. S. 325.; Siehe dazu zur einstweiligen Verfügung, Krasser, S. 918 ff.

552 v. Schultz, § 14 Rn. 275. S. 327.
} 
2. im chinesischen Recht

Die Institution der einstweiligen Verfügung ist in den $\iint 74,92$ bis 99 chin. ZPG geregelt, nämlich, die Beweissicherung, die Vermögenssicherung und die Vorwegvollstreckung.

\section{a. Beweissicherung}

Nach \ 74 chin. ZPG, \58 Abs. 1 chin. MG und \50 Abs. 1 chin. UrhG können der Markeninhaber oder ein sonst Interessierter bei der Unterbindung eines rechtsverletzenden Verhaltens im Falle, dass möglicherweise Beweise verloren gehen oder später schwer zu erlangen sind, vor Klageerhebung vor den Volksgerichten Beweissicherung beantragen.

b. Vermögenssicherung

Nach \92 Abs. 1 chin. ZPG kann das Gericht in Fällen, in denen Handlungen einer Partei oder andere Gründe dazu führen können, dass sich ein Urteil nicht oder nur schwer vollstrecken lässt, auf Antrag der klagenden Partei, nötigenfalls auch von Amts wegen, gegenüber der beklagten Partei Vermögenssicherung anordnen. Das Gericht kann gem. \92 Abs. 2 chin. ZPG selbst darüber entscheiden, ob der Antragssteller Sicherheit leisten muss.

Nach Art. 61 chin. PatG, \57 chin. MG und \ 49 chin. UrhG i. V. m. \ 93 Abs. 1 chin. ZPG kann die Vermögenssicherung beim Gericht schon vor der Klageerhebung beantragt werden, wenn sich der Antragssteller in der Zwangslage befindet, dass er ohne die sofortige Anordnung der Vermögenssicherung einen nur schwer wieder gutzumachenden Schaden erleiden würde. Für die vorprozessuale Vermögenssicherung muss der Antragssteller Sicherheit leisten; tut er dies nicht, so wird der Antrag nach $\int 93$ Abs. 1 Satz 2 chin. ZPG zurückgewiesen. Nach \93 Abs. 2 chin. ZPG muss das Gericht innerhalb von 48 Stunden nach Erhalt des Antrags eine Anordnung treffen und danach muss die Vollsteckung sofort beginnen.

c. Vorwegvollstreckung

Nach $\int 97$ Abs. 1 Fall 3 chin. ZPG kann das Gericht auf Antrag der klagenden Partei eine Vorwegvollstreckung gegen die beklagte Partei nur dann anordnen, wenn diese wegen Dringlichkeit erforderlich ist. Die Dringlichkeit ist nach Meinung des Obersten Volksgerichts gegeben, wenn die sofortige Einstellung von Verletzungen, die sofortige Beseitigung von Behinderungen oder die sofortige Unterlassung einer bestimmten Handlung erforderlich sind. ${ }^{553}$

Nach \98 Abs. 1 chin. ZPG ist die Anordnung der Vorwegvollstreckung an zwei Voraussetzungen gebunden: Zum einen müssen die Rechte- und Pflichtenbeziehungen zwischen den Parteien klar sein, auch muss das persönliche Leben oder die Produktions- und Gewerbetätigkeit des Antragsstellers erheblich

553 Liu, GRURInt. 1999, S. 489.; Vgl. Punkt 107 der „Ansichten des Obersten Volksgerichts zu einigen Fragen der Anwendung des Zivilprozeßgesetzes (1992)“, Zuigao Renmin Fayuan Gongbao (Amtsblatt des Obersten Volksgerichts) 1992 Nr. 3, S. 70, S. 78. 
beeinträchtigt sein, wenn nicht vorweg vollstreckt wird; Zum zweiten muss der Antragsgegner in der Lage sein, das Klageverlangen zu erfüllen.

\section{Grenzbeschlagnahme durch den Zoll}

1. im deutschen Recht

a. Beim Patentrecht und Gebrauchsmusterrecht

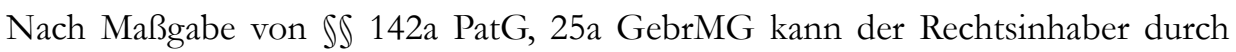
einen bei der Oberfinanzdirektion zu stellenden Antrag erreichen, dass die Zollbehörde schutzrechtsverletzende Erzeugnisse bei deren Ein- oder Ausfuhr beschlagnahmt, wenn die Rechtsverletzung offensichtlich ist. ${ }^{554}$

b. Beim Markenrecht

Das Verfahren bezüglich der Beschlagnahme von Waren bei der Einfuhr und Ausfuhr wird in den $\int S 146$ bis 151 MarkenG geregelt. Ist die Einfuhr zeichenverletzender Ware zu besorgen, kann ein sog. „Grenzbeschlagnahmeantrag“ nach $₫ 148$ Abs. 1 MarkenG gestellt werden. ${ }^{555}$ Die Zollbehörden kontrollieren und beschlagnahmen nach bestimmten Gesichtspunkten verdächtige Ware beim Grenzeintritt in die Bundesrepublik Deutschland, sofern nach ihrer Prüfung eine offensichtliche Rechtsverletzung vorliegt ( $(146$ Abs. 1 Satz 1 MarkenG). Grenzbeschlagnahmeanträge für Deutschland sind zu stellen bei der Oberfinanzdirektion; sie sind jeweils für 2 Jahre gültig (\$148 Abs. 1 MarkenG).

c. Beim Urheberrecht

Vervielfältigungsstücke, deren Herstellung oder Verbreitung ein Urheber- oder Leistungsschutzrecht verletzt, unterliegen auf Antrag des Rechtsinhabers bei ihrer Einfuhr oder Ausfuhr der Beschlagnahme durch die Zollbehörden, sofern die Rechtsverletzung offensichtlich ist und der Rechtsinhaber Sicherheit leistet $(\mathbb{\Omega} 111 \mathrm{~b}$ Abs. 1 Satz 1 UrhG). ${ }^{556}$

2. im chinesischen Recht

a. Einführung

Das Grenzbeschlagnahmeverfahren in China ist seit dem 1. März 2004 neu geregelt durch vom Staatsrat erlassene Bestimmungen zum Zollschutz des geistigen Eigentums ${ }^{557}$. Nach $\ 2$ der chin. Zollbestimmungen wird das unter dem Zollschutz stehende geistige Eigentum als das Ausschließlichkeit der Marken, Urheberrecht und Verwandte Schutzrechte sowie Patentrecht definiert. Verboten

\footnotetext{
${ }^{554}$ Krasser, S. 920.

555 Vgl. Nordemann, S. 464.

${ }^{556}$ Rehbinder, S. 432.; Siehe dazu zur Grenzbeschlagnahme, Dreyer/Kotthoff/Meckel, S. 1126 ff.; Schricker, S. 2026 ff.; Wandtke/Bullinger, S. 1440 ff.

557 Siehe dazu zum neuen Zollbestimmungen zum Schutz des geistigen Eigentums, Ganea, GRURInt. 2004, S. $532 \mathrm{f}$.
} 
sind nach $\int 3$ der chin. Zollbestimmungen die Waren, die das geistige Eigentum nach $\int 2$ der chin. Zollbestimmungen darstellen. Der Schutzrechtsinhaber hat nach \ 4 der chin. Zollbestimmungen einen Antrag auf den Zollschutz bei der Zollbehörde zu stellen.

b. Eintragung der Schutzrechte

Nach den neuen Vorschriften setzt der Zollschutz nicht mehr eine Eintragung des betreffenden Schutzrechts bei der Zollbehörde voraus. ${ }^{558}$ Die Gültigkeit der nunmehr freiwilligen Eintragung wurde von sieben auf zehn Jahre angehoben und kann um weitere zehn Jahre verlängert werden. 559

c. Beschlagnahme

Nach \ 12 der chin. Zollbestimmungen kann der Rechtsinhaber die Beschlagnahme der rechtsverletzenden Ware bei der örtlichen Zollbehörde beantragen. Die örtlichen Zollbehörden können rechtsverletzende Ware nicht von Amts wegen beschlagnahmen, aber sie haben nach $\int 16 \mathrm{~S} .1$ chin. Zollbestimmungen den Rechtsinhaber bei Verdacht auf eine Rechtsverletzung unverzüglich $\mathrm{zu}$ benachrichtigen. Stellt dieser nach $\int 16$ S. 2 chin. Zollbestimmungen innerhalb von drei Tagen einen Antrag auf Zollmaßnahmen und hinterlegt eine Sicherheit, so muss die Zollbehörde die betreffende Ware beschlagnahmen.

\section{d. Rechtsfolge}

Die beschlagnahmten Waren werden nach \ 27 chin. Zollbestimmungen von der Zollbehörde eingezogen, wenn die Rechtsverletzung durch die Untersuchung der Zollbehörde festgelegt wird. Stellt die Ein- oder Ausfuhr der rechtsverletzenden Ware eine Straftat dar, wird nach $\ 30$ chin. Zollbestimmungen die strafrechtliche Verantwortung nach dem chin. StGB verfolgt.

\section{E. Zusammenfassung}

Durch den vorliegenden Rechtsvergleich zwischen chinesischem und deutschem Recht zum Schutz des geistigen Eigentums zeigt sich große Ähnlichkeit in den zivilrechtlichen und strafrechtlichen Rechtsvorschriften bei den Rechtsverletzungen. Jedoch ist ein wesentlicher Unterschied zwischen den beiden Rechten zu ernennen, dass die zuständigen Verwaltungsbehörden auch eine Rolle bei den Rechtsverletzungen spielen und die Rechtsverletzungen durch die Verwaltungsakte verbieten. Das ist auch die Besonderheit des chinesischen international eher selten anzutreffenden zweispurigen Systems der Rechtsdurchsetzung.

\footnotetext{
558 Ganea, GRURInt. 2004, S. 532.

559 Ganea, GRURInt. 2004, S. 532.
} 


\section{Vierter Teil: Vertragrechtliche und kartellrechtliche Grundlagen zum Technologietransfer}

\section{A. Einführung in die Problematik}

Es handelt sich in diesem Teil um die vertraglichen und kartellrechtlichen Regelungen gegen Wettbewerbsbeschränkungen beim Technologietransfer auf den nationalen und internationalen Ebenen. Auf der einen Seite enthält das chin. VG Rechtsvorschriften, welche Anwendung auf die Vertragsgestaltung beim Technologietransfer finden und wettbewerbsverletzende oder wettbewerbsbeschränkende Vertragsklauseln verbieten. Auf der anderen Seite müssen Technologietransfer-Vereinbarungen im Anklang mit den kartellrechtlichen Regelungen stehen.

Nach dem Bericht von der WTO-Arbeitsgruppe über den Beitritt der VR China war das Land schon bereit, die Verabschiedung des chinesischen Kartellgesetzes in Gang zu bringen. ${ }^{560}$ Neuerdings ist das Antimonopolgesetz (Kartellgesetz) in der VR China vom Volkskongress am 30. August 2007 verabschiedet worden und wird erst am 1. August 2008 in Kraft treten. ${ }^{561}$

Früher waren die Regelungen Chinas gegen Wettbewerbsbeschränkungen nur vereinzelt in verschiedenen Gesetzen geregelt, wie z.B. das chinesische Preisgesetz ${ }^{562}$ und chinesische Gesetz gegen den unlauteren Wettbewerb. ${ }^{563}$ Das chinesische Gesetz gegen den unlauteren Wettbewerb ${ }^{564}$ war das grundlegende Gesetz zur Aufrechterhaltung der Wettbewerbsordnung auf dem Markt. Außerdem enthalten das Preisgesetz, das Gesetz über öffentliche Ausschreibung, das Strafgesetz und andere relevante Gesetze auch Bestimmungen gegen Wettbewerbsbeschränkungen und unlauteren Wettbewerb. ${ }^{565}$

Hier wird das EU-Kartellrecht im Vergleich mit dem neuen chinesischen Kartellgesetz berücksichtigt. Der EU-Vertrag enthält keine Sonderregelung im Hinblick auf den Technologietransfer. Einschlägig ist daher das allgemeine

\footnotetext{
${ }^{560}$ Vgl. Working Party Report on Accession of the People's Republic of China II. 8. Competition Policy 65. http://docsonline.wto.org/DDFDocuments/t/WT/ACC/CHN49.doc, WT/ ACC/ CHN/ 49

${ }^{561}$ Siehe Englische Fassung des neuen chinesischen Kartellgesetzes unter: http://www.fdi.gov.cn/pub/FDI_EN/Laws/law_en_info.jsp?docid=85714

Vgl. China's Anti-trust Regime by Emma Davies, Partner and Cheng Li Yow, Associate, Clifford Chance, Shanghai and London, China Law \& Practice, http://www. Chinalawandpractice.com Siehe auch im Internet unter: http://www.competitionlaw.cn/show.aspx?id=434\&cid=13

562 Verabschiedet und in Kraft getreten am 29. Dezember 1997

563 Verabschiedet am 2.9.1993 und in Kraft getreten am 1.12.1993

564 Siehe dazu, Wang, Xiaoye, Das Wettbewerbsrecht in China, Heidelberger Kommentar zum Wettbewerbsrecht, S. $854 \mathrm{ff}$.

565 Vgl. Working Party Report on Accession of the People's Republic of China II. 8. Competition Policy 65. http://docsonline.wto.org/DDFDocuments/t/WT/ACC/CHN49.doc, WT/ ACC/ CHN/ 49
} 
Kartellverbot in Art. 81 EGV, das der Sicherung eines Systems des unverfälschten Wettbewerbs im Gemeinsamen Markt dient. ${ }^{566}$ Außerdem besteht die von EU erlassene GVO-T'T, die auch in die Untersuchung hineingezogen wird.

Um die Wettbewerbsbeschränkungen zu bekämpfen und den zwischenstaatlichen Technologietransfer zu erleichtern, hat sich die internationale Gesellschaft sehr viel Mühe gegeben und versucht wettbewerbsrechtliche Regelungen in Bezug auf den internationalen Technologietransfer durch internationale Abkommen $\mathrm{zu}$ bestimmen. $\mathrm{Zu}$ erwähnen sind der Verhaltenskodex von UNCTAD und der Entwurf des IAC.

\section{Der Verhaltenskodex von UNCTAD 567}

\section{Grundzüge}

Im Rahmen der UNCTAD hat eine Diplomatische Konferenz stattgefunden, die „United Nations Conference on an International Code of Conduct on the Transfer of Technology“"568, welche die Aufgabe hatte, einen internationalen Verhaltenskodex für den Technologietransfer $\mathrm{zu}$ verabschieden. Der Verhaltenskodex hat eine in sich abgeschlossene Rechtsordnung für den internationalen Technologietransfer zum Inhalt, die sich nicht nur an Staaten, sondern auch an die einzelnen Wirtschaftssubjekte wendet und vom Wettbewerbsrecht über Kollisionsregeln bis hin $\mathrm{zu}$ Ausprägungen der Staatenkooperation eine Fülle von rechtlichen Aspekten regelt. ${ }^{569}$

Bislang haben die diplomatischen Verhandlungen und Konsultationen wegen der tief greifenden Meinungsunterschiede zwischen den beteiligten Staatengruppen noch zu keinem Ergebnis geführt und damit ist der Versuch ein internationales Einheitsrecht für den Technologietransfer zu schaffen gescheitert. Verhandelt wurde bei dieser Konferenz im wesentlichen zwischen drei Staatengruppen, der zahlenmäßig stärksten sog. Gruppe der 77, welche die Entwicklungsländer umfasst, der B-Gruppe, worunter die westlichen Industriestaaten verstanden werden, und der D-Gruppe der sozialistischen Länder mit Ausnahme von China, das keiner der drei „regionalen“ Gruppen angehört. ${ }^{570}$

Der Anwendungsbereich des Verhaltenskodex bezieht sich allein auf internationale Technologietransfer-Transaktionen. Die Bestimmungen über beschränkende Geschäftspraktiken im Kapitel 4 gelten allgemein als zentraler Teil des gesamten Verhaltenskodex.

2. Die Wettbewerbsregelungen des Verhaltenskodex ${ }^{571}$

In Sektion B des 4. Kapitels des Verhaltenskodex wird eine Liste verschiedener beschränkender Praktiken aufgeführt, die den nationalen, regionalen oder

\footnotetext{
${ }^{566}$ Kortunay, Ayhan, Patentlizenz- und Know-how-Verträge im deutschen und europäischen Kartellrecht, S. 140.

567 Siehe oben Erster Teil, A, Fn. 19.

568 Siehe dazu zur Entwicklung der „United Nations Conference on an International Code of Conduct on the Transfer of Technology“, Stoll, S. 90 ff.

569 Stoll. S. 89.

${ }^{570}$ Vgl. Fikentscher/Kunz-Hallstein, GRURInt. 1979, S. 437 f.; Timberg, IIC 1981, S. 133 ff.

571 Siehe dazu: Stoll, S. 120 ff.
} 
internationalen Regelungen vergleichbaren Inhalts wie

z. B. in GVO-TT, TRIPS entsprechen. Hier werden die einzelnen Praktiken wie folgt aufgestellt:

a. Rückgewährklauseln

b. Nichtangriffsklauseln

c. Wettbewerbsverbot

d. Beschränkungen von Forschung und Entwicklung

e. Beschränkungen hinsichtlich des Personals

f. Preisbindungen

g. Beschränkungen im Hinblick auf Veränderungen an der übertragenen Technologie

h. Ausschließliche Verkaufs- oder Vertretungsvereinbarungen

i. Unzulässige Bindungen und Beschränkungen des Technologienehmers

(Koppelungsgeschäfte)

j. Exportbeschränkungen

k. Patent-Pools, Cross-Licensing und ähnliche Vereinbarungen

1. Beschränkungen in der Werbung

m. Verpflichtungen nach Ablauf der Laufzeit der Verfügungsrechte

n. Beschränkungen nach Vertragsende

\section{Das internationale Kartellrechtsabkommen}

\section{Grundzüge}

Die „International Antitrust Code Working Group“572 hat am 10. Juli 1993 den Entwurf eines "International Antitrust Code" vorgelegt. ${ }^{573}$ Das Ziel war es, ein aus der Sicht der Wissenschaft begründetes Weltkartellrecht in Form eines Kartellrechtskodex zu erarbeiten, der sich als „Pluralateral Trade Agreement“ in die im Rahmen der Uruguay-Runde des GATT angestrebte WTO einfügt. ${ }^{574}$

2. Grundprinzipien des IAC

a. Die Regelungen des IAC beschränken den Anwendungsbereich des Abkommens auf grenzüberschreitende Vorgänge. Das Abkommen ist erst dann anwendbar, wenn sich die Wettbewerbsbeschränkung in mindestens zwei Vertragsstaaten auswirkt. ${ }^{575}$

b. Der IAC geht prinzipiell von den nationalen Kartellrechtsordnungen aus. Das Ziel ist nicht die Schaffung eines Einheitsrechts, sondern die Bewahrung der Besonderheiten der jeweiligen nationalen Kartellrechte als der Ausgangspunkt.

c. Der Grundsatz der Inländerbehandlung, wie er auch in art. 2 PVÜ, Art. 5 RBÜ und Art. III GATT niedergelegt ist. 576

\footnotetext{
${ }^{572}$ Die Arbeitsgruppe beruht auf privater Initiative. Siehe zur Zusammensetzung, Fikentscher, Draft International Antitrust Code, S. $71 \mathrm{f}$.

${ }^{573}$ Siehe den Text, Fikentscher, Draft International Antitrust Code, S. 53 ff.

574 Vgl. Fikentischer/ Drexl, RIW 1994, S. 93 ff.; Fikentscher, Draft International Antitrust Code, S. 35.

575 Fikentscher, Draft International Antitrust Code, S. 28.

${ }^{576}$ Vgl. Fikentscher, Draft International Antitrust Code, S. 25.; Nettesheim/Sander, S. 340.; Fikentscher, IIC 1996, S. 766.; Fikentscher, IIC 1995, S. 1002.
} 
d. Das Prinzip des Mindeststandards. Der IAC gewährleistet allen Vertragsstaaten einen gemeinsamen Mindestschutz des Wettbewerbs. Jedem Vertragsstaat steht jedoch frei, auf dieser Basis eine strengere Gesetzgebung zu erlassen.

e. Das Prinzip der internationalen Verfahrensinitiative. Art. 19 IAC verpflichtet die Vertragsstaaten zur Errichtung einer Internationalen Kartellbehörde (International Antitrust Authority, IAA). Während die PVÜ und die RBÜ in den Verbandsstaaten unmittelbar anwendbar sind, verweigern die meisten WTO-Vertragsstaaten den WTO-Regeln diese unmittelbare Wirkung. Deshalb verfügt die IAA über keine Kompetenz, unmittelbar gegen die Unternehmen in den Vertragsstaaten vorzugehen. Aber die IAA kann aufgrund eigener Initiative den IAC durch nationale Behörden und Richter durchsetzen.

3. Mindestanforderungen des IAC

Der IAC stellt Mindestanforderungen sowohl für das formelle nationale Kartellrecht als auch in Bezug auf die Ausgestaltung des materiellen Kartellrechts auf. Die Mitgliedstaaten haben die Pflicht zur Errichtung einer nationalen Kartellbehörde.

Der Schwerpunkt liegt auf der Ausgestaltung des materiellen Kartellrechts, soweit internationale Sachverhalte betroffen sind, Art. 3 Sec. 1 IAC. Er besteht aus den drei Pfeilen: Verbote im Bereich horizontaler und vertikaler Wettbewerbsbeschränkungen (Kapitel 2 IAC), Fusionskontrolle (Kapitel 3 IAC) und Verbot des Missbrauchs marktbeherrschender Stellungen (Kapitel 4 IAC). Hier wird aber nicht näher eingegangen.

\section{B. Die Rechtsbeziehungen im Vertrag über den}

\section{Technologietransfer}

Im Folgenden werden die Rechte und Pflichten jeder einzelner Vertragsparteien in den oben genannten vier unterschiedlichen Vertragstypen zum Technologietransfer ${ }^{577}$ untersucht.

\section{Vertrag zur Übertragung von Patentrechten und Rechten zur Patentanmeldung 578}

1. Rechte und Pflichten des Veräußerers von Patent und Patentanmeldungsrecht

a. Übertragung des Vertragsgegenstandes auf den Erwerber

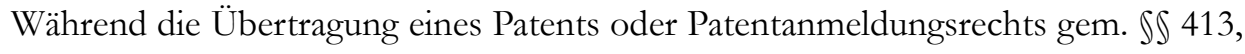
398 BGB durch Vertrag erfolgt, der formlos abgeschlossen werden kann ${ }^{579}$, kann der Vertrag über den Technologietransfer nach \342 Abs. 2 des chin. VG nur in

\footnotetext{
577 Siehe oben, Erster Teil, D, E, F.

${ }^{578}$ Siehe oben, Erster Teil, D.

579 Vgl. Benkard § 15 Rn. 5. S. 561.; Pagenberg/Geissler, Rn. 9. S. 486.; Ann/Barona, Rn. 165. S. 44.
} 
schriftlicher Form abgeschlossen werden. Zur rechtsgeschäftlichen Übertragung eines Gemeinschaftspatents und einer europäischen Patentanmeldung bedarf es allerdings der Schriftform des Übertragungsvertrages. ${ }^{580}$ Der Veräußerer ist nach \S 403, 413 BGB verpflichtet, eine öffentlich beglaubigte Urkunde über die Abtretung auszuhändigen. ${ }^{581}$

Außerdem ist der Veräußerer verpflichtet, dem Erwerber die technischen Unterlagen vollständig zu übergeben und seine volle Arbeitskraft zur Einweisung von Angestellten des Erwerbers zur Verfügung zu stellen.

b . Haftung

i. Rechtsmängelhaftung

Wendet man gem. \453 I BGB die kaufrechtliche Rechtsmängelhaftung auf den Schutzrechtskauf entsprechend an, so stehen dem Verkäufer im Falle eines Rechtsmangels die Befugnisse des $\int 437$ BGB zu. ${ }^{582}$ Nach der Entscheidung 583 von BGH übernimmt der Veräußerer eines Schutzrechts ohne Vereinbarung keine Haftung für dessen Rechtsbeständigkeit, da es sich bei einem Patentkauf um ein typisches Wagnisgeschäft handelt. ${ }^{584}$ Allerdings kann eine Verpflichtung zur Vertragsanpassung durch Kaufpreisherabsetzung aus den Grundsätzen über den Wegfall der Geschäftsgrundlage gem. \ 313 BGB erfolgen. Im Gegensatz dazu sieht \349 des chin. VG vor, dass der Technologiegeber die Haftung dafür hat, dass er der rechtmäßige Inhaber der Technologie ist.

ii. Sachmängelhaftung

Sachmangel ist eine Pflichtverletzung, die außer den Rechtsbehelfen des allgemeinen Leistungsstörungsrechts auch die kaufrechtliche Minderung auslöst. ${ }^{585}$ Da Verträge über die Patentvollveräußerungen ohne weiteres als Rechtskaufverträge zu qualifizieren sind, so ist die Sachmängelhaftung gem. \453 I BGB entsprechend anwendbar. Diese Haftung greift insbesondere ein, wenn die Erfindung nicht gem. \434 I 1 BGB die vereinbarte Beschaffenheit aufweist oder sich nicht für die nach dem Vertrag vorausgesetzte Verwendung eignet. ${ }^{586}$ Wegen

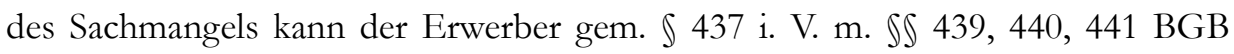
Nacherfüllung, Rücktritt, Minderung, Schadensersatz oder Aufwendungsersatz verlangen.

Im chinesischen Recht hat der Technologiegeber für die Vollständigkeit,

\footnotetext{
580 Vgl. Art. 39 Abs. 1, Art. 44 Abs. 1 GPÜ, Art. 72 EPÜ; EPA ABl. 1987, S. 216, 225.; Benkard, § 15 Rn. 6. S. 561.; Pagenberg/Geissler, Rn. 9. S. 486.

${ }^{581}$ Vgl. Pagenberg/Geissler, Rn. 12. S. 488.; Benkard, § 15 Rn. 7. S. 561.

${ }^{582}$ Vgl. Haedicke, GRUR 2004, S. 124

583 BGH GRUR 1982, S. 481 - Hartmetallkopfbohrer.

${ }^{584}$ Vgl. Pagenberg/Geissler, Rn. 21. S. 492.; Ann/Barona, Rn. 185. S. 47.; a. A. Haedicke, GRUR 2004, S. 124. „Richtiger dürfte es allerdings sein, die Rechtsmängelhaftung des Verkäufers eines Schutzrechts auch nach der Schuldrechtsreform als grundsätzlich verschuldensunabhängig anzusehen und das Vertretenmüssen i. S. von § 311a, 280, 276 BGB entsprechend zu interpretieren.“

585 Vgl. Ann/Barona, Rn. 189. S. 49.

${ }^{586}$ Haedicke, GRUR 2004, S. 124.
} 
Korrektheit, Nutzbarkeit und Geeignetheit für die nach dem Vertrag vereinbarte Verwendung nach \349 des chin. VG einzustehen. Liegt ein Sachmangel vor, dann haftet er nach $\iint 107$ ff. des chin. VG für die Vertragsverletzung.

c. Wettbewerbsverbot

Der Patentinhaber, der sein Patent veräußert, ist wie jeder Dritte während der Laufzeit des Patents an der Herstellung und dem Vertrieb von Erzeugnissen nach seiner Erfindung gehindert. ${ }^{587}$ Aber es kann ihm nicht verwehrt werden, in dem technischen Gebiet einer vom übertragenen Schutzrecht unabhängigen Verwertung technischer Ideen nachzugehen und auch andere nicht in den Schutzbereich des Vertragsschutzrechts fallende Erfindungen zu entwickeln. ${ }^{588}$

d. Nichtangriffsabrede

Den Veräußerer trifft die Nebenpflicht, das verkaufte Schutzrecht nicht mit Nichtigkeitsklage oder Löschungsantrag anzugreifen. 589 Bereits ohne Vereinbarung einer solchen Abrede sieht die Rechtsprechung die Einleitung einer Nichtigkeitsklage als unzulässige Rechtsausübung an, wenn der Kläger damit gegen Treu und Glauben verstößt. ${ }^{590}$

2. Rechte und Pflichten des Erwerbers von Patent und Patentanmeldungsrecht

a. Pflicht zur Zahlung des Kaufpreises

Die Hauptpflicht des Schutzrechtserwerbers besteht in der Zahlung des vereinbarten Entgelts. ${ }^{591}$ Nach \ 325 des chin. VG wird die Zahlungsweise des Preises bzw. Entgelts oder der Gebrauchsgebühr von den Vertragsparteien vereinbart. Sie können die Berechnung der Gesamtsumme auf einmal und ihre Zahlung auf einmal oder in Raten oder die Zahlung eines Anteils oder Zahlung eines Anteils zuzüglich eines Eintrittsgeldes wählen.

b. Kostentragung

Es ist zu empfehlen, die Kostentragungspflicht im Vertrag ausdrücklich zu vereinbaren. Beim Vertrag zur Übertragung eines Patentanmeldungsrechts trägt regelmäßig der Erwerber auch die Kosten für die Weiterführung des Prüfungsverfahrens. ${ }^{592}$

\section{Patentlizenzvertrag ${ }^{593}$}

\footnotetext{
587 Vgl. BGH GRUR 1951, S. 70, 71.; Benkard, § 15 Rn. 27. S. 565.

588 Vgl. BGH GRUR 1967, S. 378 - Schweißbolzen; GRUR 1966, S. 576 - Zimcofot; zum europäischen Recht EG-Kommission GRURInt. 1977, S. 130 - Reuter/BASF; GRUR 1976, S. 182 - AOIP/Beyrard; Pagenberg/Geissler, Rn. 27. S. 496.; Benkard, § 15 Rn. 27. S. 565 f.

${ }^{589}$ Vgl. BGH GRUR 1955, S. 535, 536 f.; GRUR 1989, S. 39, 40 - Flächenentlüftung; Ann/Barona, Rn. 177. S. 46.; Benkard, § 15 Rn. 27. S. 565.; Pagenberg/Geissler, Rn. 28. S. 496.

${ }^{590}$ Vgl. BGH Bl. 1965, S. 177, 178 - Vanal-Patent; GRUR 1956, S. 264 - Wendemanschette I; Pagenberg/Geissler, Rn. 28. S. 496.

591 Ann/Barona, Rn. 166. S. 45.

592 Vgl. Pagenberg/Geissler, Rn. 30. S. 496 f.

593 Siehe oben, Erster Teil, E.
} 
1. Rechte und Pflichten des Lizenzgebers

a. Nutzungseinräumung

Die Hauptpflicht des Lizenzgebers nach $\ 345$ des chin. VG besteht darin, dem Lizenznehmer ein positives Benutzungsrecht zu verschaffen. ${ }^{594}$ Bei der Umsetzung in die industrielle Fertigung hat der Lizenzgeber dem Lizenznehmer die erforderlichen Auskünfte zu erteilen und ihm die technischen Unterlagen und Unterweisungen zur Verfügung zu stellen. 595

b. Aufrechterhaltung und Verteidigung des Schutzrechts

i. Anmeldungs- und Aufrechterhaltungspflicht sowie Kostentragung

Da der Patentlizenzvertrag nur während der Schutzfrist des Patents nach $₫ 344$ des chin. VG gültig ist, ist der Lizenzgeber während der gesamten Vertragsdauer verpflichtet, die Vertragsschutzrechte aufrecht zu erhalten bzw. Schutzrechtsanmeldungen weiter zu verfolgen, soweit nicht der Lizenznehmer aufgrund vertraglicher Abmachungen selbst solche Erhaltungspflichten übernommen hat oder auch keine ausdrückliche Abrede besteht. ${ }^{596}$ Das gilt nicht nur bei einer einfachen Lizenz, sondern auch bei der Vereinbarung einer ausschließlichen Lizenz. Zur Erfüllung dieser Pflicht ist die Zahlung der Patentgebühren ebenso notwendig wie die ordnungsgemäße Durchführung im Zusammenhang mit der Aufrechterhaltung oder Weiterverfolgung der Schutzrechte anhängige Verfahren. ${ }^{597}$

ii. Unterlassungspflicht des Verzichts auf das Schutzrecht

Nach h. M. kann der Lizenzgeber ohne Zustimmung des Lizenznehmers auf das Schutzrecht nicht verzichten, wenn diesem eine ausschließliche Lizenz erteilt ist. ${ }^{598}$ Dagegen ist der ohne Zustimmung des einfachen Lizenznehmers erklärte Verzicht nach der herrschenden Meinung wirksam. ${ }^{599}$ Unabhängig von der Befugnis des Lizenzgebers, auf das Schutzrecht zu verzichten, macht sich der Lizenzgeber schadensersatzpflichtig im Falle seiner Ausübung, da er durch den Lizenzvertrag zur Aufrechterhaltung des Schutzrechts verpflichtet ist. 600

\footnotetext{
${ }^{594}$ Vgl. Benkard, § 15. Rn. 152. S. 594.; Stumpf/Groß, Rn. 243. S. 145.; Gitter, S. 399.; Henn, S. 186.; Gaul/Bartenbach, Rn. 371. S. K 136.; Krasser/Schmid, GRURInt. 1982 S. 328.; Osterrieth, Patentrecht, Rn. 347. S. 188.

${ }^{595}$ Vgl. Benkard, § 15 Rn. 152. S. 594.; Stumpf/Groß, Rn. 243. S. 145.; Gitter, S. 400.; Pagenberg/Geissler, Rn. 153 f. S. 112.; Rasch, S. 27.; Reimer, § 9 Rn. 46. S. 509;

596 Die herrschende Meinung geht davon aus, dass die Kosten für die Aufrechterhaltung des Schutzrechts bei einer ausschließlichen Lizenz, falls keine vertragliche Regelung besteht, der Lizenznehmer zu tragen hat. Vgl. Reimer, § 9 Rn. 59. S. 529.; Rasch, S. 53 f.; Lüdecke/Fischer, Rn. C 85. S. 258 ff.; Klauer/Möhring, § 9 Rn. 79. S. 500.; Busse, Patentgesetz § 15 Rn. 108. S. 414.; Pagenberg/Geissler, Muster 1 § 22 S. 50.

a. A. Henn, Rn. 327. S. 202 f.; Gaul/Bartenbach, Rn. 372. S. K 137.; Krasser/Schmid, GRURInt. 1982 S. 330 f.; Stumpf/Groß, Rn. 266. S. 152 f.; Osterrieth, Patentrecht, Rn. 348. S. 189.; Benkard, § 15 Rn. 152. S. 594.; Gitter, S. 401.; Schade, S. 82.

597 Vgl. Gaul/Bartenbach, Rn. 373. S. K 137.

598 Vgl. Lüdecke/Fischer, Rn. C 93. S. 269 ff.; Reimer, § 9 Rn. 96. S. 560.; Krasser/Schmid, GRURInt. 1982 S. 330.; a. A. Benkard, § 15 Rn 151. S. 594.; Stumpf/Groß, Rn. 267. S. 153.; Pfaff/Osterrieth, Rn. 134. S. 190.; Osterrieth, Patentrecht, Rn. 348. S. 189.;

599 Vgl. Reimer, § 9 Rn. 96. S. 560.; Stumpf/Groß, Rn. 267. S. 153.; Lüdecke/Fischer, Rn. C 93. S. 271.

${ }^{600}$ Vgl. Stumpf/Groß, Rn. 267. S. 153.; Osterrieth, Patentrecht, Rn. 348. S. 189.; Krasser/Schmid, GRURInt. 1982,
} 
iii. Verteidigungspflicht des lizenzierten Schutzrechts

Zur Verteidigung des lizenzierten Schutzrechts ist zunächst zu unterscheiden, ob ein Dritter einen rechtlichen Angriff auf das Schutzrecht vornimmt, oder ob er es durch Verletzungshandlungen als irrelevant hinstellt. 601

Im ersten Fall trifft den Lizenzgeber grundsätzlich die Verpflichtung, das lizenzierte Schutzrecht gegenüber Angriffen Dritter zu verteidigen. ${ }^{602}$ Ohnehin kann eine Nichtigkeits- oder Löschungsklage, die gegen das lizenzierte Schutzrecht erhoben wird, nur an den Lizenzgeber als den Schutzrechtsinhaber gerichtet werden. ${ }^{603}$ Der ausschließliche Lizenznehmer hat Anspruch auf den Schutz des Lizenzgebers gegenüber Angriffen Dritter. ${ }^{604}$

Umstritten ist auch, ob der Lizenzgeber verpflichtet ist, das Schutzrecht gegenüber Verletzungen Dritter zu verfolgen. Die herrschende Meinung verneint unabhängig davon, ob es um eine ausschließliche oder um eine einfache Lizenz geht, die Pflicht des Lizenzgebers, gegen Schutzrechtsverletzungen einzuschreiten. ${ }^{605}$ Aber nach Benkard ${ }^{606}$ trifft den Lizenzgeber grundsätzlich die Verpflichtung nach $\ 242$ BGB beim Lizenzvertrag, der eine einfache Lizenz mit einer Meistbegünstigungsklausel für den Lizenznehmer zum Gegenstand hat, ${ }^{607}$ da der Inhaber einer einfachen Lizenz wegen seiner schuldrechtlichen Position nicht gegen Patentverletzer vorgehen kann. ${ }^{608}$ Eine Verpflichtung zur Erhebung der Verletzungsklage besteht im Verhältnis zum ausschließlichen Lizenznehmer nicht, da er selbst befugt ist, die Rechte aus dem Patent geltend zu machen. ${ }^{609}$

Obwohl der Lizenznehmer aus eigenem Recht zur Verteidigung des lizenzierten Schutzrechts im Rahmen des ihm eingeräumten Benutzungsrechts befugt ist, meint Henn ${ }^{610}$, es geht nicht um das „Verteidigen können“, das auch dem einfachen Lizenznehmer im Wege der Bevollmächtigung grundsätzlich möglich wäre, sondern um das „Verteidigen müssen“, das dem Lizenzgeber deshalb obliegt, weil er zur Gleichbehandlung aller Lizenznehmer verpflichtet ist.

Die Erfahrung zeigt, dass in internationalen Technologietransfer- Verträgen in aller Regel der Lizenzgeber Herr der Verletzungs- und auch der Nichtigkeitsverfahren bleibt, der Lizenznehmer jedoch zur Unterstützung des Lizenzgebers verpflichtet ist. ${ }^{611}$ Dabei ist dies häufig der Fall, dass Abmachungen

\footnotetext{
S. 330.; Schade, S. 82.; Troller, GRURAusl. 1952, S. 108, 115.

601 Vgl. Lüdecke/Fischer, Rn. C 108. S. 292.

${ }^{602}$ Vgl. Benkard, § 15 Rn. 152. S. 594.; Klauer/Möhring, § 9 Rn. 59. S.482.; Lüdecke/Fischer, Rn. C 108. S. 292 ff.

${ }^{603}$ Gaul/Bartenbach, Rn. 380. S. K 139.

${ }^{604}$ RGZ 54, S. 272, 274.;

${ }^{605}$ Vgl. Stumpf/Groß, Rn. 279. S. 156.; Gitter, S. 402.; Lüdecke/Fischer, Rn. C 108. S. 295.; Pagenberg/Geissler, Rn. 266 ff. S. 164.; Reimer, § 9 Rn. 64. S. 534 f.; Busse, Patentgesetz, § 15 Rn. 114. S. 416.; Gaul/Bartenbach, Rn. 380. S. K 139.; Klauer/Möhring, § 9 Rn. 59. S. 482.; Krasser/Schmid, GRURInt. 1982 S. 331. „Trotz der auch insoweit gegebenen Aktivlegitimation des Lizenzgebers ist eine Pflicht zur Verteidigung mit der h.M. zu verneinen, weil das dingliche Benutzungsrecht des ausschließlichen Lizenznehmers Drittschutz genießt und der Lizenznehmer daher selbst über ausreichende Verteidigungsmöglichkeit verfügt.“

${ }^{606}$ a. A. Benkard, Rn. 153. S. 594.; Vgl. Henn, Rn. 321. S. 198 f.; Pfaff/Osterrieth, Rn. 157 ff. S. 196.

${ }^{607}$ BGH GRUR 65, S. 591, 595 - Wellplatten.

${ }^{608}$ Gaul/Bartenbach, Rn. 383. S. K 141.

609 Vgl. Benkard, \& 15 Rn. 55, 153. S. 572, 595.

${ }^{610}$ Vgl. Henn, Rn. 321. S. 199.

611 Vgl. Pfaff/Osterrieth, Rn. 158. S. 196.
} 
über die Kostenverteilung getroffen werden.

c. Gewährleistungspflichten

Die Gewährleistungspflichten des Lizenzgebers sind in $\ 349$ des chin. VG als der Generalklausel zum Vertrag über den Technologietransfer geregelt.

i. Gewährleistung für industrielle Verwertbarkeit und wirtschaftliche Eigenschaften

Nach h. M. ${ }^{612}$ haftet der Lizenzgeber sowohl für die industrielle Verwertbarkeit auch technische Ausführbarkeit und technische Brauchbarkeit genannt - der Erfindung als auch für zugesicherte Eigenschaften. Da der Lizenzvertrag üblich als ein gewagtes Geschäft ${ }^{613}$ angesehen wird, hat der Lizenzgeber dem Lizenznehmer vorbehaltlich entsprechender Zusicherungen nicht für wirtschaftliche Eigenschaften der lizenzierten Erfindung einzustehen. ${ }^{614}$

ii. Gewährleistung für Sachmängel(Tauglichkeitsmängel)

Die Haftung des Lizenzgebers für technische Ausführbarkeit und technische Brauchbarkeit erfolgt nach den Grundsätzen der Sachmängelhaftung. Die technische Ausführbarkeit wird so definiert, dass die technische Konzeption der Erfindung unter Einsatz üblicher Herstellungseinrichtungen, auch wenn diese erst geschaffen werden müssen, mit zumutbaren Aufwendungen innerhalb eines angemessenen Zeitraums verwirklicht werden kann. 615 Unter technischer Brauchbarkeit ist zu verstehen, dass mit dem Vertragsprodukt der von den Vertragspartnern erstrebte technische Verwendungszweck erreicht werden kann. ${ }^{616}$ Die Abgrenzung zwischen technischer Ausführbarkeit und Brauchbarkeit ist dabei praktisch unerheblich. ${ }^{617}$

In Anbetracht der Unsicherheit, die hinsichtlich der Haftung des Lizenzgebers besteht, verlangt der Lizenznehmer häufig ausdrückliche Zusicherungen. ${ }^{618}$ Zusicherung ist das vertraglich vom Lizenzgeber gegebene und vom Lizenznehmer angenommene Versprechen, dass der Lizenzgegenstand eine bestimmte Eigenschaft aufweist; für sie wird man kauf- und werkvertragsrechtlich

${ }^{612}$ Benkard, § 15 Rn. 176. S. 599.; Stumpf/Groß, Rn. 292. S. 161.; Gitter, S. 424.;Henn, Problematik, S. 123 f.; Henn, Rn. 307. S. 189.; Gaul/Bartenbach, Rn. 421. S. K 149.; Haver/Mailänder, S. 59.; Busse, Patentgesetz, § 15 Rn. 109. S. 414.; Rasch, S. 22 ff.; Pagenberg/Geissler, Rn. 59. S. 66.; Tetzner, § 9 Rn. 17. S. 703.; Klauer/Möhring, § 9 Rn. 72 f. S. 494 ff.; einschränkend Lüdecke/Fischer, Rn. B 10. S. 118.

${ }^{613}$ RGZ 78, S. 363, 367.; BGH GRUR 1957, S. 595, 597 - Verwandlungstisch; GRUR 1960, S. 44, 46 Uhrengehäuse; GRUR 1961, S. 27 - Holzbauträger; GRUR 1961, S. 466, 468 - Gewinderollkopf; GRUR 1970, S. 547, 549 - Kleinfilter; BGHZ 83, S. 283, 289 - Hartmetallkopfbohrer; Gitter, S. 418.; Gaul/Bartenbach, Rn. 421. S. K 149.; Osterrieth, Patentrecht, Rn. 350. S. 190.; Krasser/Schmid, GRURInt. 1982 S. 328.; Benkard, § 15 Rn. 158. S. 595.; Busse, Patentgesetz, § 15 Rn. 111. S. 415.;

${ }^{614}$ BGH GRUR 1985, S. 338, 340 f. - Brillengläser; GRUR 1965, S. 298, 301 - Reaktions-Messgerät; BGH GRUR 1978, S. 166 f. - Banddüngerstreuer; Vgl. Henn, Rn. 308. S. 189 f.; Stumpf/Groß, Rn. 292. S. 161.; Gitter, S. 419.; Gaul/Bartenbach, Rn. 421. S. K 149.; Busse, Patentgesetz, § 15 Rn. 111. S. 415.; Pagenberg/Geissler, Rn. 67. S. 70.; Pfaff/Osterrieth, Rn. 151. S. 194.; Tetzner, § 9 Rn. 17. S. 703.; Lindenmaier/Weiss, § 9 Rn. 21. S. 339.; Klauer/Möhring, § 9 Rn. 72. S. 494.; Reimer, § 9 Rn. 42. S. 507.; Osterrieth, Patentrecht, Rn. 357. S. 192.; einschränkend Lüdecke/Fischer, Rn. B 5. S. 112.

${ }^{615}$ RG v. 12. 6. 1942, GRUR 1943, S. 35.; BGH v. 26. 11. 1954, GRUR 1955, S. 338.; Vgl. Henn, Rn. 310. S. 191 f.; Stumpf/Groß, Rn. 299, 300, 303, 304. S. 163 ff.; Klauer/Möhring, § 9 Rn. 73. S. 495.; Reimer, § 9 Rn. 37. S. 500.; Rasch, S. 23.

${ }^{616}$ BGH v. 28. 6. 1979, GRUR 1979, S. 768.

617 BGH v. 1. 12. 1964, GRUR 1965, S. 298.

${ }^{618}$ Stumpf/Groß, Rn. 307. S. 166.; 
Grundsätze heranziehen können, so dass es darauf nicht ankommt, ob es technisch möglich ist, dem Lizenzgegenstand die zugesicherte Eigenschaft zu verleihen. ${ }^{619}$ Gibt der Lizenzgeber über die technische Brauchbarkeit des Lizenzgegenstandes hinaus Zusicherungen, so hat er dafür im Rahmen der Schadenersatzhaftung einzustehen. ${ }^{620}$

Das neue Kaufrecht gibt allerdings die Haftung des Verkäufers für Zusicherungen auf und ersetzt den Begriff der Zusicherung durch den der Garantie. ${ }^{621}$ Die Ansprüche des Käufers gehen nunmehr im allgemeinen Leistungsstörungsrecht sowie in $₫ 437$ BGB n. F. auf. ${ }^{622}$ Wenn man die Anwendbarkeit des Miet-/Pachtrechts für den Lizenzvertrag bejaht, dann haftet der Lizenzgeber gemäß IS 536a i. V. m. 536 BGB n. F.623

Bislang hat die Rechtsprechung allgemeines Leistungsstörungsrecht angewandt. Nach dessen Neuregelung stehen dem Lizenznehmer bei Tauglichkeitsmängeln des Vertragsgegenstands grundsätzlich ein Schadensersatzanspruch und ein Rücktrittsrecht zu, das nach Vollziehung des Lizenzvertrages nur zu einem Kündigungsrecht werden kann. ${ }^{624}$ Dann kann der Lizenznehmer Schadensersatz gemäß $\ 280,281$ BGB verlangen und/oder den Vertrag nach $₫ 323$ BGB außerordentlich kündigen.

iii. Gewährleistung für Rechtsmängel

Die Frage nach der Lizenzgeberhaftung stellt sich nicht nur bei Tauglichkeitsmängeln der Erfindung, sondern auch bei Mängeln, die in der Rechtsposition des Lizenzgebers auftreten. Bei der Lizenzgeberhaftung für Rechtsmängel sind drei Fallgruppen zu unterscheiden: ${ }^{625}$

aa. Die Haftung für den gegenwärtigen Bestand des Schutzrechts und die Verfügungsbefugnis des Lizenzgebers;

bb. Die Haftung für den künftigen Bestand des Schutzrechts und seinen Umfang; cc. Die Haftung für entgegenstehende Rechte Dritter.

Nach der Schuldrechtsreform führt das neue Leistungsstörungsrecht nicht zu einer geänderten Behandlung der Rechtsmängelhaftung: Für Fälle, die früher nach dem gesetzlich nicht geregelten Institut des Wegfalls der Geschäftsgrundlage ${ }^{626}$ gelöst wurden, gilt nun $₫ 313$ BGB. ${ }^{627}$

Die Vernichtbarkeit des lizenzierten Schutzrechts ist grundsätzlich kein haftungsauslösender Rechtsmangel. ${ }^{628}$ Aber nach den Grundsätzen des Wegfalls

\footnotetext{
${ }^{619}$ Vgl. Busse, Patentgesetz, § 15 Rn. 112. S. 415.; Ann/Barona, Rn. 129. S. 34 f.

${ }^{620}$ Vgl. Benkard, § 15 Rn. 175. S. 599.; Henn, Rn. 309. S. 190 f.; Gitter, S. 424.; Stumpf/Groß, Rn. 308. S. 167 ff.; Gaul/Bartenbach, Rn. 436. S. K 152.; Osterrieth, Patentrecht, Rn. 357. S. 192.; Klauer/Möhring, § 9 Rn. 74. S.

496.; Tetzner, § 9 Rn. 17. S. 705.; Reimer, § 9 Rn. 36. S. 498.; Busse, Patentgesetz, § 15 Rn. 112. S. 415.;

Pfaff/Osterrieth, Rn. 152. S. 194.; Pagenberg/Geissler, Rn. 60. S. 66.; Rasch, S. 27.

${ }^{621}$ Nach neuem Recht Beschaffenheitsgarantie, § 443 Abs. 1 BGB n. F.; Siehe dazu, Staudinger, §§ 433-487, § 443 Rn. 3, 9, 12, 14 ff, 24 ff.; Palandt, § 443 S. 643 ff.; Ann/Barona, Rn. 130. S. 35.

${ }^{622}$ Vgl. Ann/Barona, Rn. 130. S. 35.; Staudinger, §§ 433-487, § 437 S. 218 ff.; Palandt, § 437 S. 631 ff.

${ }^{623}$ Bisher $\S \S 537,538$ BGB a. F.; Siehe dazu, Stumpf/Groß, Rn. 330. S. 176 f.; Staudinger, §§ 535-562d, § 536 Rn. 49, § 536a Rn. 17 ff.; Palandt, §§ 536, 536a S. 743 ff.

${ }^{624}$ Vgl. Ann/Barona, Rn. 152. S. 41.

625 Vgl. Ann/barona, Rn. 142. S. 38.; Henn, Rn. 316. S. 194.; Gaul/Bartenbach, Rn. 466 ff. S. K 161 ff.

${ }^{626}$ Vgl. Palandt, § 313 Rn. 6. 25 ff. S. 497, 499 ff.; MünchKomm/Bearb. § 313 Rn. 4 ff. S. 1792 ff.

627 Vgl. Ann/Barona, Rn. 146. S. 39 f.; Stumpf/Groß, Rn. 357. S. 188.

${ }^{628}$ Vgl. Ann/Barona, Rn. 143. S. 38.; Krasser/Schmid, GRURInt. 1982, S. 339.
} 
der Geschäftsgrundlage erfolgt eine Vertragsanpassung ${ }^{629}$, wenn das lizenzierte Schutzrecht zwar noch nicht entfällt, seine Vernichtbarkeit aber schon offenbar oder mindestens wahrscheinlich geworden ist und das Schutzrecht seine geschäftliche Wirkung nicht mehr äußert. ${ }^{630}$ Wird das Schutzrecht nur teilweise vernichtet, bleibt der Lizenznehmer gemäß \$S 326 I 1 HS. 2, 441 III BGB n. F. zu einer geminderten Gegenleistung verpflichtet. Außerdem steht dem Lizenznehmer noch ein Kündigungsrecht nach $\int 314$ Abs. 1 BGB n. F. zu, wenn ihm das Festhalten am Vertrag trotz teilweisem Fortbestand des Schutzrechts nicht mehr zumutbar ist. ${ }^{631}$

\section{Rechte und Pflichten des Lizenznehmers}

a. Pflicht zur Zahlung der Lizenzgebühr

Der Lizenznehmer hat in der Regel eine Vergütung zu entrichten. Rechtsgrund der Entgeltpflicht ist regelmäßig eine ausdrückliche Parteivereinbarung. Fehlt diese, gilt eine Gegenleistung analog \$S 612 I, 653 I, 689 BGB als stillschweigend vereinbart, wenn die Lizenzeinräumung nach den Umständen nur gegen eine Vergütung zu erwarten ist. ${ }^{632}$ Die Berechnung der Lizenzgebühr ${ }^{633}$ kann als Umsatzlizenz, Stücklizenz oder Pauschallizenz erfolgen. ${ }^{634}$ Im chinesischen Recht ist die Zahlungspflicht des Lizenznehmers in \346 des chin. VG geregelt worden.

b. Pflicht zur Rechnungslegung

Neben der Hauptpflicht zur Zahlung der Lizenzgebühr trifft den Lizenznehmer die Pflicht zur Rechnungslegung, deren Umfang sich aus \259 BGB ergibt. ${ }^{635}$ Durch die Rechnungslegung wird der Lizenzgeber in der Lage sein, selbst eine Berechnung der Lizenzgebühren vorzunehmen bzw. die in der Regel vom Lizenznehmer vorgenommene Berechnung inhaltlich $\mathrm{zu}$ überprüfen. Im chinesischen Recht ist die Pflicht zur Rechnungslegung in $\int 325$ Abs. 3 des chin. VG geregelt, nach dem die Vertragsparteien das Einsichtsrecht in die Geschäftsbücher frei vereinbaren können.

Neben dem Umfang der Rechnungslegung ist der Abrechnungszeitraum, die Frist zur Vorlage der Rechnungslegung sowie die Fälligkeit der Lizenzgebühr zu definieren. ${ }^{636}$ Üblicherweise wird ein Abrechnungszeitraum von drei Monaten zugrunde gelegt. ${ }^{637}$ Mangels der Vereinbarung eines bestimmten

\footnotetext{
${ }^{629}$ Siehe dazu zur Vertragsanpassung, Busse, Patentgesetz, § 15 Rn. 103. S. 412 f.; MünchKomm/Bearb. § 313 Rn. $31 \mathrm{ff}$. S. $1798 \mathrm{f}$.

${ }^{630}$ Vgl. Ann/Barona, Rn. 143. S. 38.

631 Vgl. Pfaff/Osterrieth, Rn. 193. S. 203.; Stumpf/Groß, Rn. 357. S. 188.; Ann/Barona, Rn. 143. S. 39.

${ }^{632}$ Vgl. Krasser/Schmid, GRURInt. 1982, S. 332.; Ann/Barona, S. 20.

633 Siehe dazu zur Berechnung der Lizenzgebühr, Stumpf/Groß, Rn. 98 ff. S. 83 ff.; Benkard, § 15 Rn. 123 ff. S.

587 ff.; Pagenberg/Geissler, Rn. 172 ff. S. 120 ff.; Osterrieth, Patentrecht, Rn. 364 ff. S. 196 ff.; Gaul/Bartenbach, Rn. 628 ff. S. K 204 ff.; Lüdecke/Fischer, Rn. F 17 ff. S. 530 ff.; Pfaff/Osterrieth, Rn. 100 ff. S. 183 f.

${ }^{634}$ Pagenberg/Geissler, Rn. 172. S. 120.

${ }^{635}$ RGZ 127, S. 243, 244.; RG GRUR 1937 S. 1003, 1006.; Benkard, § 15 Rn. 145. S. 593.; Osterrieth, Patentrecht, Rn. 370. S. 198.; Gaul/Bartenbach, Rn. 711. S. K 243.; Pagenberg/Geissler, Rn. 220. S. 144.; Pfaff/Osterrieth, Rn. 100. S. 183.; Lüdecke/Fischer, Rn. F 39 ff. S. 552 ff.; Reimer, § 9 Rn. 56. S. 520.; Lindenmaier/Weiss, § 9 Rn. 52. S. 368.

${ }_{636}$ Pfaff/Osterrieth, Rn. 100. S. 183.; Vgl. Osterrieth, Patentrecht, Rn. 370. S. 198.

${ }^{637}$ Vgl. Pfaff/Osterrieth, Rn. 100.; Vgl. Osterrieth, Patentrecht, Rn. 371. S. 199.
} 
Abrechnungszeitraums ist innerhalb angemessener Frist abzurechnen. ${ }^{638}$

\section{c. Ausübungspflicht (Best-Efforts-Klausel)}

Durch die Vereinbarung einer Ausübungspflicht kann der Lizenzgeber verhindern, dass Unternehmen Patente als Defensivrechte erwerben, um die Verwertung der geschützten Technologie zu blockieren. ${ }^{639}$ Der Lizenzvertrag kann eine Verpflichtung des Lizenznehmers zur Ausübung der Lizenz ausdrücklich vorsehen.

Nach einhelliger Auffassung wird vielfach angenommen, dass sich eine Pflicht zur Ausnutzung des Patents bereits aus dem ausschließlichen Charakter des Lizenzvertrages ergebe. ${ }^{640}$ Sofern keine gegenteiligen Anhaltspunkte vorliegen, bedeutet die Vereinbarung einer ausschließlichen Lizenz im Zweifel, dass der Lizenznehmer zur Ausübung verpflichtet ist. ${ }^{641}$ Allgemein lässt sich der Grundsatz aufstellen, dass um so eher eine Ausübungspflicht bejaht werden muss, je mehr Befugnisse der Lizenznehmer eingeräumt erhält. ${ }^{642}$

Bei der einfachen Lizenz gehen die Meinungen auseinander. Grundsätzlich ist eine stillschweigende Ausübungspflicht zu verneinen, ${ }^{643} \mathrm{da}$ dem Lizenznehmer keine Monopolstellung eingeräumt wird. Auch dann nicht, wenn eine Stück- oder Umsatzlizenz vereinbart ist. ${ }^{644}$ Vielmehr kann der Lizenzgeber selbst aufgrund des Lizenzinhalts Waren herstellen und vertreiben oder weitere Lizenzen vergeben. Dagegen wird die Ausübungspflicht auch für die einfache Lizenz, z. T. allerdings mit Einschränkungen, von Möhring ${ }^{645}$ und Reimer ${ }^{646}$ bejaht.

Häufig finden sich auch so genannte Best-Efforts-Klauseln, die festlegen, dass der Lizenznehmer sich in bester (zumutbarer) Weise bemühen muss, der Ausübungspflicht nachzukommen. ${ }^{647}$ Neben der ausdrücklichen Feststellung der Best-Efforts-Klauseln ist deren Umfang zu ermitteln. Dazu gehört in erster Linie der Zeitpunkt der Produktionsaufnahme, der vom Entwicklungsstadium der lizenzierten Technologie abhängt. ${ }^{648}$ Nach Auffassung der Rechtsprechung ${ }^{649}$

\footnotetext{
${ }^{638}$ Vgl. Stumpf/Groß, Rn. 138. S. 105.; Osterrieth, Patentrecht, Rn. 371. S. 199.

${ }^{639}$ Vgl. Tokyo High Court, 24 II C (1993), 391 - Liquid fuel component m. Anm. v. Heath; Pagenberg/Geissler, Rn. 166. S. 118.; Osterrieth, Patentrecht, Rn. 372. S. 199.; Pfaff/Osterrieth, Rn. 106. S. 184.; Gitter, S. 407.

${ }^{640}$ Vgl. Benkard, § 15 Rn. 134. S. 590.; Stumpf/Groß, Rn. 151. S. 111.; Gaul/Bartenbach, Rn. 731. S. K 249.; Osterrieth, Patentrecht, Rn. 372. S. 199.; Pfaff/Osterrieth, Rn. 108. S. 184.; Pagenberg/Geissler, Rn. 166. S. 118.; Krasser/Schmid, GRURInt. 1982, S. 333 f.; Isay, S. 347.; Schade, S. 31. Tetzner, § 9 Rn. 20. S. 707.; Henn, ; Rasch, S. 55.; Lüdecke, GRUR 1952, S. 211.; Busse, Patentgesetz, Rn. 129. S. 418 f.; Henn, Rn. 278. S. 176.; Reimer, § 9 Rn. 55. S. 517.; Lindenmaier/Weiss, § 9 Rn. 53. S. 370.; Lüdecke, GRUR 1952, S. 211 ff.; Gitter, S. 407.; Klauer/Möhring, § 9 Rn. 78. S. 499.

${ }^{641}$ BGH GRUR 2000, S. 138 - Knopflochnähmaschinen.

642 Vgl. Lüdecke/Fischer, Rn. E 9. S. 444 f.; Gaul/Bartenbach, Rn. 732. S. K 249.

643 Vgl. Stumpf/Groß, Rn. 152. S. 111.; Gaul/Bartenbach, Rn. 733. S. K 250.; Groß, GRUR 1951, S. 369.; Rasch, S. 39 f.; Schade, S. 34.;;; Benkard, § 15 Rn. 135. S. 590.; Bechert, S. 31; Henn, Rn. 278 f. S. 176 f.;

Krasser/Schmid, GRURInt. 1982, S. 334.; Pagenberg/Geissler, Rn. 48. S. 256.; Gitter, S. 406.; Lindenmaier/Weiss, § 9 Rn. 53. S. 370.; Ann/Barona, S. 75.

${ }_{644}$ Vgl. Benkard, § 15 Rn. 135. S. 590.; Stumpf/Groß, Rn. 153. S. 112.; Tetzner, § 9 Rn. 20. S. 708.; Groß, GRUR 1951, S. 369.; Rasch, S. 39.; Lüdecke/Fischer, Rn. E 11. S. 451 f.

a. A. Klauer/Möhring, § 9 Rn. 78. S. 499.; Lindenmaier/Weiss, § 9 Rn. 53. S. 370.; Schade, S. 43 f.;

Gaul/Bartenbach, Rn. 733. S. K 250.; RG v. 3. 10. 1936, GRUR 1937, S. 37, 38; KG v. 8. 5. 1935, GRUR 1935, S. 892, 893; BGH v. 17. 3. 1961, GRUR 1961, S. 470, 471 - Mitarbeiterurkunde.

${ }_{645}$ Klauer/Möhring, § 9 Rn. 78. S. 499.

646 Reimer, § 9 Rn. 55. S. 517.

${ }^{647}$ Osterrieth, Patentrecht, Rn. 373. S. 200.

${ }^{648}$ Osterrieth, Patentrecht, Rn. 372. S. 200.; Vgl. Pfaff/Osterrieth, Rn. 111. S. 185.; Henn, Rn. 282. S. 177 f.;
} 
unterliegt die Beurteilung des Umfangs der Ausübungspflicht in besonderem Maße dem Grundsatz von Treu und Glauben nach \ 242 BGB mit Rücksicht auf die Verkehrssitte. ${ }^{650}$

Hierbei führt nicht jedes wirtschaftliche Risiko hinsichtlich der Herstellung und des Absatzes der Vertragsprodukte zur Einschränkung der Ausübungspflicht, da derartige Risiken in erster Linie vom Lizenznehmer zu tragen sind. ${ }^{651}$ Die Ausübungspflicht entfällt stets dann, wenn die Grenze der Zumutbarkeit überschritten wird. ${ }^{652}$ Ein Fall liegt vor, wenn sich der Lizenzgegenstand als technisch nicht verwertbar erweist oder auch wenn wirtschaftliche Gründe den Lizenznehmer daran hindern, den Lizenzgegenstand herzustellen oder zu vertreiben. ${ }^{653}$

Ein Bestandteil der Ausübungspflicht kann die Pflicht zur Einhaltung vom Mindestqualitätsstandard sein. Es kommt nicht nur darauf an, dass der Lizenznehmer herstellt, sondern auch wie er herstellt. In der Praxis vereinbaren die Parteien häufig, dass der Lizenznehmer bei der Herstellung des Lizenzgegenstandes Mindestqualitätsvorschriften einzuhalten hat. 654 Qualitätssicherungsvereinbarungen können nicht nur die Qualitätskontrollrechte des Lizenzgebers, sondern auch dessen Buchprüfungsrechte bezüglich der Lizenzgebühren erheblich verstärken. ${ }^{655}$ Für den Fall, dass die Produkte mit einem Lizenzvermerk oder einer Marke des Lizenzgebers versehen sind, kommen auch Ansprüche aus Produzentenhaftung für den Lizenzgeber in Betracht. ${ }^{656}$

Die Werbepflicht lässt sich als Bestandteil der Ausübungspflicht eines Lizenzvertrages auffassen. ${ }^{657}$ Der Werbung kommt für die Vermarktung der Vertragsprodukte eine ausschlaggebende Bedeutung zu. Für eine sachdienlichen Absatz der Vertragsprodukte müssen nicht nur die technischen, sondern auch die kaufmännischen, insbesondere die vertrieblichen Voraussetzungen geschaffen werden.

Falls der Lizenznehmer seiner Ausübungspflicht nicht nachkommt, verletzt er hiermit eine vertragliche Hauptpflicht. ${ }^{658}$ Der Lizenzgeber kann deshalb nach erfolglos gesetzter Frist zur Nachholung der Leistung gemäß \\ 281 Abs. 1, 280 Abs. 1 BGB n. F. Schadensersatz statt der Leistung verlangen und gemäß \ 323 Abs. 1 BGB zurücktreten. ${ }^{659}$

Stumpf/Groß, Rn. 154. S. 113.

649 BGH GRUR 2000, S. 138 - Knopflochnähmaschinen; OLG Düsseldorf WuW/E 1988, S. 900 Stützwinkelpatent; RG, MuW XIV, S. 328, 329; 1938, S. 206, 208 - Beleuchtungsanlage; BGH v. 11. 10. 1977, GRUR 1978, S. 166 - Banddüngerstreuer.

${ }^{650}$ Vgl. Pfaff/Osterrieth, Rn. 112. S. 185.; Osterrieth, Patentrecht, Rn. 373. S. 200.; Henn, Rn. 283. S. 178.; Gaul/Bartenbach, Rn. 757. S. K 254.

${ }^{651}$ Pfaff/Osterrieth, Rn. 112. S. 185.; Vgl. Henn, Rn. 282. S. 177.; Stumpf/Groß, Rn. 155. S. 113.

652 Vgl. Stumpf/Groß, Rn. 155. S. 113 f.; Henn, Rn. 283. S. 178.; Gaul/Bartenbach, Rn. 757. S. K 254.;

Pfaff/Osterrieth, Rn. 112. S. 185.; Osterrieth, Patentrecht, Rn. 373. S. 200.

${ }_{653}$ BGH GRUR 1970, S. 40, 42 - Musikverleger.

${ }^{654}$ Vgl. Pfaff/Osterrieth, Rn. 115. S. 186.; Osterrieth, Patentrecht, Rn. 375. S. 201.

655 Vgl. Stumpf/Groß, Rn. 156. S. 114.

656 Osterrieth, Patentrecht, Rn. 375. S. 201.

${ }^{657}$ KG v. 3. 9. 1938, GRUR 1939 S. 66.; Vgl. Pfaff/Osterrieth, Rn. 124. S. 188.; Osterrieth, Rn. 374. S. 200.; Stumpf/Groß, Rn. 162. S. 115 f.; Henn, Rn. 284. S. 178.; Benkard, § 15 Rn. 147. S. 594.; Gaul/Bartenbach, Rn. 749. S. K 253.; Schade, S. 74.

${ }^{658}$ Pfaff/Osterrieth, Rn. 114. S. 186.

659 Pfaff/Osterrieth, Rn. 114. S. 186. 
Die Ausübungspflicht findet ihre Grenze an der Unzumutbarkeit der Ausübung der Lizenz. Der Wegfall der wirtschaftlichen Voraussetzungen für die Ausübungspflicht hat zur unmittelbaren Konsequenz, dass die Ausübungspflicht selbst entfällt. ${ }^{660}$ Es kommt nicht darauf an, ob der Lizenznehmer den Lizenzvertrag gekündigt hat.

\section{d. Bezugspflichten}

Bezugspflichtvereinbarungen, nach denen der Lizenznehmer Roh-, Hilfs- und Zuschlagsstoffe, Vorprodukte, Zwischenerzeugnisse, Apparaturen, Zubehör oder Ersatzteile ausschließlich bei dem Lizenzgeber oder einem Dritten beziehen muss, spielen vor allem in Lizenzverträgen, die Verfahrenspatente zum Gegenstand haben, eine wichtige Rolle. ${ }^{661}$

Die Motivation im Einzelfall kann unterschiedlich sein: Zum einen kann die Bezugsbindung dazu dienen, über den Weg der Belieferung mit Rohstoffen etc. die Qualität des Lizenzproduktes mit zu beeinflussen und de facto die Qualitätssicherung des Lizenzproduktes zu gewährleisten. ${ }^{662}$ Die Einräumung einer Lizenz, verbunden mit einer Bezugspflicht, kann aber auch für den Lizenzgeber das einzige Mittel sein, um in Vertragsgebiet im Geschäft zu bleiben. ${ }^{663}$ Häufig wird vereinbart, dass der Lizenznehmer nur so viele Produkte herstellen darf, als er dazugehörige Einzelteile oder Baugruppen vom Lizenzgeber bezieht und dass er eine bestimmte Mindestmenge abzunehmen hat. ${ }^{664}$ So verspricht sich der Lizenzgeber durch den Vertrieb dieser Produkte an seinen Lizenznehmer ein Nebengeschäft, aus dem im Einzelfall ein wesentlich höherer Gewinn als aus den Lizenzgebühren erzielt werden kann.

Im chinesischen Recht dürfen jedoch keine wettbewerbsbeschränkende Vereinbarungen wie Bezugspflicht nach $\int 29$ chin. TIEVB im Technologieimportvertrag enthalten werden.

\section{e. Übermittelung von Verbesserungserfindungen}

Grundsätzlich besteht keine Pflicht zur Fortentwicklung der Vertragsprodukte durch den Lizenznehmer, weil es an sich die Aufgabe des Lizenzgebers ist, den Lizenzgegenstand so zur Verfügung zu stellen, dass er technisch ausführbar ist. ${ }^{665}$ Hat der Lizenznehmer Verbesserungen des lizenzierten Vertragsgegenstandes entwickelt, so ist er ohne eine ausdrückliche Abrede auch nicht verpflichtet, diese dem Lizenzgeber mitzuteilen oder ihm gar zur Verfügung zu stellen. ${ }^{666}$

\section{f. Nichtangriffsabrede}

\footnotetext{
${ }^{660}$ Vgl. Gaul/Bartenbach, Rn. 759. S. K 255.

661 Vgl. Stumpf/Groß, Rn. 197. S. 130 f.; Henn, Rn. 289. S. 181.; Pfaff/Osterrieth, Rn. 303. S. 235.; Gaul/Bartenbach, Rn. 777. S. K 260.

${ }^{662}$ Vgl. Pfaff/Osterrieth, Rn. 303. S. 235.; Stumpf/Groß. Rn. 198. S. 131.; Henn, Rn. 289. S. 182.

663 Stumpf/Groß, Rn. 199. S. 131.; Vgl. Henn, Rn. 289. S. 182.

664 Stumpf/Groß, Rn, 200. S. 132.; Vgl. Henn, Rn. 289. S. 182.

665 Vgl. Benkard, § 15 Rn. 150. S. 594.; Stumpf/Groß, Rn. 167. S. 119.; Pfaff/Osterrieth, Rn. 169. S. 199.; Osterrieth, Patentrecht, Rn. 377. S. 202.; Henn, Rn. 292. S. 183.

${ }^{666}$ Vgl. Gaul/Bartenbach, Rn. 810. S. K 270.; Stumpf/Groß, Rn. 171. S. 120.; Pagenberg/Geissler, Rn. 241. S. 152.; Pfaff/Osterrieth, Rn. 169. S. 199.
} 
Nichtangriffsabrede sind Rechtsgeschäfte, die den Vertragspartner verpflichten, sich bestimmter Angriffe auf ein bestimmtes Rechtsgut zu enthalten. ${ }^{667}$ Da der Lizenznehmer nach h. M. ${ }^{668}$ grundsätzlich nicht gehindert ist, das lizenzierte Patent mit der Nichtigkeitsklage anzugreifen, bedarf die Begründung einer wirksamen Nichtigangriffsverpflichtung des Lizenznehmers einer ausdrücklichen Vereinbarung. ${ }^{669}$ Auch ohne ausdrückliche Vereinbarung konnte eine Nichtigkeitsklage gegen ein Patent nach deutschem Recht unzulässig sein, wenn zwischen den Parteien ein besonderes Treuverhältnis bestand, so dass der Angriff des Lizenznehmers auf das Schutzrecht einen Verstoß gegen Treu und Glauben nach \ 242 BGB bedeuten würde. ${ }^{670}$ Dies galt insbesondere, wenn der Lizenzvertrag gesellschaftsähnlichen Charakter hatte oder sonst die Ausgestaltung im Einzelnen ein persönliches Vertrauensverhältnis zwischen den Vertragspartnern erkennen ließ. ${ }^{671}$ Die Wirkung der Nichtangriffsabrede bestand darin, dass sie zur Abweisung einer Nichtigkeitsklage als unzulässig führte, wenn sie dem Kläger entgegengehalten wurde. ${ }^{672}$ Aber eine Nichtangriffsklausel ist seit der 7. GWB-Novelle unwirksam geworden. ${ }^{673}$

g. Nachvertragliche Pflichten

Grundsätzlich hat der Lizenznehmer die Verwertung des Schutzrechts mit dem Ablauf des Lizenzvertrages einzustellen. Eine weitere Benutzung würde eine Verletzung des Schutzrechtes gemäß \9 PatG darstellen. Aber nach Beendigung des Lizenzvertrages steht dem Lizenznehmer nach \242 BGB jedoch ein Auslaufrecht zu. ${ }^{674}$ Gegenstände, die während der Vertragszeit vertragsgemäß hergestellt wurden, dürfen noch veräußert und in den Verkehr gebracht werden. ${ }^{675}$

Mit dem Ablauf des Lizenzvertrages muss der Lizenznehmer grundsätzlich alle ihm zur Herstellung und Verwertung des lizenzierten Gegenstandes überlassenen technischen und betriebswirtschaftlichen Unterlagen herausgeben. ${ }^{676}$ Wurde ihm begleitendes Know-how zur Verfügung gestellt, hat er dies auch nach

667 Vgl. Peetz, S. 27.

668 BGH v. 2. 3. 1956, GRUR 1956, S. 264 f. - Wendemanschette; v. 29. 1. 1957, GRUR 1957, S. 482, 483 -

Chenillefäden; v. 30. 11. 1967, GRURInt. 1969, S. 31 - Gewindeschneidapparat; Benkard, § 15 Rn. 141. S. 592.; Stumpf/Groß, Rn. 214. S. 135 f.; Gaul/Bartenbach, Rn. 841. S. K 278.; Henn, Rn. 336. S. 207.; Pfaff/Osterrieth, Rn. 313. S. 237.; Krasser/Schmid, GRURInt. 1982, S. 333.; Tetzner, § 9 Rn. 28. S. 715.; Lindenmaier/Weiss, § 9 Rn. 56. S. $373 \mathrm{f}$.

${ }^{669}$ Vgl. Osterrieth, Patentrecht, Rn. 382. S. 205.; Stumpf/Groß, Rn. 214. S. 135 f.;

${ }^{670}$ BGH GRUR 1956, S. 264 - Wendemanschette I; GRUR 1965, S. 135 - Vanal-Patent; Pagenberg/Geissler, Rn. 273. S. 166.; Gaul/Bartenbach, Rn. 842 f. S. K 278 f.; Henn, Rn. 337. S. 208.; Busse, § 81 Rn. 68. S. 1097.; Gitter, S. 408.; Klauer/Möhring, § 9 Rn. 80. S. 501.; Lindenmaier/Weiss, § 9 Rn. 56. S. 374.

${ }^{671}$ Vgl. Gaul/Bartenbach, Rn. 843. S. K 279.; Gitter, S. 408.; Henn, Rn. 337. S. 207 f.; Pagenberg/Geissler, Rn. 274. S. 166.; Lüdecke/Fischer, Rn. C 20. S. 167.; Busse, § 81 Rn. 76. S. 1098.; Tetzner, § 9 Rn. 28. S. 715.; Lindenmaier/Weiss, § 9 Rn. 56. S. 374.

${ }^{672}$ BGH v. 20. 5. 1953, BGHZ 10, S. 22 ff.; BGH NJW 1965, S. 491.; BGH GRUR 1971, S. $243-$

Gewindeschneidevorrichtung; BGH NJW - RR 1987, S. 1466.; v. 4. 10. 1988, GRUR 1989, S. 39, 40 -

Flächenentlüftung.

${ }^{673}$ Vgl. § 2 Abs. 2 GWB, Art. 5 Abs. 1 lit. C) TT-GFVO; BGH GRUR 1989, S. 39, 41 - Flächenentlüftung; EuGH GRURInt. 1989, S. 56 f. - Nichtangriffsklausel; Benkard, § 15 Rn. 141. S. 592.

${ }^{674}$ Vgl. Benkard, § 15 Rn. 203. S. 606.; Henn, Rn. 355. S. 217 f.; Pfaff/Osterrieth, Rn. 216. S. 207.; Stumpf/Groß, Rn. 217. S. 137.; Busse, § 15 Rn. 102. S. 412.

${ }^{675}$ RG Bl. 1906, S. 166, 167 - Jagdgewehrschussteile; BGH GRUR 1959, S. 528, 530 - Autodachzelt; Hamburg ZIP 1988, S. 925, 926.; Gitter, S. 433.; Krasser/Schmid, GRURInt. 1982, S. 341.; Ann/Barona, Rn. 137. S. 37. ${ }^{676}$ Vgl. Benkard, § 15 Rn. 204. S. 606.; Gitter, S. 433.; Stumpf/Groß, Rn. 219. S. 137.; Henn, Rn. 357. S. 218.; Ann/Barona, Rn. 138. S. 37. 
Vertragsbeendigung geheim zu halten. ${ }^{677}$

\section{Know-how-Vertrag678}

\section{Rechte und Pflichten des Know-how-Gebers}

a. Die Verpflichtung zur Mitteilung oder Überlassung des Know-how Die wesentliche Vertragspflicht des Know-how-Gebers nach \347 des chin. VG besteht darin, das im Vertragswerk konkret bezeichnete Erfahrungswissen dem Vertragspartner zu überlassen und die gegebenenfalls hierzu erforderlichen Maßnahmen, wie die Bereitstellung eigener Arbeitnehmer oder die Unterrichtung der Arbeitnehmer seines Vertragspartners, zu erbringen. ${ }^{679}$ Deshalb hat der Know-how-Geber alles zu tun, um dem Know-how-Nehmer die Ausübung seines Rechtes zu ermöglichen.

Es ist zu empfehlen, genaue Vereinbarungen im Know-how-Vertrag zu treffen, denn es muss nur im Einzelfall beurteilt werden, ob Nebenverpflichtungen unselbständig sind, je nachdem, ob sie der Verwirklichung des hauptsächlichen Vertragszwecks dienen. Vor allem bei Vertragspartnern, die ihren Sitz in Entwicklungsländern haben, muss der Know-how-Geber oft noch weitergehende Pflichten übernehmen, wie z. B. die Unterrichtung von Angestellten des Know-how-Nehmers in seinem Betrieb, die Anlernung von Arbeitskräften des Know-how-Nehmers, die Entsendung von Ingenieuren, die den Aufbau und die Überwachung der Produktion beim Partner durchzuführen haben, die Lieferung einer bestimmten Anzahl von Erzeugnissen aus der eigenen Produktion etc. ${ }^{680}$

\section{b. Haftung}

Know-how-Verträge sind mehr noch als Verträge über Schutzrechte gewagte Geschäfte. ${ }^{681}$ Grundsätzlich lässt sich also festhalten, dass der Know-how-Geber verpflichtet ist, dem Know-how-Nehmer das versprochene Wissen in geeigneter Form zu offenbaren. ${ }^{682}$ Die Rechtsmängelhaftung gem. $\int \$ 536$ ff. BGB greift ein, wenn das Know-how von einem Geheimpatent eines Dritten erfasst wird oder in (teilweiser) Abhängigkeit zum Patentschutz eines Dritten steht. ${ }^{683}$ Nach Stumpf ${ }^{684}$ hat der Know-how-Geber in aller Regel für die technische Ausführbarkeit und Brauchbarkeit des Know-how-Gegenstandes einzustehen. Nach Gaul, Bartenbach ${ }^{655}$ : Ausgehend von der Zuordnung des Know-how-Vertrages zu dem Vertragstyp des Dienstvertrages hat der Know-how-Geber solche Haftung wie

677 Vgl. Ann/Barona, Rn. 138. S. 37.; Henn, Rn. 358. S. 218 f.; Gitter, S. 433.; Pfaff/Osterrieth, Rn. 223. S. 209.

${ }^{678}$ Siehe oben, Erster Teil, F.

${ }^{679}$ Gaul/Bartenbach, Rn. 41. S. Q 18.; Vgl. Gitter, S. 450.; Stumpf, S. 55.

680 Stumpf, Der Know-how-Vertrag, Rn. 169. S. 132.

${ }^{681}$ Vgl. RGZ 163, S. 1, 8 f.; BGH GRUR 1960, S. 44, 46 - Uhrgehäuse; GRUR 1979, S. 768 f. - Mineralwolle; Benkard, § 15 Rn. 243. S. 617.

${ }_{682}$ Gitter, S. 451.; Vgl. Stumpf, S. 55 f.

683 Vgl. Benkard, § 15 Rn. 244. S. 617.

${ }^{684}$ Vgl. Stumpf, Der Know-how-Vertrag, Rn. 173. S. 133.

${ }^{685}$ Vgl. Gaul/Bartenbach, Rn. 45. S. Q 20. 
beim Lizenzvertrag nicht zu übernehmen, falls dies nicht im Vertragstext ausdrücklich niedergelegt worden ist. Entsprechend der Auffassung von Stumpf hat der Know-how-Geber nach $\ 347$ des chin. VG für die technische Ausführbarkeit und Brauchbarkeit des Know-how-Gegenstandes einzustehen. Im Fall eines Rechtsmangels haftet der Know-how-Geber nach $₫ 353$ des chin. VG, es sei denn, dass die beiden Vertragsparteien anders vereinbaren.

c. Unterlassung der eigenen Benutzung und Weitergabe des Know-how

Hat der Know-how-Geber ein ausschließliches Know-how zum Vertragsgegenstand gemacht, so darf er es im Regelfall weder selbst weiter benutzen, noch es Dritten zugänglich machen. ${ }^{686}$ Bei einer einfachen Vergabe von Know-how ist der Know-how-Geber dagegen immer noch berechtigt, das Know-how entweder selbst zu benutzen oder an Dritte zu vergeben. Es würde aber gegen Treu und Glauben verstoßen, wenn er das Know-how willkürlich ohne Gegenleistung weitergeben würde (Gratis- oder Frei-Know-how). ${ }^{687}$

d. Mitteilungs- und Verbesserungspflichten

Es besteht grundsätzlich keine Pflicht des Know-how-Gebers, Verbesserungen dem Know-how-Nehmer mitzuteilen und ihm Rechte daran einzuräumen, es sei denn, dass der Know-how-Geber eine solche Verpflichtung im Vertrag übernommen hat. ${ }^{688}$ Sind Verbesserungen in diesem Fall ohne Entgelt dem Know-how-Nehmer zu überlassen, so kann sich aus \242 BGB ein Anspruch auf eine angemessene Vergütung ergeben. 689

2. Rechte und Pflichten des Know-how-Nehmers

a. Vergütungspflicht des Know-how-Nehmers

Der Offenbarungspflicht des Know-how-Gebers steht als Hauptpflicht des Know-how-Nehmers die Vergütungspflicht nach $₫ 348$ des chin. VG gegenüber. ${ }^{600}$ Im Regelfall richten sich Art und Umfang nach den vertraglichen Vereinbarungen. ${ }^{691}$ Die Zahlungspflicht kann in Form einer einmaligen Pauschalsumme oder durch fortlaufende Zahlungen erfüllt werden. ${ }^{692}$

Bei der Stückgebühr muss der Know-how-Nehmer für jeden mit dem Know-how hergestellten Gegenstand einen bestimmten Betrag oder einen festgelegten Prozentsatz vom Verkaufspreis zahlen. Dagegen ist bei der Umsatzgebühr der Umsatz die relevante Bezugsgröße. Beide Vergütungsarten haben für den Know-how-Geber den Vorteil, dass er von einem wachsenden

${ }^{686}$ Gitter, S. 451.; Vgl. Stumpf, Der Know-how-Vertrag, Rn. 176. S. 135.; Stumpf, S. 57.; Benkard, § 15 Rn. 246. S. 617.

687 Stumpf, Der Know-how-Vertrag, Rn. 177. S. 135 f.; Vgl. Gitter, S. 451.

688 Vgl. Gitter, S. 451.; Stumpf, Der Know-how-Vertrag, Rn. 179. S. 136.; Stumpf, S. 57.

${ }^{689}$ RGZ 112, S. 361.; Vgl. Gitter, S. 451 f.; Stumpf, Der Know-how-Vertrag, Rn. 179. S. 136 f.; Stumpf, S. 58.

690 Gitter, S. 452.

${ }^{691}$ Siehe dazu Bewertungsfaktoren zur Festlegung der Know-how-Gebühr, Stumpf, Der Know-how-Vertrag, Rn.

93, S. 86 ff.; Stumpf, S. 35 ff.

${ }^{692}$ Gaul/Bartenbach, Rn. 43. S. Q 19. 
Absatz der mit seinem Know-how hergestellten Produkte profitiert. ${ }^{693}$

Durch eine Mindestgebühr wird der Know-how-Nehmer verpflichtet, unabhängig vom Umsatz oder von der Zahl der hergestellten Gegenstände in einem festgesetzten Zeitraum einen bestimmten Betrag zu zahlen. ${ }^{694}$ Dabei wird sichergestellt, dass der Know-how-Geber unabhängig vom Absatz eine Entschädigung allein für die Hingabe seiner Kenntnisse und Fertigkeiten bekommt. ${ }^{695}$

\section{b. Geheimhaltungspflicht des Know-how-Nehmers}

Neben der vorerwähnten Vergütungspflicht besteht eine wesentliche Verpflichtung des Know-how-Nehmers nach \348 des chin. VG, wenn der Know-how-Vertrag auf ein geheimes Erfahrungswissen gerichtet ist, darin, dass er alles unterlässt, was Dritten dieses Erfahrungswissen zugänglich machen könnte. ${ }^{696}$ Nach \352 des chin. VG lässt der Know-how-Nehmer einen Schadensersatzanspruch wegen der Vertragsverletzung entstehen, wenn er einem Dritten das Know-how ohne die Einwilligung des Know-how-Gebers überlässt.

c. Ausübungspflicht

Die Ausübungspflicht des Know-how-Nehmers entspricht dem Interesse des Know-how-Gebers. Dabei kann der Know-how-Geber einerseits seine Einnahmen sichern (z. B. bei Stück- bzw. Umsatzgebühr), andererseits soll durch das Know-how dieser Markt für den Know-how-Geber erhalten bleiben. ${ }^{697}$

Es fragt sich, wann eine Ausübungspflicht den Know-how-Nehmer trifft. Für Lizenzverträge wird eine Ausübungspflicht in neuerer Zeit grundsätzlich angenommen, wenn eine ausschließliche Lizenz erteilt wird. ${ }^{698}$ Bei der einfachen Lizenz gehen die Meinungen auseinander. Nach h. M. ${ }^{69}$ spricht die Erteilung einer solchen Lizenz gegen eine Ausübungspflicht. Dagegen wird die Ausübungspflicht zum Teil mit Einschränkungen von Möhring und Reimer bejaht. ${ }^{700}$

d. Verpflichtungen des Know-how-Nehmers nach Beendigung des Vertrages

\footnotetext{
693 Gitter, S. 452.

${ }^{694}$ Vgl. Stumpf, Der Know-how-Vertrag, Rn. 103. S. 95.

695 Vgl. Stumpf, Der Know-how-Vertrag, Rn. 103. S. 95.; Gitter, S. 452.

${ }^{696}$ Vgl. Gaul/Bartenbach, Rn. 52. S. Q 24.; Gitter, S. 453.

${ }^{697}$ Vgl. Gitter, S. 453.; Stumpf, Der Know-how-Vertrag, Rn. 123. S. 105.

698 Vgl. Stumpf, Der Know-how-Vertrag, Rn. 124. S. 105.; Gitter, S. 454.; Lüdecke, GRUR 1952 S. 211; Schade, S. 31.; Rasch, S. 55.; Lüdecke/Fischer, Rn. E 9. S. 445.; Stumpf, S. 47.

699 Siehe dazu zur herrschenden Meinung, Stumpf, Der Know-how-Vertrag, Rn. 125. S. 106.; Stumpf, S. 47 f.; Gitter, S. 453.; Lüdecke/Fischer, Rn. E 9. S. 446.; Schade, S. 35.; Rasch, S. 39.; Bechert, S. 31.; Groß, GRUR 1951 S. 369. „Die grundsätzliche Anerkennung der Ausführungspflicht durch die Kommentatoren, insbesondere bei Stücklizenz, führt also zu nichts anderem, als dass die Instanzgerichte die Beweislast als umgekehrt behandeln: nicht der Lizenzgeber hat die Ausführungspflicht zu behaupten und zu beweisen sondern der Lizenznehmer ihren Ausschluss.“

${ }^{700}$ KG GRUR 1935 S. 893. „Mit der herrschenden Meinung wird man beim Fehlen entsprechender Vertragserklärungen eine Ausübungspflicht des Lizenznehmers nach dem Sinn und Zweck eines Lizenzvertrages grundsätzlich als stillschweigend vereinbart annehmen können, wenn das Lizenzentgelt in einer Abgabe von jedem hergestellten Stück besteht, und zwar gleichgültig, ob es sich um eine Alleinlizenz oder eine einfache Lizenz handelt.“ Die herrschende Meinung war bis dahin in der Rechtsprechung nicht vorhanden. RG GRUR 1937 S. 38.; Klauer/Möhring, § 9 Rn. 78. S. 499.; Reimer, § 9 Rn. 55. S. 516 ff.
} 
Nach Beendigung des Know-how-Vertrages hat der Know-how-Nehmer diejenigen Unterlagen herauszugeben, die ein Geheimnis enthalten. ${ }^{701}$ Zugleich ist er verpflichtet, zukünftig jede Nutzung des Erfahrungswissens zu unterlassen. Im chin. VG ist die Herausgabepflicht nicht ausdrücklich vorgeschrieben, aber diese Pflicht kann man allerdings vertraglich vereinbaren.

\section{Das neue chinesische Kartellgesetz im Vergleich mit dem EU-Kartellrecht}

Das neue chinesische Kartellgesetz entspricht der gesetzgeberischen Rechtspraxis von den meisten Ländern in diesem Bereich. Es deckt die „klassischen“ drei Säulen des Kartellrechts ab: Verbot wettbewerbsbeschränkender Horizontal- bzw. Vertikalvereinbarungen (Kapitel 2), Verbot des Missbrauchs einer marktbeherrschenden Stellung (Kapitel 3) und Zusammenschlusskontrolle (Kapitel 4). ${ }^{702}$ In dieser Untersuchung werden nur die erst zwei Säulen angesprochen, die im engen Zusammenhang mit den Technologietransfer-Vereinbarungen stehen.

\section{Zweck}

Das neue chinesische Kartellgesetz beruht auf dem Grundsatz einer Sozialmarktwirtschaft mit freiem Wettbewerb und bezweckt den Schutz der rechtmäßigen Interessen der Unternehmer und Verbraucher und der öffentlichen Interessen sowie die Steigerung der wirtschaftlichen Effizienz.

\section{Anwendungsbereich}

Der Anwendungsbereich ist in Art. 2 des Kartellgesetzes geregelt. Dieses Gesetz findet Anwendung auf monopolistisches Verhalten während der Wirtschaftstätigkeiten in der Volksrepublik China. Auch findet dieses Gesetz Anwendung auf monopolistisches Verhalten, das sich im Wettbewerb des chinesischen Innenmarkts auswirkt, auch wenn es außerhalb des Geltungsbereichs dieses Gesetzes veranlasst wird.

In Art. 3 des Kartellgesetzes tritt monopolistisches Verhalten in drei Formen in Erscheinung, nämlich in Form von wettbewerbsbeschränkenden Vereinbarungen, Missbrauch einer marktbeherrschenden Stellung und wettbewerbsbeschränkenden Zusammenschlüssen. ${ }^{703} \mathrm{Nach}$ der ergänzenden Definition in Art. 3 des Kartellgesetzes handelt es sich bei wettbewerbsbeschränkenden Vereinbarungen um Vereinbarungen, Entscheidungen und andere abgestimmte Verhaltensweisen zwischen Unternehmen, die den Wettbewerb beschränken oder beseitigen oder dazu geeignet sind.

\footnotetext{
${ }^{701}$ Stumpf, Der Know-how-Vertrag, Rn. 158. S. 124.; Stumpf, S. 53.

${ }^{702}$ Vgl. Lorenz, GRURInt. 2006, S. 468.

703 Vgl. Lorenz, GRURInt. 2006, S. 468.
} 


\section{Verbot wettbewerbsbeschränkender Vereinbarungen}

Das neue Kartellgesetz verbietet jede Koordinierung des Marktverhaltens selbständiger Unternehmen sowohl im Horizontalverhältnis nach Art. 13 als auch im Vertikalverhältnis nach Art. 14, sofern keine Freistellung nach Art. 15 besteht. Horizontalverhältnisse bestehen zwischen anbietenden oder nachfragenden Unternehmen derselben Wirtschaftsstufe untereinander (z. B. zwischen Hersteller und Hersteller oder Händler und Händler), während Vertikalverhältnisse zwischen anbietenden oder nachfragenden Unternehmen einerseits und der Marktgegenseite andererseits bestehen (z. B. zwischen Hersteller und Händler oder zwischen Händler und Endverbraucher).

\section{Horizontalvereinbarungen}

Nach Art. 13 des Kartellgesetzes sind Monopolvereinbarungen zwischen konkurrierenden Unternehmen ${ }^{704}$ untersagt. Außerdem enthält Art. 13 noch fünf Beispiele für wettbewerbsbeschränkende Horizontalvereinbarungen:

a. konzertierte Preisgestaltung

Absprachen, die die Festlegung, die Beibehaltung oder die Änderung von Preisen für Produkte zum Gegenstand haben, sind verboten. ${ }^{705}$ Derzeit unterliegen Preisabsprachen dem Verbot des Art. 14 Nr. 1 des Preisgesetzes ${ }^{706}$ sowie $\int 4$ der vorläufigen Bestimmungen zur Beendigung monopolistischer Preisgestaltungen ${ }^{707}$, z. B. Preisabsprachen von den Unternehmern durch die Manipulation der marktbestimmten Preise oder Störung der Herstellung durch Absprache oder Missbrauch der marktbeherrschenden Stellung ( $\$$ 5-9 der vorläufigen Bestimmungen). ${ }^{708}$ \ 12 der vorläufigen Bestimmungen verpflichtet die Regierung und ihre untergeordneten Abteilungen, nicht rechtswidrig die marktbestimmten Preise zu intervenieren, sondern das Selbstbestimmungsrecht der Preise der Unternehmer zu respektieren.

Was die Rechtsfolgen der Preisabsprachen angeht, weist \10 der vorläufigen Bestimmungen auf Art. 40 des Preisgesetzes hin, der Richtigstellungsanordnungen, Beschlagnahme der illegalen Einkommen und Geldbuße in Höhe von fünfmal den rechtswidrigen Gewinnen.

Dieses Beispiel stimmt mit Art. 81 I lit. a) EGV teilweise überein. Anders als in Art. $81 \mathrm{I}$ lit. a) EGV wird die Festsetzung der Ankaufspreise in Art. 13 Nr. 1 des Kartellgesetzes nicht ausdrücklich erwähnt. ${ }^{709}$ Stattdessen ist ohne weitere

\footnotetext{
${ }^{704}$ Der Unternehmensbegriff des chinesischen Kartellrechts ergibt sich aus Art. 4 Abs. 1 des Gesetzesentwurfs: Unternehmen im Sinne des Gesetzesentwurfs können natürliche und juristische Personen sowie andere Organisationen sein, die auf dem relevanten Markt Waren herstellen oder umschlagen oder Dienstleistungen erbringen.

705 Lorenz, GRURInt. 2006, S. 469.

706 Verkündet am 29. Dezember 1997, in Kraft getreten am 1. Mai 1998.

707 Erlass Nr. 3 der staatlichen Kommission für Entwicklung und Reform vom 18. Juni 2003, in Kraft getreten am 1. November 2003.

${ }^{708}$ Vgl. Davies/Yow, S. 1.

709 Vgl. Lorenz, GRURInt. 2006, S. 469.
} 
Differenzierung von der Festsetzung der Produktpreise die Rede. ${ }^{710}$ Außerdem fehlt noch die Regelung über sonstige Geschäftsbedingungen im chinesischen Gesetzesentwurf. Art. 81 I lit. a) EGV verbietet die Festsetzung sonstiger Geschäftsbedingungen (Verbot von Konditionenkartellen). ${ }^{711}$

b. Beschränkungen der Herstellungs- oder Absatzmenge eines bestimmten Produkts. Das zweite Beispiel greift einen Teilaspekt des Art. 81 Abs. 1 lit. b) EGV auf, und zwar die Einschränkung oder Kontrolle der Erzeugung oder des Absatzes. ${ }^{712}$

c. Aufteilung von Rohstoff- oder Absatzmärkten

d. Beschränkungen des Erwerbs neuer Technologien oder Einrichtungen bzw. der Entwicklung neuer Produkte oder neuer Technologien, die den Teilaspekten des Art. 81 Abs. 1 lit. b) EGV entsprechen.

e. Boykottvereinbarungen. Wie im deutschen Kartellrecht das Boykottverbot nach $\int 21$ I GWB dürfen Unternehmen und Vereinigungen von Unternehmen nicht ein anderes Unternehmen oder Vereinigungen von Unternehmen in der Absicht, bestimmte Unternehmen unbillig zu beeinträchtigen, zu Liefersperren oder Bezugssperren auffordern. ${ }^{713}$

\section{Vertikalvereinbarungen}

Vertikalvereinbarungen bilden den Regelungsgegenstand des Art. 14 des Kartellgesetzes. Nach dieser Vorschrift ist es Unternehmen bei Geschäftstransaktionen untersagt, den Wiederverkaufspreis für Produkte zu beschränken oder andere Konditionen zu vereinbaren, die den Wettbewerb wesentlich beschränken oder ausschließen. ${ }^{714}$

\section{Freistellung}

Art. 15 des Kartellgesetzes enthält eine Reihe von Ausnahmetatbeständen von den Verboten der Art. 13 und Art. 14 des Kartellgesetzes. Der Katalog umfasst sechs Ausnahmen, bei deren Eingreifen wettbewerbsbeschränkende Vereinbarungen von diesen Verbotstatbeständen ausgenommen sind. Die Ausnahmetatbestände offenbaren zugleich die wirtschaftspolitischen Zielsetzungen, deren Erreichung das Kartellrecht nicht erschweren soll. 715 Wettbewerbsbeschränkende Vereinbarungen, die einem der folgenden Ziele dienen, sind zulässig, wenn sie zugleich auch die weiteren Voraussetzungen des Art. 15 des Kartellgesetzes erfüllen:

\footnotetext{
${ }^{710}$ Lorenz, GRURInt. 2006, S. 469.

711 Lettl, S. 51.

712 Lorenz, GRURInt. 2006, S. 469.

${ }^{713}$ Vgl. Lettl, S. 290.

${ }^{714}$ Vgl. Lorenz, GRURInt. 2006, S. 470.

${ }^{715}$ Vgl. Lorenz, GRURInt. 2006, S. 471.
} 
a. Der technologische Fortschritt und die Entwicklung neuer Produkte. Dieser Ausnahmetatbestand ist vergleichbar mit dem Freistellungstatbestand der Förderung des technischen Fortschritts des Art. 81 Abs. 3 EGV;

b. Die Verbesserung der Produktqualität, die Kostensenkung, die Effizienzsteigerung oder die Vereinheitlichung von Normen und Produktspezifikationen;

c. Die Verbesserung der Produktionseffizienz und die Steigerung der Wettbewerbsfähigkeit der kleinen oder mittleren Unternehmen (sog. Mittelstandkartelle);

d. Die Energieeinsparung, der Umweltschutz, die Katastrophenhilfe oder andere öffentlichen Interessen;

e. Die Bekämpfung einer wirtschaftlichen Depression, die Abmilderung der Folgen eines abrupten Nachfrageeinbruchs oder eines erheblichen Produktionsüberschusses.

f. Die Sicherstellung der Interessen im Außenhandel und bei der wirtschaftlichen Zusammenarbeit;

Die zusätzlichen Voraussetzungen entsprechen weitgehend denen des Art. 81 Abs. 3 EGV: Die Vereinbarung darf nicht zu einer wesentlichen Ausschaltung des Wettbewerbs auf dem relevanten Markt führen und die Verbraucher sind angemessen an dem entstehenden Gewinn zu beteiligen.

\section{Verbot des Missbrauchs einer marktbeherrschenden Stellung}

Art. 17 Abs. 1 des Kartellgesetzes enthält das Verbot des Missbrauchs einer marktbeherrschenden Stellung und stellt die Missbrauchstatbestände auf.

1. Missbrauchstatbestände

Art. 17 Abs. 1 des Kartellgesetzes beinhaltet einen nicht abschließenden Katalog missbräuchlichen Verhaltens, das marktbeherrschenden Unternehmen untersagt ist. Dazu gehören:

a. Einkauf oder Verkauf von Produkten $\mathrm{zu}$ missbräuchlichen hohen oder niedrigen Preisen;

b. Verkauf von Produkten unter den Gestehungskosten ohne sachlichen Grund;

c. die Geschäftsverweigerung ohne sachlichen Grund;

d. Ausschließlichkeitsvereinbarungen, in denen sich der Geschäftspartner des marktbeherrschenden Unternehmens ohne sachlichen Grund dazu verpflichtet, nur in Geschäftsbeziehungen mit ihm oder den von ihm bezeichneten Unternehmen einzutreten;

e. Kopplungsgeschäfte sowie die Einbeziehung anderer unangemessener 
Geschäftsbedingungen auf Druck des Marktbeherrschers;

f. die Diskriminierung gleichartiger Handelspartner ohne sachlichen Grund.

2. Marktbeherrschende Stellung

a. Begriff

Art. 17 Abs. 2 des Kartellgesetzes enthält die Definition der marktbeherrschenden Stellung: Eine solche besteht, wenn ein oder mehrere Unternehmen gemeinschaftlich dazu in der Lage sind, den Preis, die Produktmenge oder andere Konditionen in Geschäftsbeziehungen auf dem relevanten Markt zu kontrollieren oder andere Unternehmen vom Marktzutritt abzuhalten oder sie dabei zu behindern. ${ }^{716}$

b. Kriterien

Eine beherrschende Stellung auf dem relevanten Markt ergibt sich im Allgemeinen aus einem nicht abschließenden Katalog von sechs Kriterien nach Art. 18 des Kartellgesetzes.

i. Der Marktanteil des betreffenden Unternehmens und seiner Wettbewerber

ii. Die Fähigkeit zur Kontrolle eines Absatz- oder Nachfragemarktes

iii. Der Finanzstatus und die technischen Ressourcen eines Unternehmens

iv. Das Verhältnis anderer Unternehmen zu dem betreffenden Unternehmen und ihre Abhängigkeit von demselben

v. Der Zugang anderer Unternehmen zum relevanten Markt

vi. sonstige wettbewerbsrelevante Kriterien

c. Vermutungstatbestände

Art. 19 des Kartellgesetzes enthält drei Marktanteilsschwellen, bei deren Überschreiten das Bestehen einer marktbeherrschenden Stellung vermutet wird.

$\mathrm{Ab}$ einem Marktanteil eines Unternehmens von 50\% besteht eine Marktbeherrschungsvermutung. Verfügen zwei Unternehmen über einen gemeinsamen Marktanteil von zwei Dritteln oder drei Unternehmen über einen gemeinsamen Marktanteil von drei Vierteln, so begründet dies eine Vermutung für eine marktbeherrschende Stellung, sofern jedes der betreffenden Unternehmen einen Marktanteil von einem Zehnteln überschreitet.

${ }^{716}$ Vgl. Lorenz, GRURInt. 2006, S. 472. 


\section{Gruppenfreistellungsverordnung für Technologie-}

\section{Transfer-Vereinbarungen ${ }^{717}$}

\section{Einleitung}

Die Verabschiedung der GVO-TT und der sie begleitenden Leitlinien schließt ein längeres Konsultationsverfahren zur Reform der Gruppenfreistellungsverordnung (EG) Nr. 240/96 vom 31. 1. $1996^{718}$ ab, dessen letzte Phase die Kommission durch Veröffentlichung eines Entwurfs im EU-Amtsblatt vom 1. 10. $2003^{719}$ eröffnet hatte. ${ }^{720}$ Früher hatte die EG-Kommission am 30. 11. 1988 die „Verordnung (EWG) Nr. 556/89 zur Anwendung von Art. 85 Abs. 3 des Vertrages auf Gruppen von Know-how-Vereinbarungen“ verabschiedet. ${ }^{721}$ Diese Know-how -Verordnung ist am 1. 4. 1989 in Kraft getreten und galt bis zur Ablösung durch die GVO-TT am 1. 4. 1996722.

\section{Anwendungsbereich}

In den Erwägungsgründen der GVO-TT wird schon klar erklärt, dass der Gegenstand einer Technologietransfer-Vereinbarung die Vergabe einer Lizenz für eine bestimmte Technologie ist, sofern eine solche Vereinbarung die wirtschaftliche Leistungsfähigkeit steigert und sich positiv auf den Wettbewerb auswirkt. Ferner gilt die GVO-TT nur für Vereinbarungen, in denen der Lizenzgeber dem Lizenznehmer erlaubt, die lizenzierte Technologie zur Produktion von Waren oder Dienstleistungen zu nutzen.

Unter Technologietransfer-Vereinbarung zu verstehen ist in Art. 1 der GVO-TT die Patentlizenzvereinbarung, die Know-how-Vereinbarung, die Softwarelizenz-Vereinbarung oder gemischte Formen von solchen Vereinbarungen sowie die Übertragung von Patent-, Know-how- oder Software-Rechten und einer Kombination dieser Rechte. Immer noch nicht anwendbar ist die GVO-TT auf reine Urheberrechts- und Markenlizenzvereinbarungen. ${ }^{723}$

Know-how im Sinne dieser Verordnung ist eine Gesamtheit nicht patentierter praktischer Kenntnisse, die durch Erfahrungen und Versuche gewonnen werden und die geheim, wesentlich, identifiziert sind. ${ }^{724}$ Know-how-Vereinbarungen ${ }^{725}$ fallen unter dieselbe kartellrechtliche Beurteilung wie Lizenzverträge über

\footnotetext{
717 Siehe oben Erster Teil, A, Fn. 20.

718 ABIEG 1996 Nr. L 31, S. 2 ff.; Siehe dazu zu den Besprechungen dieser Verordnung, Meyer, GRURInt. 1997, S. 498 ff.; Stoffmehl, S. 305 ff.

${ }^{719}$ ABlEU Nr. C 235 v. 1. 10. 2003, S. 10.

720 Vgl. Lübbig, GRUR 2004, S. 483.

${ }^{721}$ Gaul/Bartenbach, S. K 340.

722 Verordnung (EG) Nr. 240/96 der Kommission vom 31. 1. 1996 zur Anwendung von Art. 85 Abs. 3 des Vertrages auf Gruppen von Technologietransfer-Vereinbarungen; ABl. EG 1996 Nr. L 31/2

${ }^{723}$ Gleichwohl soll die GVO-TT auf Lizenzen für urheberrechtlich geschützte Werke analog anwendbar sein, Leitlinien, Rn. 51.

${ }^{724}$ Vgl. Art. 1 Abs. 1 i) GVO-TT

725 Siehe dazu zu den Know-how-Vereinbarungen, Skaupy, GRUR 1964, S. 539 ff.; Hesse, GRUR 1985, S. 661 ff.; Mailänder, GRURInt. 1987, S. 523 ff.
} 
geschützte Erfindungen, wenn sie ein Betriebsgeheimnis betreffen. ${ }^{726}$

\section{Freistellung}

Nach Art. 2 Abs. 1 GVO-TT wird Art. 81 Abs. 1 EGV gemäß Art. 81 Abs. 3 EGV für nicht anwendbar erklärt auf Technologietransfer -Vereinbarungen zwischen zwei Unternehmen, die die Produktion der Vertragsprodukte ermöglichen, wenn die folgenden Voraussetzungen vorliegen ${ }^{727}$ :

1. Es handelt sich um eine Technologietransfer-Vereinbarung gemäß Art. 1 Abs. 1

b) GVO-TT;

2. Diese Technologietransfer-Vereinbarung enthält nach Art. 2 Abs. 2 Satz 1 GVO-TT Wettbewerbsbeschränkungen, die unter Art. 81 Abs. 1 EGV fallen;

3. Die Marktanteilsschwellen nach Art. 3 GVO-TT werden nicht überschritten sowie

4. Die Technologietransfer-Vereinbarung enthält nach Art. 4 GVO-TT keine Kernbeschränkung.

\section{Marktanteilsschwellen}

Nach Art. 3 Abs. 1 GVO-TT werden Vereinbarungen zwischen konkurrierenden Unternehmen nur dann von der GVO-TT erfasst, wenn der gemeinsame Marktanteil der Parteien auf dem betroffenen relevanten Technologie- und Produktmarkt 20\% nicht überschreitet. Bei Vereinbarungen zwischen nicht konkurrierenden Unternehmen gilt nach Art. 3 Abs. 2 GVO-TT eine Marktanteilsschwelle von 30\%.

\section{Kernbeschränkungen}

Enthält eine Technologietransfer-Vereinbarung eine Kernbeschränkung nach Art. 4 GVO-TT, dann ist die Freistellung nach Art. 2 GVO-TT ausgeschlossen. Die Kernbeschränkungen enthalten traditionelle kartellrechtliche Verbote. Insbesondere sind Preisbindungen, Beschränkungen des Outputs und die Aufteilung von Kunden oder Märkten nicht freigestellt. Eine Vielzahl von Ausnahmen ist in Bezug auf die Aufteilung von Kunden oder Märkten in Art. 4 Abs. 1 c) GVO-TT aufgeführt.

Die GVO-TT trennt bei den Kernbeschränkungen nach Vereinbarungen zwischen Wettbewerbern und Nicht-Wettbewerbern. Für die Zulässigkeit wettbewerbsbeschränkender Abreden zwischen Wettbewerbern gelten strengere Regeln als für die zwischen Nicht-Wettbewerbern. ${ }^{728}$

\section{Preisbindungsverbot}

\footnotetext{
${ }^{726}$ Vgl. BGHZ 17, S. 41, 51 - Kokillenguss; BGH GRUR 1980, S. 750 f. - Pankreaplex II; GRUR 1984, S. 753 Heizkessel-Nachbau; Benkard, § 15 Rn. 232. S. 614.

${ }^{727}$ Vgl. Stumpf/Groß, Rn. 638. S. 405.

${ }^{728}$ Vgl. Schumacher/Schmid, GRUR 2006, S. 6.
} 
Die GVO-T'T verbietet nicht nur direkte Vereinbarungen zwischen den Wettbewerbern über Festpreise, sondern auch Vereinbarungen über Mindestpreise, Höchstpreise oder Preisempfehlungen. Bei Vereinbarungen zwischen Nicht-Wettbewerbern sind Höchstpreise und Preisempfehlungen allerdings erlaubt, soweit sie nicht dieselbe Wirkung auf die Lizenznehmer durch die Ausübung von Druck oder die Gewährung von Anreizen wie Fest- oder Mindestpreise entfalten.

\section{Beschränkung des Outputs}

Die Beschränkungen des Outputs zwischen Wettbewerbern sind verboten mit Ausnahmen, dass die Beschränkungen des Outputs in einseitigen Lizenzvereinbarungen nur zu Lasten des Lizenzgebers, nicht aber zu Lasten des Lizenznehmers verboten sind. Außerdem darf einem einer Lizenznehmer die Produktions- und Verkaufsmenge in wechselseitigen Vereinbarungen in Bezug auf die Vertragsprodukte beschränkt werden.

\section{Aufteilung von Kunden oder Märkten}

Wichtigste Kernbeschränkung ist das Verbot, Kunden oder Märkte untereinander aufzuteilen. Allerdings hat diese Regelung auch eine Vielzahl von Ausnahmen. Ausdrücklich zugelassen sind für Wettbewerber in Art. 4 Abs. 1 lit. c ii GVO-TTT ausschließliche Lizenzen ${ }^{729}$ in nicht wechselseitigen Vereinbarungen. Alleinige Lizenzen ${ }^{730}$ sind nach Art. 4 Abs. 1 lit. c iii GVO-TT freigestellt, unabhängig davon, ob es sich um wechselseitige oder nicht wechselseitige Vereinbarungen handelt.

Außerdem können sich der Lizenzgeber und der Lizenznehmer in einer nicht wechselseitigen Vereinbarung gem. Art. 4 Abs. 1 lit. c iv GVO-TT verpflichten, aktive und passive Verkäufe in das Exklusivgebiet oder an die Exklusivkundengruppe zu unterlassen. Freigestellt ist auch nach Art. 4 Abs. 1 lit. c v GVO-TT die Beschränkung des aktiven Verkaufs von einem Lizenznehmer in das Gebiet anderer Lizenznehmer oder an die einem anderen Lizenznehmer zugewiesene Kundengruppe in einer nicht wechselseitigen Vereinbarung, wenn der Lizenznehmer bei der Lizenzerteilung nicht in Konkurrenz zum Lizenzgeber stand.

\section{Nicht freigestellte Beschränkungen}

Die GVO-TT trennt zwischen Kernbeschränkungen und nicht freigestellten Klauseln. Vereinbaren die Parteien eine Kernbeschränkung, dann gilt die Freistellung nach Art. 2 GVO-TT für den ganzen Vertrag nicht mehr. Die Vereinbarung einer nicht freigestellten Klausel hat lediglich zur Folge, dass nur diese Klausel als nicht freigestellt gilt und sich an Art. 81 I und III EGV messen lassen muss.

1. Rücklizenzierungsverpflichtung

\footnotetext{
${ }^{729}$ Siehe oben Erster Teil, F, III 1.

730 Siehe oben Erster Teil, F, III 2.
} 
Nach Art. 5 Abs. 1 lit. a und b GVO-T'T gilt die Freistellung nicht für die Verpflichtungen des Lizenznehmers, dem Lizenzgeber oder einem Dritten eine Exklusivlizenz für seine eigenen abtrennbaren Verbesserungen an der lizenzierten Technologie zu erteilen oder solche Verbesserungen auf den Lizenzgeber oder einen Dritten zu übertragen.

Der Grund für das Verbot von Rücklizenzierungsklauseln ist einfach: wenn der Lizenznehmer dazu verpflichtet wird, die von ihm entwickelten Erfindungen automatisch zurückzulizenzieren, fehlt es an einem Anreiz für den Lizenznehmer, die Technologie fortzuentwickeln. ${ }^{731}$

2. Nichtangriffsverpflichtung

Die Verpflichtungen des Lizenznehmers, die ihm lizenzierte Schutzrechte nicht anzugreifen, sind nach Art. 5 Abs. 1 lit. c GVO-TT nicht freigestellt. Allerdings zum Schutz des Lizenzgebers sieht die GVO-T'T vor, dass der Lizenzgeber den Vertrag sofort kündigen darf, wenn der Lizenznehmer das lizenzierte Schutzrecht angreift. ${ }^{732}$

3. Wettbewerbsverbote nicht konkurrierender Unternehmen

Die unmittelbaren oder mittelbaren Verpflichtungen zwischen nicht konkurrierenden Unternehmen als Vertragsparteien, die die Möglichkeit des Lizenznehmers, seine eigene Technologie zu verwerten, oder die Möglichkeit der Vertragsparteien, Forschungs- und Entwickelungsarbeiten durchzuführen, beschränken, sind nach Art. 5 Abs. 2 GVO-T'T nicht freigestellt, es sei denn, diese Beschränkung ist unerlässlich, um die Preisgabe des lizenzierten Know-how an Dritte zu verhindern. ${ }^{733}$

\section{Entzug des Rechtsvorteils}

Nach Art. 6 GVO-TT können die Kommission und die Wettbewerbsbehörden der Mitgliedstaaten Einzelvereinbarungen, die nicht die Voraussetzungen des Art. 81 Abs. 3 EGV erfüllen, den Rechtsvorteil der GVO-TT entziehen. Die Befugnis der Wettbewerbsbehörden der Mitgliedstaaten, den Rechtsvorteil zu entziehen, beschränkt sich auf Fälle, in denen der relevant räumliche Markt nicht größer ist als das Staatsgebiet des jeweiligen Mitgliedstaats. ${ }^{734}$

\section{Nichtanwendbarkeit der GVO-TT}

Aufgrund von Art. 7 kann die Kommission parallele Netze gleichartiger Vereinbarungen im Wege einer Verordnung aus dem Anwendungsbereich der GVO-TT ausschließen, wenn diese mehr als 50\% eines relevanten Markts erfassen. ${ }^{735}$

\footnotetext{
${ }^{731}$ Schumacher/Schmid, GRUR 2006, S. 9.

732 Vgl. Stumpf/Groß, Rn. 795 f. S. 468.

${ }^{733}$ Vgl. Stumpf/Groß, Rn. 799. S. 469.

${ }^{734}$ Vgl. Stumpf/Groß, Rn. 801. S. 470.

735 Vgl. Stumpf/Groß, Rn. 808. S. 472.
} 


\section{Freistellung im Einzelfall}

Schließlich ist zu beachten, dass mit der Durchführungs-VO Nr. 1/2003736 ein beträchtlicher Bedeutungsverlust der Gruppenfreistellungs-Verordnungen verbunden ist. Durch diese Durchführungs-VO wird das frühere Freistellungsmonopol der Kommission bei der Anwendung von Art. 81 Abs. 3 EGV durch das System der Legalausnahme ersetzt. ${ }^{737}$ Die Verordnung verwischt die Grenzen zwischen Einzel- und Gruppenfreistellung. Was nicht gruppenfreigestellt ist, kann heute dennoch unmittelbar aufgrund der Legalausnahme nach Art. 81 Abs. 3 EGV freigestellt sein. ${ }^{738}$

\section{E. Perspektive}

Heutzutage sind die Technologietransfer- Vereinbarungen in VR China nur unzureichend durch das chinesische Vertragsgesetz und Außenhandelsgesetz sowie Verwaltungs- Bestimmungen für den Technologieimport- und Export geregelt. Wegen der technischen Überlegenheit der ausländischen Lizenzgeber besteht auch dringend der Bedarf in China an dem chinesischen Kartellgesetz, um die inländische Industrie vor wettbewerbsbeschränkenden Geschäftspraktiken zu schützen.

Das neu verabschiedete chinesische Kartellgesetz enthält auch die drei Pfeile der modernen Kartellgesetzgebung. Deshalb kann man durchaus von den Erfahrungen des EU-Kartellrechts und des IAC lernen. In Sachen Technologietransfer-Vereinbarungen kann der Gesetzgeber auch eine spezielle Verordnung wie GVO-T'T erlassen, die eine Rahmengesetzgebung für die Technologietransfer-Vereinbarungen schafft. Jede solche Vereinbarung in Bezug auf den Technologietransfer wird freigestellt mit der Ausnahme, dass die in dieser Verordnung aufgestellten wettbewerbsbeschränkenden Geschäftspraktiken betroffen sind. Zu den Geschäftspraktiken ist der Verhaltenskodex von UNCTAD sehr hilfreich.

\footnotetext{
${ }^{736}$ Verordnung (EG) Nr. 1/2003 des Rates vom 16. 12. 2002 zur Durchführung der in den Artikeln 81 und 82 des Vertrags niedergelegten Wettbewerbsregeln, ABl. EG 2003 Nr. L 1/1.

${ }^{737}$ Vgl. Drexl, GRURInt. 2004, S. 717.

738 Vgl. Drexl, GRURInt. 2004, S. 719.
} 


\section{Zusammenfassung}

Am Ende dieser Untersuchung zur Steuerung des Technologietransfers in China sollen nun wesentliche Schlussfolgerungen zusammengefasst werden. Bei der Untersuchung wurde der Technologietransfer aus fünf Perspektiven betrachtet, nämlich, der Interessenlage beim Technologietransfer, der Gesetzgebung zum Technologietransfer, dem Schutz des geistigen Eigentums und den Rechtsbeziehungen der Vertragsparteien sowie der Wettbewerbspolitik.

1. Die Interessenlage Chinas als Entwicklungsland beim Technologietransfer Seit der Einführung der Reform und Öffnungspolitik am Ende der 70er bis zur Einleitung der sozialistischen Marktwirtschaft in den 90er Jahren hat sich Chinas Wirtschaft immer bemüht, um sich allmählich in die Weltwirtschaft zu integrieren. Ende 2001 wurde China nach langen Verhandlungen offiziell von WTO als Mitglied aufgenommen. Um sich der WTO-Rechtsordnung anzupassen und Verpflichtungen zur WTO zu erfüllen, hat China bereits seine Gesetze und Bestimmungen entsprechend geändert. Laut Statistiken über ausländische direkte Investitionen hat China seit einigen Jahren die USA überholt und ist nun das Land geworden, in dem man das größte ausländische Kapital investiert. Viele ausländische Unternehmen haben die Präsenz auf verschiedener Art und Weise gegründet, oftmals durch Lizenzierung oder durch Gründung von Joint Venture mit einem Chinesischem Partner usw. Der Strömung ausländischer Investitionen folgt auch der Transfer der Technologie. Denn die Technologie wird üblich als der Kern der Wettbewerbsfähigkeit des Unternehmens angesehen, befinden sich viele Unternehmen deshalb in einem Dilemma, dass sie einerseits die Überlegenheit in diesem großen Absatzmarkt durch ihre eigenen modernen Technologien erzielen und andererseits ihre globalen Interessen mangels des Schutzes der Technologie beeinträchtigt werden könnten.

2. Die Gesetzgebung zum Technologietransfer

Aufgrund der wirtschaftlichen Bedeutung der Technologie und der Interessenkonflikte beim Technologietransfer zwischen Staaten, Unternehmen und Individuellen bedarf es der rechtlichen Rahmenbedingungen auf internationaler und nationaler Ebene. Die Entwicklungsländer wie China als Technologienehmer brauchen eine nationale Rechtsordnung über den Technologietransfer. Einerseits muss die Rechtssicherheit für Schutzrechte und Know-how aus den Industrieländern als Technologiegeber gewährleistet werden. Andererseits müssen Wettbewerbsbeschränkende Vereinbarungen und der Missbrauch der durch Technologie-Monopol verursachten marktbeherrschenden Stellung kartellrechtlich verhindert werden. Angesichts der vorliegenden 
Interessenkonflikte hat China bei der Gesetzgebung zum Technologietransfer besonders zu berücksichtigen, dass die gesetzlichen Regelungen den Anreiz zum Technologietransfer anbieten und auch den Schutz des geistigen Eigentums gewährleisten.

Nach jahrelangen Verhandlungen ist die VR China mit Wirkung vom 11. Dezember 2001 der WTO beigetreten. Die wesentlichen Anforderungen des WTO Rechts an die Rechtsordnung Chinas lauten: Verstetigung des Rechtsstaatsprinzip und dessen kontinuierliche Weiterentwicklung in der Zivilund Wirtschaftsordnung. Die Zielsetzung der Gesetzgebung Chinas ist die Rechtsordnung Chinas mit dem Welthandelsrecht in Einklang zu bringen. Die wichtigen Prinzipien wie z. B. das Nichtdiskriminierungsprinzip, das Prinzip offener Märkte und Transparenzprinzip des WTO-Rechts sind durch die Rechtsreform ins chinesische Recht zu verankern.

\section{Der Schutz des geistigen Eigentums}

Durch den vorliegenden Rechtsvergleich zwischen chinesischem und deutschem Recht zum Schutz des geistigen Eigentums zeigt sich große Ähnlichkeit in den zivilrechtlichen und strafrechtlichen Rechtsvorschriften bei den Rechtsverletzungen. In Sachen Patentrechtsschutz sind im deutschen Recht erlaubte Benutzungshandlungen nach \$11 PatG, \$12 GebrMG von den Patentrechtsverletzungen ausgeschlossen, während im chinesischen Recht die Legalausnahmen in Art. 63 chin. PatG vorgeschrieben sind. Bei den Patentrechtsverletzungen könnten Ansprüche wie z. B. Unterlassungsanspruch, Schadensersatzanspruch, etc. sowohl im deutschen Recht als auch im chinesischen Recht entstehen. Es gilt auch bei den Rechtsverletzungen anderer Schutzrechte im zivilrechtlichen Schutz.

Bei der Rechtsdurchsetzung zum Schutz des geistigen Eigentums kann in dringlichen Fällen der Unterlassungsanspruch im Wege der einstweiligen Verfügung \$S 935, 940 ZPO im deutschen Recht geltend gemacht werden, die eine vorläufige Maßnahme darstellt. Im chinesischen Recht ist die Institution der einstweiligen Verfügung in den $\iint 74,92$ bis 99 chin. ZPG geregelt, die die Beweissicherung, die Vermögenssicherung und die Vorwegvollstreckung enthält.

Im deutschen Recht können die Waren bei der Ein- und Ausfuhr durch die Zollbehörde auf Antrag des Rechtsinhabers beschlagnahmt werden, wenn die Verletzung der Schutzrechte offensichtlich ist. Im chinesischen Recht kann der Rechtsinhaber die Beschlagnahme der rechtsverletzenden Ware bei der örtlichen Zollbehörde nach $\ 12$ der chin. Zollbestimmungen beantragen.

Jedoch ist ein wesentlicher Unterschied zwischen den beiden Rechten zu ernennen, dass die zuständigen Verwaltungsbehörden auch eine Rolle bei den Rechtsverletzungen spielen und die Rechtsverletzungen durch die Verwaltungsakte verbieten. Das ist auch die Besonderheit des chinesischen international eher selten anzutreffenden zweispurigen Systems der Rechtsdurchsetzung.

4. Die Rechtsbeziehungen der Vertragsparteien 
Im deutschen Recht ist die entgeltliche Übertragung der genannten Rechte nach $\$ 453 BGB in der Regel ein Rechtskauf. Der Lizenzvertrag wird heute allgemein als ein Vertrag sui generis angesehen und begründet ein Rechtsverhältnis eigener Art, das nach seinem besonderen wirtschaftlichen Inhalt sehr mannigfaltig sein, auch rechtlich eine gemischte Natur aufweisen kann. Im Gegensatz zum deutschen Recht ist der Übertragungs- und Lizenzvertrag im chinesischen Recht als ein bestimmter Vertragstyp in $\iint 322$ ff., 342 ff. des chin. VG geregelt worden.

Was die Rechtsbeziehungen der Vertragsparteien beim Vertrag über den Technologietransfer angeht, finden einige Vorschriften zwingend Anwendung. \ 329 des chin. VG schreibt vor, dass der Technologievertrag unwirksam ist, wenn er entweder die rechtswidrige alleinige Technologiebeherrschung oder die Verhinderung des Fortschritts der Technologie oder die Verletzung der Schutzrechte eines Drittes bezweckt. Nach \342 des chin. VG bedarf der Vertrag über den Technologietransfer der schriftlichen Form. Nach \343 des chin. VG darf der Vertrag über den Technologietransfer den Wettbewerb und die Weiterentwicklung der Technologie nicht beschränken, wenn es zur Vereinbarung über den Umfang des übertragenen oder lizenzierten Patents oder Know-hows kommt. Die Rücklizenzierungsverpflichtung und Nichtangriffsverpflichtung werden nach $\int 30$ des chin. AHG als wettbewerbswidrig behandelt, wenn sie die freie Wettbewerbsordnung im Außenhandel gefährden. Die Rücklizenzierungsverpflichtung ist auch in \354 des chin. VG geregelt worden. Die Vertragsparteien können nach dem Gegenseitigkeitsprinzip die Nutzung der abtrennbaren Verbesserungen frei vereinbaren. Liegt keine Vereinbarung vor oder wenn die Vereinbarung durch die Auslegung nach \61 des chin. VG immer noch unklar bleibt, wird kein Nutzungsrecht an den Technologiegeber gewährt.

Außerdem sind die Hauptpflichten der Vertragsparteien vertragrechtlich vorgeschrieben, bzw. die Verpflichtung zur Übertragung des Vertragsgegenstandes nach SS 345, 347 des chin. VG, die Haftung des Technologiegebers für Rechtsmangel und Sachmangel nach $\iint 347,349,353$ des chin. VG sowie die Vergütungspflicht nach $\iint 346,348$ des chin. VG und die Geheimhaltungspflicht des Technologienehmers nach $\iint 348,350$ des chin. VG.

Die übrigen Rechtsvorschriften in diesem Kapitel sind meistens rechtsdispositiv. Es werden den Vertragsparteien viele Freiräume überlassen, Vereinbarungen zu treffen. In der Rechtspraxis ist es sehr empfehlenswert, dass Rechte und Pflichten der Vertragsparteien beim Technologietransfer möglichst genau und detailliert vereinbart werden, damit die Streitigkeiten bei der Durchsetzung des Technologietransfers vermieden werden können.

Ein Rechtsvorrang für Gesetzesvorschriften und Verwaltungsbestimmungen über Technologieimport- und -exportvertrag befindet sich in $\ 355$ des chin. VG.. Nach S 29 der chin. TIEVB sind insbesondere Kopplungsgeschäfte, Vergütungspflicht nach Ablauf des Patents, Rücklizenzierungsverpflichtung, Bezugspflicht und Preisabsprache verboten.

5. Die Wettbewerbspolitik 
Nach Art. 2 GVO-TT wird Art. 81 Abs. 1 EGV gem. Art. 81 Abs. 3 EGV für nicht anwendbar erklärt auf Technologietransfer-Vereinbarungen zwischen zwei Unternehmen, die die Produktion der Vertragsprodukte ermöglichen. Dann bleibt Art. 4 GVO-T'T trotzdem unberührt, wenn die Vereinbarungen eine Kernbeschränkung beinhalten. Im neuen chinesischen Kartellgesetz sind die Kernbeschränkungen in Art. 13, 14 des chin. KG aufgestellt. Sie werden dann durch Art. 15 des chin. KG freigestellt, wenn die Technologietransfer-Vereinbarungen einen der Ausnahmetatbestände erfüllen. Es würde dazu führen, dass der Technologiegeber willkürlich den Preis, zu dem der Technologienehmer seine Produkte an Dritte verkauft und das Output sowie den Kundenkreis beschränken könnte. Es würde dem Zweck dieser Gesetzgebung nicht entsprechen. Aber es bleibt in der zukünftigen Rechtspraxis zu erklären. 


\section{Antimonopolgesetz der VR China ${ }^{739}$}

Verabschiedet auf der 29. Sitzung des Ständigen Ausschusses des 10. Volkskongresses und verkündet am 30.8.2007

Inhalt:

1. Kapitel: Allgemeine Regeln

2. Kapitel: Monopolvereinbarungen

3. Kapitel: Mißbrauch einer marktbeherrschenden Stellung

4. Kapitel: Unternehmenszusammenschluß

5. Kapitel: Mißbrauch von Verwaltungsmacht, um Wettbewerb auszuschließen oder einzuschränken

6. Kapitel: Untersuchung von als monopolistisch verdächtigen Handlungen

7. Kapitel: Rechtliche Verantwortung

8. Kapitel: Ergänzende Regeln

1. Kapitel: Allgemeine Regeln

$\int 1 \mathrm{Um}$ monopolistischen Handlungen vorzubeugen und sie zu unterbinden, fairen Marktwettbewerb zu sichern, die Effizienz wirtschaftlicher Abläufe zu steigern, die Interessen der Verbraucher und das Allgemeininteresse $<2>\mathrm{zu}$ schützen und die gesunde Entwicklung der sozialistischen Marktwirtschaft zu fördern, wird dies Gesetz bestimmt.

$\int 2$ Dies Gesetz wird auf monopolistische Handlungen bei wirtschaftlichen Aktivitäten im Gebiet der VR China angewandt; es wird auf monopolistische Handlungen außerhalb des Gebiets der VR China angewandt, die sich Marktwettbewerb innerhalb des Gebiets ausschließend oder einschränkend auswirken.

\3 Monopolistische Handlungen nach diesem Gesetz schließen ein:

1. Von Unternehmen getroffene Monopolvereinbarungen;

2. Mißbrauch der marktbeherrschenden Stellung von Unternehmen;

3. Unternehmenszusammenschlüsse, die zum Ergebnis haben oder haben können, Wettbewerb auszuschließen oder einzuschränken.

$\int 4$ Der Staat bestimmt der sozialistischen Marktwirtschaft entsprechende

${ }^{739}$ Übersetzung, Anmerkungen, (C) an beiden: F.Münzel, Hamburg 
Wettbewerbsregeln und wendet sie an, vervollkommnet die makroökonomische Kontrolle und vollendet ein einheitliches, offenes, wettbewerbliches, geordnetes Marktwirtschaftssystem.

\5 Unternehmen können in fairem Wettbewerb und freiwilligen Verbindungen nach dem Recht Zusammenschlüsse durchführen, das Ausmaß ihres Betriebs erweitern, ihre Fähigkeit zu Marktwettbewerb erhöhen.

\6 Unternehmen, die eine marktbeherrschende Stellung innehaben, dürfen sie nicht mißbrauchen, um Wettbewerb auszuschließen oder einzuschränken.

$\int 7$ Der Staat schützt die legale Unternehmenstätigkeit der Unternehmen in Lebensadern der Volkswirtschaft oder die Sicherheit des Staates berührenden Branchen, in denen die staatseigene Wirtschaft eine kontrollierende Stellung hat, und in Branchen, die $<3>$ nach dem Recht etwas ausschließlich betreiben oder vertreiben, und überwacht, steuert und kontrolliert nach dem Recht das unternehmerische Handeln dieser Unternehmen und die Preise ihrer Waren und Dienstleistungen, die Interessen der Verbraucher schützend und den technischen Fortschritt fördernd.

Die Unternehmen der Branchen nach dem vorigen Absatz müssen nach dem Recht betrieben werden, Treu und Glauben wahren, strikte Selbstdisziplin üben, die Aufsicht der Allgemeinheit akzeptieren, sie dürfen ihre kontrollierende Stellung oder ihre Stellung als ausschließliche Betreiber oder Verkäufer nicht nutzen, um die Interessen der Verbraucher zu schädigen.

\8 Verwaltungsbehörden und von Gesetzen und anderen Rechtsnormen ermächtigte, Funktionen beim Management öffentlicher Angelegenheiten ausübende Organisationen dürfen Verwaltungsmacht nicht mißbrauchen, um Wettbewerb auszuschließen oder einzuschränken.

$\int 9$ Der vom Staatsrat errichteten Antimonopolkommission obliegt es, die Antimonopolarbeit zu organisieren, zu koordinieren und anzuleiten; sie übt die folgenden Amtsaufgaben aus:

1. Sie überlegt und entwirft einschlägige Wettbewerbsrichtlinien;

2. sie organisiert Untersuchungen und Bewertungen der Wettbewerbsverhältnisse auf dem Markt insgesamt und gibt Bewertungsberichte heraus;

3. sie setzt Antimonopolwegweiser fest und gibt sie heraus;

4. sie koordiniert die rechtsvollziehende Arbeit der Antimonopolverwaltung;

5. vom Staatsrat bestimmte andere Amtsaufgaben.

Zusammensetzung und Arbeitsregeln der Antimonopolkommission des Staatsrats werden vom Staatsrat bestimmt.

$\int 10$ Dem vom Staatsrat bestimmte Organ, das die Amtsaufgabe des Vollzugs des Antimonopolrechts übernimmt (im Folgenden: das 
Antimonopolrecht-Vollzugsorgan des Staatsrats) obliegt entsprechend den Vorschriften dieses Gesetzes der Vollzug des Antimonopolrechts.

Das Antimonopolrecht-Vollzugsorgan des Staatsrats kann, wenn es die Arbeit erfordert, entsprechende Organe der Volksregierungen der PAS ermächtigen, entsprechend den Vorschriften dieses Gesetzes Verantwortung für den Vollzug von Antimonopolrecht zu übernehmen.

$\int 11$ Branchenverbände müssen die Selbstdisziplin der Branche stärken, die Unternehmen ihrer Branche zu Wettbewerb nach dem Recht anleiten und die Ordnung des Marktwettbewerbs schützen.

\12 Als "Unternehmen" < 4> bezeichnet dies Gesetz natürliche und juristische Personen und sonstige Organisationen, die sich mit Produktion oder Vertrieb von Waren befassen oder Dienstleistungen erbringen.

Als den "betroffenen Markt" bezeichnet dies Gesetz bei Unternehmen, die innerhalb einer bestimmten Zeit mit bestimmten Waren oder Dienstleistungen (im folgenden sämtlich als "Waren" bezeichnet) im Wettbewerb stehen, den Bereich der Waren und das geographische Gebiet des Wettbewerbs.

2. Kapitel: Monopolvereinbarungen

\13 Im Wettbewerb miteinander stehenden Unternehmen ist es verboten, Monopolvereinbarungen zu treffen, die

1. Warenpreise festlegen oder ändern,

2. Produktions- oder Absatzmengen von Waren einschränken,

3. Absatz- oder Beschaffungsmärkte aufteilen,

4. den Ankauf neuer Techniken und neuer Anlagen einschränken oder die Erschließung neuer Techniken und neuer Produkte einschränken,

5. [die Beteiligten] zum Boykott von Geschäften [mit Dritten] verbinden,

6. sonstige Vereinbarungen sind, für die vom Antimonopolrecht-Vollzugsorgan des Staatsrats festgestellt worden ist, daß es sich um Monopolvereinbarungen handelt.

Als Monopolvereinbarungen bezeichnet dies Gesetz Wettbewerb ausschließende oder einschränkende Vereinbarungen, Beschlüsse und andere abstimmende Handlungen.

\14 Unternehmen ist es verboten, mit Geschäftspartnern Monopolvereinbarungen zu treffen, die

1. Preise für den Weiterverkauf von Waren an Dritte festlegen,

2. Mindestpreise für den Weiterverkauf von Waren an Dritte begrenzen,

3. sonstige Vereinbarungen sind, für die vom Antimonopolrecht-Vollzugsorgan des Staatsrats festgestellt worden ist, daß es sich um Monopolvereinbarungen handelt. 
$\int 15 \iint 13$ und 14 werden nicht angewandt, wenn die Unternehmen beweisen können, daß ihre Vereinbarungen getroffen worden sind, um

1. Techniken zu verbessern, die Erschließung neuer Produkte zu untersuchen, oder

2. zur Erhöhung der Produktqualität, Kostensenkung oder Effizienzsteigerung Produkttypen und -normen zu vereinheitlichen oder Arbeitsteilung durch Spezialisierung durchzuführen, oder

3. die Betriebseffizienz mittlerer und kleiner Unternehmen zu erhöhen, die Wettbewerbsfähigkeit mittlerer und kleiner Unternehmen zu stärken, oder

4. dem Allgemeininteresse $<2>$ zu dienen, wie um Energie zu sparen, die Umwelt zu schützen, in Katastrophen zu helfen, oder

5. wegen einer wirtschaftlichen Krise erheblich gesunkenen Absatz oder deutliche Überproduktion abzumildern, oder

6. um angemessene Gewinne im Außenhandel und in der wirtschaftlichen Zusammenarbeit mit dem Ausland zu gewährleisten, und

7. unter anderen von Gesetzen oder vom Staatsrat bestimmten Umständen.

Wenn in den Fällen der Nrn. 1 bis 5 des vorigen Absatzes $\iint 13$ und 14 nicht angewandt werden [sollen], müssen die Unternehmen auch beweisen, daß die von ihnen getroffenen Vereinbarungen den Wettbewerb auf dem betroffenen Markt nicht erheblich beeinträchtigen können, und daß man die Verbraucher am daraus erzielten Gewinn teilhaben lassen kann.

$\int 16$ Branchenverbände dürfen nicht organisieren, daß Unternehmen ihrer Branche in diesem Kapitel verbotene monopolistische Handlungen betreiben.

3. Kapitel: Mißbrauch einer marktbeherrschenden Stellung

\17 Einem Unternehmen, das eine marktbeherrschende Stellung innehat, ist es verboten, seine marktbeherrschende Stellung zu mißbrauchen, indem es

1. Waren zu unfair hohen Preisen verkauft oder zu unfair niedrigen Preisen ankauft,

2. ohne angemessenen Grund Waren zu Preisen unter den Selbstkosten verkauft,

3. ohne angemessenen Grund Geschäfte mit Geschäftspartnern ablehnt,

4. ohne angemessenen Grund Geschäftspartner darauf beschränkt, nur mit ihm oder von ihm bestimmten Unternehmen Geschäfte zu betreiben,

5. ohne angemessenen Grund Waren miteinander gekoppelt verkauft oder bei Geschäften andere unvernünftige Geschäftsbedingungen stellt,

6. ohne angemessenen Grund Geschäftspartner, bei denen die gleichen Bedingungen gegeben sind, bei den Preisen oder anderen Geschäftsbedingungen unterschiedlich behandelt,

7. in einer anderen Weise verfährt, für die vom Antimonopolrecht-Vollzugsorgan des Staatsrats festgestellt worden ist, daß es sich um Mißbrauch einer marktbeherrschenden Stellung handelt.

Als "marktbeherrschende Stellung" bezeichnet dies Gesetz die Marktstellung, 
die ein Unternehmen innehat, das auf dem betroffenen Markt Warenpreise oder -mengen oder andere Geschäftsbedingungen kontrollieren oder die Fähigkeit anderer Unternehmen, auf den betroffenen Markt zu kommen, behindern, beeinflussen kann $<5>$.

$\int 18$ Die Feststellung, daß ein Unternehmen eine marktbeherrschende Stellung innehat, muß auf den folgenden Punkten beruhen:

1. Dem Marktanteil dieses Unternehmens am betroffenen Markt und den Wettbewerbsverhältnissen auf dem betroffenen Markt,

2. der Fähigkeit dieses Unternehmens, Absatz- und Beschaffungsmärkte zu kontrollieren,

3. der Finanzkraft dieses Unternehmens und seinen technischen Bedingungen,

4. dem Grad, in dem andere Unternehmen im Geschäftsverkehr von diesem Unternehmen abhängig sind,

5. der Schwierigkeit oder Leichtigkeit des Zutritts anderer Unternehmen zum betroffenen Markt,

6. anderen Punkten, die mit der Feststellung der marktbeherrschenden Stellung dieses Unternehmens zu tun haben.

\19 Liegt einer der folgenden Umstände vor, so kann geschlossen werden, daß die [betreffenden] Unternehmen eine marktbeherrschende Stellung innehaben:

1. Wenn ein Unternehmen auf dem betroffenen Markt einen Marktanteil von 50\% erreicht,

2. wenn zwei Unternehmen zusammen auf dem betroffenen Markt einen Marktanteil von zwei Dritteln erreichen,

3. wenn drei Unternehmen zusammen auf dem betroffenen Markt einen Marktanteil von drei Vierteln erreichen.

Wenn in den Fällen der Nrn. 2 und 3 des vorigen Absatzes ein eingerechnetes Unternehmen einen Marktanteil unter einem Zehntel hat, muß nicht geschlossen werden, daß dies Unternehmen eine marktbeherrschende Stellung innehat. $<6>$

Wenn ein Unternehmen, bei dem geschlossen wird, daß es eine marktbeherrschende Stellung innehat, Beweise dafür hat, daß es keine marktbeherrschende Stellung innehat, muß nicht festgestellt werden, daß dies Unternehmen eine marktbeherrschende Stellung innehat.

4. Kapitel: Unternehmenszusammenschluß

\20 Mit Unternehmenszusammenschluß ist gemeint:

1. die Fusion von Unternehmen;

2. daß Unternehmen mit dem Erwerb von Anteilsrechten oder Vermögen die Kontrolle über andere Unternehmen erlangen;

3. daß Unternehmen durch Verträge oder auf andere Weise die Kontrolle über andere Unternehmen erlangen oder auf andere Unternehmen einen bestimmenden Einluß ausüben können. 
\21 Wenn ein Unternehmenszusammenschluß eine vom Staatsrat bestimmte Meldegrenze erreicht, müssen die Unternehmen dem Antimonopolrecht-Vollzugsorgan des Staatsrats vorher Meldung erstatten; ungemeldet darf der Unternehmenszusammenschluß nicht vollzogen werden.

$\int 22$ Wenn bei einem Unternehmenszusammenschluß einer der folgenden Umstände vorliegt, braucht er dem Antimonopolrecht-Vollzugsorgan des Staatsrats nicht gemeldet zu werden:

1. Wenn eines der beteiligten Unternehmen an jedem der anderen Unternehmen mindestens 50\% der stimmberechtigten Anteile oder des Vermögens besitzt;

2. wenn ein und dasselbe nicht beteiligte Unternehmen mindestens $50 \%$ der stimmberechtigten Anteile oder des Vermögens jedes der beteiligten Unternehmen besitzt.

$\int 23$ Wenn Unternehmen dem Antimonopolrecht-Vollzugsorgan des Staatsrats einen Zusammenschluß melden, müssen sie die folgenden Schriftstücke und Unterlagen einreichen:

1. die schriftliche Meldung,

2. eine Erläuterung der Auswirkungen des Zusammenschlusses auf den Wettbewerb auf dem betroffenen Markt, 3. die Zusammenschlußvereinbarung,

4. die Finanz- und Buchführungsberichte der am Zusammenschluß beteiligten Unternehmen für das vorangegangene Buchführungsjahr, nach Rechnungsprüfung der Berichte durch Buchprüfungsbüros,

5. andere vom Antimonopolrecht-Vollzugsorgan des Staatsrats bestimmte Schriftstücke und Unterlagen.

Die schriftliche Meldung muß die Bezeichnungen der am Zusammenschluß beteiligten Unternehmen, ihre Sitze, ihre Geschäftsbereiche, das für den Vollzug des Zusammenschlusses vorgesehene Datum und andere vom Antimonopolrecht-Vollzugsorgan des Staatsrats bestimmte Punkte enthalten.

$\int 24$ Wenn von den Unternehmen eingereichte Schriftstücke und Unterlagen unvollständig sind, müssen sie in einer vom Antimonopolrecht-Vollzugsorgan des Staatsrats bestimmten Frist ergänzt werden. Wenn die Unternehmen sie nicht fristgemäß ergänzen, gilt die Meldung als nicht erstattet.

$\int 25$ Innerhalb von 30 Tagen ab dem Tag, an dem das Antimonopolrecht-Vollzugsorgan des Staatsrats die von den Unternehmen eingereichten und $\int 23$ entsprechenden Schriftstücke und Unterlagen erhalten hat, unterzieht es den gemeldeten Zusammenschluß einer ersten Prüfung, beschließt, ob es eine weitere Prüfung durchführt und teilt das den Unternehmen schriftlich mit. Bevor das Antimonopolrecht-Vollzugsorgan des Staatsrats den Beschluß erlassen hat, dürfen die Unternehmen den Zusammenschluß nicht vollziehen.

Wenn das Antimonopolrecht-Vollzugsorgan des Staatsrats beschließt, keine 
weitere Prüfung durchzuführen oder innerhalb der Frist keinen Beschluß erläßt, können die Unternehmen den Zusammenschluß vollziehen.

$\int 26$ Wenn das Antimonopolrecht-Vollzugsorgan des Staatsrats beschließt, eine weitere Prüfung durchzuführen, muß es innerhalb von 90 Tagen ab dem Tag des Beschlusses die Prüfung abschließen und beschließen, ob es den Unternehmenszusammenschluß verbietet oder nicht und dies schriftlich den Unternehmen mitteilen. Beschließt es, den Zusammenschluß zu verbieten, so muß es die Gründe erläutern. Solange die Prüfung dauert, dürfen die Unternehmen den Zusammenschluß nicht vollziehen.

Wenn einer der folgenden Umstände vorliegt, kann das Antimonopolrecht-Vollzugsorgan des Staatsrats mit schriftlicher Mitteilung an die Unternehmen die im vorigen Absatz bestimmte Prüfungsfrist um bis zu 60 Tage verlängern:

1. Wenn die Unternehmen der Verlängerung der Prüfungsfrist zustimmen,

2. wenn von den Unternehmen eingereichte Schriftstücke und Unterlagen inkorrekt sind und näher geprüft werden müssen,

3. wenn sich nach der Meldung durch die Unternehmen die betreffenden Umstände erheblich ändern.

Wenn das Antimonopolrecht-Vollzugsorgan des Staatsrats nicht fristgemäß seinen Beschluß faßt, können die Unternehmen den Zusammenschluß vollziehen.

\27 Bei der Prüfung des Unternehmenszusammenschlusses müssen die folgenden Punkte berücksichtigt werden:

1. Die Marktanteile der beteiligten Unternehmen am betroffenen Markt und ihre Fähigkeit, den Markt zu kontrollieren,

2. der Grad der Konzentration auf dem betroffenen Markt,

3. die Auswirkungen des Unternehmenszusammenschlusses auf den Marktzugang und auf den technischen Fortschritt,

4. die Auswirkungen des Unternehmenszusammenschlusses auf die Verbraucher und auf betroffene andere Unternehmen,

5. die Auswirkungen des Unternehmenszusammenschlusses auf die Entwicklung der Volkswirtschaft, 6. was sich sonst auf den Marktwettbewerb auswirkt und nach Ansicht des Antimonopolrecht-Vollzugsorgans des Staatsrats berücksichtigt werden muß.

\28 Wenn der Unternehmenszusammenschluß dazu führt oder führen kann, daß Wettbewerb ausgeschlossen oder eingeschränkt wird, muß das Antimonopolrecht-Vollzugsorgan des Staatsrats beschließen, den Unternehmenszusammenschluß zu verbieten. Wenn die Unternehmen jedoch beweisen können, daß die dem Wettbewerb günstigen Auswirkungen des Zusammenschlusses deutlich größer sind als die ungünstigen, oder daß [der Zusammenschluß] dem Allgemeininteresse <2>entspricht, kann das Antimonopolrecht-Vollzugsorgan des Staatsrats beschließen, den 
Unternehmenszusammenschluß nicht zu verbieten.

\29 Das Antimonopolrecht-Vollzugsorgan des Staatsrats kann einen nicht verbotenen Unternehmenszusammenschluß einschränkende Bedingungen beschließen, um ungünstige Auswirkungen des Zusammenschlusses auf den Wettbewerb zu mindern.

$\int 30$ Das Antimonopolrecht-Vollzugsorgan des Staatsrats muß Beschlüsse, mit denen ein Unternehmenszusammenschluß verboten oder einschränkenden Bedingungen unterworfen wird, unverzüglich der Allgemeinheit bekanntgeben.

\31 Wenn jemand mit ausländischem Kapital Fusionen mit Unternehmen im Gebiet durchführt oder sich auf andere Weise mit ausländischem Kapital an einem Unternehmenszusammenschluß beteiligt, und dies die Staatssicherheit berührt, muß nicht nur nach vorliegendem Gesetz der Unternehmenszusammenschluß geprüft werden, sondern es müssen auch nach den einschlägigen staatlichen Vorschriften [die Auswirkungen auf] die Staatssicherheit geprüft werden.

5. Kapitel: Mißbrauch von Verwaltungsmacht, um Wettbewerb auszuschließen oder einzuschränken

\32 Verwaltungsbehörden und von Gesetzen und anderen Rechtsnormen ermächtigte, Funktionen beim Management öffentlicher Angelegenheiten ausübende Organisationen dürfen Verwaltungsmacht nicht mißbrauchen, um direkt oder indirekt den Vertrieb, Kauf oder Gebrauch der Waren von ihnen bestimmter Unternehmen durch Einheiten oder einzelne einzuschränken.

$\int 33$ Verwaltungsbehörden und von Gesetzen und anderen Rechtsnormen ermächtigte, Funktionen beim Management öffentlicher Angelegenheiten ausübende Organisationen dürfen nicht in Mißbrauch von Verwaltungsmacht mit den folgenden Handlungen den freien Warenverkehr zwischen [chinesischen] Territorien behindern:

1. auswärtige Waren benachteiligende Gebührengegenstände festsetzen, auswärtige Waren benachteiligende Gebührensätze anwenden oder auswärtige Waren benachteiligende Preise bestimmen $<7>$,

2. für auswärtige Waren andere technische Anforderungen und Prüfungsnormen bestimmen als für gleichartige örtliche Waren, oder sie durch technische Maßnahmen, wie erneute Prüfung oder erneute Dokumentation, benachteiligen, um das Vordringen auswärtiger Waren auf den örtlichen Markt einzuschränken $<8>$,

3. speziell für auswärtige Waren Verwaltungslizenzen vorsehen, um ihr Vordringen auf den örtlichen Markt einzuschränken $<9>$,

4. Kontrollstellen einrichten oder andere Verfahren verwenden, um das Hereinkommen auswärtiger oder das Hinausgelangen örtlicher Waren zu 
behindern $<10>$,

5. mit anderen Handlungen den freien Warenverkehr zwischen [chinesischen] Territorien behindern.

\34 Verwaltungsbehörden und von Gesetzen und anderen Rechtsnormen ermächtigte, Funktionen beim Management öffentlicher Angelegenheiten ausübende Organisationen dürfen nicht in Mißbrauch von Verwaltungsmacht die Teilnahme auswärtiger Unternehmen an örtlichen Ausschreibungen durch sie einseitig benachteiligende Materialanforderungen oder Bewertungsmaßstäbe oder dadurch, daß Daten nicht nach dem Recht bekanntgemacht werden oder auf andere Weise einschränken oder ausschließen $<11>$.

$\int 35$ Verwaltungsbehörden und von Gesetzen und anderen Rechtsnormen ermächtigte, Funktionen beim Management öffentlicher Angelegenheiten ausübende Organisationen dürfen nicht in Mißbrauch von Verwaltungsmacht dadurch, daß sie auswärtige Unternehmen und örtliche Unternehmen nicht gleich behandeln, oder auf andere Weise einschränken oder ausschließen, daß auswärtige Unternehmen örtlich investieren oder Zweigorgane errichten $<12>$.

\36 Verwaltungsbehörden und von Gesetzen und anderen Rechtsnormen ermächtigte, Funktionen beim Management öffentlicher Angelegenheiten ausübende Organisationen dürfen nicht in Mißbrauch von Verwaltungsmacht Unternehmen zu Handlungen zwingen, die nach dem vorliegenden Gesetz monopolistische Handlungen sind.

$\int 37$ Verwaltungsbehörden dürfen nicht in Mißbrauch von Verwaltungsmacht Vorschriften mit Wettbewerb ausschließendem oder einschränkendem Inhalt festsetzen.

6. Kapitel: Untersuchung von als monopolistisch verdächtigen Handlungen

\38 Antimonopolrecht-Vollzugsorgane untersuchen nach dem Recht als monopolistisch verdächtige Handlungen.

Jede Einheit und jeder einzelne ist $\mathrm{zu}$ Meldungen als monopolistisch verdächtiger Handlungen an Antimonopolrecht-Vollzugsorgane berechtigt. Die Antimonopolrecht-Vollzugsorgane müssen die Meldenden geheimhalten.

Wenn eine Meldung schriftlich erstattet wird, und einschlägige Tatsachen und Beweise vorgelegt werden, müssen die Antimonopolrecht-Vollzugsorgane die erforderlichen Untersuchungen durchführen.

\39 Antimonopolrecht-Vollzugsorgane können bei der Untersuchung als monopolistisch verdächtiger Handlungen folgende Maßnahmen ergreifen:

1. Zu Nachprüfungen Betriebsstätten untersuchter Unternehmen und sonst betroffene Örtlichkeiten betreten, 
2. untersuchte Unternehmen, materiell Interessierte und sonst betroffene Einheiten und einzelne befragen und von ihnen verlangen, daß sie einschlägige Umstände erläutern,

3. einschlägige Einzelnachweise, Vereinbarungen, Buchführung, betriebliche Briefe und Telegramme, elektronische Daten und andere Schriftstücke und Unterlagen der untersuchten Unternehmen, materiell Interessierten und sonst betroffener Einheiten und einzelner durchsehen und kopieren,

4. einschlägige Beweise versiegeln und pfänden,

5. Bankkonten der Unternehmen überprüfen und Fragen dazu stellen.

Maßnahmen nach dem vorigen Absatz müssen dem hauptsächlichen verantwortlichen Leiter des Antimonopolrecht-Vollzugsorgans schriftlich gemeldet und [von ihm] genehmigt werden.

\40 Eine Untersuchung als monopolistisch verdächtiger Handlungen durch Antimonopolrecht-Vollzugsorgane muß von mindestens zwei Rechtsvollziehern ausgeführt werden, die Rechtsvollzieherausweise vorzeigen müssen.

Über Befragungen und Untersuchungen müssen die Rechtsvollzieher ein Protokoll anfertigen, das von den Befragten bzw. Untersuchten unterschrieben wird.

\41 Antimonopolrecht-Vollzugsorgane und ihre Mitarbeiter haben die Pflicht, über Geschäftsgeheimnisse, die sie beim Rechtsvollzug erfahren, Verschwiegenheit zu bewahren.

$\int 42$ Untersuchte Unternehmen, materiell Interessierte und sonst betroffene Einheiten und einzelne müssen mit Antimonopolrecht-Vollzugsorganen, die nach dem Recht ihre Amtsaufgaben ausüben, kooperieren und dürfen sich deren Untersuchungen nicht verweigern oder sie behindern.

$\int 43$ Untersuchte Unternehmen und materiell Interessierte haben das Recht, Einwände zu erheben. Die Antimonopolrecht-Vollzugsorgane müssen von den untersuchten Unternehmen und von materiell Interessierten vorgebrachte Tatsachen, Gründe und Beweise überprüfen.

$\int 44$ Wenn ein Antimonopolrecht-Vollzugsorgan als monopolistisch verdächtige Handlungen nach Untersuchung und Überprüfung für monopolistisch hält, muß es nach dem Recht einen Beschluß zur Regelung der Sache fassen, den es der Allgemeinheit bekanntgeben kann.

\45 Wenn ein Antimonopolrecht-Vollzugsorgan als monopolistisch verdächtige Handlungen untersucht, und das untersuchte Unternehmen zusagt, innerhalb einer vom Antimonopolrecht-Vollzugsorgan zugestandenen Frist konkrete Maßnahmen zu ergreifen, um die Folgen dieses Handelns zu beheben, kann das Antimonopolrecht-Vollzugsorgan beschließen, die Untersuchung zu unterbrechen. 
Der Unterbrechungsbeschluß muß angeben, was das untersuchte Unternehmen konkret zugesagt hat.

Wenn ein Antimonopolrecht-Vollzugsorgan beschließt, eine Untersuchung zu unterbrechen, muß es beaufsichtigen, wie das Unternehmen seine Zusage ausführt. Wenn das Unternehmen seine Zusage ausführt, kann das Antimonopolrecht-Vollzugsorgan beschließen, die Untersuchung zu beenden.

Wenn einer der folgenden Umstände vorliegt, muß das Antimonopolrecht-Vollzugsorgan die Untersuchung wiederaufnehmen:

1. Wenn das Unternehmen seine Zusage nicht erfüllt,

2. wenn sich die Tatsachen, die dem Unterbrechungsbeschluß zugrundeliegen, erheblich ändern,

3. wenn der Unterbrechungsbeschluß auf unvollständigen oder unwahren Angaben des Unternehmens beruht.

\section{Kapitel: Rechtliche Verantwortung}

\46 Wenn ein Unternehmen entgegen diesem Gesetz eine Monopolvereinbarung trifft und ausführt, weist das Antimonopolrecht-Vollzugsorgan es an, die rechtswidrigen Handlungen einzustellen, zieht das rechtswidrig Erlangte ein und verhängt eine Geldbuße in Höhe von einem bis 10\% des letzten Jahresumsatzes; ist die getroffene Monopolvereinbarung noch nicht ausgeführt worden, kann eine Geldbuße von bis zu 500.000 Yuan verhängt werden.

Wenn ein Unternehmen von sich aus dem Antimonopolrecht-Vollzugsorgan die Umstände des Abschlusses einer Monopolvereinbarung mitteilt und wichtige Beweise zur Verfügung stellt, kann das Antimonopolrecht-Vollzugsorgan unter Berücksichtigung der Umstände die gegen dies Unternehmen zu verhängende Geldbuße ermäßigen oder erlassen.

Wenn Branchenverbände entgegen diesem Gesetz organisieren, daß Unternehmen ihrer Branche eine Monopolvereinbarung treffen, kann das Antimonopolrecht-Vollzugsorgan eine Geldbuße von bis zu 500.000 Yuan verhängen; bei schwerwiegenden Umständen kann die Registerverwaltungsbehörde für gesellschaftliche Körperschaften nach dem Recht die Registrierung [des Verbands] löschen.

$\int 47$ Wenn ein Unternehmen entgegen diesem Gesetz eine marktbeherrschende Stellung mißbraucht, weist das Antimonopolrecht-Vollzugsorgan es an, die rechtswidrigen Handlungen einzustellen, zieht das rechtswidrig Erlangte ein und verhängt eine Geldbuße in Höhe von einem bis 10\% des letzten Jahresumsatzes.

$\int 48$ Wenn Unternehmen entgegen diesem Gesetz einen Zusammenschluß vollziehen, weist das Antimonopolrecht-Vollzugsorgan des Staatsrats sie an, den Vollzug des Zusammenschlusses einzustellen und innerhalb bestimmter Fristen über Anteile und Vermögen zu verfügen, Betrieb zu übertragen und sonst erforderliche Maßnahmen zu ergreifen, um den Zustand vor dem 
Zusammenschluß wiederherzustellen, und es kann eine Geldbuße von bis zu 500.000 Yuan verhängen.

$\int 49$ Wenn Antimonopolrecht-Vollzugsorgane Geldbußen nach den $\iint 46,47$ und 48 verhängen, müssen sie bei der Festsetzung des konkreten Betrags Dinge wie Natur und Ausmaß der rechtswidrigen Handlungen und die Dauer der Zeit, während der sie fortgesetzt wurden, berücksichtigen.

$\int 50$ Wenn Unternehmen mit monopolistischen Handlungen andere Personen schädigen, haften sie nach dem Recht zivilrechtlich.

$\int 51$ Wenn Verwaltungsbehörden und von Gesetzen und anderen Rechtsnormen ermächtigte, Funktionen beim Management öffentlicher Angelegenheiten ausübende Organisationen Verwaltungsmacht mißbrauchen, um Wettbewerb auszuschließen oder einzuschränken, weisen höhere Behörden sie an, dies zu korrigieren; direkt verantwortliches vorgesetztes und anderes direkt verantwortliches Personal wird nach dem Recht gemaßregelt. Antimonopolrecht-Vollzugsorgane können den betreffenden höheren Behörden Regelungen [solcher Fälle] nach dem Recht vorschlagen.

Wenn in Gesetzen oder anderen Rechtsnormen anders geregelt ist, wie gegenüber Wettbewerb einschränkendem oder ausschließendem Mißbrauch von Verwaltungsmacht durch Verwaltungsbehörden und durch von Gesetzen und anderen Rechtsnormen ermächtigte, Funktionen beim Management öffentlicher Angelegenheiten ausübende Organisationen zu verfahren ist, gelten diese anderen Vorschriften.

$\int 52$ Wenn ein Antimonopolrecht-Vollzugsorgan nach dem Recht Prüfungen und Untersuchungen durchführt, und jemand ihm dazu Unterlagen und Informationen verweigert oder falsche Unterlagen und Informationen liefert oder Beweise verheimlicht, vernichtet oder anderswohin verbringt oder Untersuchungen auf andere Weise verweigert oder behindert, weist ihn das Antimonopolrecht-Vollzugsorgan an, dies zu korrigieren; ferner kann das Antimonopolrecht-Vollzugsorgan [in diesen Fällen] gegen einen einzelnen eine Geldbuße von bis zu 20.000 Yuan, gegen eine Einheit eine Geldbuße von bis zu 200.000 Yuan verhängen; bei schwerwiegenden Umständen kann es gegen einen einzelnen eine Geldbuße zwischen 20.000 und 100.000 Yuan, gegen eine Einheit eine Geldbuße zwischen 200.000 und 1 Mio. Yuan verhängen; bilden die Handlungen eine Straftat, so wird nach dem Recht die strafrechtliche Verantwortung verfolgt.

$\int 53$ Wer sich einem Beschluß des Antimonopolrecht-Vollzugsorgans nach \ 28 oder \29 nicht unterwerfen will, kann zunächst nach dem Recht $<13>$ erneute Verwaltungsberatung beantragen; will er sich dem Beschluß nach erneuter Verwaltungsberatung nicht unterwerfen, so kann er nach dem Recht 
Verwaltungsklage erheben.

Wer sich einem Beschluß des Antimonopolrecht-Vollzugsorgans nach anderen als den im vorigen Absatz genannten Vorschriften nicht unterwerfen will, kann nach dem Recht erneute Verwaltungsberatung beantragen oder Verwaltungsklage erheben.

$\int 54$ Wenn Mitarbeiter eines Antimonopolrecht-Vollzugsorgans ihre Amtsgewalt mißbrauchen, ihr Amt vernachlässigen, mit unsauberen Mitteln private Interessen verfolgen oder Geschäftsgeheimnisse, die sie beim Rechtsvollzug erfahren, durchsickern lassen, und dies eine eine Straftat bildet, wird nach dem Recht die strafrechtliche Verantwortung verfolgt; bildet es noch keine Straftat, so werden sie nach dem Recht gemaßregelt.

8. Kapitel: Ergänzende Regeln

$\int 55$ Auf Handlungen, mit denen Unternehmen nach den Gesetzen und Verwaltungsrechtsnormen zu geistigen Eigentumsrechten ein geistiges Eigentumsrecht ausüben, wird dies Gesetz nicht angewandt; es wird jedoch angewandt, wenn ein Unternehmen ein geistiges Eigentumsrecht mißbraucht, um Wettbewerb auszuschließen oder einzuschränken.

\56 Auf Verbindungen oder abstimmende Handlungen landwirtschaftlicher Produzenten oder dörflicher Wirtschaftsorganisationen bei Betriebsaktivitäten wie Produktion, Bearbeitung, Absatz, Transport und Lagerung landwirtschaftlicher Produkte wird dies Gesetz nicht angewandt.

\57 Dies Gesetz wird ab dem 1.8.2008 angewandt.

Quelle: www.gov.cn/ziliao/flfg/2007-08/30/content_732591.htm

\section{Anmerkungen:}

$<1>$ Am 17.10.1980 erließ der Staatsrat "Vorläufige Bestimmungen zur Entwicklung und zum Schutz des sozialistischen Wettbewerbs" (deutsch in RIW/AWD 1987/4.261). Sie ergingen, nachdem die Partei "Autonomie" der Staatsunternehmen und "Ausgleich durch den Markt" dekretiert hatte und waren das erste Wettbewerbsrecht der Volksrepublik, denn zur Erfüllung der Staatspläne, Verbesserung der Betriebsführung, Verbesserung der Produktqualität, Kostensenkung usw.usw. sollte nun "Wettbewerb entfaltet", sollten wirtschaftliche Gesetzmäßigkeiten genutzt werden, um eine "gesunde Entwicklung des Wettbewerb zu lenken". Dazu wurde u.a. die Abriegelung der Unternehmen unter Verwaltungsgebieten und Branchenbehörden gegen Konkurrenz der Unternehmen anderer Territorien und Behörden. Dabei gingen die "Vorläufigen Bestimmungen" sogar weiter als das vorliegende Gesetz: Sie enthielten keine Ausnahmen für Staatsunternehmen - denn von Kleinbetrieben abgesehen gab es 
damals nur Staatsunternehmen. Die "Vorläufigen Bestimmungen" waren jedoch nur fromme Wünsche. Es gab keine Handhabe, um sie durchzusetzen.

Mit der wachsenden Unabhängigkeit der Staatsunternehmen ging es auf dem Markt immer lebhafter zu, immer mehr aber mißbrauchten auch die vorgesetzten Behörden der Unternehmen ihre Macht, um ihre Unternehmen gegen die Konkurrenz der Unternehmen anderer Behörden zu schützen, also um zu tun, was die "Vorläufigen Bestimmungen" verboten hatten: um die Unternehmenssysteme von Branchen- und Territorialbehörden abzuschotten. Das fiel allmählich auch dem Publikum unangenehm auf, das, eben noch glücklich, Versorgungsengpässen der Planwirtschaft zu entrinnen, wieder auf Lokalprodukte beschränkt wurde, etwa in Peking ausgerechnet im heißen Sommer 1985 kein Bier aus Qingdao oder der Inneren Mongolei bekam, sondern nur die dann auch noch knappe lokale Plörre.

Man sah sich an, was anderswo in solcher Lage getan wurde, also marktwirtschaftliches Wettbewerbsrecht, und arbeitete 1986 einen ersten Entwurf eines Wettbewerbsgesetzes aus, der nach taiwanesischem Vorbild Vorschriften gegen unlauteren Wettbewerb und gegen Monopole zusammenfaßte. Nicht nur dies Vorbild, die ganze Richtung ging den Ideologen jedoch zu weit. Zustande kam schließlich nur ein Gesetz gegen unlauteren Wettbewerb, 2.9.93/1. Es enthielt aber auch Antimonopolrecht: Es verbot in $\iint 6$ und 7 Monopolunternehmen, insbesondere staatlichen Monopolunternehmen, und Behörden, ihre Marktstellung bzw. Verwaltungsmacht zu nutzen, um ihre Konkurrenz und die Konkurrenz ihrer Unternehmen auszuschalten.

Das war ein unerwartet guter Anfang, denn dies war der Bereich, in dem Kartellrecht praktisch am dringendsten gebraucht wurde, der aber auch politisch besonders empfindlich war. Hier hätte die zuständige Behörde mit Verwaltungsverordnungen und Gesetzesentwürfen das Kartellrecht rasch weiterentwickeln können - wäre man sich nur darüber einig gewesen, wer zuständige Behörde sein sollte. Zwei zentrale Behörden stritten sich um diese Aufgabe und tun das noch heute.

Das Staatliche Industrie- und Handelsverwaltungshauptamt (IHVA, Netzseite: www.saic.gov.cn) schien von Natur aus zum Kartellamt berufen. Denn seine Hauptaufgabe war die Marktkontrolle. Nur waren die Märkte, die es zu kontrollieren hatte, ursprünglich nur die kleinen freien Märkte, auf denen insbesondere Bauern ihre "freien Spitzen" anboten, überplanmäßige Produkte, die nicht an den staatlichen Monopolhandel abgegeben werden mußten. Händler auf solchen Märkten ließen sich von den auf Motorrädern herumdonnernden IHVA-Kontrolleuren einschüchtern. Aber das IHVA hatte kaum die Macht, sich nach $\iint 6$ und 7 des Gesetzes gegen unlauteren Wettbewerb mit mächtigen Staatsbetrieben und zentralen Branchenbehörden anzulegen. Eben um Macht zu gewinnen, hätte es freilich gern auch diese Aufgabe übernommen.

Macht, gerade gegenüber den großen Staatsunternehmen und ihren Behörden, hatte dagegen der Rivale des IHVA, die Staatliche Preiskommission, längst in jahrzehntelangem Kämpfen errungen. Preise wurden in der Planwirtschaft vom 
Staat bestimmt, eben von der Plankommission oder ihren Unterbehörden, aber nach Vorgaben der Unternehmen. Die Unternehmen mußten sich dabei nach ihren Selbstkosten richten, aber "Kosten sind das am leichtesten zu produzierende Produkt" (Mestmäcker); die Unternehmen beschwindelten die Kommission, wo sie nur konnten, und die Kommission blieb deshalb den Unternehmen immer dicht auf den Fersen und wußte sehr genau über deren Machenschaften Bescheid. Dies Wissen und die sich daraus ergebende Macht über und Gegnerschaft gegenüber den Großunternehmen waren genau das, was ein Kartellamt brauchte. Deshalb wollte auch die Preiskommission diese Aufgabe haben.

Mit dem Vergehen der Planwirtschaft ist die Preiskommission verschwunden, in Wahrheit aber zu einer Abteilung der Plankommission geworden, die inzwischen auch nicht mehr so heißt, sondern "Staatskommission für Entwicklung und Reform" (Netzseite: http://fgs.ndrc.gov.cn/), in der das "Preisamt" weiterhin eine besonders aktive Abteilung ist. Noch die Preiskommission hatte die Entwürfe für das Preisgesetz, 29.12.97/1, ausgearbeitet, das in $\int 14$ Nr. 1 Preiskartelle verbietet und in $\int 18$ der Regierung nur in eng begrenztem Rahmen gestattet, Preise festzusetzen.

IHVA einerseits, Preiskommission/Preisamt (PA) andererseits haben dann zu diesen Gesetzen Verwaltungsverordnungen entworfen, insbesondere anscheinend das IHVA 21.4.01/1 gegen die Abschottung von Verwaltungsgebieten, das PA 18.6.03/1 gegen Preismonopolisierung. Beide haben sich natürlich auch an den Arbeiten an den Etwürfen zum Antimonopolgesetz beteiligt.

So konnte auch in der schließlich verabschiedeten Fassung dieses Gesetzes nicht bestimmt werden, wer es ausführen soll. Der Streit zwischen IHVA und PA hat das verhindert; das Gesetz spricht nur vage von "Antimonopolrecht-Vollzugsorganen". Wohl aus dem gleichen Grund fehlen auch Vorschriften zur Organisation der Antimonopolkommission nach $\int 9$. Ohne Vorschriften zu diesen Organen kann das Gesetz aber nicht angewandt werden. Wohl deshalb gilt es erst ab August 2008.

Vielleicht läßt sich der Streit aber bis dahin beilegen. Gravierender ist ein anderes Problem. Das Gesetz ist stark vom Preisgesetz, 29.12.97/1 und noch mehr von den Ausführungsvorschriften dazu in 18.6.03/1 beeinflußt. Diese Vorschriften verfolgen ein klar marktwirtschaftliches Wirtschaftsmodell. Sie gestehen Staatsunternehmen keine Sonderrolle zu, sondern richten sich gegen alle marktbeherrschenden Unternehmen und alle Preiskartelle. Staatlich festgesetzte Preise erlaubt das Preisgesetz in \ 18 nur "1.für eine ganz geringe Zahl von Waren von außerordentlicher Bedeutung für die volkswirtschaftliche Entwicklung und das Leben der Bevölkerung; 2.für eine kleine Zahl von Waren knapper Ressourcen; 3.für Waren, die in natürlichen Monopolen vertrieben werden; 4.für die Preise bei wichtigen öffentlichen Institutionen; 5.für wichtige Dienstleistungen für das Gemeinwohl", also in eng begrenzten Ausnahmefällen bei Allgemeininteresse und bei unvermeidlichen Monopolen.

Diese engen Grenzen für Ausnahmen vom Wettbewerbsrecht hat das vorliegende Gesetz aber nicht übernommen. In seinem $\int 7$ zeigt sich ein anderes 
Wirtschaftsmodell, das der "Leitlinien für Staatskapital und Staatsunternehmen" (5.12.06/1) der Staatsvermögenskommission. Sie unterscheiden zwischen Unternehmen in "wichtigen Branchen und Schlüsselbereichen" (kurz: Schlüsselindustrien) und anderen Unternehmen. $\mathrm{Zu}$ den Schlüsselindustrien gehören insbesondere die Staatsicherheit berührende Unternehmen, wichtige Infrastrukturanlagen und Bergwerke, Lieferanten wichtiger öffentliche Produkte und Dienstleistungen, Telekommunikation, Stromversorgung, Zivilluftfahrt, Kernunternehmen in Industrien, die "Stützpfeiler der Wirtschaft" darstellen, Industrien mit neuer Hochtechnologie - also ein Bereich, dessen vage Grenzen recht beliebig gezogen werden können. Nicht nur, wie nach dem Preisgesetz, eine "eine ganz geringe Zahl von Waren von außerordentlicher Bedeutung für die volkswirtschaftliche Entwicklung und das Leben der Bevölkerung", sondern die gesamten Schlüsselindustrien sollen vom Staatskapital kontrolliert werden. Das heißt nicht, daß sie nur aus staatseigenen Unternehmen bestehen sollen. Das Kapital nichtstaatlicher Minderheitsaktionäre und die Staatsindustrie ergänzende nichtstaatliche Unternehmen sind auch in den Schlüsselindustrien willkommen. Aber die Schlüsselindustrien sollen vom Staat, insbesondere der Staatsvermögenskommission zusammenfassend gelenkt werden. Angestrebt wird die Bildung von wenigen Dutzend Großkonzernen unter zentraler staatlicher Kontrolle. Markt und Wettbewerb sind in diesem Bereich nur noch ein mögliches Werkzeug unter vielen. Die zuständige Staatsbehörde ordnet ihren "Markt" nach Gutdünken. Das Ministerium für die Informationsindustrie beispielsweise hat die (gegenwärtig) sechs Staatsunternehmen, die Telekommunikationsnetze betreiben, mehrfach durch Befehl von oben umorganisiert, um "ausgeglichene Marktverhältnisse" auf dem "Markt" der Netzdienstleistungen zu schaffen.

Ganz so deutlich wird $\int 7$ des vorliegenden Gesetzes nicht, aber deutlich genug. Auch $\int 7$ teilt die Wirtschaft in zwei Bereiche. Die Schlüsselindustrien ("Lebensadern der Volkswirtschaft" und der Staatssicherheit, vom Recht legitimierte Monopole - nicht etwa nur "natürliche" Monopole, wie nach dem Preisgesetz) ordnet der Staat, der hier alles unternehmerische Handeln, einschließlich der Preise "überwachen, steuern und kontrollieren" soll. Das soll die Verbraucher schützen und den technischen Fortschritt fördern. Für diesen Bereich gilt also nur $\int 7$ des Gesetzes. Der Rest des Gesetzes gilt nur außerhalb der Schlüsselindustrien.

Dieser $\int 7$ kann das Gesetz völlig entwerten.

$\int 7$ ist freilich ein Fremdkörper in diesem Gesetz. Im Regierungsentwurf des Gesetzes, der im Juni 2006 dem Gesetzgeber vorgelegt wurde, fand er sich noch nicht. Er steht im Gegensatz zu den Vorschriften gegen den Mißbrauch von Verwaltungsmacht im 5. Kapitel des Gesetzes. Dies Kapitel ist zwar weitgehend aus 21.4.01/1 übernommen, also geltendes Recht, war aber bei der Vorbereitung des Gesetzes umstritten wie kein anderer Teil des Gesetzes und ist letztlich erst in der Diskussion der Entwürfe von der informierten Öffentlichkeit durchgesetzt worden. Vor allem steht $\int 7$ aber im Widerspruch zu den allgemeinen Grundsätzen des Gesetzes, insbesondere zu $\int 4$ : Ein "einheitliches, offenes, 
wettbewerbliches, geordnetes Marktwirtschaftssystem" kann man das Wirtschaftssystem nach $\int 7$ nicht nennen.

Liebhaber staatskontrollierter Schlüsselindustrien halten ihr Modell zwar für ein harmonisch "geordnetes Marktsystem". Sie wollen keine Zentralverwaltungswirtschaft alten Stils. In ihrem von oben dirigierten "Markt" ist die staatliche Wirtschaftsplanung weitgehend aufgegeben, sind kleine Staatsunternehmen "freigesetzt", Wirtschaftsverwaltung und Staatskonzerne getrennt, Privatunternehmen mit einbezogen worden. Doch diese Nutzung der Staatsgewalt durch mächtige Funktionäre von Superkonzernen, womöglich noch im Verbund mit privaten Unternehmen, führt auf den "geordneten Märkten" nicht zu Ruhe und Ordnung, sondern zu wilden politischen Machtkämpfen - die sechs Netzbetreiber z.B. zerschneiden sich gegenseitig die Leitungen -, schützt nicht die Interessen der Verbraucher, erlaubt aber den Gewinnern solcher Machtkämpfe, sich ungehemmt zu bereichern.

"Geordnete Märkte" führen daher wie in Rußland, so in China unvermeidlich zu Skandalen wie im Luneng-Fall (vgl. 5.12.06/1 Anm.1) oder bei den Investitionen des Sohnes eines ZK-Mitglieds in Australien. Dagegen hilft, will man nicht zur Zentralverwaltungswirtschaft zurückkehren, nur vollständiger Wettbewerb. Deshalb könnten die Skandale dazu führen, daß $\int 7$ doch noch durch Richtlinien, Verwaltungsverordnungen u.ä. entschärft wird.

Erst einmal muß man jedoch froh sein, daß die bisherigen Vorschriften, wie das Gesetz gegen unlauteren Wettbewerb (2.9.93/1), das Preisgesetz (29.12.97/1) und die Vorschriften gegen die Abschottung von Territorien (21.4.01/1), Preismonopole (18.6.03/1) und Dumping (3.8.99), noch weitergelten.

Das vorliegende Gesetz bringt gegenüber dem bisherigen Recht aber auch wichtige Fortschritte.

- Es faßt auf Gesetzesebene, nicht nur verstreut über Verordnungen, die Grundzüge des Antimonopolrechts zusammen, gibt ihnen damit erheblich größere Autorität, und erleichtert in diesem international stark vereinheitlichten Gebiet den hier sehr intensiv forschenden chinesischen Rechtsvergleichern den Hinweis auf ausländische Parallelen und damit auch die Fortentwicklung des chinesischen Antimonopolrechts.

- Es detailliert vieles, was bisher nur andeutungsweise geregelt war, so in $\iint 18$ und 19 die Definition der Marktbeherrschung.

- Bisher ist das Antimonopolrecht weitgehend auf den Warenverkehr beschränkt (vgl. insbesondere 21.4.01/1 \3 II !). Die Definition der "Waren" in der Klammer in $\int 12$ II des Gesetzes bezieht jetzt alle Dienstleistungen mit ein.

<2> Allgemeininteresse: wörtlich "öffentliches Interesse der Gesellschaft".

$<3>$ "Branchen, die...": gemeint ist: Branchen, deren Unternehmen...

$<4>$ Chin. jingyingzhe, eigentlich "Unternehmer" oder "Betreiber"; diesen Begriff hat das Gesetz aus dem Preisgesetz, 29.12.97/1, übernommen; hier wird er um 
des Einklangs mit deutschem und europäischem Wettbewerbsrecht willen als "Unternehmen" übersetzt.

$<5>$ "behindern, beeinflussen kann": gemeint wohl: negativ oder positiv beeinflussen kann.

$<6>$ Das ist rechnerisch nur im Fall der Nr.3 und nur bei einem der drei dann einzurechnenden Unternehmen möglich.

$<$ 7> Entspricht 21.4.01/1 Nr.3.

$<$ 8> Entspricht 21.4.01/1 Nr.4.

<9> Entspricht 21.4.01/1 Nr.5.

$<10>$ Entspricht 21.4.01/1 Nr.2.

$<11>$ Entspricht 21.4.01/1 Nr.6.

$<12>$ Entspricht 21.4.01/1 Nr.7.

$<13>$ Nach 29.4.99/1. 


\section{Literaturverzeichnis}

Altin-Sieber, Inci; Joint Ventures, Technologietransfer und -schutz, Heidelberg 1996. (zit.: Altin-Sieber)

An, Li; Guoji Jishu Zhuanrangfa(Das internationale Recht zum Technologietransfer), Aufl. 1, Beijing 2003. (zit.: An)

Ann, Christoph/Barona, Alfredo; Schuldrechtsmodernisierung und gewerblicher Rechtsschutz, Köln, Berlin, Bonn, München 2002. (zit.: Ann/Barona)

Aracama-Zorraquin, Ernesto; Gegenwärtiger Zustand und Entwicklungstendenzen des Patentrechts in Lateinamerika, GRURInt. 1972 Heft 05. S. 139 ff. (zit.: Aracama-Zorraquin, GRURInt. 1972)

Aracama-Zorraquin, Ernesto; Der gewerbliche Rechtsschutz in Lateinamerika Beginn einer neuen Entwicklung, GRURInt. 1976 Heft 02. S. 53 ff. (zit.: Aracama-Zorraquin, GRURInt. 1976)

Axster, Herbert; Die Lizenz als Rationalisierungsmittel, GRUR 1964 Heft 05. S. $229 \mathrm{ff}$.

Bartling, Dieter; Die Rolle der Patente bei der Übertragung von Technologie auf Entwicklungsländer aus der Sicht der pharmazeutischen Industrie, GRURInt. 1972 Heft 11. S. 396 ff. (zit.: Bartling, GRURInt. 1972)

Bechert, Rudolf; Der Lizenzvertrag, Rosenheim 1949. (zit.: Bechert)

Beier, Friedrich-Karl; Die herkömmlichen Patentrechtstheorien und die sozialistische Konzeption des Erfinderrechts, GRURInt. 1970 Heft 01. S. 1 ff. (zit.: Beier, GRURInt. 1970)

Beier, Friedrich-Karl/Kunz, Hans Pater; Die Bedeutung des Patentrechts für den Transfer von Technologie in Entwicklungsländer, GRURInt. $1972 \mathrm{Heft}$ 11. S. 385 ff. (zit.: Beier/Kunz, GRURInt. 1972)

Beier, Friedrich-Karl; Die Bedeutung des Patentsystems für den technischen, wirtschaftlichen und sozialen Fortschritt, GRURInt. 1979 Heft 05. S. 227 ff. (zit.: Beier, GRURInt. 1979)

Beier, Friedrich-Karl; Hundert Jahre Pariser Verbandsübereinkunft - Ihre Rolle in Vergangenheit, Gegenwart und Zukunft, GRURInt. 1983 Heft 6-7. S. 339 ff. (zit.: Beier, GRURInt. 1983)

Beier, Friedrich-Karl; Gewerblicher Rechtsschutz und freier Warenverkehr im europäischen Binnenmarkt und im Verkehr mit Drittstaaten, GRURInt. 1989 Heft 8-9. S. 603 ff. (zit.: Beier, GRURInt. 1989)

Beier, Friedrich-Karl; Zur Zulässigkeit von Parallelimporten patentierter Erzeugnisse, GRURInt. 1996 Heft 01. S. 1 ff. (zit.: Beier, GRURInt. 1996)

Beier, Friedrich-Karl; Patent- und Musterrecht, Aufl. 7, München 2004. (zit.: Beier) 
Benkard, Georg; Patentgesetz, Gebrauchsmustergesetz, Aufl. 10, München 2006.(zit.: Benkard)

Berlit, Wolfgang; Markenrecht, Aufl. 6, München 2005. (zit.: Berlit, Markenrecht)

Blasek, Katrin; Erschöpfung und Paralleleinfuhr in der VR China - Rechtslage und Ausblick, GRURInt. 2006 Heft 7. S. 568 ff. (zit.: Blasek, GRURInt. 2006)

Blum, Rudolf E./Pedrazzini, Mario M.; Das schweizerische Patentrecht, Band I/II/III, Bern 1961. (zit.: Blum/Pedrazzini)

Böckstiegel, Karl-Heinz/Krämer, Paul Michael/Polley, Isabel; Kann der Betrieb von Satelliten im Weltraum patentrechtlich geschützt werden? GRUR 1999 Heft 01. S. 1 ff. (zit.: Böckstiegel, GRUR 1999)

Boguslawskij, Marc M.; Internationaler Technologietransfer, Heidelberg 1990. (zit.: Boguslawskij)

Böhme, Werner; Die Besteuerung des Know-how; München, Berlin 1967. (zit.: Böhme)

Bohnet, Michael; Das Nord-Süd-Problem: Konflikte zwischen Industrie- und Entwicklungsländern, Aufl. 3, München 1974. (zit.: Bohnet, Das Nord-Süd-Problem)

Bohnet, Uwe; Das Markenrecht in der Volksrepublik China und Russland, Köln, Berlin, Bonn, München 1995. (zit.: Bohnet)

Bottenschein, Florian; Die Bekämpfung der Markenpiraterie in der Volksrepublik China und Hongkong, GRURInt. 2005 Heft 2. S. 121 ff. (zit.: Bottenschein, GRURInt. 2005)

Brandi-Dohrn, Matthias; Sukzessionsschutz bei der Veräußerung von Schutzrechten, GRUR 1983 Heft 04. S. 146 ff. (zit.: Brandi-Dohrn, GRUR 1983)

Brandi-Dohrn, Matthias/Gruber, Stephan/Muir, Ian; Europäisches und internationales Patentrecht, München $2002 . \quad$ (zit.: Brandi-Dohrn/Gruber/Muir)

Bühring, Manfred; Gebrauchsmustergesetz, Kommentar, Aufl. 6, Köln 2002. (zit.: Bühring)

Busse, Rudolf/Woesler, Emmi; Warenzeichengesetz nebst Pariser Verbandsübereinkunft und Madrider Abkommen, Kommentar, Aufl. 5, Berlin 1976. (zit.: Busse, Warenzeichengesetz)

Busse, Rudolf; Patentgesetz unter Berücksichtigung des Europäischen Patentübereinkommens und des Patentzusammenarbeitsvertrags, Kommentar, Aufl. 6, Berlin 2003. (zit.: Busse, Patentgesetz)

Byrne, Noel; Licensing Technology, Aufl. 2, Bristol 1998. (zit.: Byrne)

Chrocziel, Peter; Einführung in den Gewerblichen Rechtsschutz und das Urheberrecht, Aufl. 2, München 2002 (zit.: Chrocziel)

Correa, Carlos M.; Veränderungen im Lateinamerikanischen Patentrecht, GRURInt. 1994 Heft 10. S. 799 ff. (zit.: Correa, GRURInt. 1994)

Davies, Emma/Yow, Chengli; China's Anti-trust Regime, China Law \& Practice, 29. Mai 2005. (zit.: Davies/Yow) 
Delp, Ludwig; Das Recht des geistigen Schaffens in der Informationsgesellschaft, Aufl. 2, München 2003. (zit.: Delp)

Dessemontet, François; The legal protection of know-how in the United States of America, Aufl. 2, Geneva 1976. (zit.: Dessemontet)

Dietz, Adolf(Hrsg.); Die Neuregelung des gewerblichen Rechtsschutzes in China, Weinheim 1988. (zit.: Dietz)

Dreier, Thomas; TRIPS und die Durchsetzung von Rechten des geistigen Eigentums, GRURInt. 1996 Heft 03. S. 205 ff. (zit.: Dreier, GRURInt. 1996)

Dreier, Thomas/Schulze, Gernot; UrhG-Kommentar, München 2004. (zit.: Dreier/Schulze)

Drexl, Josef; Die neue Gruppenfreistellungsverordnung über Technologietransfer-Vereinbarungen im Spannungsfeld von Ökonomisierung und Rechtssicherheit, GRURInt. 2004 Heft 9. S. 716 ff. (zit.: Drexl, GRURInt. 2004)

Dreyer, Gunda/Kotthoff, Jost/Meckel, Astrid; Heidelberger Kommentar zum Urheberrecht, Heidelberg 2004. (zit.: Dreyer/Kotthoff/Meckel)

Duscha, Waldemar; Technologietransfer in die Volksrepublik China durch Wirtschaftskooperation, Hamburg 1987. (zit.: Duscha)

Eichmann, Helmut; Geschmacksmustergesetz, Aufl. 3, München 2005. (zit.: Eichmann)

Ekey, Friedrich L./Klippel, Diethelm/Kotthoff, Jost/Meckel, Astrid/Plaß, Gunda; Heidelberger Kommentar zum Wettbewerbsrecht, Aufl. 2, Heidelberg 2004. (zit.: Ekey/Klippel)

Emmerich, Volker; Kartellrecht, Aufl. 9, München 2001. (zit.: Emmerich)

Emmerich, Volker; Unlauterer Wettbewerb, Aufl. 7, München 2004. (zit.: Emmerich, Unlauterer wettbewerb)

Ensthaler, Jürgen; Gewerblicher Rechtsschutz und Urheberrecht, Aufl. 2, Heidelberg 2003. (zit.: Ensthaler)

Faupel, Rainer; GATT und geistiges Eigentum - Ein Zwischenbericht zu Beginn der entscheidenden Verhandlungsrunde, GRURInt. 1990 Heft 04. S. 255 ff. (zit.: Faupel, GRURInt. 1990)

Fezer, Karl-Heinz; Markenrecht, Kommentar zum Markengesetz, zur Pariser Verbandsübereinkunft und zum Madrider Markenabkommen, Dokumentation des nationalen, europäischen und internationalen Kennzeichenrechts, Aufl. 3, München 2001. (zit.: Fezer)

Fezer, Karl-Heinz; Lauterkeitsrecht, Kommentar zum Gesetz gegen den unlauteren Wettbewerb (UWG), Band 2: \$S 5-22 UWG, München 2005. (zit.: Fezer, UWG)

Fikentscher, Wolfgang/Kunz-Hallstein, Hans Peter; Technologietransfer an Entwicklungsländer und Kartellrecht, GRURInt. 1979 Heft 10. S. 437 ff. (zit.: Fikentscher/Kunz-Hallstein, GRURInt. 1979)

Fikentscher, Wolfgang/Immenga, Ulrich; Draft International Antitrust Code, Aufl. 1, Baden-Baden 1995. (Fikentscher, Draft International Antitrust Code) 
Fikentscher, Wolfgang; Wettbewerbsrecht im TRIPS-Agreement der Welthandelsorganisation - Historische Anknüpfung und Entwicklungschancen, GRURInt. 1995 Heft 07. S. 529 ff.

Fikentscher, Wolfgang; The Draft International Antitrust Code: Objections and Rejoinders, IIC Vol. 26 No. 6/1995. S. 999 ff. (zit.: Fikentscher, IIC 1995)

Fikentscher, Wolfgang; An International Antitrust Code, IIC 1996 Heft 06. S. 755 ff. (zit.: Fikentscher, IIC 1996)

Finger, Peter; Die Offenkundigkeit des mitgeteilten Fachwissens bei Know-how-Verträgen, GRUR 1970 Heft 01. S. 3 ff.

Fischer, Ernst; Das Know-how, GRURInt. 1970 Heft 07. S. 240 ff. (zit.: Fischer, GRURInt. 1970)

Fischer, Friedrich B.; Grundzüge des Gewerblichen Rechtsschutzes, Aufl. 2, Köln, Berlin, Bonn, München 1986. (zit.: Fischer)

Forkel, Hans; Zur Übertragbarkeit geheimer Kenntnisse, in: Festschrift für Ludwig Schnorr von Carolsfeld zum 70. Geburtstag 26. Januar 1973, Herausgegeben von Heinrich Hubmann und Heinz Hübner, Köln, Berlin, Bonn, München 1973, S. 105 ff. (zit.: Forkel, FS Schnorr v. Carolsfeld)

Forkel, Hans; Gebundene Rechtsübertragungen, Köln, Berlin, Bonn, München 1977. (zit.: Forkel)

Forkel, Hans; Zur dinglichen Wirkung einfacher Lizenzen, NJW 1983 Heft 32. S. 1764 ff. (zit.: Forkel, NJW 1983)

Forkel, Hans/Kraft, Alfons; Beiträge zum Schutz der Persönlichkeit und ihrer schöpferischen Leistungen, Festschrift für Heinrich Hubmann zum 70. Geburtstag, Frankfurt am Main 1985

Fromm, Friedrich Karl/Nordemann, Wilhelm; Urheberrecht, Kommentar zum Urheberrechtsgesetz und zum Urheberrechtswahrnehmungsgesetz, Aufl. 9, Stuttgart 1998. (zit.: Fromm/Nordemann)

Funke, Guido; Technologietransfer in internationalen Corporate Joint Ventures, Frankfurt am Main 1998. (zit.: Funke)

v. Gamm, Otto-Friedrich Frhr.; Urheberrechtsgesetz, Kommentar, München 1968. (zit.: v. Gamm)

Ganea, Peter; Die Neuregelung des chinesischen Patentrechts, GRURInt. 2002 Heft 8-9. S. 686 ff. (zit.: Ganea, GRURInt. 2002)

Ganea, Peter; Volksrepublik China - Neue Zollbestimmungen zum Schutz des geistigen Eigentums, GRURInt. 2004 Heft 6. S. 532 f. (zit.: Ganea, GRURInt. 2004)

Ganea, Peter; Volksrepublik China - Änderung des Außenhandelsgesetzes, GRURInt. 2004 Heft 10. S. 889. (zit.: Ganea, GRURInt. 2004)

Ganea, Peter/Pattloch, Thomas; Intellectual Property Law in China, Den Haag 2005. (zit.: Ganea/Pattloch)

Gaster, Jens; Die Erschöpfungsproblematik aus der Sicht des Gemeinschaftsrechts, GRURInt. 2000 Heft 07. S. 571 ff. (zit.: Gaster, GRURInt. 2000) 
Gaul, Dieter/Bartenbach, Kurt/Gennen, Klaus; Patentlizenz- und Know-how-Vertrag, Aufl. 4, Köln 1997(zit.: Gaul/Bartenbach)

Geissler, Bernhard/Pagenberg, Jochen; Der Software-Lizenzvertrag in der Praxis, in: Lehmann, Michael, Rechtsschutz und Verwertung von Computerprogrammen, S. 629 ff. (zit.: Geissler/Pagenberg)

Gericke; Die Wirkung der Zurechnungsvorschrift des $\int 8$ Ziff. 7 GewStG bei Lizenzen, Know-how-Verträgen und gemischten Verträgenm Finanzrundschau 1963, S. 330 ff.

Gitter, Wolfgang; Gebrauchsüberlassungsverträge, Tübingen 1988(zit.: Gitter)

Glatter, Joachim; Rechtsgrundlagen für Handel und wirtschaftliche Kooperation in der Volksrepublik China, Hamburg 1989

Götting, Horst-Peter; Der Begriff des Geistigen Eigentums, GRUR 2006 Heft 5. S. $353 \mathrm{ff}$.

Groß, Friedrich; Wie entsteht eine herrschende Meinung? - (Dargestellt an der angeblichen Ausführungspflicht des Lizenznehmers), GRUR $1951 \mathrm{Heft}$ 8-9. S. 369 ff. (zit.: Groß, GRUR 1951)

Guilino, Ernst; Zur Frage der Notwendigkeit ausschließlicher Lizenzen, GRUR 1974 Heft 04. S. 187 ff. (zit.: Guilino)

Guo, Shoukang; Entstehung und Grundzüge des chinesischen Patentgesetzes, GRURInt. 1985 Heft 01. S. 1 ff. (zit.: Guo, GRURInt. 1985)

Guo, Shoukang; Drafting and Promulgation of the Chinese Patent Law, IIC Vol. 16 No. 4/1985. S. 367 ff. (zit.: Guo, IIC 1985)

Guo, Shoukang; Entwicklung und Perspektiven des geistigen Eigentums in der Volksrepublik China, GRURInt. 1997 Heft 12. S. 949 ff. (zit.: Guo, GRURInt. 1997)

Guo, Shoukang; Guoji Jishu Zhuanrang(Der internationale Technologietransfer), Aufl. 9, Beijing 1998

Guojia Zhishichanquanju Tiaofasi (Vorschriften- und Gesetzesabteilung des Staatsamtes für geistiges Eigentum), Xinzhuanlifa Xiangjie (Kommentar zum neuen Patentgesetz), Beijing 2001. (zit.: PatG-Kommentar)

Haedicke, Maximilian; Die Gewährleistungshaftung bei Patentveräußerungs- und Patentlizenzverträgen und das neue Schuldrecht, GRUR 2004 Heft 02. S. 123 ff. (zit.: Haedicke GRUR 2004)

Hansen, Barbara; Economic Aspects of Technology Transfer to Developing Countries, IIC Vol. 11 No. 4/1980. S. 429 ff. (zit.: Hansen, IIC Vol. 11 No. 4/1980.)

Harte-Bavendamm, Henning/Henning-Bodewig, Frauke; Gesetz gegen den unlauteren Wettbewerb (UWG)-Kommentar, München 2004. (zit.: Harte-Bavendamm/Henning-Bodewig)

Hasselblatt, Gordian N.; Münchener Anwalts Handbuch, Gewerblicher Rechtsschutz, München 2001. (zit.: Hasselblatt)

Haug, David M.; The International Transfer of Technology: Lessons that east europe can learn from the failed third world experience, Harvard Journal, Volume 5, Spring Issue, 1992. 
Haver, Friedrich/Mailänder, Karl Peter; Lizenzvergabe durch deutsche Unternehmen in das Ausland, Heidelberg 1967. (zit.: Haver/Mailänder)

Heath, Christopher; Bedeutet TRIPS wirklich eine Schlechterstellung von Entwicklungsländern, GRURInt. 1996 Heft 12. S. 1169 ff. (zit.: Heath, GRURInt. 1996)

Hefermehl, Wolfgang/Köhler, Helmut/Bornkamm, Joachim; Wettbewerbsrecht, Aufl. 24, München 2006. (zit.: Hefermehl/Köhler/Bornkamm)

Henn, Günter; Problematik und Systematik des internationalen Patentlizenzvertrages, München, Berlin 1967. (zit.: Henn, Problematik)

Henn, Günter; Patent- und Know-how-Lizenzvertrag, Aufl. 4, Heidelberg 1999. (zit.: Henn)

Herdegen, Matthias; Internationales Wirtschaftsrecht Aufl. 4, München 2003. (zit.: Herdegen)

Hesse, Hans Gerd; Die kartellrechtliche Behandlung von Verträgen über nicht geheimes technisches Know-how, GRUR 1985 Heft 08. S. 661 ff. (zit.: Hesse, GRUR 1985)

Heuser, Robert/Zhao, Hang; Technologietransferrecht der VR China, RIW 1985 Heft 9. S. 684 ff. (zit.: Heuser/Zhao, RIW 1985)

Heuser, Robert(Hrsg.); Das Außenwirtschaftsrecht der Volksrepublik China, München 1986.

Heuser, Robert; Ausführungsregeln zum chinesischen Recht der Technikimporte, RIW 1988 Heft 4. S. 258 ff. (zit.: Heuser, RIW 1988)

Heuser, Robert(Hrsg.); Wirtschaftsreform und Gesetzgebung in der VR China, Hamburg 1996.

Heuser, Robert/Klein, Roland; Die WTO und das neue Ausländerinvestitionsund Außenhandelsrecht der VR China, Gesetze und Analysen, Hamburg 2004

Heuser, Robert(Hrsg.); Beiträge zum chinesischen Zivil- und Wirtschaftsrecht, Hamburg 2005

Hiance, N./Plasseraud, Y.; Brevets et sous-développement, Paris 1972. (zit.: Hiance/Plasseraud)

Hilf, Meinhard/Göttsche, Götz J.; Chinas Beitritt zur WTO, RIW 2003 S. 161 ff.

Hillig, Hans-Peter; Urheber- und Verlagsrecht, Aufl. 10, München 2003. (zit.: Hillig)

Hilpert, Hanns Günther; TRIPS und das Interesse der Entwicklungsländer am Schutz von Immaterialgüterrechten in ökonomischer Sicht, GRURInt. 1998 Heft 02. S. 91 ff. (zit.: Hilpert, GRURInt. 1998)

Holyoak, Jon/Torremans, Paul; Intellectual Property Law, London, Dublin, Edinburgh 1995. (zit.: Holyoak/Torremans)

Hoppe, Jürgen F.; Lizenz- und Know-how-Verträge im internationalen Privatrecht, Frankfurt am Main 1994(zit.: Hoppe)

Huber, Bertram; Technologietransfer-, Know-how- und Lizenzverträge mit Partnern in der Volksrepublik China, Backnang 1986. (zit.: Huber)

Hubmann, Heinrich/Götting, Horst-Peter; Gewerblicher Rechtsschutz, Aufl. 7, 
München 2002. (zit.: Hubmann/Götting)

Huck, Winfried; Rahmenbedingungen für den Technologietransfer von Deutschland nach China, Institute for International Business \& Law, www.law-and-business.com, 12. Januar 2005. (zit.: Huck)

Hüfner, Klaus/Naumann, Jens; Neue Weltwirtschaftsordnung?: der Nord-Süd-Konflikt im UNO-System, Aufl. 2, Berlin 1980.

Imam, Ali M.; How Does Patent Protection Help Developing Countries? IIC 2006 Heft 3. S. 245 ff. (zit.: Imam, IIC 2006)

Ingerl, Reinhard/Rohnke, Christian; Markengesetz, Gesetz über den Schutz von Marken und sonstigen Kennzeichen, Kommentar, Aufl. 2, München 2003. (zit.: Ingerl/Rohnke)

Isay, Hermann; Patentgesetz und Gesetz, betreffend den Schutz von Gebrauchsmustern, Berlin 1932. (zit.: Isay)

Julius, Hinrich/Müller, Matthias; Das neue chinesische Außenhandelsrecht, ZChinR 2004 S. 215 ff. (zit.: Julius/Müller, ZChinR 2004)

Klauer, Georg/Möhring, Philipp; Patentrechtskommentar, Band I, Aufl. 3, München 1971. (zit.: Klauer/Möhring)

Knap, Karel; Der Lizenzvertrag als ein besonderer Vertragstypus, GRURInt. 1973 Heft 6-7. S. 226 ff. (zit.: Knap, GRURInt. 1973)

Knoppe , Helmut; Die Besteuerung der Lizenz- und Know-how-Verträge, Aufl. 2, Köln 1972. (zit.: Knoppe)

Kohler, Joseph; Handbuch des deutschen Patentrechts in rechtsvergleichender Darstellung, Mannheim 1900. (zit.: Kohler)

Köhler, Helmut; Wettbewerbsrecht und Kartellrecht, Aufl. 26, München 2005. (zit.: Köhler)

Körner, Eberhard; Der Bestand bzw. Fortbestand von Schutzrechten und Know-how als Voraussetzung der Lizenzgebühren - bzw. Schadensersatzpflicht, GRUR 1982 Heft 06. S. 341 ff. (zit.: Körner, GRUR 1982)

Kortunay, Ayhan; Patentlizenz- und Know-How-Verträge im deutschen und europäischen Kartellrecht, Köln 2003. (zit.: Kortunay)

Krasser, Rudolf; Der Schutz des Know-how nach deutschem Recht, GRUR 1970 Heft 12. S. 587 ff. (zit.: Krasser, GRUR 1970)

Krasser, Rudolf; Grundlagen des zivilrechtlichen Schutzes von Geschäfts- und Betriebsgeheimnissen sowie von Know-how, GRUR 1977 Heft 04. S. 177 ff. (zit.: Krasser, GRUR 1977)

Krasser, Rudolf/Schmid, Hans Dieter; Der Lizenzvertrag über technische Schutzrechte aus der Sicht des deutschen Zivilrechts, GRURInt. 1982 Heft 05. S. 324 ff. (zit.: Krasser/Schmid, GRURInt. 1982)

Krasser, Rudolf; Patentrecht, Aufl. 5, München 2004. (zit.: Krasser)

Kunz-Hallstein, Hans Peter; Patentschutz, Technologietransfer und Entwicklungsländer eine Bestandsaufnahme, GRURInt. 1975 Heft 8-9. S. 261 ff. (zit.: Kunz-Hallstein, GRURInt. 1975) 
Langen, Eugen; Internationale Lizenzverträge, Weinheim 1954

Langhammer, Rolf Johannes/Stecher, Bernd; Der Nord-Süd-Konflikt: die Spielregeln der Weltwirtschaft im Brennpunkt, Würzburg 1980.

Lauffs, Andreas; Guide to China and the WTO, Baker \& McKenzie, Hong Kong 2002. (zit.: Lauffs)

Lehmann, Michael; Eigentum, geistiges Eigentum, gewerbliche Schutzrechte Property Rights als Wettbewerbsbeschränkungen zur Förderung des Wettbewerbs, GRURInt. 1983 Heft 6-7. S. 356 ff.

Lettl, Tobias; Kartellrecht, München 2005. (zit.: Lettl)

Lindenmaier, Fritz/Weiss, Ulrich; Das Patentgesetz, Aufl. 6, Köln, Berlin, Bonn, München 1973. (zit.: Lindenmaier/Weiss)

Link, Werner; Der Nord-Süd-Konflikt und die Zusammenarbeit der Entwicklungsländer, Berlin 1985.

Liu, Xiaohai; Durchsetzung von Rechten des geistigen Eigentums in der Volksrepublik China, GRURInt. 1999 Heft 6. S. 488 ff. (zit.: Liu, GRURInt. 1999)

Loewenheim, Ulrich; Gewerbliche Schutzrechte, freier Warenverkehr und Lizenzverträge, GRUR 1982 Heft 08. S. 461 ff. (zit.: Loewenheim, GRUR 1982)

Loewenheim, Ulrich; Nationale und internationale Erschöpfung von Schutzrechten im Wandel der Zeiten, GRURInt. 1996 Heft 04. S. 307 ff. (zit.: Loewenheim, GRURInt. 1996)

Loewenheim, Ulrich; Handbuch des Urheberrechts, München 2003. (zit.: Loewenheim)

Lorenz, Moritz; Die bevorstehende chinesische Kartellgesetzgebung, GRURInt. 2006 Heft 6. S. 468 ff. (zit.: Lorenz, GRURInt. 2006)

Loth, Hans-Friedrich; Gebrauchsmustergsetz, München 2001. (zit.: Loth)

Lübbig, Thomas; „... et dona ferentes”: Anmerkungen zur neuen EG-Gruppenfreistellungsverordnung im Bereich des Technologietransfers, GRUR 2004 Heft 6. S. 483 ff. (zit.: Lübbig, GRUR 2004)

Lüdecke, Wolfgang; Die Ausübungspflicht des Lizenznehmers, GRUR $1952 \mathrm{Heft}$ 5-6. S. 211 ff. (zit.: Lüdecke, GRUR 1952)

Lüdecke, Wolfgang/Fischer, Ernst; Lizenzverträge, Weinheim 1957(zit.: Lüdecke/Fischer)

Lundstedt, Lydia; Gerichtliche Zuständigkeit und Territorialitätsprinzip im Immaterialgüterrecht - Geht der Pendelschlag zu weit? GRURInt. 2001 Heft 2. S. 103 ff. (zit.: Lundstedt, GRURInt. 2001)

Machlup, Fritz; An Economic Review of the Patent System, study No. 50 of the Subcommittee on Patents, Trademarks, and Copyright of the Committee on the Judiciary - United States Senate, $85^{\text {th }}$ Congress, 2d Session, Washington 1958; leicht gekürzte deutsche Übersetzung: Die wirtschaftlichen Grundlagen des Patentrechts, GRURAusl. $1961 \mathrm{Heft}$ 8-9. (zit.: Machlup, GRURAusl. 1961) 
Mager, Heinrich; Einfache Lizenz bei Veräußerung des Schutzrechts, GRUR 1983 Heft 02. S. 51 ff. (zit.: Mager, GRUR 1983)

Mager, Knut; Zur Zulässigkeit von Parallelimporten patentgeschützter Waren, GRUR 1999 Heft 8-9. S. 637 ff. (zit.: Mager, GRUR 1999)

Mailänder, Peter; Vereinbarungen zur Know-how-Überlassung im Wettbewerbsrecht der EWG, GRURInt. 1987 Heft 8-9. S. 523 ff. (zit.: Mailänder, GRURInt. 1987)

Mangalo, Nasrallah; Patentschutz und Technologietransfer im Nord-Süd-Konflikt, GRURInt. 1977 Heft 10. S. 349 ff. (zit.: Mangalo, GRURInt. 1977)

Mansani, Luigi; Gesetz vom 7. April 2000 über Urheberrecht, verwandte Schutzrechte und die Änderung einiger Gesetze(Urhebergesetz), GRURInt. 2000 Heft 10. S. 896 ff. (zit.: Mansani, GRURInt. 2000)

Meili, Tobias; Der Schutz von Know-how nach schweizerischem und internationalem Recht - Anpassungsbedarf aufgrund des TRIPS-Abkommens? Bern 2000. (zit.: Meili)

Mes, Peter; Patentgesetz, Gebrauchsmustergesetz, Kommentar, Aufl. 2, München 2005. (zit.: Mes)

Meyer, Andreas; Die EG-Gruppenfreistellungsverordnung zum Technologietransfer, GRURInt. 1997 Heft 06. S. 498 ff. (zit.: Meyer, GRURInt. 1997)

Möhring/Nicolini, Käte; Urheberrechtsgesetz, Kommentar, Aufl. 2, München 2000. (zit.: Möhring/Nicolini)

Münzel, Frank; Unternehmens- und Gesellschaftsrecht der VR China, Hamburg 1989

Nettesheim, Martin/Sander Gerald G.; WTO-Recht und Globalisierung, Berlin 2003. (zit.: Nettesheim/Sander)

Nirk, Rudolf; Die Einordnung der Gewährleistungsansprüche und Leistungsstörungen bei Verträgen über Patente in das Bürgerliche Gesetzbuch, GRUR 1970 Heft 07. S. 329 ff.(zit.: Nirk, GRUR 1970)

Nirk, Rudolf/Kurtze, Helmut; Geschmacksmustergesetz, Aufl. 2, Köln, Berlin, Bonn, München 1997. (zit.: Nirk/Kurtze)

Nordemann, Wilhelm/Nordemann, Axel/Nordemann, Jan Bernd; Wettbewerbsund Markenrecht, Aufl. 10, Baden-Baden 2004. (zit.: Nordemann)

Oppenländer, Karl Heinrich; Die Wirkungen des Patentwesens im Innovationsprozess, GRUR 1977 Heft 06. S. 362 ff. (zit.: Oppenländer, GRUR 1977)

Oppenländer, Karl Heinrich; Die wirtschaftspolitische Bedeutung des Patentwesens aus der Sicht der empirischen Wirtschaftsforschung, GRURInt. 1982 Heft 10. S. 598 ff. (zit.: Oppenländer, GRURInt. 1982)

Oppenländer, Karl Heinrich; Patentwesen, technischer Fortschritt und Wettbewerb, Berlin-München 1984. (zit.: Oppenländer)

Osterrieth, Christian; Die Neuordnung des Rechts des internationalen Technologietransfers, Der UNCTAD Code of Conduct on Transfer of Technology und die Revision der Pariser Verbandsübereinkunft zum 
Schutze des gewerblichen Eigentums, Konstanz 1986. (zit.: Osterrieth)

Osterrieth, Christian; Patentrecht, Aufl. 2, München 2004.(zit.: Osterrieth, Patentrecht)

Pacón, Ana Maria; Aktuelle Tendenzen im gewerblichen Rechtsschutz Lateinamerikas: Verstärkung und Harmonisierung des Schutzes, GRURInt. 1994 Heft 11. S. 888 ff. (zit.: Pacón, GRURInt. 1994)

Pacón, Ana Maria; Was bringt TRIPS den Entwicklungsländern? GRURInt. 1995 Heft 11. S. 875 ff. (zit.: Pacón, GRURInt. 1995)

Pagenberg, Jochen/Geissler, Bernhard; Lizenzverträge, License Agreements, Aufl. 5, Köln, Berlin, Bonn, München 2003. (zit.: Pagenberg/Geissler)

Pagenberg, Jochen; Lizenzverträge, München 1985. (zit.: Pagenberg, Lizenzverträge)

Palandt, Otto; Bürgerliches Gesetzbuch, Aufl. 65, München 2006. (zit.: Palandt)

Pattloch, Thomas; Die Neuordnung des internationalen Technologietransfers in der VR China, GRURInt. 2003 Heft 8-9. S. 695 ff. (zit.: Pattloch, GRURInt. 2003)

Pedrazzini, Mario M.; Patent- und Lizenzvertragsrecht, Aufl. 2, Bern 1987. (zit.: Pedrazzini, Patentrecht)

Pedrazzini, Mario M.; Immaterialgüter- und Wettbewerbsrecht, Bern 1998. (zit.: Pedrazzini)

Peetz, Wolfgang-Martin; Die Nichtangriffsklausel in Lizenzverträgen, Göttingen 1961. (zit.: Peetz)

Peng, Wanlin (Hrsg.); Mingfa Xue (Studien zum Zivilrecht), Aufl. 5, Beijing 1995. (zit.: Peng, Wanlin)

Pfaff, Dieter; Der Know-how-Vertrag im bürgerlichen Recht, Dogmatische Einordnung, Haftungsvoraussetzungen und Haftungsfolgen, BB 1974, S. 565 ff. (zit.: Pfaff, BB 1974)

Pfaff, Dieter/Nagel, Sibilla; Internationale Rechtsgrundlagen für Lizenzverträge im gewerblichen Rechtsschutz, International Sources for Licensing of Industrial Property, München 1993.

Pfaff, Dieter/Osterrieth, Christian; Lizenzverträge, Formularkommentar, Aufl. 2, München 2004. (zit.: Pfaff/Osterrieth)

Pfanner, Klaus; Förderung der technischen Entwicklung und gewerblicher Rechtsschutz, GRURInt. 1983 Heft 6-7. S. 362 ff. (zit.: Pfanner, GRURInt. 1983)

Pfister, Bernhardt; Das technische Geheimnis „Know how“ als Vermögensrecht, München 1974. (zit.: Pfister)

Prieß, Hans-Joachim/Berrisch, Georg M.; WTO-Handbuch, München 2003

Rasch, Harold; Der Lizenzvertrag in rechtsvergleichender Darstellung, Berlin 1933. (zit.: Rasch)

Rebmann, Kurt/Säcker, Franz Jürgen (Hrsg.); Münchener Kommentar zum Bürgerlichen Gesetzbuch, Band 2a Schuldrecht Allgemeiner Teil SS 241-432, Aufl. 4, München 2003. (zit.: MünchKomm/Bearb.)

Rehbinder, Manfred; Urheberrecht, Aufl. 14, München 2006. (zit.: Rehbinder) 
Rehmann, Thorsten; Geschmacksmusterrecht, München 2004. (zit.: Rehmann)

Reimer, Eduard; Patentgesetz und Gebrauchsmustergesetz, Systematischer Kommentar, Aufl. 3, Köln, Berlin, Bonn, München 1968. (zit.: Reimer)

Reinelt; Zur neueren Entwicklung des Patentschutzes für Arzneimittel in Lateinamerika, GRURInt. 1972 Heft 01. S. 12 ff. (zit.: Reinelt, GRURInt. 1972)

Riemer, Hans M.; Die beschränkten dinglichen Rechte, Grundriss des schweizerischen Sachenrechts, Band II, Bern 1986. (zit.: Riemer)

Rigamonti, Cyrill P.; Geistiges Eigentum als Begriff und Theorie des Urheberrechts, Baden-Baden 2001

Rosenberger, Hans-Peter; Nochmals, Zur Frage des Fortbestands der einfachen Lizenz bei Übertragung des Patents, GRUR 1983 Heft 05. S. 203 ff. (zit.: Rosenberger, GRUR 1983)

Sack, Rolf; Die Erschöpfung von gewerblichen Schutzrechten und Urheberrechten nach europäischem Recht, GRUR 1999 Heft 03. S. 193 ff. (zit.: Sack, GRUR 1999)

Sack, Rolf; Der Erschöpfungsgrundsatz im deutschen Immaterialgüterrecht, GRURInt. 2000 Heft 07. S. 610 ff. (zit.: Sack, GRURInt. 2000)

Schack, Haimo; Urheber- und Urhebervertragsrecht, Aufl. 3 Tübingen 2005. (zit.: Schack)

Schade, Peter; Die Ausübungspflicht bei Lizenzen, dargestellt an Verträgen über Patent-, Gebrauchsmuster- und Know-how-Lizenzen, Köln, Berlin, Bonn, München 1967. (zit.: Schade)

Schatz, Ulrich; Die Erschöpfung des Patentrechts - im Recht der Mitgliedstaaten der Europäischen Wirtschaftsgemeinschaft und nach dem Vorentwurf eines Übereinkommens über ein europäisches Patent für den Gemeinsamen Markt, GRURInt. 1970 Heft 07. S. 207 ff. (zit.: Schatz, GRURInt. 1970)

Scheil, Susanne/Scheil, Jörg-Michael; Praxis und Probleme der Anmeldung gewerblicher Schutzrechte in der Volksrepublik China, GRURInt. 1996 Heft 10. S. 999 ff. (zit.: Scheil, GRURInt. 1996)

Schmidt-Diemitz, Rolf; Geistiges Eigentum und entwicklungspolitischer Wissenstransfer, GRURInt. 1988 Heft. 04, S. 287 ff. (zit.: Schmidt-Diemitz, GRURInt. 1988)

Schricker, Gerhard; Urheberrecht, Kommentar, Aufl. 3, München 2006. (zit.: Schricker)

v. Schultz, Detlef; Kommentar zum Markenrecht, Heidelberg 2002. (zit.: v. Schultz)

Schumacher, Volker/Schmid, Christoph; Die neue Gruppenfreistellungsverordnung für TechnologietransferVereinbarungen, GRUR 2006 Heft 1. S. 1 ff. (zit.: Schumacher/Schmid, GRUR 2006)

Sehirali, Feyzan Hayal; Schutz des Know-how nach türkischem, deutschem und europäischem Recht, Köln 2004. (zit.: Sehirali) 
Shan, Xiaoguang; Technologietransferverträge im neuen chinesischen Vertragsgesetz, GRURInt. 2000 Heft 06. S. 506 ff. (zit.: Shan, GRURInt. 2000)

Shan, Xiaoguang; Patentrechte und Know-How im Rechtsverkehr in der Volksrepublik China , Frankfurt am Main 2001. (zit.: Shan, Xiaoguang)

Siemsen, Peter Dirk; Die Entwicklung des gewerblichen Rechtsschutzes in Brasilien Erosion oder Anpassung? GRURInt. 1976 Heft 02. S. 59 ff. (zit.: Siemsen, GRURInt. 1976)

Skaupy, Walther; Know-how-Vereinbarungen und Kartellrecht, GRUR 1964 Heft 10. S. 539 ff. (zit.: Skaupy, GRUR 1964)

Soltysinski, S.; International Exhaustion of Intellectual PRoperty Rights under the TRIPs, the EC Law and the Europe Agreements, GRURInt. 1996, S. 316 ff. (zit.: Soltysinski, GRURInt. 1996)

Sosnitza, Olaf; Gedanken zur Rechtsnatur der ausschließlichen Lizenz, in: Perspektiven des Geistigen Eigentums und Wettbewerbsrechts, Festschrift für Gerhard Schricker zum 70. Geburtstag, München 2005 S. 183 ff. (zit.: Sosnitza)

Staehelin, Alesch; Das TRIPs-Abkommen, Bern 1997. (zit.: Staehelin)

Staudinger, Julius v.; J. von Staudingers Kommentar zum Bürgerlichen Gesetzbuch mit Einführungsgesetz und Nebengesetzen, Buch 2. Recht der Schuldverhältnisse SS 433-487; Leasing (Kaufrecht und Leasingrecht),

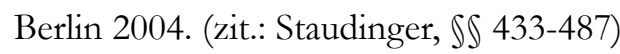

Staudinger, Julius v.; J. von Staudingers Kommentar zum Bürgerlichen Gesetzbuch mit Einführungsgesetz und Nebengesetzen, Buch 2. Recht der Schuldverhältnisse SS 535-562d; Leasing (Kaufrecht und Leasingrecht), Berlin 2003. (zit.: Staudinger, SS 535-562d)

Steinmann, Matthias; Grundzüge des chinesischen Patentrechts, Köln, Berlin, Bonn, München, 1991. (zit.: Steinmann)

Stober, Rolf; Globales Wirtschaftsverwaltungsrecht, Köln 2001

Stober, Rolf; Allgemeines Wirtschaftsverwaltungsrecht, Aufl. 14, Hamburg 2004

Stoffmehl, Thomas; Technologietransfer im europäischen Kartellrecht durch Gruppenfreistellung, Berlin 1997. (zit.: Stoffmehl)

Stoll, Peter-Tobias; Technologietransfer Internationalisierungs- und Nationalisierungstendenzen, Berlin 1994. (zit.: Stoll)

Stoll, Peter-Tobias; Das internationale Recht im Nord-Süd-Verhältnis. Der Technologietransfer, Heidelberg 2005. (zit.: Stoll, 2005)

Stoll, Peter-Tobias; Invisible Hand or Medicines Required? Human Rights in Trade - Inherent or Externalities? Raj Kumar, C./Srivastava D.K. Human Rights and Development: Law, Policy and Governance, Hong Kong 2006. (zit.: Stoll, 2006)

Straus, Joseph; Bedeutung des TRIPS für das Patentrecht, GRURInt. 1996 Heft 03. S. 179 ff. (zit.: Straus, GRURInt. 1996)

Ströbele, Paul/Hacker, Franz; Markengesetz, Kommentar, Aufl. 7, Köln, 2003. 
(zit.: Ströbele/Hacker)

Stumpf, Herbert/Groß Michael; Der Lizenzvertrag, Aufl. 8, Frankfurt am Main 2005. (zit.: Stumpf/Groß)

Stumpf, Herbert; Der Know-How-Vertrag, Aufl.3, Heidelberg 1977. (zit.: Stumpf, Der Know-How-Vertrag)

Stumpf, Herbert; Interessenlage und Interessenkollision beim Technologietransfer: die Rolle des Patentwesens, GRURInt. 1977 Heft 12. S. 441 ff. (zit.: Stumpf, GRURInt. 1977)

Stumpf, Herbert; The Know-How Contract in Germany, Japan and The United States, Frankfurt am Main 1984. (zit.: Stumpf)

Täger, Uwe Christian; Zum Patent- und Lizenzverhalten der deutschen Investitionsgüterindustrie, GRURInt. 1982 Heft 10. S. 604 ff. (zit.: Täger, GRURInt. 1982)

Täger, Uwe Christian; Der Technologietransfer in der Bundesrepublik Deutschland, Berlin 1984. (zit.: Täger, Der Technologietransfer in der BRD)

Tetz, Stefanie; Zur Neuregelung des Patentrechts in der Volksrepublik China, RIW 1993 Heft 6, S. 474 ff. (zit.: Tetz, RIW 1993)

Tetzner, Heinrich; Das materielle Patentrecht der Bundesrepublik Deutschland, Darmstadt 1972. (zit.: Tetzner)

Tetzner, Volkmar; Leitfaden des deutschen Patentrechts, Bern 1970. (zit.: Tetzner, Leitfaden)

Thaler, Paul; Die Durchsetzung der Immaterialgüterrechte in der Schweiz und in der VR China, Zürich 2000. (zit.: Thaler)

Tietje, Christian; Welthandelsorganisation, München 2000. (zit.: Tietje)

Timberg, Sigmund; The Impact on the Transnational Corporation of the International Code of Conduct on the Transfer of Technology, IIC Vol. 12 No. 2/1981. S. 133 ff. (zit.: Timberg, IIC 1981)

Troller, Alois; Internationale Lizenzverträge, GRURAusl. 1952 Heft 03. S. 108 ff. (zit.: Troller, GRURAusl. 1952)

Troller, Alois; Das technische Geheimnis im System des Immaterialgüterrechts, GRURAusl. 1958 Heft 8-9. S. 385 ff. (zit.: Troller, GRURAusl. 1958)

Troller, Alois; Der Schutz des Know-how im schweizerischen Recht, in: Recueil des travaux, Basel 1970, S. 213 ff. (zit.: Troller, Schutz)

Troller, Alois; Immaterialgüterrecht, Band I, Aufl. 3, Basel und Frankfurt am Main, 1983. (zit.: Troller, Immaterialgüterrecht I)

Troller, Alois; Immaterialgüterrecht, Band II, Aufl. 3, Basel und Frankfurt am Main, 1985. (zit.: Troller, Immaterialgüterrecht II)

Übersicht über den Stand der internationalen Verträge auf dem Gebiet des gewerblichen Rechtsschutzes am 1. Januar 2004, GRURInt. 2004 Heft 5. S. $398 \mathrm{ff}$.

Ullrich, Hanns; Technologieschutz nach TRIPS: Prinzipien und Probleme, GRURInt. 1995 Heft 8-9. S. 623 ff. (zit.: Ullrich, GRURInt. 1995)

Ulmer-Eilfort, Constanze/Schmoll, Andrea; Technologietransfer, Lizenzverträge 
für Patente und Know-how, München 2006 (zit.: Ulmer-Eilfort/Schmoll)

United Nations, Joint Ventures as a Form of International Economic Cooperation, New York 1989 (zit.: UN, Joint Ventures)

Vedaraman, S.; The New Indian Patents Law, IIC 1972 Heft 01. S. 39 ff. (zit.: Vedaraman, IIC 1972)

Verma, S. K.; TRIPs - Development and Transfer of Technology, IIC 1996 Heft 03. S. 331 ff. (zit.: Verma, IIC 1996)

Völp, Fromut; Weitergeltung der Lizenz bei Veräußerung des Schutzrechts, GRUR 1983 Heft 02. S. 45 ff. (zit.: Völp, GRUR 1983)

Wadle, Elmar; Geistiges Eigentum, Band I/II, Weinheim 1996. (zit.: Wadle, Geistiges Eigentum I/II)

Wandtke, Artur-Axel/Bullinger, Winfried; Praxiskommentar zum Urheberrecht, Aufl. 2, München 2006. (zit.: Wandtke/Bullinger)

Wang, Hanbin; Minfa Tongze Shi Tiaozheng Shehuizhuyi Shangpin Jingji Guanxi di Jiben Falü (Die AGZR als grundlegendes Gesetz zur Regulierung der Beziehungen in der sozialistischen Warenwirtschaft), FZRB vom 6. 2. 1997

Wang, Jing-An; Der Lizenzvertrag im deutschen und chinesischen Recht, Konstanz 1989. (zit.: Wang, Jing-An)

Weggel, Oskar; Das Außenhandelsrecht der VR China, Baden-Baden 1976.

Weinmann, Conrad; Die Rechtsnatur der Lizenz, Bern 1996. (zit.: Weinmann)

Weiß, Wolfgang/Herrmann, Christoph; Welthandelsrecht, München 2003

Widmer, Beat; Vermögensrechtliche Ansprüche des Inhabers und des Lizenznehmers bei der Verletzung von Immaterialgüterrechten, Basel 1985. (zit.: Widmer)

Wu, Heng; Wirtschaftliche und technologische Entwicklung und die Errichtung eines Patentsystems in der Volksrepublik China, GRURInt. 1983 Heft 08. S. 623 ff. (zit.: Wu, GRURInt. 1983)

Yin, Xintian; The Newly Revised Chinese Patent Act - A Brief Introduction, IIC Vol. 24 No. 2/1993. S. 189 ff. (zit.: Yin, IIC 1993)

Yu, Xiang; Parallelimporte im Recht des geistigen Eigentums Chinas und dessen Entwicklungstendenzen, GRURInt. 2000 Heft 7. S. 619 ff. (zit.: Yu, GRURInt. 2000)

Zhang, Qiang(Hrsg.); Guoji Jishu Zhuanrang Jiufen yu Yufang Anli Fenxi(Fälle zur Streitbeilegung und Streitvorkehrung beim internationalen Technologietransfer), Aufl. 2, Shanxi 1998

Zheng, Chengsi; Zhishi Chanquan Fa(Immaterialgüterrecht), Beijing 1997. (zit.: Zheng, Chengsi)

\section{Gesetzestext}


Patentgesetz der Volksrepublik China, GRURInt. 2001, S. 541 ff.

Durchführungsbestimmungen zum Patentgesetz der Volksrepublik China, GRURInt. 2002, S. 243 ff.

Markengesetz der Volksrepublik China, GRURInt. 2002, S. 489 ff.

Durchführungsverordnung zum Markengesetz, GRURInt. 2003, S. 223 ff.

Urheberrechtsgesetz der Volksrepublik China, GRURInt. 2002, S. 23 ff.

Durchführungsverordnung zum Urheberrechtsgesetz der Volksrepublik China, GRURInt. 2003, S. 1008 ff.

Verordnung über den Schutz von Computersoftware, GRURInt. 2003, S. 47 ff.

Gesetz der VR China gegen den unlauteren Wettbewerb, GRURInt. 1994, S. 1001 ff. 


\section{Lebenslauf}

Zhen Wang, geboren 1979 in Wuhan, China, begann 1997 das Studium der Rechtswissenschaften an der Zhongnan Universität für Wirtschafts- Politik- und Rechtswissenschaft, wo er 2001 mit Bachelor absolvierte. 2004 absolvierte er das Studium der Rechtswissenschaften mit dem Abschluss Magister iur. an der Georg-August-Universität Göttingen. 